\title{
Study of Rare B Meson Decays Related to the CKM Angle Beta at BaBar
}

\author{
Keith Ulmer
}

SLAC-R-870

\author{
Prepared for the Department of Energy \\ under contract number DE-AC02-76SF00515
}

Printed in the United States of America. Available from the National Technical Information Service, U.S. Department of Commerce, 5285 Port Royal Road, Springfield, VA 22161. 
This document, and the material and data contained therein, was developed under sponsorship of the United States Government. Neither the United States nor the Department of Energy, nor the Leland Stanford Junior University, nor their employees, nor their respective contractors, subcontractors, or their employees, makes an warranty, express or implied, or assumes any liability of responsibility for accuracy, completeness or usefulness of any information, apparatus, product or process disclosed, or represents that its use will not infringe privately owned rights. Mention of any product, its manufacturer, or suppliers shall not, nor is it intended to, imply approval, disapproval, or fitness of any particular use. A royalty-free, nonexclusive right to use and disseminate same of whatsoever, is expressly reserved to the United States and the University. 


\title{
Study of Rare $B$ Meson Decays Related to the CKM Angle $\beta$ at BABAR \\ by
}

\author{
Keith Ulmer \\ B.A., Amherst College, 2001

\begin{abstract}
A thesis submitted to the
Faculty of the Graduate School of the

University of Colorado in partial fulfillment

of the requirements for the degree of

Doctor of Philosophy

Department of Phyiscs
\end{abstract} \\ 2007
}

SLAC-R-870

Work supported [in part] by U.S. Department of Energy contract DE-AC02-76SF00515 
This thesis entitled:

Study of Rare $B$ Meson Decays Related to the CKM Angle $\beta$ at BABAR written by Keith Ulmer

has been approved for the Department of Phyiscs

Prof. James G. Smith

Prof. William T. Ford

Date

The final copy of this thesis has been examined by the signatories, and we find that both the content and the form meet acceptable presentation standards of scholarly work in the above mentioned discipline. 
Ulmer, Keith (Ph.D., Physics)

Study of Rare $B$ Meson Decays Related to the CKM Angle $\beta$ at BABAR

Thesis directed by Prof. James G. Smith

This study reports measurements of the branching fractions of $B$ meson decays to $\eta^{\prime} K^{+}, \eta^{\prime} K^{0}, \omega \pi^{+}, \omega K^{+}$, and $\omega K^{0}$. Charge asymmetries are measured for the charged modes and the time-dependent $C P$-violation parameters $S$ and $C$ are measured for the neutral modes. The results are based on a data sample of $347 \mathrm{fb}^{-1}$ containing 383 million $B \bar{B}$ pairs recorded by the BABAR detector at the PEP-II asymmetric-energy $e^{+} e^{-}$storage ring located at the Stanford Linear Accelerator Center.

Statistically significant signals are observed for all channels with the following results: $\mathcal{B}\left(B^{+} \rightarrow \eta^{\prime} K^{+}\right)=(70.0 \pm 1.5 \pm 2.8) \times 10^{-6}, \mathcal{B}\left(B^{0} \rightarrow \eta^{\prime} K^{0}\right)=(66.6 \pm 2.6 \pm 2.8) \times$ $10^{-6}, \mathcal{B}\left(B^{+} \rightarrow \omega \pi^{+}\right)=(6.7 \pm 0.5 \pm 0.4) \times 10^{-6}, \mathcal{B}\left(B^{+} \rightarrow \omega K^{+}\right)=(6.3 \pm 0.5 \pm 0.3) \times 10^{-6}$ and $\mathcal{B}\left(B^{0} \rightarrow \omega K^{0}\right)=(5.6 \pm 0.8 \pm 0.3) \times 10^{-6}$, where the first uncertainty is statistical and the second is systematic. We measure $\mathcal{A}_{c h}\left(\eta^{\prime} K^{+}\right)=+0.010 \pm 0.022 \pm 0.006, \mathcal{A}_{c h}\left(\omega \pi^{+}\right)=$ $-0.02 \pm 0.08 \pm 0.01, \mathcal{A}_{c h}\left(\omega K^{+}\right)=-0.01 \pm 0.07 \pm 0.01, S_{\eta^{\prime} K_{S}^{0}}=0.56 \pm 0.12 \pm 0.02, C_{\eta^{\prime} K_{S}^{0}}$ $=-0.24 \pm 0.08 \pm 0.03, S_{\omega K_{S}^{0}}=0.62_{-0.29}^{+0.25} \pm 0.02$, and $C_{\omega K_{S}^{0}}=-0.39_{-0.24}^{+0.25} \pm 0.03$. The result in $S_{\eta^{\prime} K_{S}^{0}}$ contributes to the published measurement from BABAR, which differs from zero by 5.5 standard deviations and is the first observation of mixing-induced $C P$-violation in a charmless $B$ decay. 


\section{Acknowledgements}

Many, many people have been enormously helpful and supportive over the course of this work. I'd like to begin with Jim who has been a great adviser and mentor to me. His constant encouragement has helped lead me along at every step of the way. I appreciate his dedication to the physics and also to his students. Next, I'd like to thank Bill whose leadership style is the perfect complement to Jim. Together they have created an outstanding group to work with. I don't think I would have learned nearly as much without Fred always being around to discuss anything at all and to answer my "stupid questions." Thanks, too, to Jim Hirschauer for being a great office mate, sounding board and friend. Many others in the group over the years have helped me in a variety of ways, too, including Paul, Arik, Lei, Corry, Ian...

None of this would would have been possible without a ton of effort from the entire BABAR collaboration and PEP-II staff. There are too many people to list, but HEP experiment is truly collaborative, and I'm grateful for all the work that went in to making these results possible. In particular, the Q2B AWG has contributed greatly to this analysis, especially our collaborators Alfio and Fernando.

Thanks to the other HEP grad students for support and conversation: Mike, Bob, Rune... Thanks to the grad students I took classes with for being good study partners and becoming good friends: Juan, Marty, Kirk, Chris, Zach, Amanda, Ashley, John... Thanks to the CU professors serving on my defense and examination committees: Anna Hasenfratz, Ed Kinney, Tom DeGrand and Andrew Hamilton. 
Finally, thanks to all my friends and family for unending support through the years, especially: My mom and Jeff, my brother Alex, my dad and Connie, and my loving wife, Allison, who has put up with quite a bit and always continued to encourage me. 


\section{Contents}

\section{Chapter}

1 Theory 1

1.1 The Standard Model . . . . . . . . . . . . . . . . . . . . . . 1

1.2 Quark Mixing . . . . . . . . . . . . . . . . . . 4

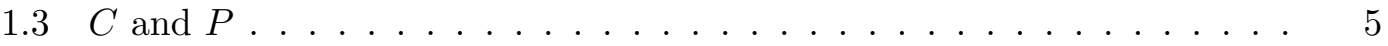

$1.4 C P$ Violation in $B^{0}$ Mesons . . . . . . . . . . . . . . . . 7

1.5 Time Evolution of $B \bar{B}$ Pairs from $\Upsilon(4 S) \ldots \ldots \ldots . \ldots$

1.6 Types of $C P$ Violation . . . . . . . . . . . . . . . . . . 10

1.6.1 CP Violation in Decay . . . . . . . . . . . . . . . 10

1.6.2 $C P$ Violation in Mixing . . . . . . . . . . . . . . . 12

1.6.3 CP Violation in the Interference Between Mixing and Decay . . 12

1.7 CKM Contribution from $B \bar{B}$ Mixing . . . . . . . . . . . . . . 13

$1.8 C P$ Violation in $B^{0} \rightarrow \eta^{\prime} K_{S}^{0}$ and $\sin 2 \beta \ldots \ldots \ldots \ldots \ldots$

1.9 Branching Fractions in $B \rightarrow \eta^{\prime} K$ Decays . . . . . . . . . . . . . . 17

$1.10 C P$ Violation in $B^{0} \rightarrow \omega K_{S}^{0}$ and $\sin 2 \beta \ldots \ldots \ldots . \ldots . \ldots . \ldots$

1.11 Branching Fractions in $B \rightarrow \omega K$ and $B \rightarrow \omega \pi$ Decays . . . . . . . . 19

1.12 Previous Results . . . . . . . . . . . . . . . . . . 20

2 The BABAR Experiment 22

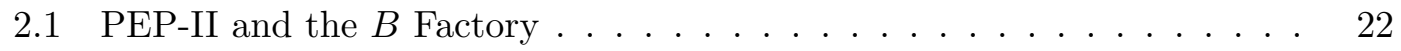


2.2 The BABAR Detector . . . . . . . . . . . . . . . 27

2.2 .1 Silicon Vertex Tracker . . . . . . . . . . . . . . . . 27

2.2 .2 Drift Chamber . . . . . . . . . . . . . . . . . . 29

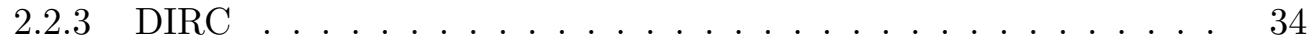

2.2.4 Electro-Magnetic Calorimeter . . . . . . . . . . . . 36

2.2 .5 Instrumented Flux Return . . . . . . . . . . . . . . . . . 38

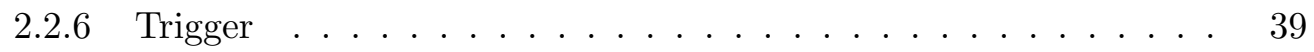

3 Analysis Technique 41

3.1 Analysis Overview . . . . . . . . . . . . . . . . . . . 41

3.2 Data, Monte Carlo Samples and Processing . . . . . . . . . . . . . . . 42

3.3 Reconstruction and Event Selection . . . . . . . . . . . . . . . 42

3.3.1 Candidate Reconstruction . . . . . . . . . . . . . . . 42

3.3 .2 Kinematic Variables . . . . . . . . . . . . . . . . . . 44

3.3.3 Event Shape Variables . . . . . . . . . . . . . . . 45

3.3.4 Final Selection Cuts . . . . . . . . . . . . . . . . 47

3.4 Maximum Likelihood Fit . . . . . . . . . . . . . . . . . . . . . . . 49

3.4 .1 Fit Variables . . . . . . . . . . . . . . . . . 49

3.4.2 PDFs and the Likelihood ................ 50

3.5 Joint $K-\pi$ Fit $\ldots \ldots \ldots \ldots \ldots \ldots \ldots \ldots \ldots \ldots$

3.5.1 The $B$ Vertices and $\Delta z \ldots \ldots \ldots \ldots \ldots$

3.5.2 $\Delta z \rightarrow \Delta t$ Conversion . . . . . . . . . . . . . 53

3.5 .3 BReco Sample . . . . . . . . . . . . . . . . . 54

3.5 .4 Flavor Tagging . . . . . . . . . . . . . . . . . 54

3.6 Probability Density Functions . . . . . . . . . . . . . 56

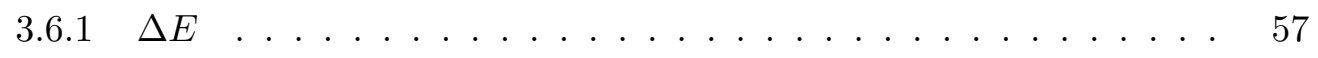

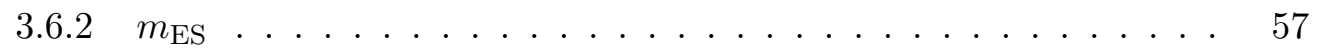


3.6.3 Resonance Masses . . . . . . . . . . . . . . . . . 58

3.6.4 Fisher Discriminant . . . . . . . . . . . . . . . 60

3.6.5 Resonance Helicity . . . . . . . . . . . . . . . . . . . . . . 60

3.6.6 Correlations Among Discriminating Variables . . . . . . . . . . . 61

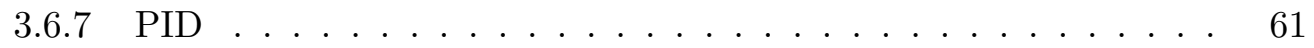

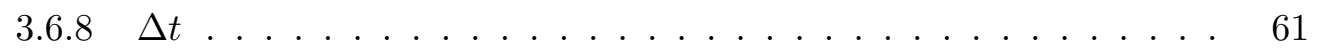

3.6.9 Floating Background Parameters . . . . . . . . . . 63

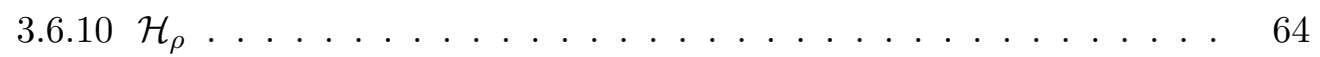

3.7 Efficiency, Corrections and Production Rate . . . . . . . . . . . . . . 64

3.8 Two-track Backgrounds . . . . . . . . . . . . . . . . 66

$3.9 B \bar{B}$ Backgrounds . . . . . . . . . . . . . . . . 66

3.10 Fit Validation . . . . . . . . . . . . . . . . . . 69

3.10 .1 Validation of Yield Fits . . . . . . . . . . . . . 69

$3.10 .2 \mathcal{A}_{c h}$ Bias Effect . . . . . . . . . . . . . 72

3.10 .3 Validation of $C P$ Fits $\ldots \ldots \ldots 73$

4 Results $\quad 78$

4.1 Branching Fractions and Charge Asymmetry Results . . . . . . . . . 78

4.1.1 Branching Fraction Fit Results . . . . . . . . . . . 78

4.1 .2 Projection Plots . . . . . . . . . . . . . 81

4.1 .3 sPlots ............................. 85

4.1.4 Likelihood Ratio Plots . . . . . . . . . . . . . . . . . . 93

4.2 Time-dependent Results . . . . . . . . . . . . . . . . . . . . . . . 98

$4.2 .1 \quad C P$ Fits $\ldots \ldots \ldots \ldots \ldots$

4.2.2 Discussion of $B \bar{B}$ Background . . . . . . . . . . . . . . 98

4.2.3 Pull Widths and Underestimated Errors . . . . . . . . . . . . . 99

4.2 .4 Projection Plots . . . . . . . . . . . . . . . 101 
4.2 .5 sPlots ............................ 101

4.2 .6 Likelihood Plots . . . . . . . . . . . . . . 106

4.2 .7 Combining NLL Plots . . . . . . . . . . . . . . 106

4.3 Systematic Uncertainties for the Branching Fraction Fits . . . . . . . . . 112

4.3.1 Branching Fraction Systematic Uncertainties . . . . . . . . . . . 112

4.3.2 Charge Asymmetry Systematic Uncertainties . . . . . . . . . . 117

4.4 Systematic Uncertainties for Time-dependent Asymmetry Fits . . . . 118

5 Discussion

\section{Appendix}

$\begin{array}{lr}\text { A Particle Lists } & 128\end{array}$

A.1 GoodTracksVeryLoose . . . . . . . . . . . . . . . . . 128

A.2 GoodTracksLoose . . . . . . . . . . . . . . . . . 128

A.3 ChargedTracks . . . . . . . . . . . . . . . . . . . . . . 128

$\begin{array}{lr}\text { B Fits to BReco Data } & 129\end{array}$

$\begin{array}{lr}\text { C PDF Libraries } & 130\end{array}$

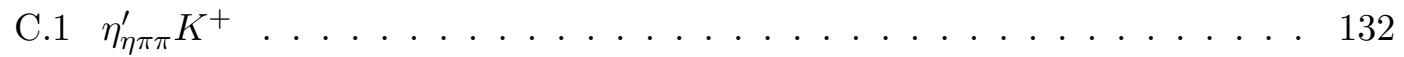

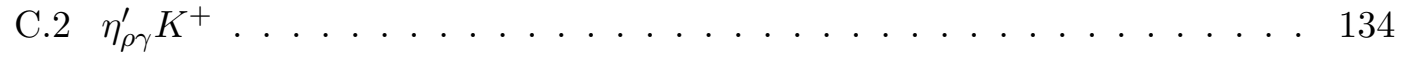

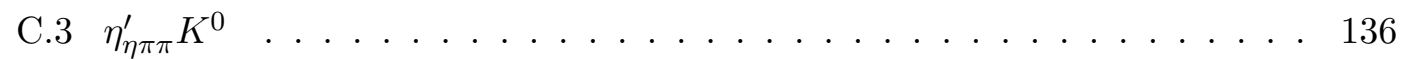

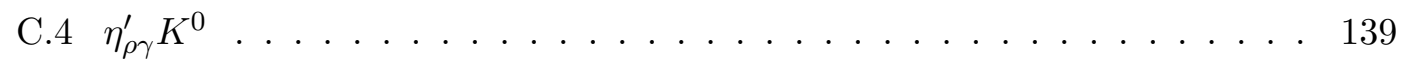

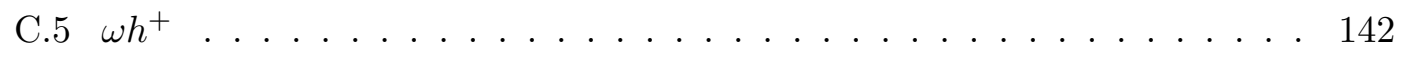

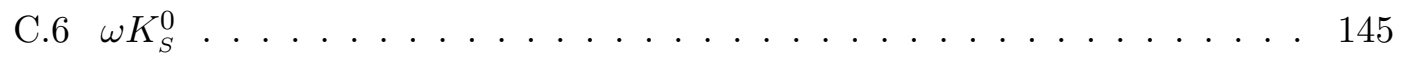

$\begin{array}{lr}\text { D Fit Variable Correlations } & 149\end{array}$

$\begin{array}{ll}\text { E Pure Toy Pulls } & 152\end{array}$ 
F Combining Results with Log Likelihood Curves 157

F.1 Log Likelihood Curve Basics . . . . . . . . . . . . . . . . . . . 158

F.2 Statistical Significance with Systematics . . . . . . . . . . . . 158

F.3 Combining Branching Fractions and Asymmetries with Systematics . . . 159

F.4 The combine.cc Script . . . . . . . . . . . . . . . . . 159

$\begin{array}{ll}\text { G Overlap Bug Effect } & 161\end{array}$

Bibliography 


\section{Tables}

Table

1.1 Standard Model particles. . . . . . . . . . . . . . . . . . . 3

1.2 Summary of previous results. . . . . . . . . . . . . . . 21

2.1 PEP-II beam parameters. . . . . . . . . . . . . . . 23

2.2 Cross sections for $e^{+} e^{-}$collisions at $10.58 \mathrm{GeV} \ldots \ldots \ldots$. . . . . 40

3.1 Tagging category fractions and mistag rates. . . . . . . . . . . . 56

3.2 Data/MC corrections in PDFs. . . . . . . . . . . . . . 60

$3.3 B \bar{B}$ background in $B^{+} \rightarrow \eta_{\rho \gamma}^{\prime} K^{+} \ldots \ldots \ldots \ldots \ldots \ldots \ldots$

$3.4 B \bar{B}$ background in $B^{0} \rightarrow \eta_{\rho \gamma}^{\prime} K^{0} \ldots \ldots \ldots \ldots$. . . . . . . 68

$3.5 B \bar{B}$ background in $B^{+} \rightarrow \omega h^{+} \ldots \ldots \ldots \ldots$. . . . . . . . 70

3.6 $B \bar{B}$ background in $B^{0} \rightarrow \omega K^{0}$. . . . . . . . . . . . . . . 71

3.7 Embedded toy $\mathrm{MC}$ results for yield fits. . . . . . . . . . . . . . . 72

3.8 Summary of results from embedded toy MC studies in $\omega h^{+}$. We use 500 toy experiments, embedding signal MC events with the $\mathcal{A}_{c h}$ as indicated in the table. All numbers are in events. . . . . . . . . . . . 73

3.9 Embedded toy results for $\eta^{\prime} K_{S}^{0} \Delta t$ fits. . . . . . . . . . . 74

3.10 Embedded toy results for $\omega K_{S}^{0} \Delta t$ fits . . . . . . . . . . . . 76

3.11 Toy experiment comparison of BReco and signal MC parameters. . . . . 77

4.1 Branching fraction and charge asymmetry results for the $B \rightarrow \eta^{\prime} K$ modes. 79 
4.2 Branching fraction and charge asymmetry results for the $\omega$ modes. . . 79

4.3 Results for the $B^{0} \rightarrow \eta^{\prime} K_{S}^{0}$ and $B^{0} \rightarrow \omega K_{S}^{0} \Delta t$ fits. . . . . . . . . 98

4.4 Embedded toy results for $\eta_{\rho \gamma}^{\prime} K^{0} \Delta t$ fits. . . . . . . . . . . . . 99

4.5 Embedded toy results for $\omega K_{S}^{0} \Delta t$ fits. . . . . . . . . . . 99

4.6 Combining method comparison for $\eta^{\prime} K_{S}^{0} \Delta t$ fit results. . . . . . . . . . 111

4.7 Systematic errors for $\eta^{\prime} K$ yield fits. . . . . . . . . . . . . . . . . 113

4.8 Systematic errors for $\omega$ yield fits. . . . . . . . . . . . . . . 114

4.9 Estimates of systematic errors for $\Delta t$ fits. . . . . . . . . . . . 120

4.10 Systematic error fit parameter variation results. . . . . . . . . . . . 121

5.1 Summary of results. . . . . . . . . . . . . . . . . . . 122

B.1 Results of the fit to BReco events. . . . . . . . . . . . . . . . 129

B.2 Summary of BReco signal resolution function parameters. . . . . . . . 129

C.1 Explanation of variable names used in RooRarFit. . . . . . . . . . . . 131

E.1 Pure toy pulls for $\eta_{\eta \pi \pi}^{\prime} K^{+}$yield fits. . . . . . . . . . . . . . . 152

E.2 Pure toy pulls for $\eta_{\rho \gamma}^{\prime} K^{+}$yield fits. . . . . . . . . . . . . . 152

E.3 Pure toy pulls for $\eta_{\eta \pi \pi}^{\prime} K_{S}^{0}$ yield fits. . . . . . . . . . . . . 153

E.4 Pure toy pulls for $\eta_{\rho \gamma}^{\prime} K_{S}^{0}$ yield fits. . . . . . . . . . . . 153

E.5 Pure toy pulls for $\omega h^{+}$yield fits. . . . . . . . . . . . . . . 154

E.6 Pure toy pulls for $\omega K_{S}^{0}$ yield fits. . . . . . . . . . . . . . . . 154

E.7 Pure toy pulls for $\eta_{\eta \pi \pi}^{\prime} K_{S}^{0} \Delta t$ fits. . . . . . . . . . . . 155

E.8 Pure toy pulls for $\eta_{\rho \gamma}^{\prime} K_{S}^{0} \Delta t$ fits. . . . . . . . . . . 155

E.9 Pure toy pulls for $\omega K_{S}^{0} \Delta t$ fits. . . . . . . . . . . . . 156

G.1 Comparison of efficiencies with and without overlap bug. . . . . . . . . 162 


\section{Figures}

\section{Figure}

1.1 The CKM unitarity triangle. . . . . . . . . . . . . 6

1.2 Box diagram describing $B^{0}-\bar{B}^{0}$ mixing in the SM. . . . . . . . . . . 14

1.3 Tree diagram describing the decay $\bar{B}^{0} \rightarrow J / \psi K_{S}^{0} \ldots \ldots \ldots \ldots$

1.4 Feynman diagrams describing the decay $B^{0} \rightarrow \eta^{\prime} K^{0} \ldots \ldots \ldots \ldots$

1.5 Feynman diagrams describing the decay $B^{0} \rightarrow \omega^{\prime} K^{0} \ldots \ldots$. . . . . . . 19

1.6 Feynman diagrams describing the decays $B^{+} \rightarrow \omega \pi^{+}$and $B^{+} \rightarrow \omega K^{+} . .20$

2.1 Integrated luminosity . . . . . . . . . . . . . . . . 24

2.2 Schematic of the linac, PEP-II and IR-2. . . . . . . . . . . . . 24

2.3 Beam crossing. . . . . . . . . . . . . . 26

2.4 End view of the BABAR detector. . . . . . . . . . . . 28

2.5 Longitudinal view of the BABAR detector. . . . . . . . . . . 28

2.6 Longitudinal view of the SVT. . . . . . . . . . . . . . . . . . 29

2.7 Transverse view of the SVT. . . . . . . . . . . . . . . 30

2.8 DCH drift cell configuration. . . . . . . . . . . . . . . . 32

2.9 DCH drift cell. . . . . . . . . . . . . . . . . . . . . . 33

2.10 Schematic view of the DIRC silica bar and imaging PMTs. . . . . . . 35

2.11 PID performance plots. . . . . . . . . . . . . . . 37

2.12 Vertical-slice view of the DIRC . . . . . . . . . . . . . . . 37 
2.13 Longitudinal cross-section of the top half of the EMC. . . . . . . . . 38

2.14 Barrel (left) and endcap (right) sections of the IFR. . . . . . . . . . 39

$3.1\left|\cos \theta_{\mathrm{T}}\right|$ distribution for signal and background. . . . . . . . . . . . 46

3.2 Fit to $m_{\mathrm{ES}} q \bar{q}$ distribution for $B^{+} \rightarrow \eta_{\rho \gamma}^{\prime} K^{+}$with endpoint floating. . . 58

3.3 DIRC pull distribution for signal and background. . . . . . . . . . . . 62

3.4 Pure toy pulls for $S$ and $C$ in $B^{0} \rightarrow \omega K^{0} \ldots \ldots \ldots . \ldots . \ldots 75$

$4.1-2 \ln \mathcal{L}$ branching fraction combining curves for $\eta^{\prime} K$ modes. . . . . . . . 80

$4.2-2 \ln \mathcal{L} \mathcal{A}_{c h}$ combining curves for $\eta^{\prime} K^{+} \ldots \ldots \ldots \ldots \ldots$

4.3 Projection plots for $\eta^{\prime} K$ yield fits. . . . . . . . . . . . . 81

4.4 Projection plots for $\omega \pi^{+}$yield fit. . . . . . . . . . . . . 82

4.5 Projection plots for $\omega K^{+}$yield fit. . . . . . . . . . . . 83

4.6 Projection plots for $\omega K_{S}^{0}$ yield fit. . . . . . . . . . . . 84

4.7 sPlots for $\eta_{\eta \pi \pi}^{\prime} K^{+}$yield fit. . . . . . . . . . . 86

4.8 sPlots for $\eta_{\rho \gamma}^{\prime} K^{+}$yield fit. . . . . . . . . . . . 87

4.9 sPlots for $\eta_{\eta \pi \pi}^{\prime} K_{S}^{0}$ yield fit. . . . . . . . . . . 88

4.10 sPlots for $\eta_{\rho \gamma}^{\prime} K_{S}^{0}$ yield fit. . . . . . . . . . . . . 89

4.11 sPlots for $\omega h^{+}$yield fit. . . . . . . . . . . . . . . 90

4.12 sPlots for $\omega h^{+}$yield fit. . . . . . . . . . . . . . 91

4.13 sPlots for $\omega K_{S}^{0}$ yield fit. . . . . . . . . . . . . . 92

4.14 Likelihood ratio for $\eta^{\prime} K^{+}$yield fits. . . . . . . . . . . . . 94

4.15 Likelihood ratio for $\eta^{\prime} K_{S}^{0}$ yield fits. . . . . . . . . . . . . 95

4.16 Likelihood ratio for $\omega h^{+}$yield fit. . . . . . . . . . . . 96

4.17 Likelihood ratio for $\omega K_{S}^{0}$ yield fit. . . . . . . . . . . . . 97

4.18 Embedded toy error distributions for $\omega K_{S}^{0} \Delta t$ fit. . . . . . . . . . . 100

4.19 Embedded toy pull distributions for $S$ and $C$ for $\eta^{\prime} K_{S}^{0} \ldots \ldots \ldots 1$

4.20 Projection plots for $\eta^{\prime} K_{S}^{0} \Delta t$ fits. . . . . . . . . . . . . . . 102 
4.21 Projection plots of $\Delta t$ for combined $\eta^{\prime} K_{S}^{0}$ submodes. . . . . . . . . . 103

4.22 Projection plots for $\omega K_{S}^{0} \Delta t$ fit. . . . . . . . . . . . . . 104

4.23 Projection plots of $\Delta t$ for $\omega K_{S}^{0} \ldots \ldots \ldots \ldots \ldots \ldots$

4.24 sPlots for $\eta_{\eta \pi \pi}^{\prime} K_{S}^{0} \Delta t$ fit. . . . . . . . . . . . . . 107

4.25 sPlots for $\eta_{\rho \gamma}^{\prime} K_{S}^{0} \Delta t$ fit. . . . . . . . . . . . . . 108

4.26 sPlots for $\omega K_{S}^{0} \Delta t$ fit. . . . . . . . . . . . . . 109

4.27 Likelihood ratio for $\eta^{\prime} K_{S}^{0} \Delta t$ fits. . . . . . . . . . . . . . . 110

4.28 Likelihood ratio for $\omega K_{S}^{0} \Delta t$ fit. . . . . . . . . . . . . . . . 110

$4.29-2 \ln \mathcal{L} S$ and $C$ combining curves for $\eta^{\prime} K_{S}^{0}$ modes. . . . . . . . . . 111

5.1 Experimental results for $S$ for all measured decay channels. . . . . . . . 125

5.2 Experimental results for $C$ for all measured decay channels. . . . . . . . 127

C.1 PDF plots for $\eta_{\eta \pi \pi}^{\prime} K^{+} \ldots \ldots \ldots \ldots \ldots \ldots \ldots \ldots \ldots$

C.2 PDF plots for $\eta_{\rho \gamma}^{\prime} K^{+} \ldots \ldots \ldots \ldots \ldots \ldots \ldots \ldots \ldots$

C.3 PDF plots for $\eta_{\eta \pi \pi}^{\prime} K_{S}^{0} \ldots \ldots \ldots \ldots \ldots \ldots \ldots \ldots$

C.4 PDF plots for $\eta_{\rho \gamma}^{\prime} K_{S}^{0}$. . . . . . . . . . . . . . . . 141

C.5 PDF plots for $w h^{+} \ldots \ldots \ldots \ldots \ldots \ldots \ldots$. . . . . . . . . . . . . . . . .

C.6 PDF plots for $\omega K_{S}^{0} . \ldots \ldots \ldots \ldots \ldots \ldots$

D.1 Profile plots from data with signal removed for $\eta_{\rho \gamma}^{\prime} K^{+} \ldots \ldots \ldots$. . . 150

D.2 Profile plots from data with signal removed for $\eta_{\rho \gamma}^{\prime} K^{+}$detailed view. . . 151 


\section{Chapter 1}

\section{Theory}

\subsection{The Standard Model}

The Standard Model of particle physics (SM) provides the framework for our current understanding of the fundamental interactions of nature. It describes a world made up of three generations of quark pairs and three matching generations of lepton pairs with the fundamental interactions mediated by the exchange of vector bosons.

The SM is a quantum field theory where the Lagrangian is invariant under a set of gauge transformations associated with the vector bosons, which are also known as the gauge bosons. Mathematically, these gauge transformations can be described using unitary groups.

The photon is the gauge boson that mediates the electromagnetic (EM) force as described by Quantum Electrodynamics (QED). It couples to particles with electromagnetic charge to mediate the interaction. The fields describing particles interacting electromagnetically, such as the electron, can be rotated by a constant complex phase and are found to leave the Lagrangian unchanged when a concurrent gauge transformation is applied to the gauge field. This rotation leaves the Lagrangian invariant under the operation of an infinite family of phase transformations described by the unitary Abelian group, U(1). Through Noether's theorem, this invariance leads to the existence of a conserved current, in this case identified as the electric charge, Q. This pattern of an invariance under a gauge group and subsequent conservation law for a fundamental 
quantity is repeated for the weak and strong interactions as well.

Weak interactions are mediated by the massive $W^{ \pm}$and $Z^{0}$ bosons. The EM and weak interactions were shown to be unified in an $S U(2) \times U(1)$ gauge group structure in 1967 [1]. The weak structure and unification with QED depends on the spontaneous breaking of the gauge symmetry to produce the three massive and one massless gauge bosons. It is possible to introduce a gauge-invariant complex isospin doublet with a spontaneously broken vacuum expectation value such that the electromagnetic $\mathrm{U}(1)$ $\left(U(1)_{e m}\right)$ symmetry remains unbroken with a massless photon, but the weak mediators, the $W^{ \pm}$and the $Z^{0}$, acquire mass. To achieve this, $U(1)_{e m}$ is structured as a combination of "weak isospin" $\mathrm{SU}(2)$ and "weak hypercharge" $\mathrm{U}(1)$, both of which are broken individually, but leave $U(1)_{e m}$ unbroken. In this case, the gauge structure consists of doublets invariant under weak $\mathrm{SU}(2)$ rotations. The lepton doublets consist of a neutrino and a negatively-charged massive lepton, $e^{-}, \mu^{-}$, or $\tau^{-}$. The quark doublets consist of an up-type quark (u, c, or t) and a down-type quark (d, s, or b). Only the left-handed fermion doublets are found to couple to the weak currents. Transformation properties of these fermion doublets are discussed further in Sec. 1.2.

The same spontaneously broken iso-doublet also provides the mechanism for the fermions to acquire mass in a gauge-invariant way. A single neutral scalar Higgs field arises from the choice of a non-zero vacuum expectation value for the iso-doublet. This is the Higgs boson, which has yet to be observed in nature, lingering as the only unobserved particle in the SM.

The strong interaction is described by Quantum Chromodynamics (QCD) where interactions between particles with non-zero "color charge" are mediated by gauge vector bosons known as gluons. Leptons are said to be "colorless" and do not participate in the strong interaction. The color charge comes in three varieties, commonly referred to as "red," "blue," and "green," and their antiparticle conjugate. Each quark contains one color charge, and the symmetry between them is exact forming a triplet structure. The 
strength of the strong interaction grows with the distance separating two color-charged objects. At large enough distances the energy required to pull two quarks apart becomes sufficient to generate a quark-anti-quark pair from the vacuum, and therefore free quarks or gluons have never been observed. Free particles exist only in "colorless" combinations of three-quark baryons $(r g b$, or $\bar{r} \bar{g} \bar{b})$ or two-quark mesons $(r \bar{r}, g \bar{g}$, or $b \bar{b})$.

In total, the SM can be described in the unitary group notation as $S U(3)_{C} \times$ $S U(2)_{L} \times U(1)_{Y}$, where the "C" stands for the strong interaction's color charge, the "L" refers to the left-handed fermion doublets that couple to the weak currents, and the "Y" stands for hypercharge. Table 1.1 lists the fundamental constituents of the Standard Model.

Table 1.1: The particles in the Standard Model of particle physics. Antiparticles are not shown, though are included in the SM with the opposite electric charge. Mass information comes from the Particle Data Group [2].

\begin{tabular}{lcccc}
\hline \hline Particle & symbol & spin $(\hbar)$ & electric charge $(\mathrm{e})$ & $\operatorname{mass}(\mathrm{MeV}[3])$ \\
\hline \multicolumn{1}{c}{ Quarks } & $\mathrm{d}$ & $\frac{1}{2}$ & $-\frac{1}{3}$ & $3-7$ \\
down & $\mathrm{u}$ & $\frac{1}{2}$ & $+\frac{2}{3}$ & $1.5-3.0$ \\
up & $\mathrm{s}$ & $\frac{1}{2}$ & $-\frac{1}{3}$ & $70-120$ \\
strange & $\mathrm{c}$ & $\frac{1}{2}$ & $+\frac{2}{3}$ & $(1.25 \pm 0.09) \times 10^{3}$ \\
charm & $\mathrm{b}$ & $\frac{1}{2}$ & $-\frac{1}{3}$ & $(4.20 \pm 0.07) \times 10^{3}$ \\
bottom & $\mathrm{t}$ & $\frac{1}{2}$ & $+\frac{2}{3}$ & $(1.742 \pm 0.033) \times 10^{5}$ \\
top & & & & \\
\hline Leptons & $e^{-}$ & $\frac{1}{2}$ & -1 & 0.511 \\
electron & $\nu_{e}$ & $\frac{1}{2}$ & 0 & $\sim 0$ \\
electron neutrino & $\mu^{-}$ & $\frac{1}{2}$ & -1 & $\sim 0$ \\
muon & $\nu_{\mu}$ & $\frac{1}{2}$ & 0 & $1777.0 \pm 0.3$ \\
muon neutrino & $\tau^{-}$ & $\frac{1}{2}$ & -1 & $\sim 0$ \\
tau & $\nu_{\tau}$ & $\frac{1}{2}$ & 0 & 0 \\
tau neutrino & & & & $80.406 \pm 0.029$ \\
\hline \multicolumn{1}{c}{ Gauge bosons } & $\gamma$ & 1 & 0 & $91.188 \pm 0.002$ \\
photon & $W^{ \pm}$ & 1 & \pm 1 & 0 \\
$W^{ \pm}$ & $Z^{0}$ & 1 & 0 & $>114$ \\
$Z^{0}$ & $\mathrm{~g}$ & 1 & 0 & \\
gluon & $h^{0}$ & 0 & & 0 \\
\hline Higgs boson & & & 0 & \\
\hline \hline
\end{tabular}


The SM does a remarkable job of providing a structure of fundamental constituents (fermions, leptons, and gauge bosons) and interactions capable of describing the observed particles in the universe. The theory, however, is not capable of giving absolute predictions for the size and strength of all the interactions independently. For example, the fermion masses are proportional to the strength of their coupling to the Higgs field, but the masses themselves must be measured by experiment and input into the theory. Similarly the strengths of the coupling constants for each of the gauge groups must also be measured experimentally.

Further, to this point the SM does not include a description of the final fundamental force, gravity, though it is too weak to be relevant on the scale of the interactions discussed here.

\subsection{Quark Mixing}

In the electroweak theory, the left-handed fermions are $\mathrm{SU}(2)$ doublets within each of the three generations of leptons and quarks. The weak quark doublets, however, are not the same as the flavor (or mass) eigenstates that couple to the Higgs field. This allows for mixing of the generations of quarks, described in the SM by the CabibboKobayashi-Maskawa (CKM) mixing matrix [4] shown in Eq. 1.1, where the primed states represent the weak eigenstates and the unprimed states represent the mass eigenstates.

$$
\left(\begin{array}{c}
d^{\prime} \\
s^{\prime} \\
b^{\prime}
\end{array}\right)=\left(\begin{array}{ccc}
V_{u d} & V_{u s} & V_{u b} \\
V_{c d} & V_{c s} & V_{c b} \\
V_{t d} & V_{t s} & V_{t b}
\end{array}\right)\left(\begin{array}{l}
d \\
s \\
b
\end{array}\right)
$$

Quark flavor changing processes only occur in the weak interaction in the SM with the exchange of a $W^{ \pm}$boson. Since no flavor changing neutral currents are allowed, an up-type quark must change into a down-type quark and vice versa. However the mixing matrix allows for an up-type quark of one generation to transition into a down- 
type quark of another generation. In essence, the charged current couples to a doublet structure that is slightly rotated with respect to the pure flavor doublets.

To conserve total probability in these interactions, the CKM matrix is unitary. Any $3 \times 3$ unitary matrix can be characterized by three Euler angles and one non-trivial phase [5]. Here the angles are rotations in flavor space and the non-trivial phase is ultimately responsible for $C P$ violation in the SM [6] as discussed in Sec. 1.3.

The CKM matrix is commonly expressed in the Wolfenstein parameterization [7] in terms of the four real parameters: $\lambda, A, \rho$ and $\eta$. Expanding in terms of $\lambda=$ $\sin \theta_{\text {Cabibbo }}=\left|V_{u s}\right| \approx 0.22$ with the other parameters of $\mathcal{O}(1)$,

$$
V_{C K M}=\left(\begin{array}{ccc}
1-\frac{1}{2} \lambda^{2} & \lambda & A \lambda^{3}(\rho-i \eta) \\
-\lambda & 1-\frac{1}{2} \lambda^{2} & A \lambda^{2} \\
A \lambda^{3}(1-\rho-i \eta) & -A \lambda^{2} & 1
\end{array}\right)+\mathcal{O}\left(\lambda^{4}\right) .
$$

All of the complex phase information is captured in the parameter $\eta$. The unitarity of $V_{C K M}$ can be depicted as a series of six triangles in the complex plane. One such triangle results in sides of comparable length given by the relationship,

$$
V_{u d} V_{u b}^{*}+V_{c d} V_{c b}^{*}+V_{t d} V_{t b}^{*}=0
$$

This triangle, known as the "Unitarity Triangle," can be rescaled and rotated to a base of unit length with an apex of $(\rho, \eta)$ as depicted in Fig. 1.1. The three angles in the figure, $\alpha, \beta$ and $\gamma$, appear in Eq. 1.2 as the complex phase. They can also be written in terms of the CKM matrix elements,

$$
\alpha \equiv \arg \left[-\frac{\mathrm{V}_{\mathrm{td}} \mathrm{V}_{\mathrm{tb}}^{*}}{\mathrm{~V}_{\mathrm{ud}} \mathrm{V}_{\mathrm{ub}}^{*}}\right], \beta \equiv \arg \left[-\frac{\mathrm{V}_{\mathrm{cd}} \mathrm{V}_{\mathrm{cb}}^{*}}{\mathrm{~V}_{\mathrm{td}} \mathrm{V}_{\mathrm{tb}}^{*}}\right] \text {, and } \gamma \equiv \arg \left[-\frac{\mathrm{V}_{\mathrm{ud}} \mathrm{V}_{\mathrm{ub}}^{*}}{\mathrm{~V}_{\mathrm{cd}} \mathrm{V}_{\mathrm{cb}}^{*}}\right]
$$

\section{3 $C$ and $P$}

Charge conjugation $(C)$ is defined as the operation of switching all particles involved in a process with their corresponding anti-particles. For a physical theory, this 
Figure 1.1: The CKM unitarity triangle.

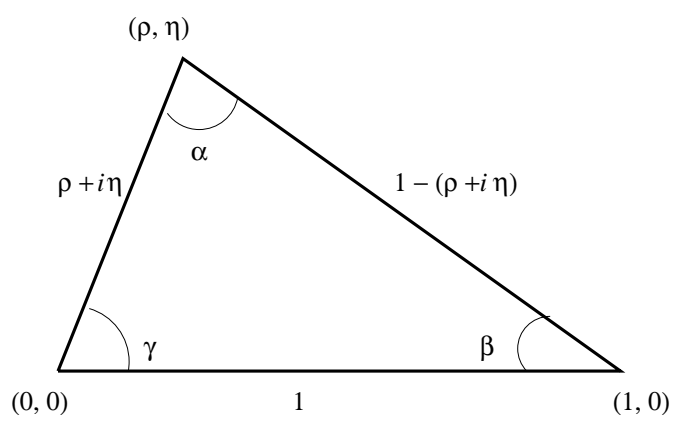

operation can define a symmetry between interactions that behave the same with and without the application of charge conjugation. Another such discrete symmetry operation is the inversion of spatial coordinates knows as parity $(P)$. The weak interaction is observed to violate $P$. This can be understood most easily by the presence of only left-handed neutrinos. Under parity the handedness of space is reversed. If $P$ were a good symmetry of the weak interaction, right-handed neutrinos would exist and have the same interactions as the left-handed versions that we do observe in nature. Similarly for $C$, all anti-neutrinos are right-handed and there are no known left-handed anti-neutrinos in nature.

The combined operation of $C$ and $P$ together, however, is very nearly a good symmetry of the weak interaction (and so far is thought to be an exact symmetry of the strong and electromagnetic interactions). Hence a left-handed neutrino can be turned into a right-handed anti-neutrino with the application of $C$ and $P$ and both particles are observed to exist. In 1964 the first violation of the symmetry of the combined operation $C P$ was discovered in the neutral kaon system [8]. It has since been discovered in neutral $B$ meson interactions at $B$ factory experiments such as BABAR [9].

Mathematically, the $C P$ operation transforms the particle fields and operators into their hermitian conjugates. This gives the desired properties of the discrete symmetry operations and preserves the overall hermiticity of the theory. One effect of this 
transformation is that any non-trivial phase in the theory will change sign and such a phase is exactly what we found to be present in the three quark mixing model described by the CKM matrix. This gives the SM a mechanism to allow for non-zero $C P$ violation.

\section{$1.4 \quad C P$ Violation in $B^{0}$ Mesons}

Quark flavor is conserved in electromagnetic and strong interactions and quarks created in weak interactions are produced in weak eigenstates. $B^{0}$ mesons contain a $\bar{b}$ quark and another lighter quark ( $\mathrm{d}$ or s). This analysis is concerned with the lightest of the $B^{0}$ mesons, those with a $\bar{b}$ quark paired with a d quark. Such decays are described by the Unitarity Triangle. The $B_{s}^{0}$ meson is also of interest and can be related to another of the unitarity relationships from the CKM matrix, though it is not discussed here.

The $B^{0}$ meson with definite quark content $(\bar{b} d)$ and its anti-particle, $\bar{B}^{0}(b \bar{d})$ can also be described in the mass eigenstate basis, or as is more commonly written,

$$
\begin{aligned}
\left|B_{L}\right\rangle & =p\left|B^{0}\right\rangle+q\left|\bar{B}^{0}\right\rangle \\
\left|B_{H}\right\rangle & =p\left|B^{0}\right\rangle-q\left|\overline{B^{0}}\right\rangle
\end{aligned}
$$

where $\left|B_{L}\right\rangle$ and $\left|B_{H}\right\rangle$ are the mass eigenstates denoting the "light" and "heavy" particles. Since $C P$ need not be conserved in weak interactions these two bases are not necessarily the same. The coefficients p and q are complex and obey the normalization,

$$
|q|^{2}+|p|^{2}=1
$$

These states evolve according to the time-dependent Schrodinger equation,

$$
i \frac{d}{d t}\left(\begin{array}{c}
p \\
q
\end{array}\right)=\mathbf{H}\left(\begin{array}{c}
p \\
q
\end{array}\right) \equiv\left(\mathbf{M}-\frac{i}{2} \boldsymbol{\Gamma}\right)\left(\begin{array}{c}
p \\
q
\end{array}\right) .
$$

This parameterization is useful because, while $\mathbf{H}$ cannot be Hermitian because the $B^{0} / \bar{B}^{0}$ will eventually decay, $\mathbf{M}$ and $\boldsymbol{\Gamma}$ are Hermitian $2 \times 2$ complex matrices. 
The two physical states, $\left|B_{L}\right\rangle$ and $\left|B_{H}\right\rangle$, have different lifetimes as well as masses, defined as

$$
\begin{aligned}
\Delta m_{B} & \equiv M_{H}-M_{L}, \\
\Delta \Gamma_{B} & \equiv \Gamma_{H}-\Gamma_{L} .
\end{aligned}
$$

Solving for the eigenvalues of Eq. 1.8 we obtain [6]

$$
\mu^{ \pm}=M_{11}-\frac{i}{2} \Gamma_{11} \pm \frac{q}{p}\left(M_{12}-\frac{i}{2} \Gamma_{12}\right),
$$

with the ratio $\left(\frac{q}{p}\right)^{2}$ given by,

$$
\left(\frac{q}{p}\right)^{2}=\frac{M_{12}^{*}-\frac{i}{2} \Gamma_{12}^{*}}{M_{12}-\frac{i}{2} \Gamma_{12}}
$$

Using the form of $\left|B_{L}\right\rangle$ from Eq. 1.5 and the eigenvalues in Eq. 1.11, the Schrodinger equation gives,

$$
\left(\mathbf{M}-\frac{i}{2} \boldsymbol{\Gamma}\right)\left(\begin{array}{c}
p \\
q
\end{array}\right)=\left[M_{11}-\frac{i}{2} \Gamma_{11}-\frac{q}{p}\left(M_{12}-\frac{i}{2} \Gamma_{12}\right) \mid\right]\left(\begin{array}{c}
p \\
q
\end{array}\right) .
$$

Solving, we find,

$$
\begin{aligned}
M_{H}-\frac{i}{2} \Gamma_{H} & =\mu^{+}, \\
M_{L}-\frac{i}{2} \Gamma_{L} & =\mu^{-},
\end{aligned}
$$

or

$$
\begin{aligned}
\Delta m_{B} & =2 \operatorname{Re}\left(\frac{q}{p}\left(M_{12}-\frac{i}{2} \Gamma_{12}\right)\right) \\
\Delta \Gamma_{B} & =-4 \operatorname{Im}\left(\frac{q}{p}\left(M_{12}-\frac{i}{2} \Gamma_{12}\right)\right) .
\end{aligned}
$$

The time evolution of the mass eigenstates can be written

$$
\begin{aligned}
\left|B_{H}(t)\right\rangle & =e^{-i\left(M_{H}-\frac{i}{2} \Gamma_{H}\right) t}\left|B_{H}(0)\right\rangle, \\
\left|B_{L}(t)\right\rangle & =e^{-i\left(M_{L}-\frac{i}{2} \Gamma_{L}\right) t}\left|B_{L}(0)\right\rangle .
\end{aligned}
$$


The time evolution of the flavor eigenstates can then be constructed using Eqs. 1.5, 1.14 , and 1.18 , as

$$
\left|B_{\text {phys }}^{0}(t)\right\rangle=\frac{1}{2 p}\left[e^{-i \mu_{-} t}\left(p\left|B^{0}\right\rangle+q\left|\bar{B}^{0}\right\rangle\right)+e^{-i \mu_{+} t}\left(p\left|B^{0}\right\rangle-q\left|\bar{B}^{0}\right\rangle\right)\right]
$$

where $\left|B_{\text {phys }}^{0}(t)\right\rangle$ represents the time evolution of a neutral $B$ meson that is in a pure $B^{0}$ flavor state at time $\mathrm{t}=0$.

It is useful to simplify this expression by noting that the difference in widths, $\Delta \Gamma_{B}$ is expected to be negligibly small with,

$$
\Delta \Gamma_{B} / \Gamma_{B}=O\left(10^{-2}\right)[10]
$$

Only limits have been placed experimentally $\left(\left|\Delta \Gamma_{B} / \Gamma_{B}\right|<0.084[11]\right)$, but the width difference arises from decay channels common to $B^{0}$ and $\overline{B^{0}}$, which are found at or below the level of $10^{-3} . \Delta m_{B}$ is well measured and found to be $\frac{3}{4} \Gamma_{B}[2]$, which implies,

$$
\Delta \Gamma_{B} \ll \Delta m_{B}
$$

Explicitly replacing the eigenvalues from Eq. 1.20 we can write this oscillating time dependent state in terms of masses and lifetimes,

$$
\left|B_{\text {phys }}^{0}(t)\right\rangle=e^{-i(M-i \Gamma / 2) t}\left[\cos \left(\frac{\Delta m_{B} t}{2}\right)\left|B^{0}\right\rangle+i\left(\frac{q}{p}\right) \sin \left(\frac{\Delta m_{B} t}{2}\right)\left|\bar{B}^{0}\right\rangle\right]
$$

where $\Gamma=\Gamma_{L}=\Gamma_{H}$ and we have neglected the difference in width, and $M=\frac{1}{2}\left(M_{H}+\right.$ $\left.M_{L}\right)$. A similar expression can be written for $\left|\bar{B}_{\text {phys }}^{0}(t)\right\rangle$,

$$
\left|\bar{B}_{\text {phys }}^{0}(t)\right\rangle=e^{-i(M-i \Gamma / 2) t}\left[i\left(\frac{q}{p}\right) \sin \left(\frac{\Delta m_{B} t}{2}\right)\left|B^{0}\right\rangle+\cos \left(\frac{\Delta m_{B} t}{2}\right)\left|\bar{B}^{0}\right\rangle\right] .
$$

\subsection{Time Evolution of $B \bar{B}$ Pairs from $\Upsilon(4 S)$}

The time evolution of a single $B^{0}$ is as described above. As will be described in Chapter 2, a beneficial configuration for an accelerator experiment is to operate at the $\Upsilon(4 S)$ resonance, which decays to $B \bar{B}$ pairs in a coherent $\mathrm{L}=1$ state. The $B$ mesons 
evolve in phase, which means that at any point before one of the particles decays, there is always exactly one $B^{0}$ and one $\overline{B^{0}}$ meson. When one of the $B$ mesons decays, the other can be known to be in the conjugate state at that time. The longer-lived $B$ meson will continue to oscillate as described in Eq. 1.23, with opposite flavor at the time of the decay of the shorter-lived $B$ meson.

This coherence can be exploited to extract information from a time-dependent fit in a $B^{0}$ decay if the flavor of the other $B$ can be determined. This is referred to as "flavor tagging" or simply "tagging" and the "other $B$ " is referred to as $B_{\text {tag. The }}$ tagging is accomplished through a number of channels with the most reliable method coming from a semi-leptonic $B^{0}$ decay where the sign of the charged lepton indicates if it came from a $b$ or $\bar{b}$ quark. Similar tagging analysis can be accomplished with decays to $s$ quarks as well. A discussion of the effects of imperfect tagging is given in Sec. 3.5.4.

\subsection{Types of $C P$ Violation}

$C P$ violation can appear in the $B$ system through three mechanisms: in the decay of the $B$ meson; in the mixing of $B^{0}$ and $\bar{B}^{0}$ mesons; and in the interference between mixing and decay. Each type is considered here.

\subsection{1 $C P$ Violation in Decay}

$C P$ Violation in Decay, or Direct $C P$ Violation, occurs when the rates of $C P$ conjugate processes differ. This is possible when the phases from multiple decay paths interfere with each other. Two types of phases are relevant. The first type of phase arises from the presence of complex contributions in the decay amplitude. In the SM this can only occur in the weak interactions though quark mixing in the CKM matrix, so this phase is known as the "weak phase." As noted in Sec. 1.3 this phase has the opposite sign in the conjugate process. The second possible phase can arise from intermediate steps in the decay. These phases, which remain the same for conjugate processes, are known 
as the "strong phase" because of the strong processes which dominate the rescattering effects. For both types of phases, it is only a phase difference that can have physical significance.

We can then write the amplitude for each possible decay to final state $f$ as,

$$
\left.A_{f}=A e^{i(\delta+\phi}\right)
$$

where $\delta$ and $\phi$ are the strong and weak phases respectively. Since the sign of the weak phase changes for the conjugate process each conjugate term is of the form

$$
\left.\bar{A}_{\bar{f}}=e^{i \theta} A e^{i(\delta-\phi}\right),
$$

where the $e^{i \theta}$ is an overall phase arising from the fact that the mass eigenstates are not necessarily $C P$ eigenstates. This overall phase has no physical importance. When only a single amplitude for a given decay and its charge conjugate is present taking the difference of the magnitudes squared gives

$$
\left|A_{f}\right|^{2}-\left|\bar{A}_{\bar{f}}\right|^{2}=\left|A e^{i(\delta+\phi)}\right|^{2}-\left|e^{i \theta} A e^{i(\delta-\phi)}\right|^{2}=A^{2}-A^{2}=0,
$$

a result without $C P$ violation.

However, in the case of a decay with multiple amplitudes, interference is possible and can lead to $C P$ violation. The case with two amplitudes is given by [12],

$$
\begin{aligned}
\left|A_{f}\right|^{2}-\left|\bar{A}_{\bar{f}}\right|^{2} & =\left|A_{1} e^{i\left(\delta_{1}+\phi_{1}\right)}+A_{2} e^{i\left(\delta_{2}+\phi_{2}\right)}\right|^{2}-\left|e^{i \theta}\left(A_{1} e^{i\left(\delta_{1}-\phi_{1}\right)}+A_{2} e^{i\left(\delta_{2}-\phi_{2}\right)}\right)\right|^{2} \\
& =-4 A_{1} A_{2} \sin \left(\delta_{1}-\delta_{2}\right) \sin \left(\phi_{1}-\phi_{2}\right) .
\end{aligned}
$$

If either the strong or weak phase from the two processes is the same no $\operatorname{direct} C P$ violation will be present. A generalization to more than two amplitudes is possible as shown in [10].

The measurable quantity, independent of any phase convention, is $\left|\frac{\bar{A}_{\bar{f}}}{A_{f}}\right|$. The condition for $C P$ violation in decay can be written as,

$$
\left|\frac{\bar{A}_{\bar{f}}}{A_{f}}\right|=\left|\frac{\sum_{i} A_{i} e^{i\left(\delta_{i}-\phi_{i}\right)}}{\sum_{i} A_{i} e^{i\left(\delta_{i}+\phi_{i}\right)}}\right| \neq 1 .
$$


This is the only type of $C P$ violation possible for charged $B$ mesons. The results from measurements of this type usually are quoted as the charge asymmetry,

$$
A_{c h}=\frac{\Gamma\left(B^{-} \rightarrow f\right)-\Gamma\left(B^{+} \rightarrow \bar{f}\right)}{\Gamma\left(B^{+} \rightarrow f\right)+\Gamma\left(B^{-} \rightarrow \bar{f}\right)}=\frac{1-|\bar{A} / A|^{2}}{1+|\bar{A} / A|^{2}} .
$$

\subsection{2 $C P$ Violation in Mixing}

$C P$ violation in mixing, also referred to as indirect $C P$ violation, occurs when the mass eigenstates for a particle differ from the $C P$ eigenstates. From Eq. 1.12, the relevant measurable quantity independent of phase convention is the ratio,

$$
\left|\frac{q}{p}\right|^{2}=\left|\frac{M_{12}^{*}-\frac{i}{2} \Gamma_{12}^{*}}{M_{12}-\frac{i}{2} \Gamma_{12}}\right| .
$$

For $\left|\frac{q}{p}\right|=1$ the mass eigenstates are the same as the $C P$ eigenstates and no indirect $C P$ violation is present. Therefore, we can write the condition for indirect $C P$ violation as,

$$
\left|\frac{q}{p}\right| \neq 1
$$

\subsection{3 $C P$ Violation in the Interference Between Mixing and Decay}

This third type of $C P$ violation can only occur in decays to $C P$ eigenstates. If a given $C P$ eigenstate, $f_{C P}$, is accessible from both $B^{0}$ and $\bar{B}^{0}, C P$ violation is possible when the decay $B^{0} \rightarrow f_{C P}$ interferes with $B^{0} \rightarrow \bar{B}^{0} \rightarrow f_{C P}$.

As in the case of $C P$ violation in decay we can define a rate asymmetry like Equation 1.30. However here the $C P$ violation is manifestly a time dependent effect due to the oscillatory nature of the neutral meson system. We define a time-dependent asymmetry,

$$
A_{f_{C P}}=\frac{\Gamma\left(B_{\text {phys }}^{0}(t) \rightarrow f_{C P}\right)-\Gamma\left(\bar{B}_{\text {phys }}^{0}(t) \rightarrow f_{C P}\right)}{\Gamma\left(B_{\text {phys }}^{0}(t) \rightarrow f_{C P}\right)+\Gamma\left(\bar{B}_{\text {phys }}^{0}(t) \rightarrow f_{C P}\right)} .
$$

From the decay rates in Eq. 1.23, the decay rate distribution $f_{+}\left(f_{-}\right)$for $B_{C P} \rightarrow f$ 
when $B_{\text {tag }}$ is a $B^{0}\left(\bar{B}^{0}\right)$ is given by [10]

$$
f_{ \pm}(\Delta t)=\frac{e^{-|\Delta t| / \tau}}{4 \tau}\left[1 \pm S_{f_{C P}} \sin \left(\Delta m_{B} \Delta t\right) \mp C_{f_{C P}} \cos \left(\Delta m_{B} \Delta t\right)\right]
$$

where $\tau$ is the mean lifetime of the $B^{0}\left(1 / \Gamma_{B}\right), \Delta t \equiv t_{C P}-t_{\mathrm{tag}}$, and $S_{f_{C P}}$ and $C_{f_{C P}}$ are defined as

$$
S_{f_{C P}} \equiv \frac{2 \operatorname{Im}\left\{\lambda_{f_{C P}}\right\}}{1+\left|\lambda_{f_{C P}}\right|^{2}}, \quad C_{f_{C P}} \equiv \frac{1-\left|\lambda_{f_{C P}}\right|^{2}}{1+\left|\lambda_{f_{C P}}\right|^{2}}
$$

The parameter $\lambda_{f_{C P}}$ is defined by

$$
\lambda_{f_{C P}}=\frac{q \bar{A}_{f_{C P}}}{p A_{f_{C P}}}=\eta_{f_{C P}} \frac{q \bar{A}_{\bar{f}_{C P}}}{p A_{f_{C P}}},
$$

where $\eta_{f_{C P}}$ is the $C P$ eigenvalue of the state $f_{C P}$ and

$$
\bar{A}_{f_{C P}}=\eta_{f_{C P}} \bar{A}_{\bar{f}_{C P}}
$$

Making use of these definitions, the time-dependent $C P$ asymmetry is

$$
A_{f_{C P}}=\frac{f_{+}(\Delta t)-f_{-}(\Delta t)}{f_{+}(\Delta t)+f_{-}(\Delta t)}=S_{f_{C P}} \sin \left(\Delta m_{B} \Delta t\right)-C_{f_{C P}} \cos \left(\Delta m_{B} \Delta t\right) .
$$

From Section 1.6.1 if we assume no $C P$ violation in decay, then $\left|\frac{\bar{A}_{\bar{f}_{C P}}}{A_{f_{C P}}}\right|=1$; from Section 1.6.2 if we assume no $C P$ violation in mixing, then $\left|\frac{q}{p}\right|=1$. If either $C P$ violation in decay or mixing were present, $A_{f_{C P}} \neq 0$. However, in the case with no $C P$ violation in mixing or decay $\left(\left|\lambda_{f_{C P}}\right|=1\right)$, it is still possible to have $C P$ violation if

$$
\operatorname{Im}\left\{\lambda_{f_{C P}}\right\} \neq 0 \text {. }
$$

This is the condition for $C P$ violation in the interference between mixing and decay. Stated differently, $C P$ violation in the interference between mixing and decay is present if $S_{f_{C P}} \neq 0$.

\subsection{CKM Contribution from $B \bar{B}$ Mixing}

In the Standard Model $B^{0} / \bar{B}^{0}$ mixing occurs through box diagrams such as the one shown in Fig. 1.2. While all up-type quarks technically may participate in the loop, 


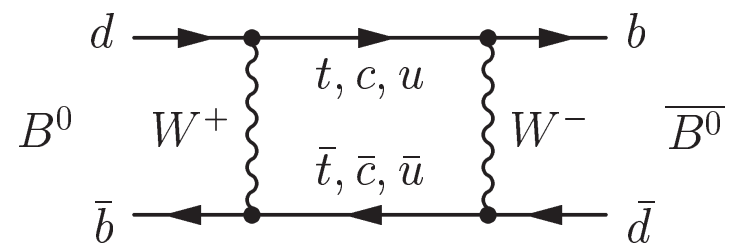

Figure 1.2: Box diagram describing $B^{0}-\bar{B}^{0}$ mixing in the SM.

the amplitude is proportional to $m_{q}^{2}$ and the top quark term dominates. The relevant quark transitions are $d \rightarrow t \rightarrow b$ and $\bar{b} \rightarrow \bar{t} \rightarrow \bar{d}$, giving the CKM matrix element factors $\frac{V_{t b}^{*} V_{t d}}{V_{t b} V_{t d}^{*}}$. With the assumption, $\Delta \Gamma_{B} \ll \Delta m_{B}$ (Eq. 1.22), this can be written,

$$
\frac{q}{p}=\frac{V_{t b}^{*} V_{t d}}{V_{t b} V_{t d}^{*}}
$$

where we have omitted an arbitrary phase factor.

The cleanest extraction of theoretical parameters describing $C P$ violation from the CKM matrix in the $\mathrm{SM}$ is from $C P$ violation in the interference between mixing and decay, where one decay path proceeds through $B$ mixing and another does not. The "golden" example of such a decay channel is $B^{0} \rightarrow J / \psi K_{S}^{0}$ which proceeds predominately via the CKM-favored (though color-suppressed) $b \rightarrow c$ tree amplitude shown in Fig. 1.3.

If we assume no $C P$ violation in mixing or decay and require $\left|\lambda_{f_{C P}}\right|=1$ then Eq. 1.38 reduces to

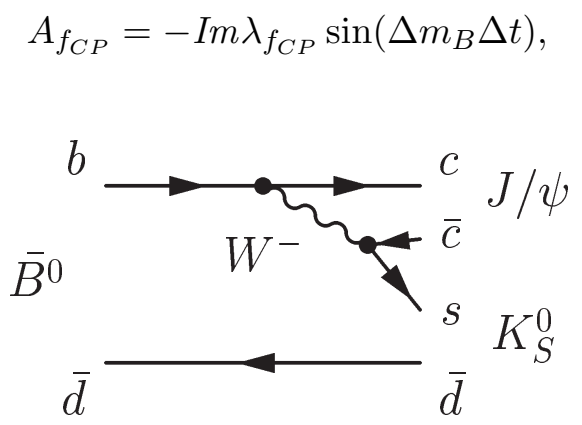

Figure 1.3: Tree diagram describing the decay $\bar{B}^{0} \rightarrow J / \psi K_{S}^{0}$. 
where we have shown $\lambda_{f_{C P}}=\eta_{f_{C P}} \frac{q}{p} \frac{\bar{A}_{\bar{f}_{C P}}}{A_{f_{C} P}}$ in Eq 1.36. $\frac{q}{p}$ is given in Eq. 1.40. $\frac{\bar{A}_{\bar{f}_{C P}}}{A_{f_{C} P}}$ has a factor of $\frac{V_{c b} V_{c s}^{*}}{V_{c b}^{*} V_{c s}}$ from the difference between the decay in Fig.1.3 and its conjugate process. Additionally, mixing between $K^{0}$ and $\overline{K^{0}}$ is required for the interference. A box diagram for mixing in the $K^{0}$ system similar to that of the $B^{0}$ gives a contribution of $\frac{V_{c s} V_{c d}^{*}}{V_{c s}^{*} V_{c d}}$.

Therefore, we have

$$
\frac{\bar{A}_{\bar{f}_{C P}}}{A_{f_{C} P}}=\left(\frac{V_{c b} V_{c s}^{*}}{V_{c b}^{*} V_{c s}}\right)\left(\frac{V_{c s} V_{c d}^{*}}{V_{c s}^{*} V_{c d}}\right),
$$

again omitting the arbitrary phases. This gives

$$
\lambda_{f_{C P}}\left(\psi K_{S}^{0}\right)=\eta_{\psi K_{S}^{0}}\left(\frac{V_{t b}^{*} V_{t d}}{V_{t b} V_{t d}^{*}}\right)\left(\frac{V_{c b} V_{c s}^{*}}{V_{c b}^{*} V_{c s}}\right)\left(\frac{V_{c s} V_{c d}^{*}}{V_{c s}^{*} V_{c d}}\right) .
$$

Canceling factors and inserting $\eta_{\psi K_{S}^{0}}=-1$, we find

$$
\lambda_{f_{C P}}\left(\psi K_{S}^{0}\right)=-\left(\frac{V_{t b}^{*} V_{t d}}{V_{t b} V_{t d}^{*}}\right)\left(\frac{V_{c b} V_{c d}^{*}}{V_{c b}^{*} V_{c d}}\right)=-\left(\frac{V_{c d}^{*} V_{c b}}{V_{t b} V_{t d}^{*}}\right)\left(\frac{V_{t d} V_{t b}^{*}}{V_{c b}^{*} V_{c d}}\right) .
$$

With $\beta=\arg \left[-\frac{V_{c d} V_{c b}^{*}}{V_{t d} V_{t b}^{*}}\right]$ from Eq.1.4, we can write $-\frac{V_{c d} V_{c b}^{*}}{V_{t d} V_{t b}^{*}}$ as $a e^{i \beta}$, with $a, \beta$ real and

$$
\lambda_{f_{C P}}\left(\psi K_{S}^{0}\right)=\frac{-a e^{-i \beta}}{a e^{i \beta}},
$$

which gives

$$
\operatorname{Im} \lambda_{f_{C P}}=\sin 2 \beta .
$$

Therefore, Eq. 1.41 gives

$$
A_{f_{C P}}(\Delta t)=\sin 2 \beta \sin \left(\Delta m_{B} \Delta t\right)
$$

Fitting for the amplitude of the sine component of the time-dependent $C P$ asymmetry allows for the extraction of $\sin 2 \beta$.

Diagrams with different weak phases for $B^{0} \rightarrow J / \psi K_{S}^{0}$ are suppressed by at least $O\left(\lambda^{2}\right)$ and are independent of any assumptions about factorization, color suppression or final state interactions. The theoretical uncertainty on the relationship of $S_{J / \psi K_{S}^{0}}$ to $\sin 2 \beta$ is found to be negligible [13]. 
The value of $\sin 2 \beta$ from $B^{0} \rightarrow J / \psi K_{S}^{0}$ and other $B^{0} \rightarrow c \bar{c} K^{0}$ final states has been measured by BABAR [14] and Belle [15] with a current world average of $\sin 2 \beta=$ $0.678 \pm 0.022 \pm 0.014[16]$, where the first uncertainty is statistical and the second is systematic.

\section{8 $C P$ Violation in $B^{0} \rightarrow \eta^{\prime} K_{S}^{0}$ and $\sin 2 \beta$}

The decay $B^{0} \rightarrow \eta^{\prime} K_{S}^{0}$, with $\eta_{\eta^{\prime} K_{S}^{0}}=-1$, can be analyzed in a similar fashion. However, the theoretical case is not as clear. Fig. 1.4 shows the Feynman diagrams for some possible decay amplitudes for $B^{0} \rightarrow \eta^{\prime} K^{0}$.

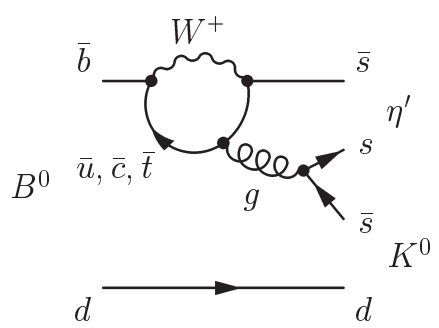

(a)

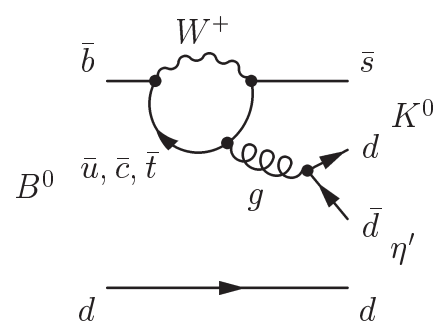

(b)

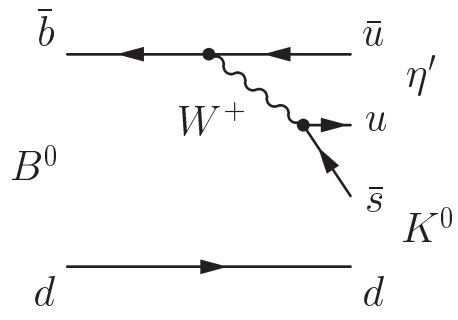

(c)

Figure 1.4: Feynman diagrams describing the decay $B^{0} \rightarrow \eta^{\prime} K^{0}$ via (a,b) internal gluonic penguin and (c) color-suppressed tree.

The presence of multiple diagrams complicates the extraction of $\sin 2 \beta$. In $B^{0} \rightarrow$ $\eta^{\prime} K_{S}^{0}$ the loop (penguin) diagrams are expected to dominate $[17,18]$. The penguin diagrams with t, c, and u quarks in the loop and the color and Cabibbo-suppressed tree diagram can be grouped using Unitarity relationships into a leading contribution of $O\left(\lambda^{2}\right)$ with the CKM factor $V_{c b} V_{c s}^{*}$ and secondary contribution of $O\left(\lambda^{4}\right)$ with the CKM factor $V_{u b} V_{u s}^{*}[10]$. Other diagrams, including electroweak penguins, can contribute in principle, but are expected to be even smaller. Ignoring the $O\left(\lambda^{4}\right)$ terms (the tree and u-loop penguin contributions), the interpretation of $\lambda_{f_{C P}}$ and $A_{f_{C P}}$ follows as in the $J / \psi K_{S}^{0}$ case to conclude

$$
S_{\eta^{\prime} K_{S}^{0}} \approx \sin 2 \beta
$$


Within the SM, predictions for the size of the contribution from non-leading-order diagrams and deviation of $\Delta S_{\eta^{\prime} K_{S}^{0}} \equiv S_{\eta^{\prime} K_{S}^{0}}-\sin 2 \beta$ from zero have been made using a variety of methods. Flavor SU(3) relationships among the branching fractions for decays of $B^{0}$ mesons to two-body final states containing $\eta, \eta^{\prime}$, and $\pi^{0}$ mesons have been used to evaluate the relative importance of different diagrams for $B^{0} \rightarrow \eta^{\prime} K_{S}^{0}[19,20]$. The relationships are used to place limits on $\Delta S_{\eta^{\prime} K_{S}^{0}}$. An updated version of this calculation estimates upper bounds of $-0.05<\Delta S_{\eta^{\prime} K_{S}^{0}}<0.10$ with a best guess value $\sim+0.02$ [21].

A second fruitful approach for calculating $\Delta S_{\eta^{\prime} K_{S}^{0}}$ in the SM comes from using a QCD factorization framework. An update to this result calculated at next-to-leading order finds $\Delta S_{\eta^{\prime} K_{S}^{0}}=+0.01 \pm 0.01[22]$, in nice agreement with the result from flavor $\mathrm{SU}(3)$. A recent calculation using soft-collinear effective theory [23] calculates $\Delta S_{\eta^{\prime} K_{S}^{0}}=$ $-0.02 \pm 0.01$, also in good agreement with the other theoretical results.

These calculations seek to establish the value of $\Delta S_{\eta^{\prime} K_{S}^{0}}$ in the SM. Any further deviation from $\sin 2 \beta$ could be the result of non-SM physics. In particular, the loop in the dominant penguin diagrams leaves open the possibility to observe indirectly the effects from additional diagrams with heavy non-SM particles participating in the loop.

\subsection{Branching Fractions in $B \rightarrow \eta^{\prime} K$ Decays}

The branching fractions for $B \rightarrow \eta^{\prime} K$ decays have long been noticed to be much larger than naively predicted $[24,25]$. The CKM suppression of all charmless $B$ meson decays classifies them as "rare decays" with typical branching fractions $\sim(1-10) \times 10^{-6}$. Both the charged and neutral $B \rightarrow \eta^{\prime} K$ branching fractions were found to be about an order of magnitude larger. In particular, they are found to be about a factor of 6 larger than $B \rightarrow \pi^{0} K$, which naively would appear to have similar contributing diagrams [26]. The enhanced branching fraction now appears to be the result of constructive interference between the two leading-order penguin diagrams (Fig. 1.4 a,b) as suggested by [27] and shown quantitatively in a NLO QCD factorization calculation by [18]. These two 
penguin diagrams have the same strong and weak phases, and therefore the interference does not affect the $A_{f_{C P}}$ other than to enhance the size of signals possible with the large branching fraction.

The charged decay $B^{+} \rightarrow \eta^{\prime} K^{+}$[28] has a similar set of Feynman diagrams to the neutral decay shown in Fig. 1.4 with the additional possibility of an external (color allowed) tree. Since the tree diagrams do not contribute at leading order, the branching fractions for the charged and neutral modes are expected to be similar.

Different strong phases between the penguin and tree processes would allow direct $C P$ violation possible in the charged decays as measured by the time-integrated decay rate difference between the two charged conjugate states, $\mathcal{A}_{c h}$. Penguin dominance causes the expected interference to be small and as a result the expected $\mathcal{A}_{c h}$ is quite small $[18,29]$.

\subsection{0 $C P$ Violation in $B^{0} \rightarrow \omega K_{S}^{0}$ and $\sin 2 \beta$}

There exists a whole series of $B^{0}$ decays to charmless $C P$ eigenstates via dominant penguin $b \rightarrow s s \bar{s}$ or $b \rightarrow s d \bar{d}$ diagrams. Other examples include $B^{0} \rightarrow \phi K_{S}^{0}, \pi^{0} K_{S}^{0}, \rho^{0} K_{S}^{0}$ etc. Each approximately measures $\sin 2 \beta$ in the SM (though $\Delta S$ must be calculated for each channel individually) and has the potential to deviate from $\sin 2 \beta$ due to non-SM effects.

This thesis includes a measurement of $A_{f_{C P}}$ for the decay $B^{0} \rightarrow \omega K_{S}^{0}$, with $\eta_{\omega K_{S}^{0}}=-1$, for which some additional detail is presented here. The Feynman diagrams are similar to those of $\eta^{\prime} K_{S}^{0}$, though only one color suppressed penguin is possible for the $b \rightarrow s d \bar{d}$ transition. The dominant penguin and first-order tree diagrams are shown in Fig. 1.5.

To leading order in the SM, $S_{\omega K_{S}^{0}}=\sin 2 \beta$, but contributions from the tree and u-loop penguin diagrams lead to a non-zero $\Delta S_{\omega K_{S}^{0}}$. The contribution from the tree is expected to be non-negligible as in the $B^{0} \rightarrow \pi^{0} K_{S}^{0}$ case, with the same Feynman 


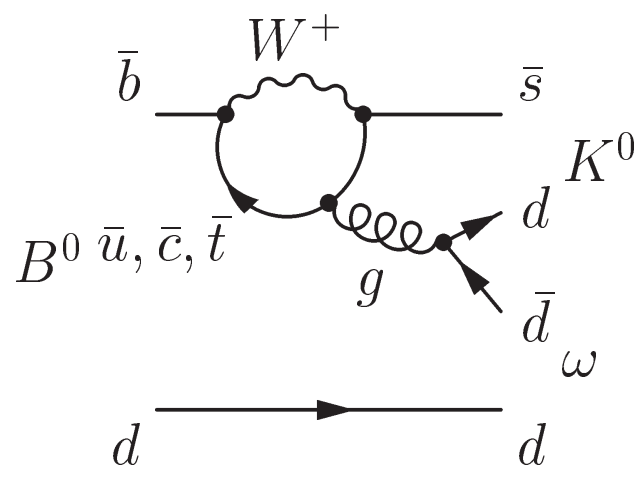

(a)

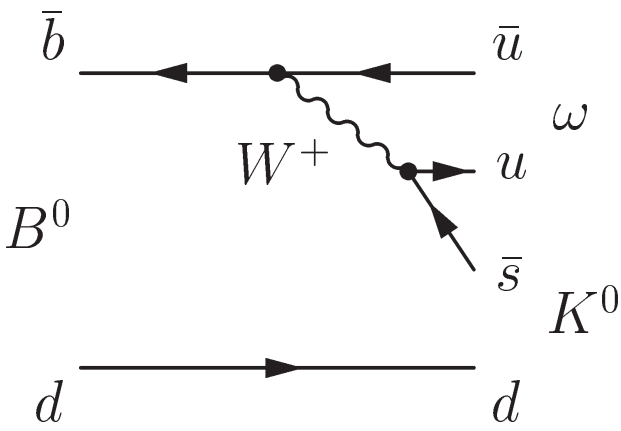

(b)

Figure 1.5: Feynman diagrams describing the decay $B^{0} \rightarrow \omega^{\prime} K^{0}$ via (a) internal gluonic penguin and (b) color-suppressed tree.

diagrams, where the flavor $\mathrm{SU}(3)$ approach predicts $\Delta S \lesssim+0.2$ [30]. The QCD factorization approach calculates a value of $\Delta S_{\omega K_{S}^{0}}=+0.13 \pm 0.08$ [22]. The QCD factorization approach in [22], however, fails to account for long-distance effects. A recent calculation [31] shows that the effects from final state interactions (FSI) are expected to be non-neglidable for $\omega K_{S}^{0}$ with the effect of lowering $\Delta S$. Accounting for both short distance effects (through QCD factorization) and FSI the authors calculate $\Delta S_{\omega K_{S}^{0}}=+0.01_{-0.04}^{+0.03}$

\subsection{Branching Fractions in $B \rightarrow \omega K$ and $B \rightarrow \omega \pi$ Decays}

The Feynman diagrams for the $\omega K / \pi$ system are the same as those for the $\pi^{0} K / \pi$ system. The leading contributions for the charged modes are shown in Fig. 1.6. The $B^{+} \rightarrow \omega \pi^{+}$decay is expected to be dominated by the tree diagram as shown in Fig. 1.6(a). The $B^{+} \rightarrow \omega K^{+}$decay is interesting since there are cancellations between dominant Wilson coefficients for the penguin (Fig. 1.6(c)) which would normally dominate the process [32]. The tree diagram as shown in Fig. 1.6(b) also contributes causing the potential for enhanced interference between the diagrams. The penguin diagram for the $B^{0} \rightarrow \omega K_{S}^{0}$ decay is shown in Fig. 1.5(a). The only tree diagram for this decay, shown 
in Fig. 1.5(b), is Cabibbo and color suppressed, though it is expected to contribute at some level as indicated by the $\Delta S_{\omega K_{S}^{0}} \neq 0$ prediction from short-distance effects. One recent calculation of pseudoscalar-vector modes with QCD factorization [32] predicts branching fractions of $4.9,5.9$ and $8.4 \times 10^{-6}$ for the $B^{0} \rightarrow \omega K_{S}^{0}, B^{+} \rightarrow \omega K^{+}$and $B^{+} \rightarrow \omega \pi^{+}$decays. The $\mathcal{A}_{c h}$ for the charged modes is expected to be small.

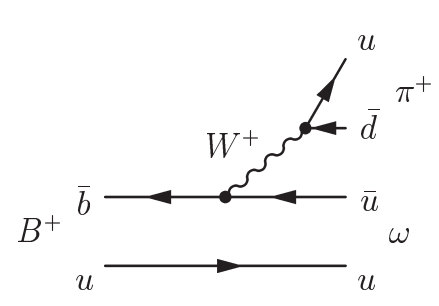

(a)

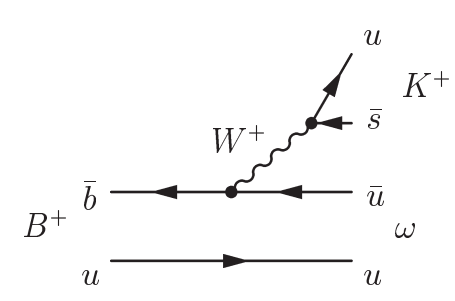

(b)

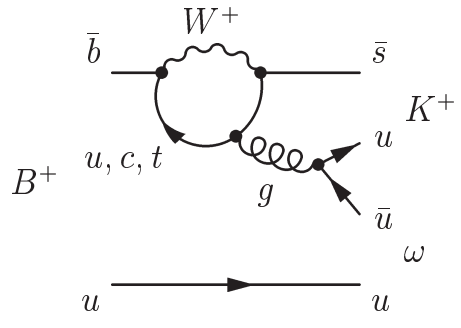

(c)

Figure 1.6: Feynman diagrams describing the decays $B^{+} \rightarrow \omega \pi^{+}$via (a) external tree and $B^{+} \rightarrow \omega K^{+}$via (b) external tree and (c) internal gluonic penguin.

\subsection{Previous Results}

This thesis presents updated results from measurements of $B$ decays to the final states $\eta^{\prime} K^{+}, \eta^{\prime} K^{0}, \omega \pi^{+}, \omega K^{+}$, and $\omega K^{0}$. Branching fractions are measured for all modes, charge asymmetries are measured for the charged modes and time-dependent $C P$-violating asymmetries are measured for the neutral modes. Table 1.2 presents the previous results for these measurements from CLEO [33][34][35], BABAR [36][37], and Belle [38][39][40]. 
Table 1.2: Summary of previous results for branching fraction in units of $10^{-6}$.

\begin{tabular}{cccc}
\hline \hline & CLEO [33][34][35] & BABAR [36][37] & Belle [38][39][40] \\
\hline $\mathcal{B}\left(\times 10^{-6}\right)$ & & & \\
$\eta^{\prime} K^{+}$ & $80_{-9}^{+10} \pm 7$ & $68.9 \pm 2.0 \pm 3.2$ & $69.2 \pm 2.2 \pm 3.7$ \\
$\eta^{\prime} K^{0}$ & $89_{-16}^{+18} \pm 9$ & $67.4 \pm 3.3 \pm 3.2$ & $58.9_{-3.5}^{+3.6} \pm 4.3$ \\
$\omega \pi^{+}$ & $11.3_{-2.9}^{+3.3} \pm 1.4$ & $6.1 \pm 0.7 \pm 0.4$ & $6.9 \pm 0.6 \pm 0.5$ \\
$\omega K^{+}$ & $3.2_{-1.9}^{+2.4} \pm 0.8$ & $6.1 \pm 0.6 \pm 0.4$ & $8.1 \pm 0.6 \pm 0.6$ \\
$\omega K^{0}$ & $10.0_{-4.2}^{+5.4} \pm 1.4$ & $6.2 \pm 1.0 \pm 0.4$ & $4.4_{-0.7}^{+0.8} \pm 0.4$ \\
\hline $\mathcal{A}_{c h}$ & & & \\
$\eta^{\prime} K^{+}$ & $0.03 \pm 0.12 \pm 0.02$ & $0.03 \pm 0.03 \pm 0.01$ & $0.03 \pm 0.03 \pm 0.02$ \\
$\omega \pi^{+}$ & $-0.34 \pm 0.25 \pm 0.02$ & $-0.01 \pm 0.10 \pm 0.01$ & $-0.02 \pm 0.09 \pm 0.01$ \\
$\omega K^{+}$ & - & $0.05 \pm 0.09 \pm 0.01$ & $0.05_{-0.07}^{+0.08} \pm 0.01$ \\
\hline$S_{f_{C P}}$ & - & $0.30 \pm 0.14 \pm 0.02$ & $0.65 \pm 0.18 \pm 0.04$ \\
$\eta^{\prime} K^{0}$ & - & $0.51_{-0.39}^{+0.35} \pm 0.02$ & $0.76 \pm 0.65_{-0.16}^{+0.13}$ \\
$\omega K_{S}^{0}$ & - & $-0.21 \pm 0.10 \pm 0.02$ & $-0.19 \pm 0.11 \pm 0.05$ \\
$C_{f_{C P}}$ & $-0.55_{-0.26}^{+0.28} \pm 0.03$ & $0.27 \pm 0.48 \pm 0.15$ \\
$\eta^{\prime} K^{0}$ & - & & \\
$\omega K_{S}^{0}$ & - &
\end{tabular}




\section{Chapter 2}

\section{The BABAR Experiment}

\subsection{PEP-II and the $B$ Factory}

The BABAR experiment [41] operates at the Positron-Electron Project II (PEPII) [42] storage ring at the Stanford Linear Accelerator Center (SLAC). The experiment has been designed to produce $B$ mesons in large quantities and is thus often referred to as a "B factory." PEP-II utilizes a beam of electrons accelerated to $9.0 \mathrm{GeV}$ and a beam of positrons accelerated to $3.1 \mathrm{GeV}$ to produce collisions with a center-of-mass (CM) energy of $10.58 \mathrm{GeV}$. This CM energy is chosen as the mass of the $\Upsilon(4 S)$ resonance, an excited bound state of bottom and anti-bottom quarks, which decays exclusively to pairs of $B$ mesons, either charged $\left(B^{+} B^{-}\right)$or neutral $\left(B^{0} \bar{B}^{0}\right)$.

The beams are accelerated to asymmetric energies to produce a moving CM with $\beta \gamma=.56$ in the laboratory frame. This allows the measurement of the separation in decay vertices of the $B$ pairs, which can then be translated into a time difference in the decays. Measurement of this decay time difference is of central importance to the time-dependent $C P$ asymmetry measurements described here.

PEP-II was designed to achieve a luminosity of $3 \times 10^{33} \mathrm{~cm}^{-2} \mathrm{~s}^{-1}$. Outstanding performance surpassing design and a number of successful upgrades have allowed for the achievement of a peak luminosity of greater than $1.2 \times 10^{34} \mathrm{~cm}^{-2} \mathrm{~s}^{-1}$. A comparison of the design and current typical running conditions for PEP-II is shown in Table 2.1. The increased luminosity relative to design comes mostly from increased currents in the 
beams. The integrated luminosity totals for the lifetime of the experiment are shown in Fig. 2.1. While most data is recorded at the $\Upsilon(4 S)$ resonance, $\approx 12 \%$ is recorded at a $\mathrm{CM}$ energy $40 \mathrm{MeV}$ below the $\Upsilon(4 S)$ resonance to allow for studies of non-resonant backgrounds.

Table 2.1: PEP-II beam parameters. Values are given both for the design and for typical colliding beam operation in 2006. HER and LER refer to the high energy $e^{-}$and low energy $e^{+}$ring, respectively. $\sigma_{L x}, \sigma_{L y}$, and $\sigma_{L z}$ refer to the horizontal, vertical, and longitudinal rms size of the luminous region.

\begin{tabular}{lcc}
\hline Parameters & Design & Typical \\
\hline Energy HER/LER $(\mathrm{GeV})$ & $9.0 / 3.1$ & $9.0 / 3.1$ \\
Current HER/LER $(\mathrm{A})$ & $0.75 / 2.15$ & $1.7 / 2.9$ \\
\# of bunches & 1658 & 1722 \\
Bunch spacing $(\mathrm{ns})$ & 4.2 & 4.1 \\
$\sigma_{L x}(\mu \mathrm{m})$ & 110 & 110 \\
$\sigma_{L y}(\mu \mathrm{m})$ & 3.3 & 2.9 \\
$\sigma_{L z}(\mathrm{~mm})$ & 9 & 10 \\
Luminosity $\left(10^{33} \mathrm{~cm}^{-2} \mathrm{~s}^{-1}\right)$ & 3 & 10 \\
Luminosity $\left(\mathrm{pb}^{-1} / \mathrm{d}\right)$ & 135 & 700 \\
\hline
\end{tabular}

An electron beam is produced by thermal emission from a filament and drawn toward a linear accelerator (linac) for acceleration by an applied electric field. A portion of the accelerated electrons is drawn off and collided with a tungsten target producing $e^{+} e^{-}$pairs. The positrons are collected and sent back to be accelerated in the 3 -km-long linac. After partial acceleration, both beams are passed through damping rings, where the combination of synchrotron radiation and applied electric and magnetic fields damp out transverse motion from the beams. The beams are then fed back into the linac and accelerated to their collision energies. Bunches are sent from the linac into the PEP-II storage rings. Fig. 2.2 shows a schematic of the linac and storage rings.

PEP-II makes use of a series of magnets to steer the beams through the 2.2km-diameter storage ring. The beams collide head-on at interaction region 2 (IR2), where the BABAR detector is located. A pair of dipole magnets (B1) on either side of 


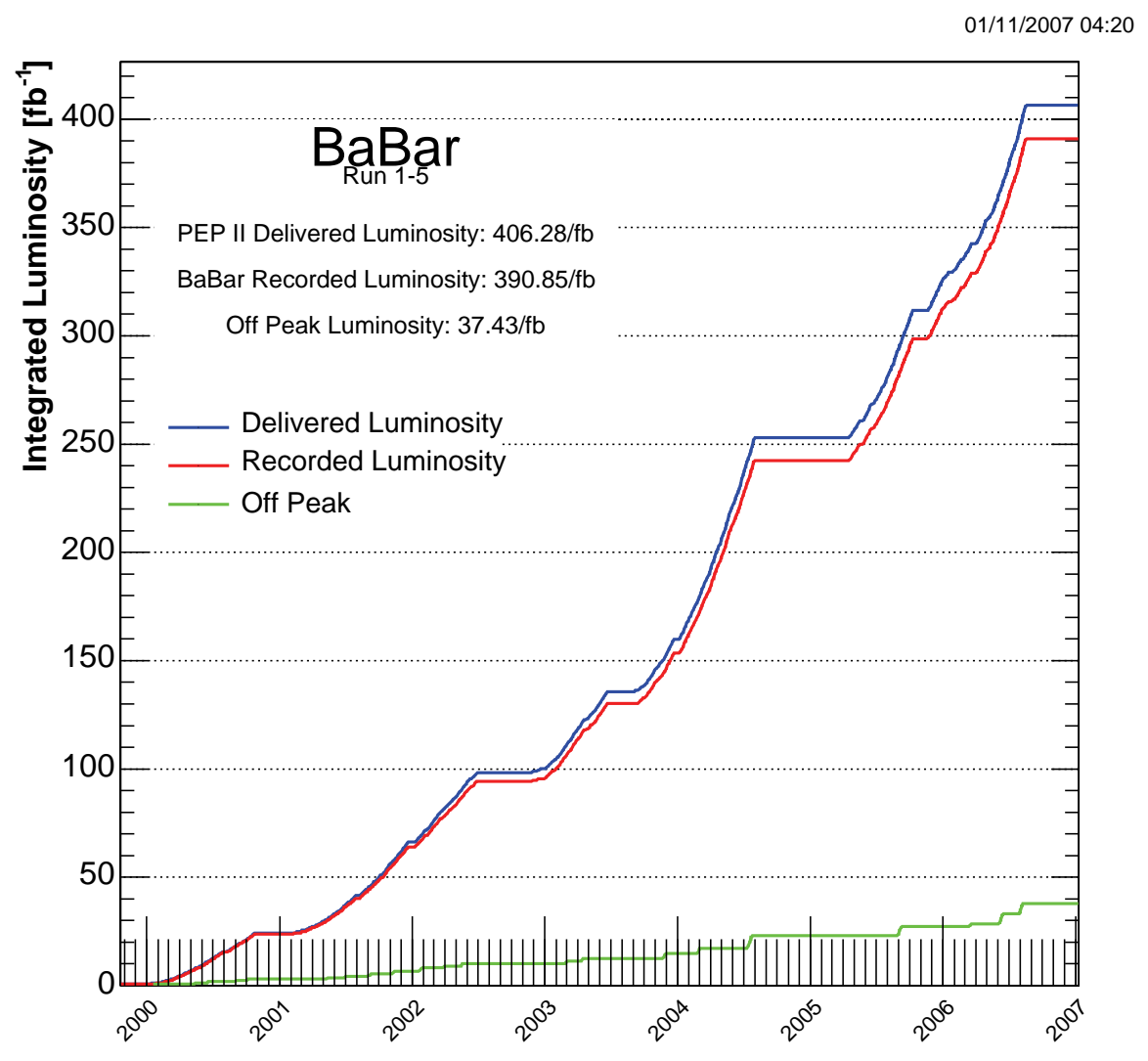

Figure 2.1: Total integrated luminosity delivered by PEP-II and recorded by BABAR over the lifetime of the experiment.

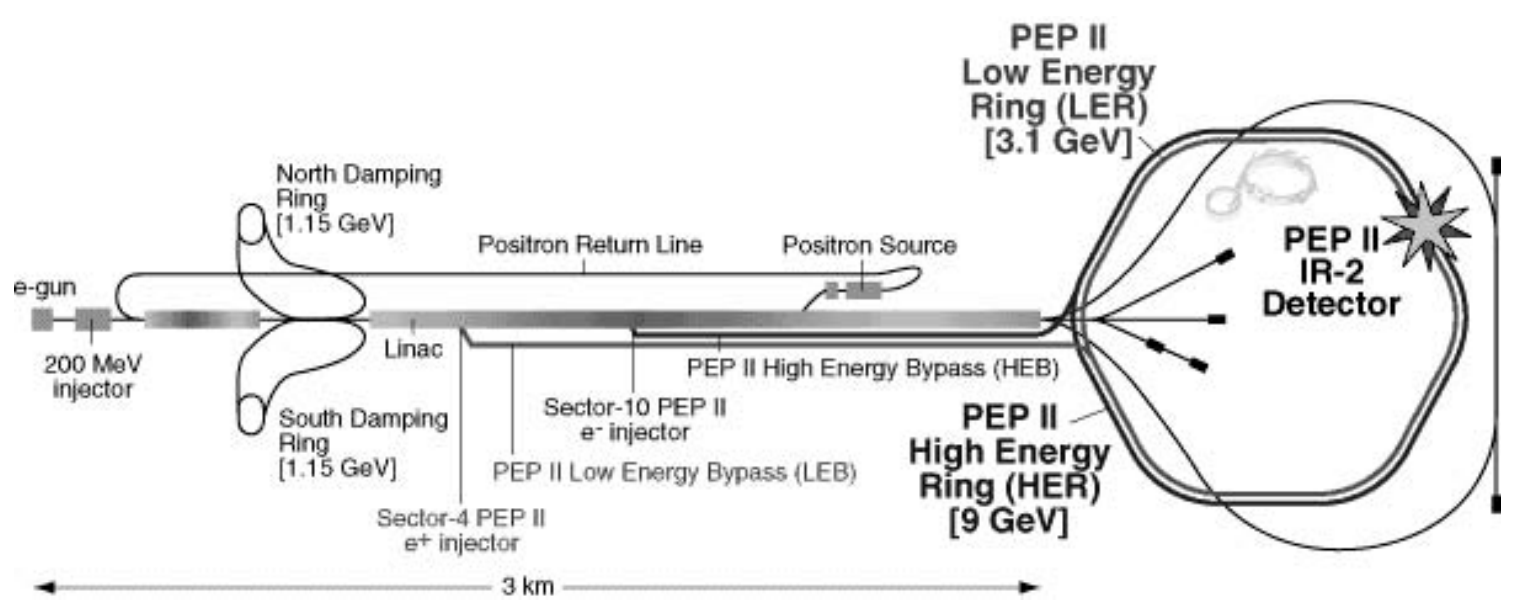

Figure 2.2: Schematic of the linac, PEP-II and IR-2. 
the $e^{+} e^{-}$interaction point (IP) is used to align the beams before collision and pairs of quadrapole magnets (Q1) provide final focusing in the vertical direction. After collision, the beams are separated magnetically in the horizontal plane by B1 followed by a series of quadrapole magnets beginning with the shared focusing Q1's (see Fig. 2.3) where they are returned to the storage rings to collide again.

The beam energies used in the collisions are calculated from the total magnetic field used to complete the PEP-II loop and the average deviation of the accelerating frequencies from their average values. The beam energies can be held stable to about 1 $\mathrm{MeV}$, though the uncertainty on the absolute measurements of the beam energy is 5-10 $\mathrm{MeV}$. A typical RMS energy spread for the low-energy beam (LER) is $2.3 \mathrm{MeV}$ and is 5.5 $\mathrm{MeV}$ for the high energy-beam (HER).

Variation of the $B \bar{B}$ pair production rate from the $\Upsilon(4 S)$ rate measured online provides an indication of drifting beam energies. Fully reconstructed $B$ meson decays provide the most accurate calibration of the absolute CM energy and can be used to recalibrate the beam energies.

The direction of the beams relative to the BABAR detector is measured with $e^{+} e^{-} \rightarrow \mu^{+} \mu^{-}$and $e^{+} e^{-} \rightarrow e^{+} e^{-}$events. Alignment uncertainties are the dominant uncertainty in the direction of the boost in the lab frame, with a typical uncertainty of less than one mrad.

The luminosity delivered is calculated based on the rate of $e^{+} e^{-} \rightarrow \mu^{+} \mu^{-}$and $e^{+} e^{-} \rightarrow e^{+} e^{-}$events as well as other QED processes, with an overall uncertainty of $1.1 \%$.

As shown in Table 2.1 the colliding beams are a factor of 30 smaller in the $y$ (vertical) dimension than the $x$ (horizontal) dimension and are much larger along the direction of motion. The horizontal size and position of the collision region can be measured by the distance of closest approach from two-track events. The vertical size is too small to measure directly, but can be inferred from the luminosity, beam currents 


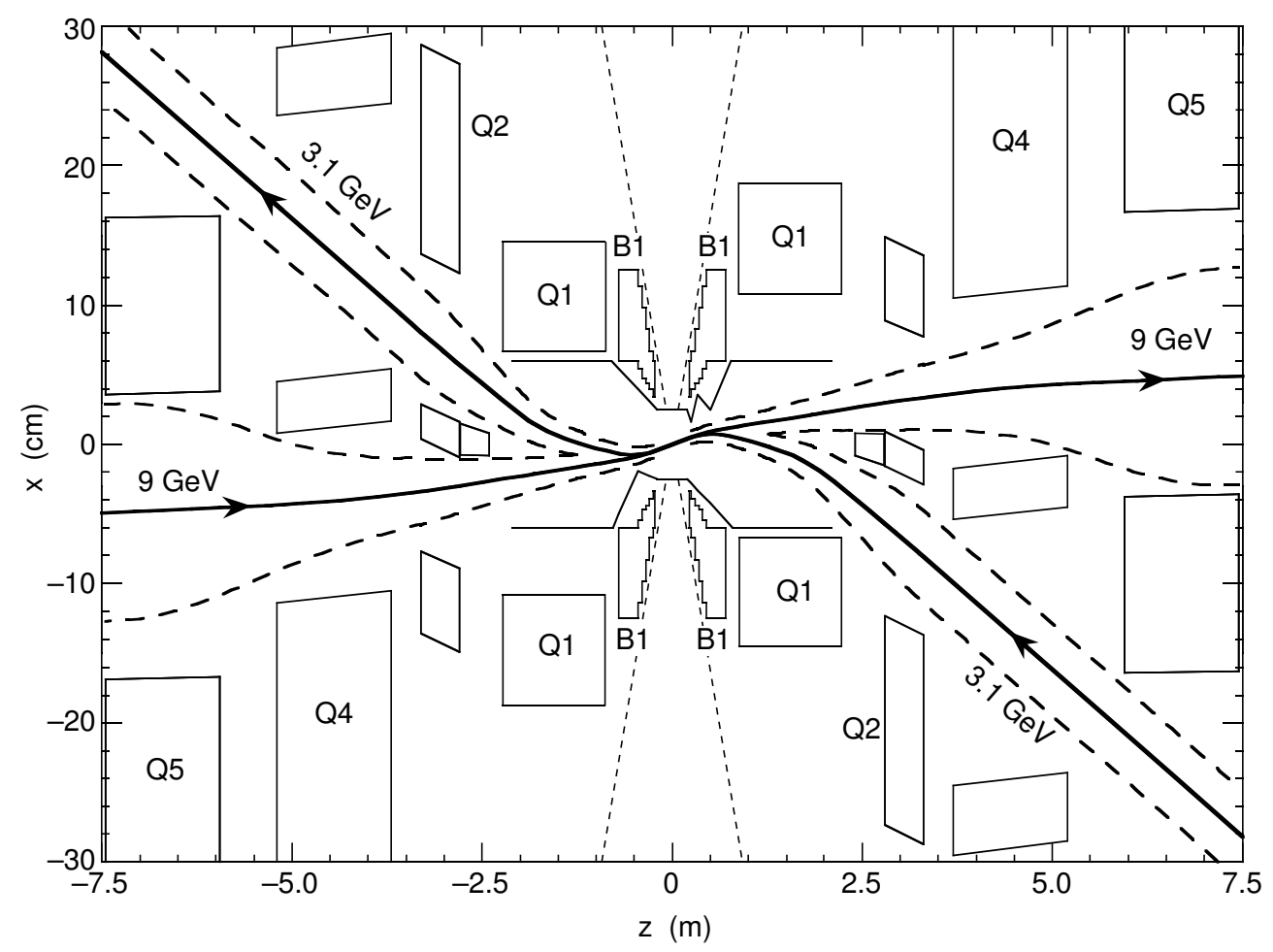

Figure 2.3: Close-up view of the beam crossing at the IP. The vertical scale is highly exaggerated to show the separation. The bend magnet (B1) separates the beams immediately after crossing and a series of quadrapole magnets (Q1-5) are used to stabilize and focus the beams as they are returned to the storage rings. 
and horizontal size.

\subsection{The BABAR Detector}

The BABAR detector is a multi-purpose detector consisting of a series of subsystems. Each subsystem allows for the detection and measurement of different particles required for BABAR analyses. The subsystems are arranged in layers enclosing the electron-positron interaction point. The design and performance of the BABAR detector components are discussed in this section.

A cross-sectional view of the BABAR detector with the layered subsystems labeled is shown in Fig. 2.4. A longitudinal view is shown in Fig. 2.5. A right-handed coordinate system is used with the principle axis of the main tracking system, a cylindrical drift chamber, defining the $z$-axis. This axis is very nearly the beam direction, with positive $z$ defined as the direction of the electron beam. The $y$-axis is defined as up, as shown in the figures. A $1.5 \mathrm{~T}$ magnetic field is supplied by a superconducting solenoid that surrounds the drift chamber. The whole detector is offset $0.37 \mathrm{~m}$ in the positive $z$ direction from the IP to maximize acceptance in the boosted CM.

\subsubsection{Silicon Vertex Tracker}

The inner-most detector subsystem is a silicon vertex tracker (SVT). It consists of a series of five double-sided layers of silicon strip detectors designed to measure the momentum and position of charged tracks. A multilayer design allows for accurate determination of the angle of the tracks passing through the magnetic field.

The three innermost layers are positioned as close to the beam pipe as possible at a radius of $3 \mathrm{~cm}$. A crucial design feature for the time-dependent asymmetry measurements is the ability to pinpoint the decay vertices of $B$ mesons in order to extract the time difference in their decays. The SVT has a resolution on the $z$-axis decay vertex for fully reconstructed $B$ mesons of $\sim 50 \mu \mathrm{m}$. 


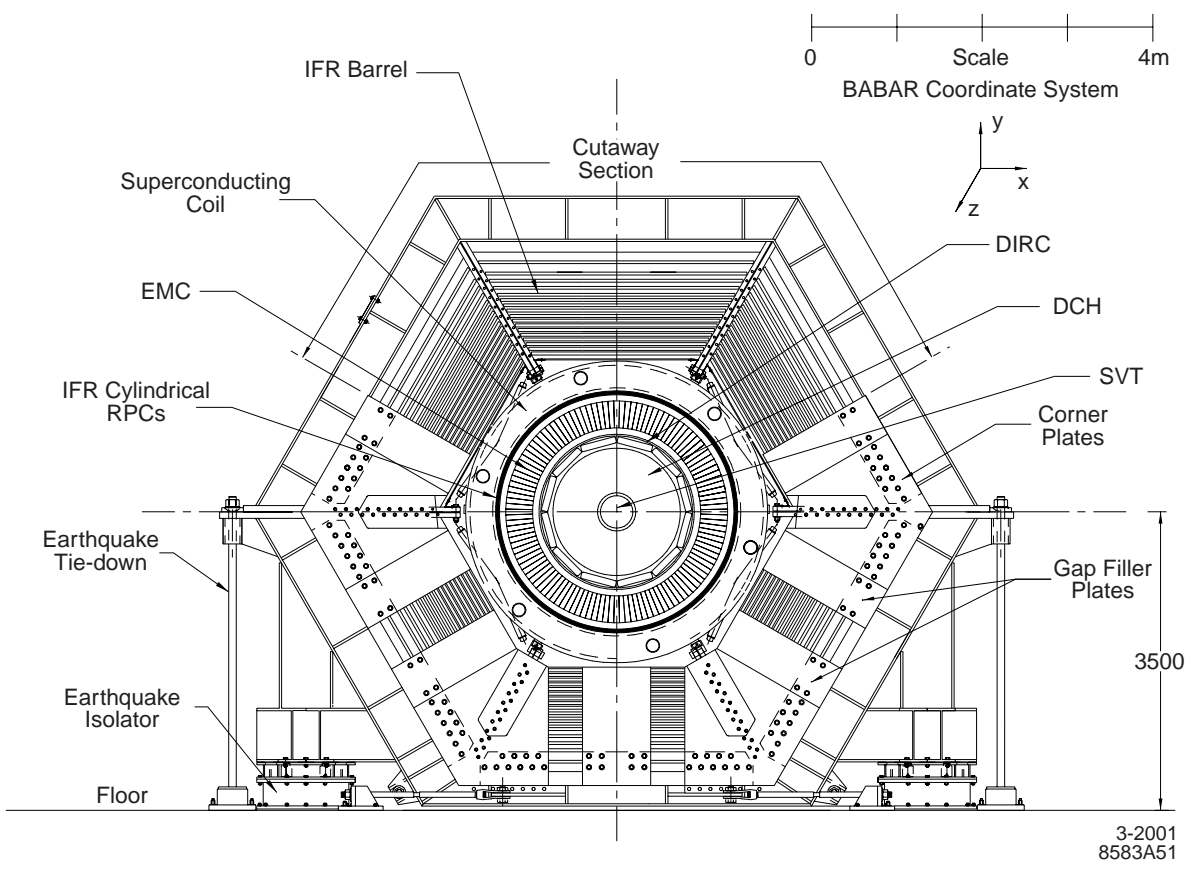

Figure 2.4: End view of the BABAR detector.

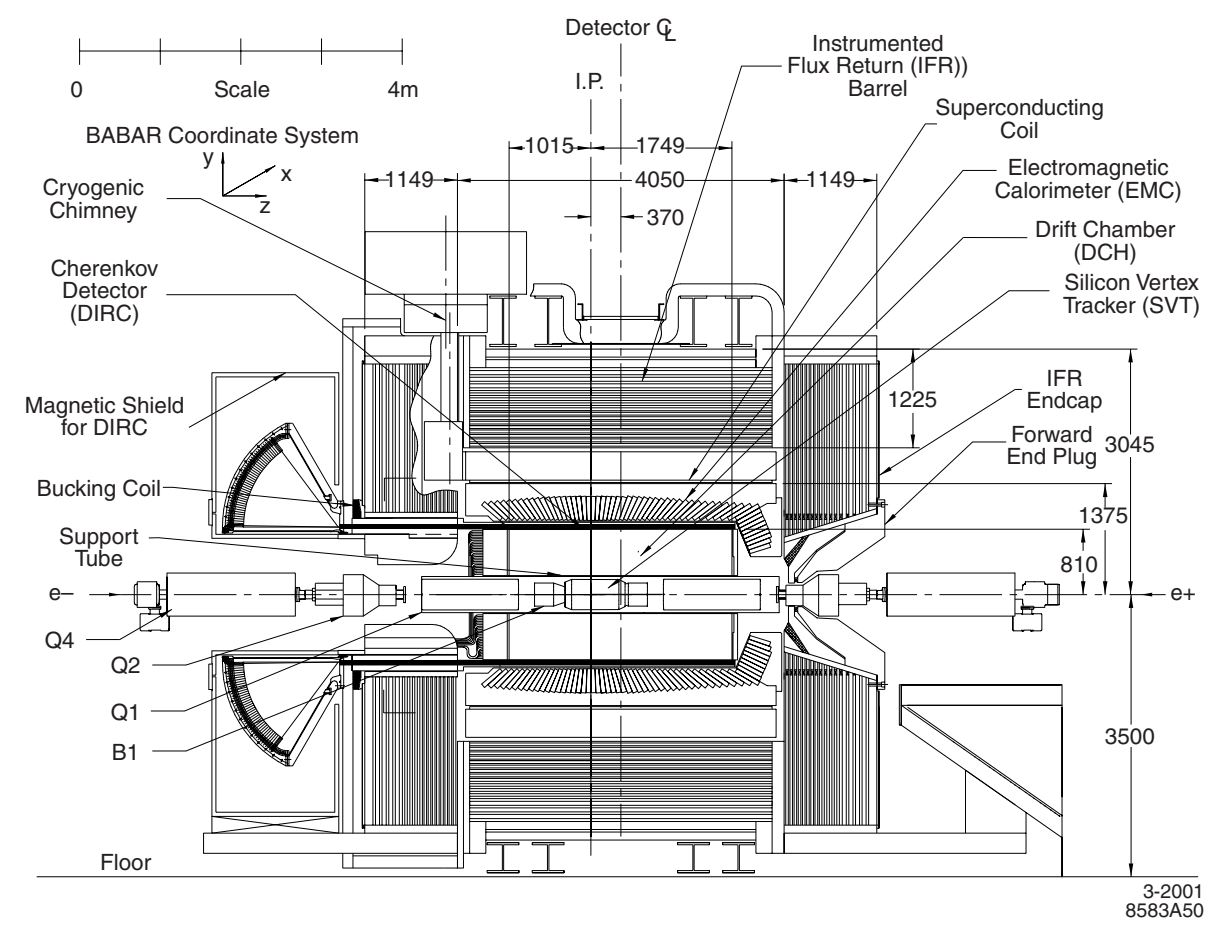

Figure 2.5: Longitudinal view of the BABAR detector. 


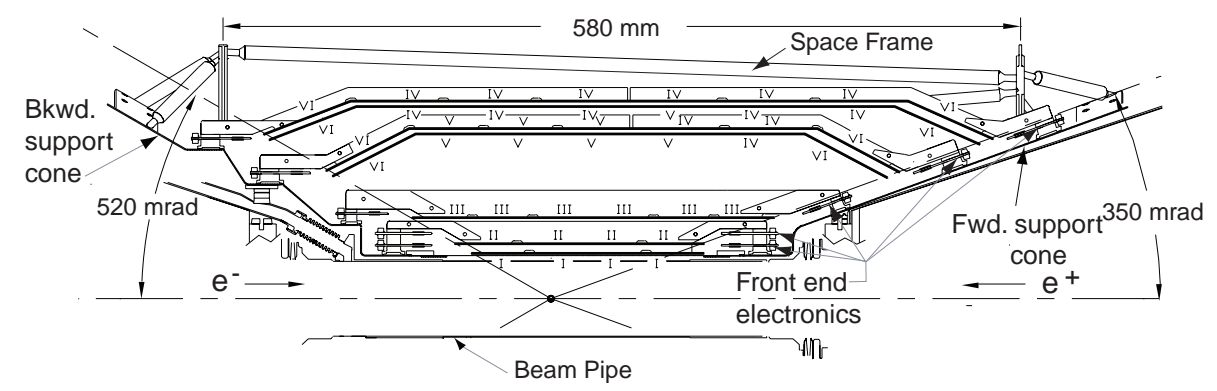

Figure 2.6: Longitudinal view of the SVT.

The two outer layers are positioned at larger radii, at $12 \mathrm{~cm}$ from the $z$-axis, to provide better position and angle information required to link tracks to the next layer of track detection in the drift chamber $(\mathrm{DCH})$.

Each layer is designed with a cylindrical shape, with the two outer-most layers having an arch shape design to maximize the solid angle coverage without using excess material. Figs. 2.6 and 2.7 show schematic views of the SVT. In total, $90 \%$ solid angle from the CM is covered by the SVT.

Charged tracks with momentum transverse to the beam line less than $100 \mathrm{MeV}$ will not reach the DCH so the SVT must provide stand-alone tracking information. Additionally, the SVT provides the most accurate measurement of angles of highmomentum tracks, which is required to achieve the design resolution in the Cherenkov angle detector, the DIRC, as discussed below.

\subsubsection{Drift Chamber}

The drift chamber provides the main tracking information for BABAR. It is located just outside the SVT, within the magnetic field and is designed to provide tracking information that is complementary to the precise vertexing information provided by the SVT. The DCH is also the only source of reconstruction information for any particle 


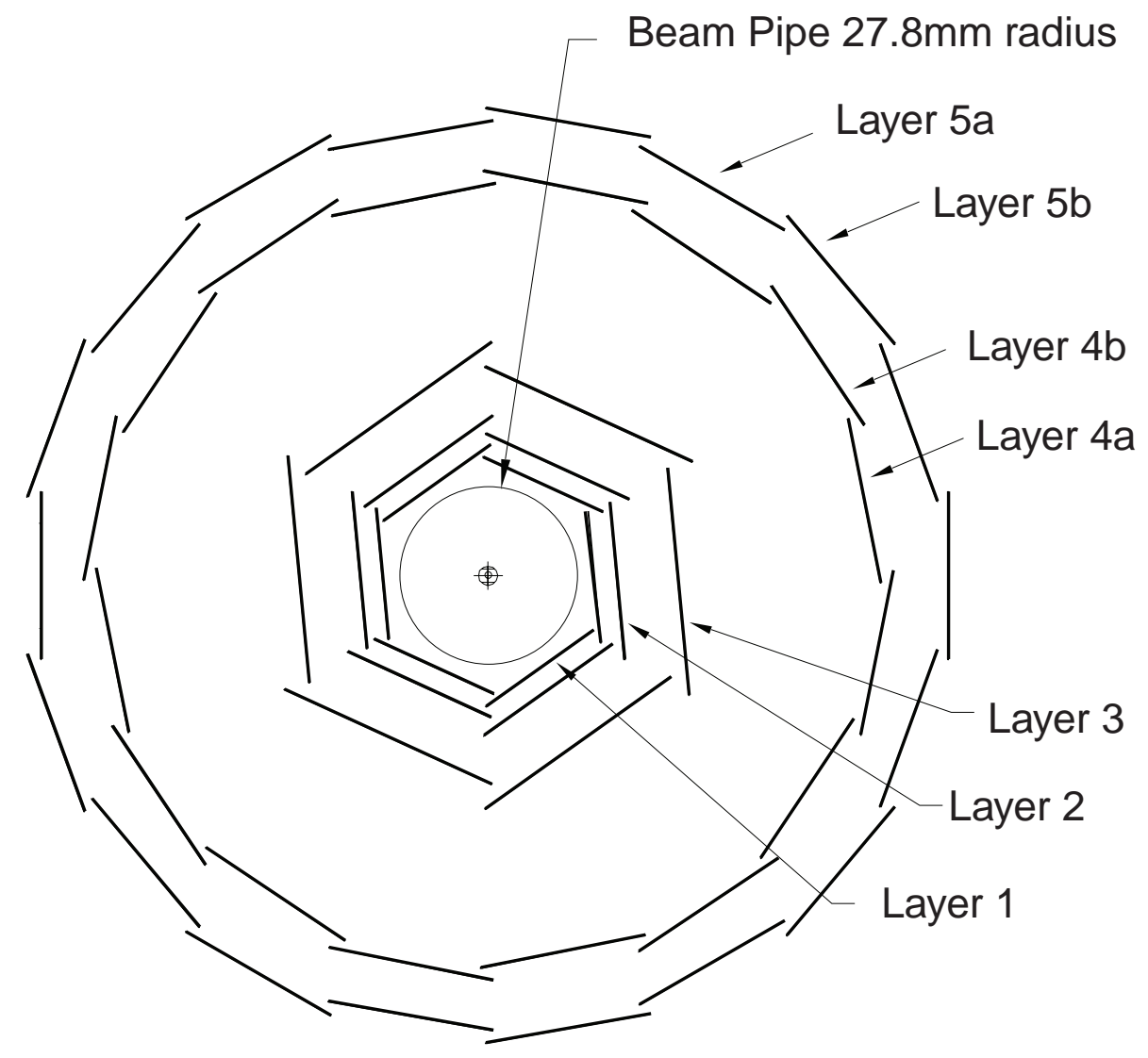

Figure 2.7: Transverse view of the SVT. 
that decays outside the SVT, such as many $K_{S}^{0}$ mesons. The transverse momentum resolution for the combined tracking system is $\sigma_{p_{T}} / p_{T}=0.0013 p_{T} \oplus 0.0045$, where the sum is in quadrature and $p_{T}$ is measured in $\mathrm{GeV}$.

The DCH also provides measurements of energy loss due to ionization $(\mathrm{d} E / \mathrm{d} x)$. For charged tracks with momenta of $700 \mathrm{MeV}$ or less, the $\mathrm{d} E / \mathrm{d} x$ measurements from the $\mathrm{DCH}$ provide the best $\mathrm{K}-\pi$ separation with a typical resolution of $7.5 \%$. For higher momentum tracks, the DIRC (described in Sec. 2.2.3) is more effective. In the extreme forward and backward regions the DCH provides the only source of particle indentification due to greater coverage. The DCH is also responsible for producing the signal needed for the charged-track trigger, one of the main trigger sources as discussed in Sec. 2.2.6.

The DCH is cylindrically shaped with an outer radius of $81 \mathrm{~cm}$ and a length of 2.8 m. It consists of 40 layers of hexagonal cells. Tracks with transverse momenta greater than $180 \mathrm{MeV}$ will pass through all 40 layers, each providing a position and $\mathrm{d} E / \mathrm{d} x$ measurement. The layers are grouped as sets of four into ten superlayers. Fig. 2.8 shows the four innermost such superlayers. The stereo angles of the superlayers are oriented slightly offset with respect to each other to allow for 3-dimensional positioning information. The pattern shown in Fig. 2.8 of an axial layer followed by a pair of stereo layers is repeated for all ten superlayers.

In total, 7104 drift cells make up the DCH. Each cell consists of one sense wire surrounded by six field wires, as shown in Fig. 2.9. A voltage of $1930 \mathrm{~V}$ is applied to the the sense wires, with the field wires held at ground. The cells are filled with a helium-isobutane gas mixture, where helium is chosen to reduce multiple scattering. As a charged particle passes through the cell, the gas is ionized and an avalanche gain of $\sim 5 \times 10^{4}$ is obtained by the time the signal reaches the sense wire to be read out. 


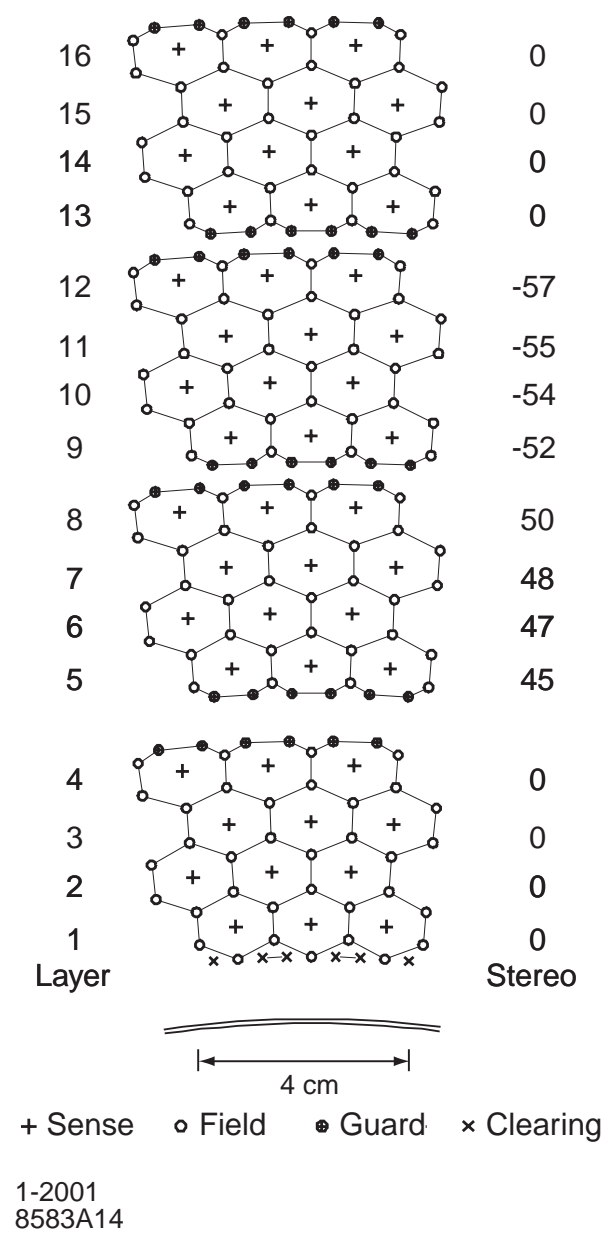

Figure 2.8: DCH drift cell configuration for the four innermost superlayers. The numbers on the right give the stereo angles in mrad of the sense wires in each layer. 


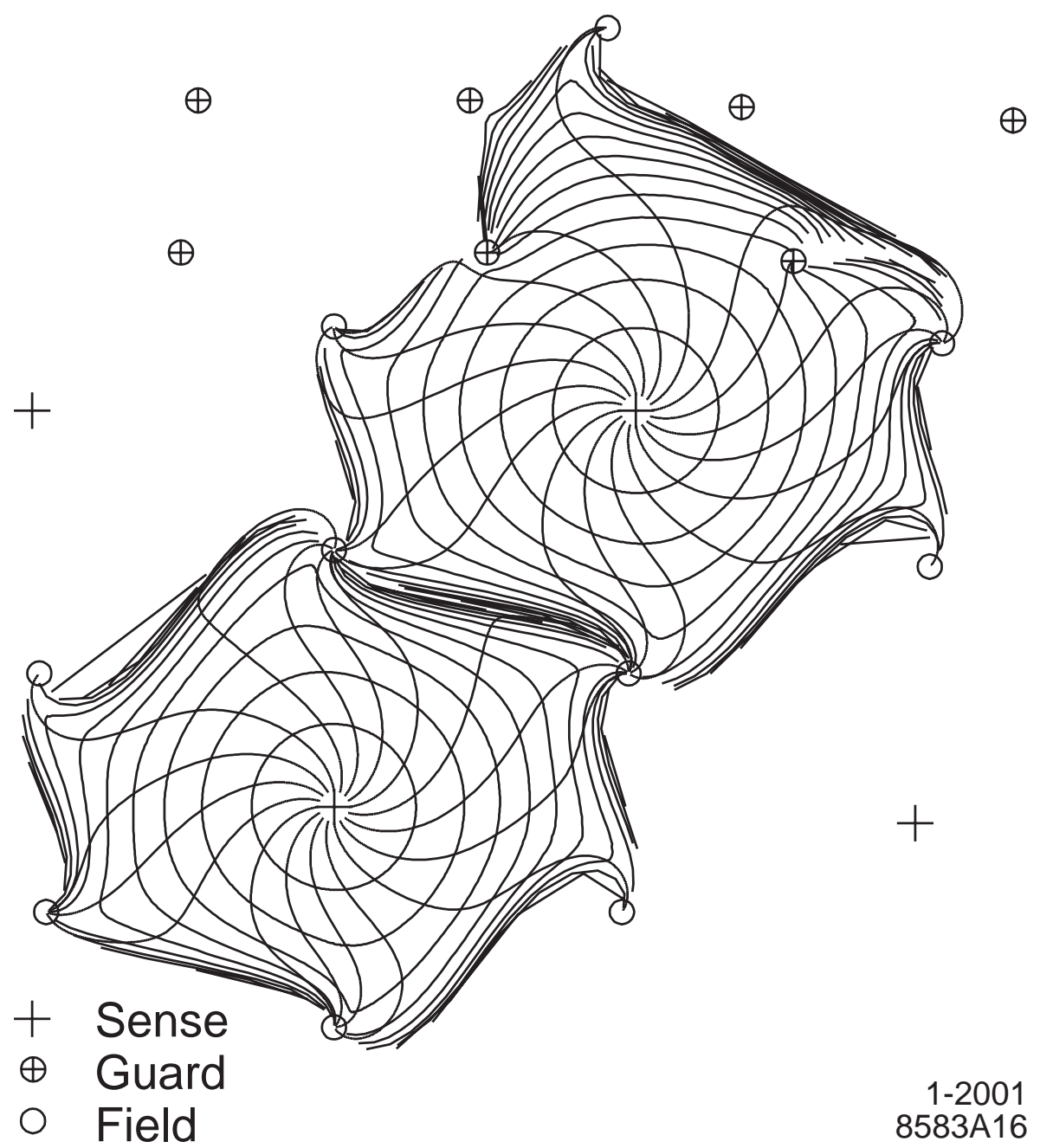

Figure 2.9: Two DCH drift cells, showing drift paths and $100 \mathrm{~ns}$ isochrones, or lines of constant drift time. Near the sense wires, the isochrones are circular, but the shape is distorted near the field wires. 


\subsubsection{DIRC}

The next concentric layer is a detector of internally reflected Cherenkov light (DIRC). The DIRC provides good particle identification (PID) for the experiment with particular focus on the identification of kaons and pions.

The detector consists of a layer of rectangular bars of silica. As charged particles with sufficient velocity to exceed the Cherenkov threshold pass through the silica a cone of Cherenkov light is emitted. The angle of this light is preserved through total internal reflection (TIR) within the silica bars, which have an index of refraction, $n=1.473$. This is shown schematically in Fig. 2.10. Each bar has a mirror placed perpendicular to the bar at the front end to reflect light toward the back end of the bar, where a series of photomultiplier tubes (PMT) detects the signals.

The Cherenkov light passes through a conical section filled with water called the Standoff Box before hitting the PMTs. A wedge of silica is placed at the backward end of each tube to provide a larger spread in the Cherenkov photons. This arrangement requires less precision in the position information from the PMTs to achieve the same accuracy. The wedge also serves to lessen the loss due to TIR at the silica/water transition.

The position and arrival time of detected photons are combined with the position information from the SVT and DCH to recreate an image of the track passing through the DIRC. The angle of the Cherenkov cone can be matched up with the track momentum information from the DCH to determine the mass of the particle that produced the track.

Kaons with momenta greater than $700 \mathrm{MeV}$ have sufficient velocity to produce Cherenkov light. Beyond this threshold the DIRC provides the best source of K- $\pi$ separation. The Cherenkov angle resolution of $2.4 \mathrm{mrad}$ provides $3 \sigma$ separation for tracks with a momentum of $3 \mathrm{GeV}$. Fig. 2.11 shows the PID performance of the DIRC, as well 


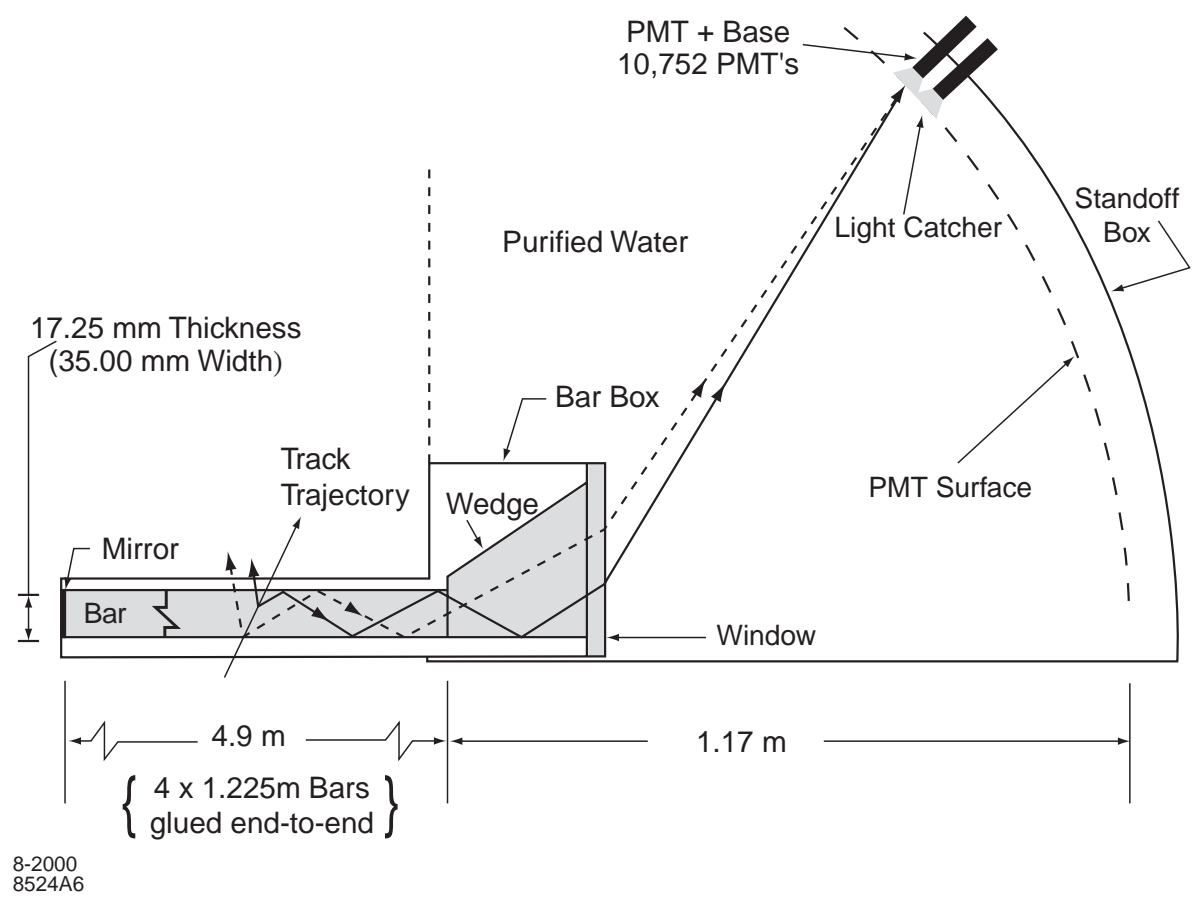

Figure 2.10: Schematic view of the DIRC silica bar and imaging PMTs. 
as that of the $\mathrm{d} E / \mathrm{d} x$ information from the DCH, which is complementary with optimal performance at momenta below the Cherenkov threshold. As momentum increases, the difference in angle between a pion and kaon decreases because the mass difference becomes less important and the PID resolution from the DIRC suffers.

The radial width of the DIRC is minimized to avoid degradation of the calorimeter resolution from interactions and minimize the volume needed for calorimeter material. The bars are only $17 \mathrm{~mm}$ thick (0.19 radiation lengths) as shown in the vertical-slice view in Fig. 2.12.

In addition to its use in the exclusive reconstruction of our decay modes, the good PID provided by the DIRC and $\mathrm{d} E / \mathrm{d} x$ is important in selecting the flavor of tag-side events with kaons for the time-dependent asymmetry measurements.

\subsubsection{Electro-Magnetic Calorimeter}

An electromagnetic calorimeter (EMC) is used to detect electromagnetic showers. It is required to detect showers over the energy range from $20 \mathrm{MeV}$ to $9 \mathrm{GeV}$. The low end of this limit is set by the need to detect low-energy $\pi^{0}$ 's. This is achieved with an energy resolution of $\sigma_{E} / E=\left\{2.3 / E(\mathrm{GeV})^{1 / 4} \oplus 1.9\right\} \%$. For $\pi^{0}$ 's above $2 \mathrm{GeV}$ the angular resolution becomes the limiting factor in the mass determination. An angular resolution of $\sigma_{\theta}=3.9^{\circ} \sqrt{E(\mathrm{GeV})}$ is obtained with a finely spaced array of crystals. Typical mass resolution for $\pi^{0}$ 's with momentum greater than $1 \mathrm{GeV}$ is $8 \mathrm{MeV}$. For high-energy photons, QED processes such as $e^{+} e^{-} \rightarrow e^{+} e^{-}(\gamma)$ (Bhabha) and $e^{+} e^{-} \rightarrow \gamma \gamma$ are required for use in calibration.

The detector consists of an array of thallium-doped cesium-iodide crystals built in two sections. A barrel-shaped array of tapered trapezoidal crystals circles the beam line; a block of crystals along the front end cap provides additional solid angle coverage for a total of $90 \%$ coverage in the CM. This is shown schematically in Fig. 2.13.

The crystals work by total absorption of the energy of entering particles. All 

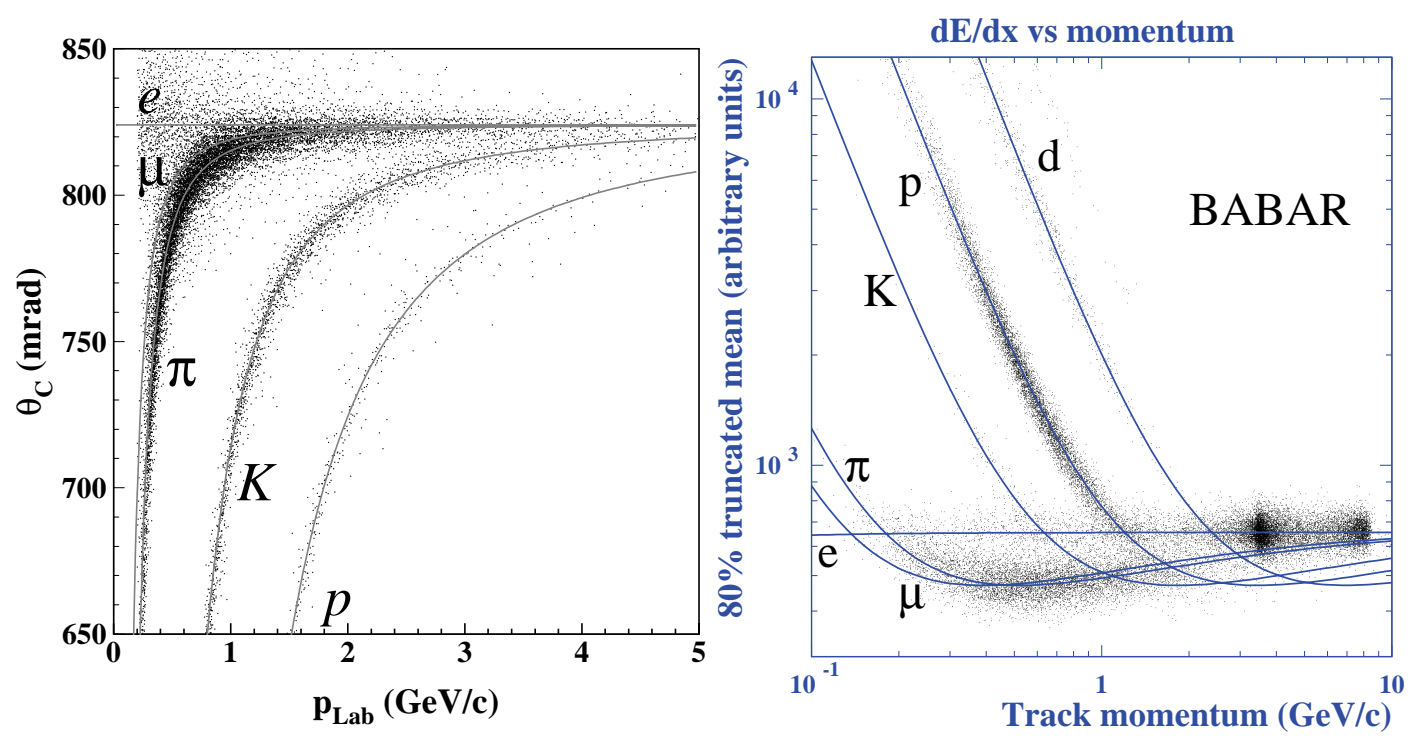

Figure 2.11: The fitted Cherenkov angle (left) of tracks from an inclusive sample of multi-hadron events plotted against the momentum of the tracks at the entrance to the DIRC bar box. The grey lines are the predicted values of the $\theta_{C}$ for the different particle species. Scatter plot (right) of $\mathrm{d} E / \mathrm{d} x$ versus momentum from beam scan data with parameterized Bethe-Bloch curves

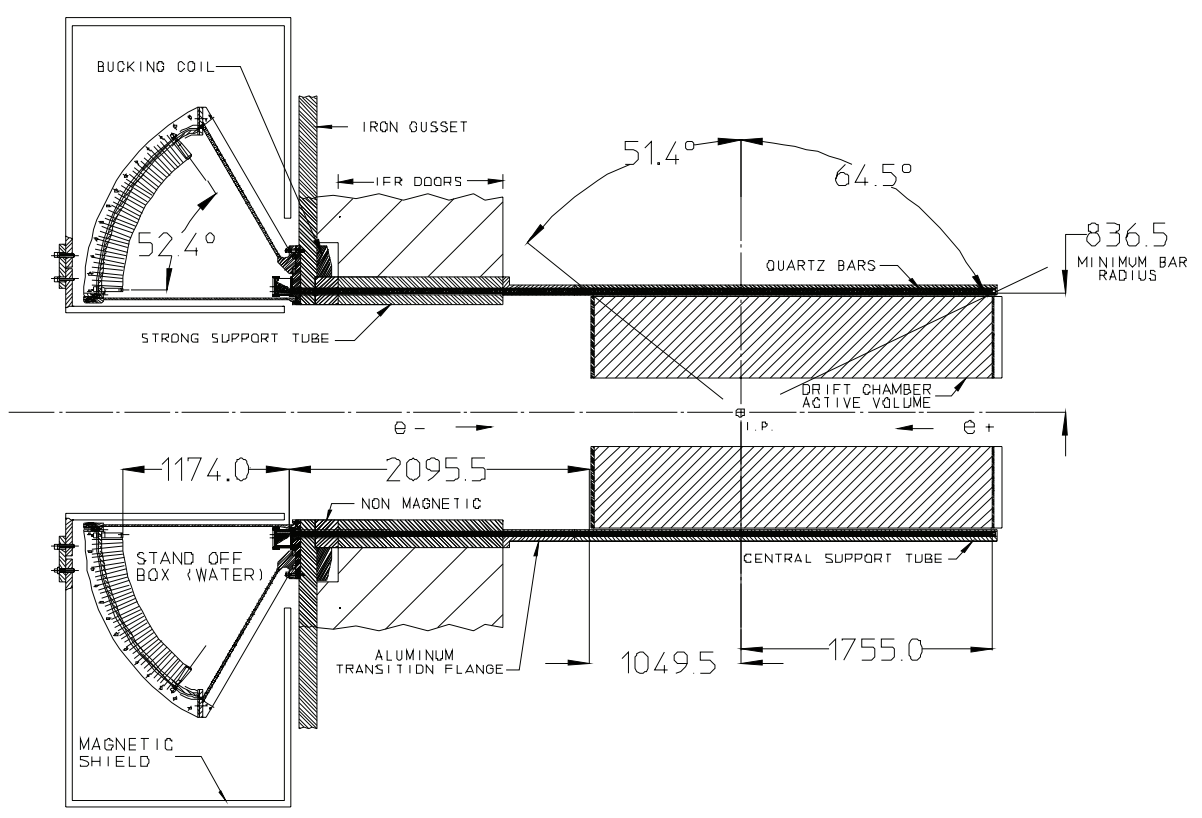

Figure 2.12: Vertical-slice view of the DIRC. 


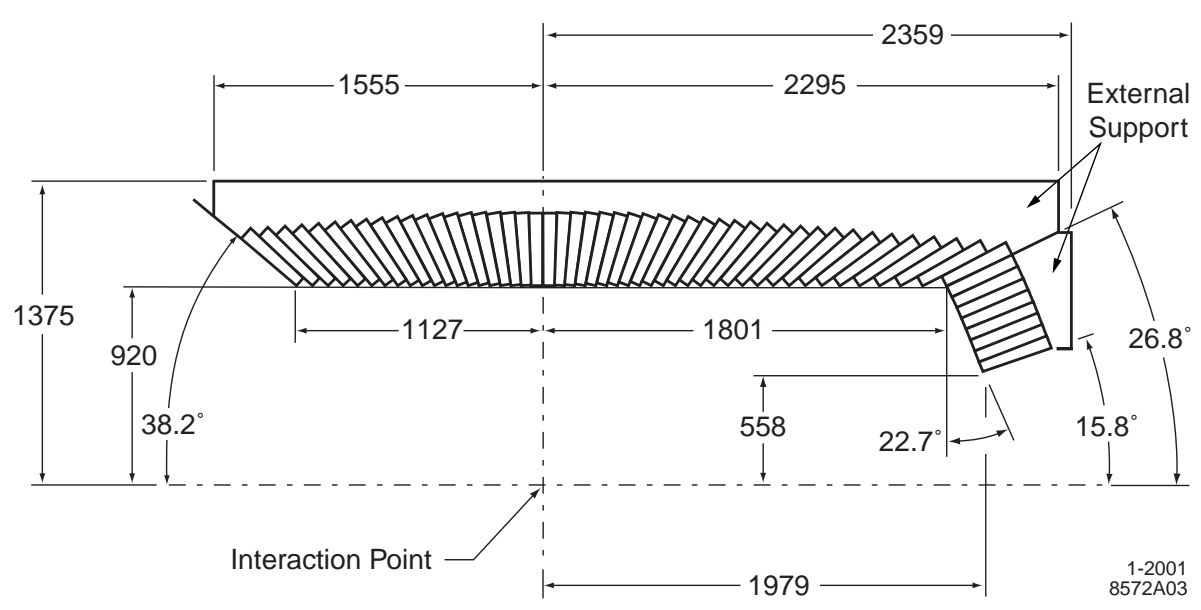

Figure 2.13: Longitudinal cross-section of the top half of the EMC.

the absorbed energy is channeled through the crystals to an electronic readout system at the back of each of the 6580 total crystals. The energy resolution is calibrated for low-energy photons by a $6.1 \mathrm{MeV}$ radioactive source and for high-energy photons by the known relationship between energy and polar angle from Bhabha events. In addition to $\pi^{0}$ 's, other neutral particles that decay to photons such as $\eta$ mesons are detected similarly. EMC energy deposits are also matched up with charged track information from the DCH to identify electrons used in flavor tagging.

\subsubsection{Instrumented Flux Return}

The outer-most subsystem is an Instrumented Flux Return (IFR) designed to identify muons and detect any remaining neutral hadrons, such as $K_{L}^{0}$ 's. The identification of charged leptons provides excellent flavor tagging information in the timedependent asymmetry measurements. The steel flux return of the superconducting magnet is used to fill gaps between 19 layers of detectors. The IFR consists of a barrel region and two end cap sections on the forward and backward ends as shown in Fig. 2.14. The IFR was originally instrumented with resistive plate chamber (RPC) detectors. With time, the initially good performance ( $~ 90 \%$ efficient for muons) of the 


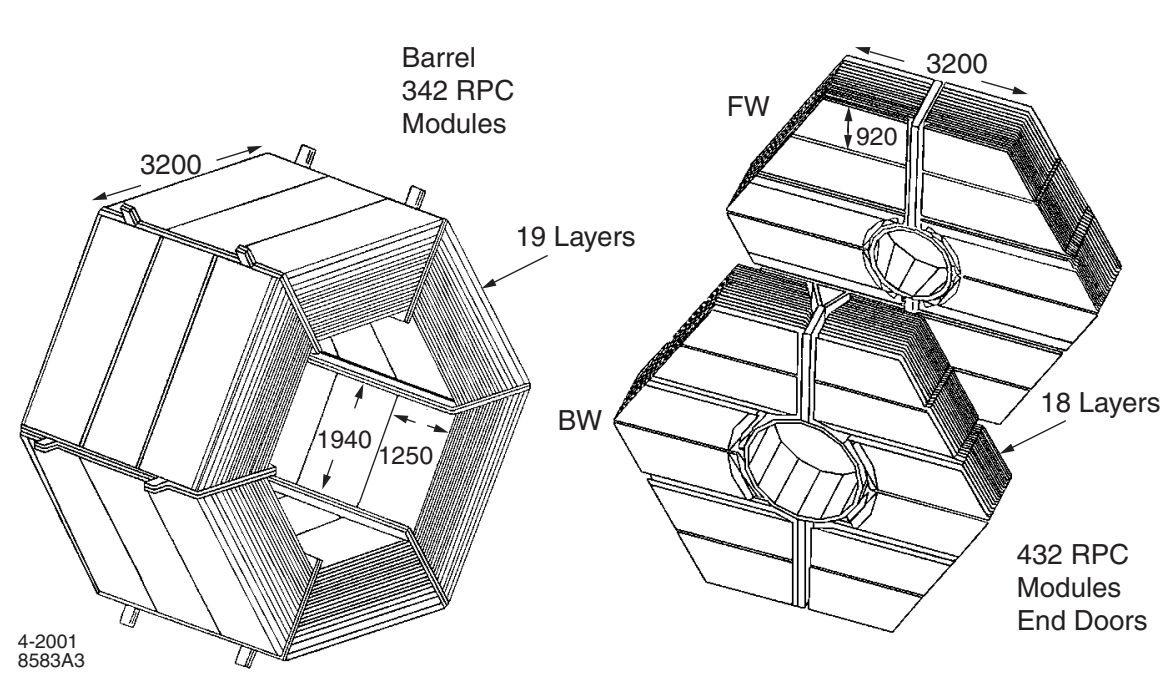

Figure 2.14: Barrel (left) and endcap (right) sections of the IFR.

RPCs started to degrade. In the summers of 2004 and 2006 the barrel region RPCs were replaced with Limited Streamer Tube (LST) detectors.

The LSTs consist of graphite coated PVC channels of cross-sectional area $2.5 \mathrm{~cm}^{2}$ with a wire running the length of the channel. When a voltage of $5 \mathrm{kV}$ is applied and the tube is held at ground, the chamber is at the edge of breakdown. A passing muon will ionize and start an avalanche of electrons moving toward the anode wire. This produces a signal on the wire that can be read on top of the high voltage. Strip detectors are also positioned perpendicular to the tubes to allow for a 2-D position readout. The steel plates from the flux return range in thickness from 2-10 cm and serve to filter out particles other than muons. With the upgrade to the LSTs, 6 of the layers of detectors were replaced by 2 -cm brass plates to provide additional absorption.

\subsubsection{Trigger}

The BABAR trigger is used to filter out background events from the $e^{+} e^{-}$collisions from those of potential physics interest. The collision data must be reduced to an amount manageable for storage and offline processing at a rate quick enough to 
record the data. Table 2.2 shows the cross-sections for the principle physics processes in $e^{+} e^{-}$collisions at $10.58 \mathrm{GeV}$. The trigger also must be able to filter out machine backgrounds.

To achieve this goal, a sequential two-level trigger is used. The Level 1 (L1) trigger is hardware based. The PEP-II beams have a bunch crossing spacing of $4.2 \mathrm{~ns}$. The L1 trigger must reduce this rate of $238 \mathrm{MHz}$ down to $2 \mathrm{kHz}$. This is accomplished by monitoring charged tracks in the $\mathrm{DCH}$ above a preset transverse momentum and the number and pattern of energy deposits in the EMC. Everything passing L1 is sent to the Level 3 (L3) trigger with L1 selecting $B \bar{B}$ events with $99.9 \%$ efficiency.

The L3 trigger reduces the rate below $100 \mathrm{~Hz}$. The online-processed software comprising the L3 reconstructs events and filters based on track and neutral cluster topologies. Only $\sim 13 \%$ of the L3 output is physics events, $40 \%$ is used for calibration and diagnostics, and the rest is unfiltered backgrounds. The total efficiency rate for $B \bar{B}$ pairs exceeds $99 \%$.

Table 2.2: Cross sections for the principal physics processes at $10.58 \mathrm{GeV}$. The $e^{+} e^{-}$ cross section refers to events with either the $e^{+}, e^{-}$, or both inside the EMC detection volume.

\begin{tabular}{lc}
\hline $\begin{array}{l}\text { Event } \\
\text { type }\end{array}$ & $\begin{array}{c}\text { Cross-section } \\
(\mathrm{nb})\end{array}$ \\
\hline$b \bar{b}$ & 1.1 \\
other $q \bar{q}$ & 3.4 \\
$e^{+} e^{-}$ & $\sim 53$ \\
$\mu^{+} \mu^{-}$ & 1.2 \\
$\tau^{+} \tau^{-}$ & 0.9 \\
\hline
\end{tabular}




\section{Chapter 3}

\section{Analysis Technique}

\subsection{Analysis Overview}

In this analysis $B$ meson decays are reconstructed in the channels $B^{+} \rightarrow \eta^{\prime} K^{+}$, $B^{0} \rightarrow \eta^{\prime} K^{0}, B^{+} \rightarrow \omega \pi^{+}, B^{+} \rightarrow \omega K^{+}$, and $B^{0} \rightarrow \omega K^{0}$. These are rare decays with branching fractions on the order of a few to a few tens per million $B$ decays. Measurement of such decays requires strict rejection of background events. The majority of our background events come from random combinations of particles that mimic our signal from continuum events. Further backgrounds arise from $B$ decays to other channels.

The process of rejecting this background begins with a skim that is applied to the data collected from the detector that loosely selects events that are likely to contain our signal. Next, reconstruction code is used to match combinations of tracks and neutral clusters into the composite particles in our decay channels such as the $\eta^{\prime}$ or $\omega$ mesons, and ultimately into $B$ meson candidates. A full reconstruction is used in these channels. After reconstruction a tighter set of selection criteria are applied to further reject background events.

The branching fractions, charge asymmetries, and time-dependent $C P$ asymmetries are measured using an unbinned maximum likelihood fit. Analyses at BABAR are done using a blind technique, which means that all selection criteria and experimental methods are decided upon before looking at the signal region of the data. This ensures that experimental bias is not allowed to shape selection criteria or fitting strategy. 


\subsection{Data, Monte Carlo Samples and Processing}

The analyses described here are based on data collected by the BABAR detector at the PEP-II asymmetric-energy $e^{+} e^{-}$collider from 1999-2006. The on-resonance

dataset consists of an integrated luminosity of $347 \mathrm{fb}^{-1}$ containing $383 \pm 4$ million $B \bar{B}$ pairs recorded at the $\Upsilon(4 S)$ resonance with a center of mass energy of $\sqrt{s}=10.58 \mathrm{GeV}$.

In addition to the data, we use GEANT4 Monte Carlo (MC) simulations [43] to better understand our signal decays and the various backgrounds to the signals. The MC simulates particle interactions in the detector as well as beam conditions and backgrounds. All of the same selection and reconstruction applied to data is applied to the MC. We start the processing from a skim BtoPP that applies loose selection criteria for events that contain a $B$ meson that decays to two high-energy charmless pseudoscalar or vector mesons. This skim selects events at a rate of $1.6 \%$ of the events passing the level 3 trigger.

For signal MC, we have 876,000 $\eta_{\rho \gamma}^{\prime} K^{0}$ events, 829,000 $\eta_{\eta \pi \pi}^{\prime} K^{0}$ events, 878,000 events for both $\eta^{\prime} K^{+}$modes, $777,000 \omega K_{S}^{0}$ events, and 164,000 for the $\omega K^{+}$and $\omega \pi^{+}$ modes. For $B \bar{B}$ background studies, we have used $341 \mathrm{M} B^{+} B^{-}$and $328 \mathrm{M} B^{0} \bar{B}^{0} \mathrm{MC}$ events as well as samples of 100,000-600,000 events for several dozen individual background modes. For the exclusive decay MC, only one $B$ decays in the specified channel, while the other $B$ decays generically.

\subsection{Reconstruction and Event Selection}

The event reconstruction and variable requirements are described in this section.

\subsubsection{Candidate Reconstruction}

$B$ candidates are formed by combining a candidate $\eta^{\prime}$ or $\omega$ meson with a charged $K, \pi$, or $K_{S}^{0}$ meson. The fits are done by fitting the final decay particles first and 
combining them into resonance candidates. These resonance candidates are then used to fit the next level up the decay chain until finally a $B$ meson candidate is constructed.

The fits are done using a vertexing and kinematic fit algorithm. Invariant masses, energy-momentum conservation and a common vertex for the composite decay point are used to combine the charged tracks and neutrals that comprise a composite candidate This fit determines values for the mass, momentum, and vertex position of the composite, which are used in the next level of fitting. For example, in the decay $B \rightarrow \omega K_{S}^{0}$ with $\omega \rightarrow \pi^{+} \pi^{-} \pi^{0}$, a fit for the $\pi^{0}$ is performed from two photons. Then two charged tracks are combined with the $\pi^{0}$ candidate to form an $\omega$ candidate. The internal degrees of freedom of the $\omega$ are then fixed and used in the fit for the $B^{0}$ candidate.

Additional constraints may also be applied in the fits, such as fixing the mass of the reconstructed candidate to a known value (mass constraint) or constraining the vertex to a known location such as that of the parent particle, or the beamspot (vertex constraint).

Primary charged tracks are taken from the GoodTracksLoose list. Requirements for this and other particle lists used in this analysis are given in App. A. In general, the names of the lists give a good indication of their content including the type of particle and the strictness of the selection.

$K_{S}^{0}$ mesons are reconstructed in the $\pi^{+} \pi^{-}$decay channel, selecting the tracks from the ChargedTracks list. The $K_{S}^{0}$ candidate is required to have a mass between 468 and $528 \mathrm{MeV}$ and the $K_{S}^{0}$ production point is constrained to the beam spot in the fit.

The $\eta^{\prime}$ mesons are reconstructed in two separate decay channels, $\eta^{\prime} \rightarrow \rho^{0} \gamma$ and $\eta^{\prime} \rightarrow \eta \pi^{+} \pi^{-}$. In the $\eta^{\prime} \rightarrow \rho^{0} \gamma$ decay channel the photon is required to have an energy greater than $100 \mathrm{MeV}$ and a lateral moment less than 0.8 . The $\rho^{0}$ candidate is constructed from two charged pions selected from the GoodTracksVeryLoose list with candidate mass between 440 and $1100 \mathrm{MeV}$. The $\eta^{\prime} \rightarrow \eta \pi^{+} \pi^{-}$decay channel is recon- 
structed using pions from the GoodTracksLoose list and an $\eta$ candidate reconstructed in the $\eta \rightarrow \gamma \gamma$ channel with vertex and mass constraints applied. The photons are required to have energy greater than $50 \mathrm{MeV}$ and lateral moment less than 0.8 . The $\eta$ candidate must have a mass between 470 and $620 \mathrm{MeV}$. Both $\eta^{\prime}$ decay channels are required to have an $\eta^{\prime}$ mass between 900 and $1010 \mathrm{MeV}$ and are mass constrained in the fit for the $B$.

The $\omega$ mesons are reconstructed in the $\omega \rightarrow \pi^{+} \pi^{-} \pi^{0}$ decay channel. The pion tracks are taken from the GoodTracksLoose list. The $\pi^{0}$ candidate is reconstructed from photon pairs with a minimum energy for each photon of $30 \mathrm{MeV}$, an energy greater than $200 \mathrm{MeV}$, and a mass between 100 and $160 \mathrm{MeV}$. The $\pi^{0}$ mass is constrained in the $\omega$ fit.

\subsubsection{Kinematic Variables}

A $B$ meson candidate is characterized by two kinematic variables, $\Delta E$ and $m_{\mathrm{ES}}$. These variables make use of the known kinematic information from the $\Upsilon(4 S)$ decay to $B \bar{B}$ pairs. $\Delta E$ is defined in a Lorentz-invariant way as,

$$
\Delta E=\left(2 q_{B} q_{0}-s\right) / 2 \sqrt{s}
$$

where $\sqrt{s}$ is the total CM energy of $e^{+} e^{-}$, and $q_{B}$ and $q_{0}$ are the 4-momenta of the $B$ candidate and the $e^{+} e^{-}$system. Intuitively, the variable is easier to understand when written in a different form,

$$
\Delta E=E_{B}^{*}-\frac{1}{2} \sqrt{s}
$$

where the ${ }^{*}$ signifies a quantity measured in the CM. $\Delta E$ then can be seen as the difference between the reconstructed energy of the $B$ candidate and it's expected value of half the CM energy. For true $B$ mesons, $\Delta E$ peaks at 0 . The beam energy substituted mass, $m_{\mathrm{ES}}$, is defined as,

$$
m_{\mathrm{ES}}=\sqrt{\left(\frac{1}{2} s+\mathbf{p}_{0} \cdot \mathbf{p}_{B}\right)^{2} / E_{0}^{2}-p_{B}^{2}}
$$


where $\left(E_{0}, \mathbf{p}_{0}\right)$ is the 4 -momentum of the $e^{+} e^{-}$system and $\mathbf{p}_{B}$ is the $B$ candidate momentum, both measured in the lab frame. This can also be written,

$$
m_{\mathrm{ES}}=\sqrt{\frac{s}{4}-p_{B}^{* 2}} .
$$

From this definition it is clear that $m_{\mathrm{ES}}$ is the $B$ candidate mass with half the energy of the beam substituted for the $B$ energy. This is equivalent to setting $\Delta E=0$. For true $B$ mesons, $m_{\mathrm{ES}}$ peaks at $m_{B}=5.28 \mathrm{GeV}$.

These variables are used because they are nearly independent and therefore reduce correlations in the fit. $\Delta E$ has a typical resolution of $25 \mathrm{MeV}$ in our signal modes, due mostly to detector resolution and $m_{\mathrm{ES}}$ has a typical resolution of $3 \mathrm{MeV}$ due to uncertainties in the beam energies.

At the reconstruction stage, loose cuts on the $B$ candidates are made with $|\Delta E|<$ $400 \mathrm{MeV}$ and the $B$ mass greater than $4.5 \mathrm{GeV}$.

\subsubsection{Event Shape Variables}

Since our signals are rare, most of the events selected are background. By far, the largest backgrounds come from continuum events, where no actual $B$ meson is produced. Since the $B$ mesons are produced nearly at threshold, in the center of mass they have a spherical topology. The $e^{+} e^{-} \rightarrow q \bar{q}$ continuum events with $q=u, d, s$, or $c$ produce energetic primary quarks and tend to have a jet-like topology.

To exploit this difference in topology between signal and continuum background, the shape variable $\left|\cos \theta_{\mathrm{T}}\right|$ is used. $\cos \theta_{\mathrm{T}}$ is defined as the cosine of the angle between the thrust axis of the $B$ candidate and the thrust axis of the rest of the event, calculated in the CM frame, with the thrust axis defined as the axis that maximizes the sum of the magnitudes of the longitudinal momenta of the particles. This variable has a nearly flat distribution for $B$ candidates while it is sharply peaked at \pm 1 for $q \bar{q}$ background events. Fig. 3.1 shows the distribution for signal $\mathrm{MC}$ and continuum data for a typical 
decay channel.

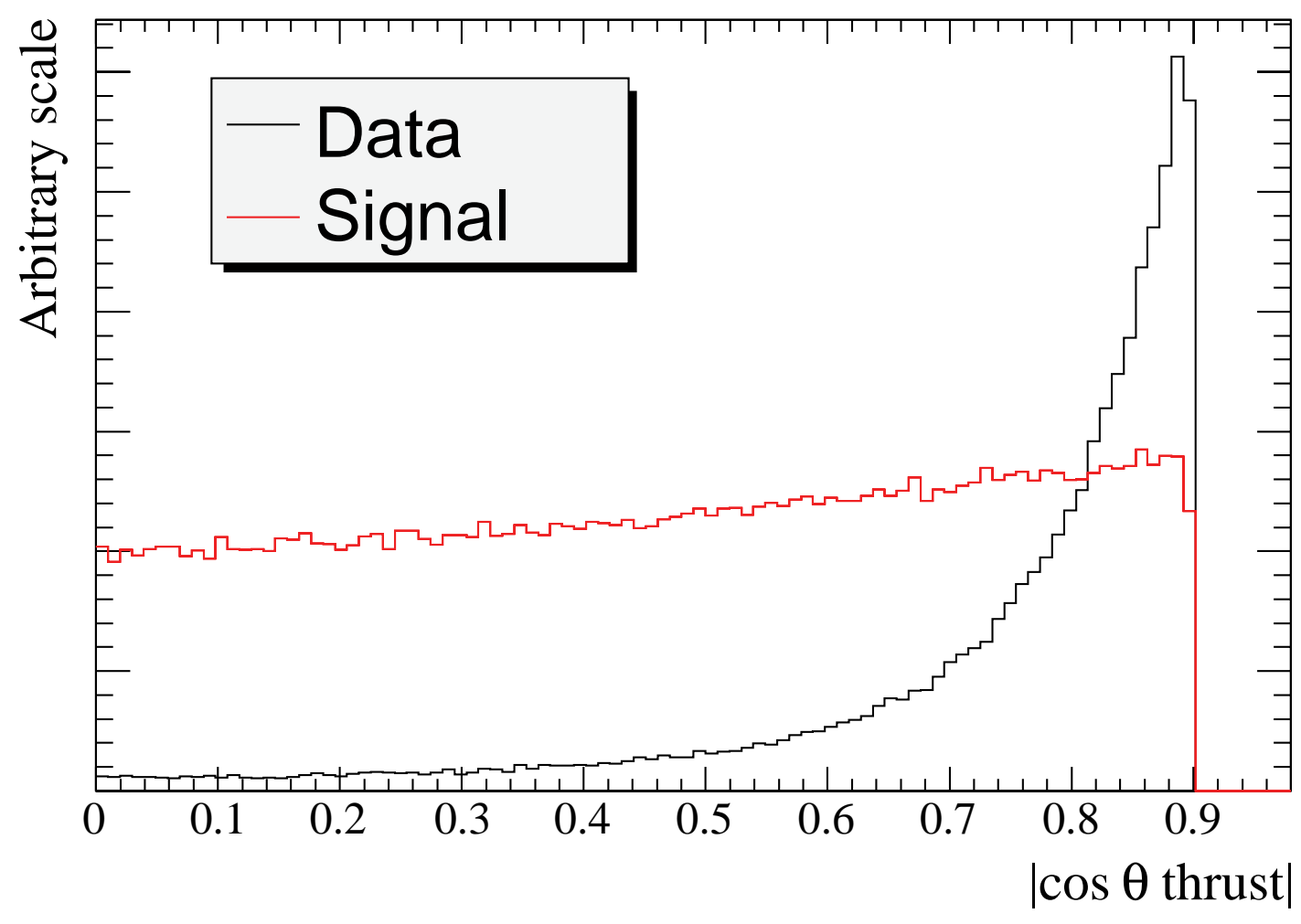

Figure 3.1: $\left|\cos \theta_{\mathrm{T}}\right|$ for signal MC (dotted) and $q \bar{q}$ background (solid) for the decay channel $B^{+} \rightarrow \eta_{\rho \gamma}^{\prime} K^{+}$with a cut applied at 0.9 .

In addition to cutting on the $\cos \theta_{\mathrm{T}}$ variable, further event shape information is used in the form of a Fisher discriminant $\mathcal{F}$, constructed from a linear combination of four variables: the absolute value of the cosine of the angle between the $B$ direction and the beam axis, the absolute value of the cosine of the angle between the thrust axis of the $B$ candidate and the beam axis, and the monomials $L_{0}$ and $L_{2}$, defined as,

$$
L_{n}=\sum_{i=R O E} p_{i} \times\left|\cos \left(\theta_{i}\right)\right|^{n}
$$

where the sum is over the tracks and neutrals in the rest of event, $p_{i}$ is the momentum of particle $i$, and $\theta_{i}$ is the angle between the direction of particle $i$ and the thrust axis of the $B$ candidate. We apply a shift to $\mathcal{F}$ based on tagging category information to 
remove the first-order correlation between the mean of the Fisher distribution and the tagging category. The tagging categories are discussed in Sec. 3.5.4.

\subsubsection{Final Selection Cuts}

After reconstruction, a final set of cuts is applied to determine the data sample that will be considered in the ML fit. These cuts are shown here.

- $N_{\text {tracks in event }} \geq N_{\text {tracks in decay mode }}+1$ (In order to be able to define a thrust vector for the rest of the event),

- $\left|\cos \theta_{T}\right| \leq 0.9$,

- $|\Delta E| \leq 0.2 \mathrm{GeV}$,

- $5.25 \leq m_{\mathrm{ES}}<5.2893 \mathrm{GeV}$,

- $-4<\mathcal{F}<5$, where $\mathcal{F}$ is the Fisher discriminant,

- $E_{\gamma}>50 \mathrm{MeV}$ (if not already tighter),

- the $\rho^{0}$ helicity $\mathcal{H}=\cos \theta_{H}$ (cosine of the vector meson's rest frame decay angle of a pion respect to $\eta^{\prime}$ flight direction) with $|\mathcal{H}|<0.9$;

- $E_{\pi^{0}}>250 \mathrm{MeV}$,

- $120<m_{\gamma \gamma}^{\pi^{0}}<150 \mathrm{MeV}$,

- $930<m_{\eta^{\prime}(\rho \gamma)}<980 \mathrm{MeV}$,

- $945<m_{\eta^{\prime}(\eta \pi \pi)}<970 \mathrm{MeV}$,

- $490<m_{\eta}<600 \mathrm{MeV}$,

- $470<m_{\rho}<980 \mathrm{MeV}$,

- $735<m_{\pi \pi \pi}^{\omega}<825 \mathrm{MeV}$, 
- $486<m_{\pi \pi}^{K_{S}}<510 \mathrm{MeV}$,

- $B$ vertex probability $>10^{-14}\left(\eta^{\prime}\right.$ modes $)$

- The $\omega$ and $\eta^{\prime}$ daughter charged tracks must not satisfy the Tight criteria of the electron selector, the VeryTight criteria of the proton selector, or the Tight criteria of the kaon selector. The selectors are based on likelihoods for each particle type calculated from the PID information from the DIRC and $\mathrm{d} E / \mathrm{d} x$ in the DCH. The efficiency of the selectors in Monte-Carlo data is corrected to match the response in real data.

- In the case where more than one candidate is found, the best one is selected based on the $\omega$ mass for the $\omega$ modes, the $\eta^{\prime}$ mass for the $\eta^{\prime} \rightarrow \rho^{0} \gamma$ modes and based on a $\chi^{2}$ constructed from the $\eta^{\prime}$ and $\eta$ masses for the $\eta^{\prime} \rightarrow \eta \pi^{+} \pi^{-}$modes.

For $B^{+} \rightarrow \eta^{\prime} K^{+}$we require:

- The DIRC pull for the kaon track hypothesis $<2$. DIRC pull is discussed further in Sec. 3.5.

For $B^{0} \rightarrow \eta^{\prime} K_{S}^{0}$ and $B^{0} \rightarrow \omega K_{S}^{0}$ we require the $K_{S}^{0}$ to satisfy:

- fit probability $>0.001$,

- flight length significance $\left(l / \sigma_{l}\right)>3$, where $l$ is the flight length and $\sigma_{l}$ is the uncertainty in the flight length.

For $B^{+} \rightarrow \omega K^{+}$and $B^{+} \rightarrow \omega \pi^{+}$, we require:

- $\left|\cos \theta_{T}\right| \leq 0.8$,

- Number of measured DIRC Cherenkov photons for the bachelor track $>5$,

- To distinguish between a high momentum $\pi$ or $K$, we make a joint fit to either $\omega K^{+}$, or $\omega \pi^{+}$and require the DIRC pull to lie within the range $[-3.5,+3.5]$. 
For the time-dependent analysis we also require:

- $|\Delta t|<20 \mathrm{ps,}$ where $\Delta t$ is the time difference between the decays of the $B \bar{B}$ pair and is described further in Sec. 3.5,

- $\sigma_{\Delta t}<2.5$ ps.

\subsection{Maximum Likelihood Fit}

This analysis utilizes an unbinned multivariate maximum likelihood (ML) fit. The fitting is performed using the RooRarFit fitting package [44]. RooRarFit is a general fitting package based on ROOT [45] and RooFit [46]. The requirements on the quantities used as input to the ML fit are loose to allow for high efficiency and to provide sufficient sidebands to characterize the background well.

\subsubsection{Fit Variables}

Depending on the channel, between three and six discriminating variables are used in the fit. Three variables are used in all fits: $m_{\mathrm{ES}}, \Delta E$, and $\mathcal{F}$. The $\omega$ modes also include the $\omega$ invariant mass $m_{\omega}$ and the $\omega$ helicity $\mathcal{H}_{\omega}$ (see Sec. 3.6.5).

The fit for $B^{+} \rightarrow \omega K^{+}$and $B^{+} \rightarrow \omega \pi^{+}$is done simultaneously. Because the final states differ only in the identification of the primary track, each can be a significant background for the other. This fit uses the PID information from the DIRC directly as a component in the fit as $S_{K, \pi}$, the pull of the DIRC Cherenkov angle from its central value for a kaon or a pion. More detail is given in Sec. 3.5.

Time-dependent fits are used to extract the time-dependent $C P$ asymmetry parameters, $S$ and $C$. In these fits, $\Delta t$ and the tagging category $c$ are used. More detail is given in Sec. 3.5. Fits without $\Delta t$ are used to extract branching fractions and charge asymmetries. 


\subsubsection{PDFs and the Likelihood}

For each species $j$ (signal, $q \bar{q}$ background, and $B \bar{B}$ background) and each category $c$, we define a probability density function (PDF) for event $i$ as the product of the PDFs for the separate discriminating variables:

$$
\mathcal{P}_{j, c}^{i}=\mathcal{P}_{j}\left(m_{\mathrm{ES}}{ }^{i}\right) \cdot \mathcal{P}_{j}\left(\Delta E^{i}\right) \cdot \mathcal{P}_{j}\left(\mathcal{F}^{i}\right) \cdot \mathcal{P}_{j}\left(m_{\omega}^{i}\right) \cdot \mathcal{P}_{j}\left(\mathcal{H}_{\omega}^{i}\right) \cdot \mathcal{P}_{j}\left(\Delta t^{i}, \sigma_{\Delta t}^{i}, c\right)
$$

For the $\eta^{\prime} \rightarrow \eta \pi^{+} \pi^{-}$channels, we do not include a $B \bar{B}$ component in the fit, as discussed in Sec. 3.9. For the charged modes, no $\Delta t$ PDF is used. For the $\eta^{\prime}$ modes, no $m_{\omega}$ or $\mathcal{H}_{\omega}$ PDF is used. For the $B^{+} \rightarrow \omega h^{+}$channels $\left(\omega \pi^{+}\right.$and $\left.\omega K^{+}\right)$, we include separate pion and kaon components and a $K$ - $\pi$-dependent $\Delta E$. Further details about the combined $K-\pi$ fitting method are given in Sec. 3.5.

The extended likelihood is constructed from these PDFs as

$$
\mathcal{L}=\prod_{c=0}^{6} \frac{\exp \left(-\sum_{j} N_{j, c}\right)}{N_{c} !} \prod_{i}^{N_{c}}\left(N_{s i g} f_{s i g, c} \mathcal{P}_{s i g, c}^{i}+N_{q \bar{q}, c} \mathcal{P}_{q \bar{q}}^{i}+N_{b \bar{b}} f_{s i g, c} \mathcal{P}_{b \bar{b}}^{i}\right)
$$

where the $N$ 's are the number of input events and the $f$ 's are the fraction in each tagging category.

For the charged modes, we also fit for the charge asymmetry by expressing the yield of $B^{ \pm}$events as $N_{ \pm}=N_{T}(1 \mp \mathcal{A}) / 2$. The total yield $N_{T}$ and $\mathcal{A}$ are free parameters in the fit.

\subsection{Joint $K-\pi$ Fit}

For the $B^{+} \rightarrow \omega h^{+}$modes we distinguish between direct kaon and pion charged tracks by their distributions in $\Delta E$ and $S_{K, \pi}$, the pull of the DIRC Cherenkov angle from its central value for kaons or pions.

Neglecting for this discussion the other discriminating observables, we define the 
PDF:

$$
\begin{aligned}
\mathcal{P}= & Y_{s}^{\pi} E_{s}\left(\Delta E^{\pi}\right) D\left(S^{\pi}\right)+Y_{s}^{K} E_{s}\left(\Delta E^{K}\right) D\left(S^{K}\right) \\
& +E_{b}\left(\Delta E^{\pi}\right)\left[Y_{b}^{\pi} D\left(S^{\pi}\right)+Y_{b}^{K} D\left(S^{K}\right)\right] \\
= & Y_{s}^{\pi} E_{s}\left(\Delta E^{\pi}\right) D\left(S^{\pi}\right)+Y_{s}^{K} E_{s}\left(\Delta E^{\pi}+\delta \Delta E\right) D\left(S^{\pi}-\delta S\right) \\
& +E_{b}\left(\Delta E^{\pi}\right)\left[Y_{b}^{\pi} D\left(S^{\pi}\right)+Y_{b}^{K} D\left(S^{\pi}-\delta S\right)\right],
\end{aligned}
$$

where for the second form we have introduced the new observables $\delta \Delta E=\Delta E^{K}-\Delta E^{\pi}$, the difference between $\Delta E$ evaluated under the assumption that the prompt charged track is a kaon and that evaluated with the pion assumption, and $\delta S=S^{\pi}-S^{K}$, the difference between the DIRC pull value for the two mass hypotheses.

The function $E_{s(b)}(\Delta E)$ represents the resolution density function for $\Delta E$ for signal (background) and $D(S)$ is the resolution function for measurement of $S$.

The observables $S^{\pi}, S^{K}$ are highly correlated through their dependence on the momentum, and likewise $\Delta E^{\pi}, \Delta E^{K}$. This is not a problem for the fit, but to facilitate the generation of toy $\mathrm{MC}$ and projection of the PDFs we need the transformation to the pairs $\left(S^{\pi}, \delta S\right)$ and $\left(\Delta E^{\pi}, \delta \Delta E\right)$ that distinguish the resolution functions from the separation functions. In these operations we obtain the separation functions from the data, while we model the resolution through the PDFs. The observables in these pairs are uncorrelated for pions, and for kaons have a known linear correlation that we implement explicitly in the PDF. We assume a common shape of the $E_{s}(\Delta E)$ function for pions and kaons. We evaluate the background $E_{b}\left(\Delta E^{\pi}\right)$ always with the pion hypothesis to avoid incompatible limits of integration. This leaves separate pion and kaon yields $Y_{s / b}^{\pi / K}$ for both signal and background.

The track momentum and polar angle dependence of the separation between pion and kaon are inserted into the fit by directing the integrator to obtain these distributions from the data. This procedure incorporates the correlations between $\Delta E, S$, and the $K-\pi$ separations in these quantities. 
sectionTime-dependent Fit

The time-dependent fits include the variable $\Delta t=t_{C P}-t_{\mathrm{tag}}$, the time difference between the decay of the signal $B$ meson and the $\operatorname{tag} B$ meson. Specific features of these fits are discussed in this section.

\subsubsection{The $B$ Vertices and $\Delta z$}

With the Lorentz-boosted CM, the $B$ mesons produced will fly far enough that the decay length is comparable to the experimental resolution. Assuming that the $B$ pair is produced exactly at threshold in $\Upsilon(4 S)$ decays, and that the boost is exactly along the $z$ axis, the decay position difference, $\Delta z \equiv z_{C P}-z_{\text {tag }}$, will be a two-sided exponential centered on zero. The exponential has an average size of $\langle|\Delta z|\rangle=\beta \gamma \tau_{B} \approx 250 \mu m$ and is a signed quantity since either of the $B$ mesons can decay first.

The fully reconstructed $B$ meson $\left(B_{C P}\right)$ vertex is obtained from a full fit to the decay chain with a generalized least squared method using iterative Lagrange multipliers. The $B$ vertex has a typical uncertainty of $\approx 50 \mu m$ on $z_{C P}$.

The tag-side $B$ vertex is more difficult to obtain. An inclusive approach is used to retain high efficiency. After the full reconstruction of $B_{C P}$, all remaining reconstructed charged tracks in the event are considered. Composite particles that decay to charged tracks outside the SVT ( $K_{S}^{0}$ 's and $\Lambda$ 's) are not used. Such composites decaying inside the SVT are combined into composite tracks and used with the other tracks to find the $B_{\text {tag }}$ vertex. Since $B_{C P}$ is fully reconstructed, its momentum can be used to point back to the beamspot ellipse in the $x-y$ plane to determine the $B$ pair production point. This information is used as an additional "pseudo-track" in the vertex fit for $B_{\text {tag. }}$ In total, the resolution on $z_{\operatorname{tag}}$ dominates the measurement of $\Delta z$, which has a typical uncertainty of $110 \mu \mathrm{m}$, or slightly better than half the flight length in the CM.

The assumptions above that give a pure exponential distribution of $\Delta z$ are not strictly true. The measurement of $\Delta z$ is complicated by several factors: the beam axis 
is tilted by $20 \mathrm{mrad}$ with respect to the $z$ axis; the beam energies are not constant giving the $\Upsilon(4 S)$ a Gaussian spread in momentum with a standard deviation of $6 \mathrm{MeV}$; and the $B$ mesons are not produced exactly at threshold giving them non-zero momentum in the $\Upsilon(4 S)$ frame. All of these effects, however, are quite small compared to the $\Delta z$ resolution. The impact and treatment of these assumptions is discussed in the following section.

\subsection{2 $\Delta z \rightarrow \Delta t$ Conversion}

Ultimately, a $\Delta t$ measurement is used in the fit, and the measured $\Delta z$ must be converted. This conversion is not trivial since the decays of the two $B$ mesons do not occur in a single reference frame and measuring the absolute decay time for each $B$ is not possible since no tracks originate at the production point of the $B$ pair. The basic approximation used is to calculate $\Delta t$ as $\Delta z /(\gamma \beta)$. A pair of additional refinements to improve the conversion are discussed below.

The decay vertex position is only measured along the $z$ direction with the position difference in $x$ and $y$ neglected. The displacement between the beam axis and the boost is accounted for by using only $(\beta \gamma)_{z}$, which reduces the full boost by $0.02 \%$. It is also possible to use kinematic information from the reconstructed $B$ mesons to improve on the assumption of production exactly at threshold. The momenta of the $B$ mesons are known as a function of the CM energy of the collision and the mass of the $B$. The $B$ mesons are back to back in the CM, but the exact $z$ boost for each $B$ depends on its direction with respect to $\hat{z}$. For the fully reconstructed $B$, this is known and can be combined with the first-order $\Delta t$ calculation to give an average correction to $\Delta t$ from the $B$ momentum of a factor of 1.002 .

In total, a typical uncertainty of $\sim 0.65 \mathrm{ps}$ is achieved in our decay modes, compared with the $B$ lifetime of 1.5 ps. 


\subsubsection{BReco Sample}

The $\Delta z$ resolution is found to be mostly independent of the signal $B$ decay mode since the resolution is dominated by the $z_{\text {tag }}$ resolution. Because the decay channels described here have relatively small signals, the $\Delta t$ shape parameters are taken from fits to data for a large, high purity control sample of $B \rightarrow D^{(*)} X$ events, where $X$ includes $\pi, \rho$, and $a_{1}$ mesons. This control sample is referred to as the BReco sample and is used widely throughout BABAR. Since the resolution varies depending on the particular decay angle of individual decay events, we optimize the statistical information in the sample by scaling the resolution by the $\Delta t$ uncertainty, $\sigma_{\Delta t}$. The quantity $\Delta t / \sigma_{\Delta t}$ is used in the fit.

\subsubsection{Flavor Tagging}

A time-dependent analysis depends on knowing the flavor of the reconstructed $B$ meson by tagging the flavor of its production partner from the $\Upsilon(4 S)$ decay. This analysis uses seven categories of tag identification based on the accuracy of the tagging mechanism. The categories range from semi-leptonic tag-side decays with a mistag rate of $3.0 \%$, through an untagged category where no tag information is obtained. The untagged category is not used in the determination of the $C P$ asymmetry parameters, but is included in the fit to better characterize the variable shapes.

In the case of imperfect tagging, Eq. 1.34 must be modified to include the mistag probabilities:

$$
\begin{aligned}
& f_{B^{0} \operatorname{tag}}=\left(1-w_{B^{0}}\right) f_{+}+w_{\bar{B}^{0}} f_{-}, \\
& f_{\bar{B}^{0} \operatorname{tag}}=\left(1-w_{\bar{B}^{0}}\right) f_{-}+w_{B^{0}} f_{+},
\end{aligned}
$$

where $w_{B^{0}}\left(w_{\bar{B}^{0}}\right)$ is the probability that a true $B^{0}\left(\bar{B}^{0}\right)$ meson is tagged as a $\bar{B}^{0}\left(B^{0}\right)$. Defining the dilutions, $D=\left(1-2 w_{B^{0}}\right)$ and $\bar{D}=\left(1-2 w_{\bar{B}^{0}}\right)$, the average dilution $\langle D\rangle$, 
and the dilution difference $\Delta D$,

$$
\begin{aligned}
\langle D\rangle & =\frac{D+\bar{D}}{2}, \\
\Delta D & =D-\bar{D},
\end{aligned}
$$

the mistag probabilities can be written as

$$
\begin{aligned}
& w_{B^{0}}=\frac{1-\langle D\rangle-\frac{\Delta D}{2}}{2}, \\
& w_{\bar{B}^{0}}=\frac{1-\langle D\rangle+\frac{\Delta D}{2}}{2} .
\end{aligned}
$$

This gives a mean mistag rate, $\langle w\rangle$, and mistag difference, $\Delta w$, defined by,

$$
\begin{aligned}
\langle w\rangle & =\frac{w_{B^{0}}+w_{\bar{B}^{0}}}{2}, \\
\Delta w & =\frac{w_{B^{0}}-w_{\bar{B}^{0}}}{2} .
\end{aligned}
$$

The decay rate distributions, assuming perfect vertex resolution, are then

$$
\begin{aligned}
& f_{B^{0} \mathrm{tag}}=\frac{e^{-|\Delta t| / \tau}}{4 \tau}\left[1+\frac{\Delta D}{2}+\langle D\rangle\left(S_{f} \sin \left(\Delta m_{d} \Delta t\right)-C_{f} \cos \left(\Delta m_{d} \Delta t\right)\right)\right] \\
& f_{\bar{B}^{0} \operatorname{tag}}=\frac{e^{-|\Delta t| / \tau}}{4 \tau}\left[1-\frac{\Delta D}{2}-\langle D\rangle\left(S_{f} \sin \left(\Delta m_{d} \Delta t\right)-C_{f} \cos \left(\Delta m_{d} \Delta t\right)\right)\right] .
\end{aligned}
$$

The final (observed) distribution $F(\Delta t)$ is the convolution of $f(\Delta t)$ with the signal vertex resolution function $\mathcal{R}_{\text {sig }}(\Delta t)$

$$
\begin{aligned}
& F_{B^{0} \mathrm{tag}}=f_{B^{0} \mathrm{tag}} \otimes \mathcal{R}_{\mathrm{sig}}, \\
& F_{\bar{B}^{0} \mathrm{tag}}=f_{\bar{B}^{0} \mathrm{tag}} \otimes \mathcal{R}_{\mathrm{sig}} .
\end{aligned}
$$

A total effective efficiency due to tagging is obtained by multiplying the fraction of events in each category by the dilution squared for that category and summing over the categories. The result is an effective tagging efficiency of $30.4 \pm 0.3 \%$. Table 3.1 shows the tagging categories and the mistag rate for each. These tagging parameters are obtained from fits to the BReco sample. Since the tag-side decay is independent of the signal decay the same tagging parameters are used for all decays. 
Table 3.1: Tagging categories, fraction of events in each category, $f_{\mathrm{tag}}$, and mistag rates, $\langle w\rangle$, obtained from the BReco sample.

\begin{tabular}{lrc}
\hline \hline Category & $f_{\text {tag }}(\%)$ & $\langle w\rangle(\%)$ \\
\hline Lepton & $8.7 \pm 0.1$ & $3.1 \pm 0.3$ \\
Kaon1 & $11.0 \pm 0.1$ & $5.2 \pm 0.4$ \\
Kaon2 & $17.2 \pm 0.1$ & $15.4 \pm 0.4$ \\
Kaon-Pion & $13.8 \pm 0.1$ & $23.5 \pm 0.5$ \\
Pions & $14.4 \pm 0.1$ & $32.9 \pm 0.5$ \\
Other & $9.6 \pm 0.1$ & $41.8 \pm 0.6$ \\
Untagged & $25.3 \pm 0.1$ & $50.0 \pm 50.0$ \\
\hline \hline
\end{tabular}

The fraction in each tagging category for signal, mistag fractions, mistag differences and background yields are all considered independently for each tagging category. The signal fractions, mistag fractions and mistag differences are fixed to the values found in the fit to BReco data. The full results of these fits are shown in App. B. The $B \bar{B}$ background tagging category fractions are also fixed to the values found in BReco.

\subsection{Probability Density Functions}

A multivariate maximum likelihood analysis makes use of probability density functions (PDFs) for the various input quantities in the fit. For each quantity a PDF must be determined for each of the components included in the fit. These components are signal, continuum, and, where necessary, $B \bar{B}$ background; see Sec. 3.9. These PDFs are determined with fits to the distributions for each of the variables for well identified samples of signal and background. The samples used for these fits are:

- signal MC

- on-peak data in sidebands chosen to avoid potential signal events

- signal MC from $B \bar{B}$ background modes 
Different samples are used for different quantities depending on the circumstances. The values of background PDF parameters obtained in these fits are used as initial values in the ML fits where they are floating. Appendix C shows PDF plots and correlations between input variables. In all cases, the best candidate is chosen before the PDFs are determined. The following sections give the shapes used for the PDFs for each ML fit variable.

\subsection{1 $\Delta E$}

We use a double Gaussian shape to parameterize the signal and charmless $B \bar{B}$ distributions. A control sample of reconstructed $B^{-} \rightarrow D^{0}\left(K^{-} \pi^{+} \pi^{0}\right) \pi^{-}$events is used to understand potential data/MC differences. This mode is chosen because of its high statistics in data and similar final-state topology to the decays studied here. This control sample shows that the MC is a reasonable representation of the data, but the core Gaussian width must be scaled by a factor of $1.05 \pm 0.05$, in order to have the appropriate width. For continuum background we use the on-peak data selected in a sideband defined by $m_{\mathrm{ES}}<5.27 \mathrm{GeV}$. The resulting distribution is well fit in all cases by a first or second degree polynomial.

For the joint $K-\pi$ fit for modes $B^{+} \rightarrow \omega K^{+}$and $B^{+} \rightarrow \omega \pi^{+}$, where the $\Delta E$ for both mass hypotheses is used in the fit, we require $0.026<\delta \Delta E<0.090$, where $\delta \Delta E \equiv \Delta E_{K}-\Delta E_{\pi}$. In practice this requirement does not remove any events from our sample.

\subsection{2 $m_{\mathrm{ES}}$}

We fit the $m_{\mathrm{ES}}$ distributions of signal MC events to double Gaussian functions. From the BReco control sample, we determine that the MC must be shifted to match the data. This is a time-dependent effect due to changing beam energies. The variable $m_{\mathrm{ES}}$ is constructed in such a way that for true $B$ decays, $m_{\mathrm{ES}}=m_{B}$. However, if the 
beam energies are mismeasured the value of $m_{\mathrm{ES}}$ will drift. We correct the data for this effect. For background we use the on-peak data in sidebands above and below the $\Delta E$ signal region $(100 \mathrm{MeV}<|\Delta E|<200 \mathrm{MeV})$ to obtain the parameters of an ARGUS function [47] defined by

$$
f(x) \propto x \sqrt{1-x^{2}} \exp \left[-\xi\left(1-x^{2}\right)\right]
$$

with $x \equiv 2 m_{\mathrm{ES}} / \sqrt{s}$. Variation of the beam energy means that it is not constant so the endpoint of the ARGUS distribution varies. After correcting $m_{\mathrm{ES}}$, we fit the distribution in data and find an endpoint of $5.2893 \mathrm{MeV}$ as shown in Fig. 3.2. We use this value in the fit. The $B \bar{B}$ component is fit with an ARGUS function and, where needed, an additional Gaussian.

Figure 3.2: Fit to $m_{\mathrm{ES}} q \bar{q}$ distribution for $B^{+} \rightarrow \eta_{\rho \gamma}^{\prime} K^{+}$with endpoint floating.

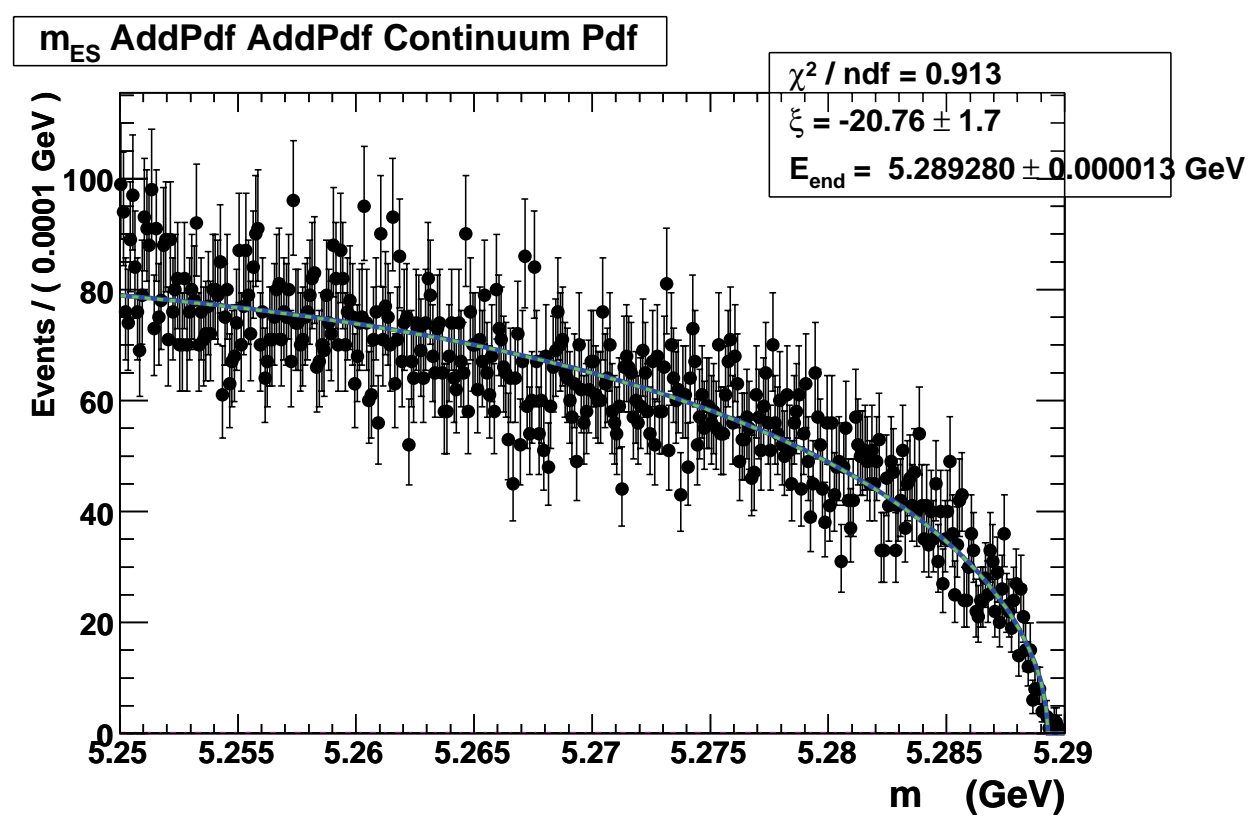

\subsubsection{Resonance Masses}

We obtain the PDFs for the $\eta^{\prime}$ and $\omega$ masses from signal MC samples. We considered using the $\eta^{\prime}$ mass as a variable in the ML fit, but find that its inclusion does 
not improve out fit uncertainties. For $\pi^{0}$ and $K_{S}^{0}$ we simply make cuts on the mass distributions and do not fit the mass spectra. This is because the candidates for these particles in the background are dominantly real, so the fit would serve only to deweight an already small combinatorial background. Signal resonance mass shapes are fit with a double Gaussian and the background shape is a first-order Chebyshev polynomial.

\subsubsection{Resolution Measurements}

We check that the resolution and central values for resonance mass peaks are correctly represented in the Monte Carlo by comparing with data. Where there are differences between Monte Carlo and data, we determine the appropriate values by which to shift the mean and scale the width of the peaking distribution. The samples of true $\eta^{\prime}$ and $\omega$ mesons in both the signal and the continuum background in the decay channels described here are large enough to determine potential data/MC differences and no control samples are needed. We determine the scale factor and shift parameter by allowing them to float in our on-peak data fits. We find that scale factors and shift values agree, within errors, across modes. To determine the best values to use in our analyses, we take the weighted average of the scale factors and shifts. In our final analyses we fix the shift and scale factors to the values listed in Table 3.2. Although we do not use the $\eta^{\prime}$ mass in our fits, we also calculate the shift and scale for this resonance. As resonances are typically fit with double Gaussians, we apply the shift to both Gaussian components, but scale only the width of the core Gaussian.

\subsubsection{Resonance Components in Background}

In addition to combinatorial background, there is a component of real resonance production that must be taken into account. We fit these distributions with the same functional form as the signal and a first degree Chebyshev polynomial for the combinatorial background. The fraction of all candidates which have a real resonance in the peak 
Table 3.2: Scale factors and shift parameters applied to $\Delta E$, and resonance mass distributions to correct for differences between data and Monte Carlo.

\begin{tabular}{lcc}
\hline \hline Variable & Scale Factor & Shift $(\mathrm{MeV})$ \\
\hline$\Delta E$ & $1.05 \pm 0.05$ & $0.0 \pm 2.0$ \\
\hline$\eta^{\prime}$ mass & & \\
$\eta \pi \pi$ & $1.05 \pm 0.05$ & $0.7 \pm 0.1$ \\
$\rho \gamma$ & $1.09 \pm 0.06$ & $1.7 \pm 0.3$ \\
\hline$\omega$ mass & $1.05 \pm 0.02$ & $1.1 \pm 0.1$ \\
\hline \hline
\end{tabular}

can be used as a fixed parameter in the ML fit or can be allowed to float (see Sec. 3.6.9), and is determined from a fit to the on-resonance side-band data. The parameters for the true resonance component are fixed to those found for the signal component, after application of the appropriate scale factors and offsets.

\subsubsection{Fisher Discriminant}

To determine the PDF for the Fisher discriminant $(\mathcal{F})$ we use signal MC for signal, a sideband of on-peak data $\left(m_{\mathrm{ES}}<5.27 \mathrm{GeV}\right)$ for $q \bar{q}$ background, and signal MC from significant crossfeed modes for the $B \bar{B}$ component. For signal, we fit with an asymmetric Gaussian, and add to this a second Gaussian for $q \bar{q}$ background. The asymmetric Gaussian, a Gaussian with different widths on the left and right sides of its peak, is implemented using three parameters, peak, width, and asymmetry, that have been constructed to reduce the very large correlation present between the parameters when constructed as a mean, and left and right width.

\subsubsection{Resonance Helicity}

In the case of pseudoscalar-vector (PV) decays of the $B$, the vector meson has a well known helicity distribution. For the $\omega$ decays, $\theta_{H}$ is defined as the angle between 
the normal to the $\omega$ decay plane (the plane of the 3 pions in the $\omega$ rest frame) and the candidate $B$ momentum, measured in the omega rest frame. The distribution is proportional to $\cos ^{2} \theta_{H}$ for a $B \rightarrow P V$ signal. For background, we expect $\mathcal{H} \equiv\left|\cos \theta_{H}\right|$ to have a nearly flat distribution. In all cases, the distributions are fit with Chebyshev polynomials.

\subsubsection{Correlations Among Discriminating Variables}

The likelihood function we use in the fits is based on the assumption that the variables used in the fit are uncorrelated. Appendix $\mathrm{C}$ shows the linear correlation coefficients for all pairs of variables used in the fit for Monte Carlo signal events and on-resonance data. The fact that these correlations are below $10 \%$ in all cases, except $m_{\omega}$ vs $\Delta E$ signal MC, justifies their exclusion from the fits. The effect of the $m_{\omega} v s \Delta E$ signal correlation (and all other signal correlations) is measured in the embedded toy studies (Sec. 3.10.1.2) and is shown to be small. A more detailed study of the correlations in App. D further supports the conclusion that the effect of correlations is small in these analyses.

\subsubsection{PID}

The PID PDF $S$ is a double Gaussian whose core component has a mean of 0.01, a width 0.96 and covers $96 \%$ of the PDF area, while the second Gaussian has a mean of -0.36 and a width of 2.3 . This shape is calibrated from $D^{*+} \rightarrow D^{0}\left(K^{-} \pi^{+}\right) \pi^{+}$ data events by the BABAR group studying the $B \rightarrow \pi K$ decays. Fig. 3.3 shows the distribution of $S_{\pi}$ for data, true pion signal and true kaon signal.

\subsection{8 $\Delta t$}

For the $C P$ sample we use the $C P$ model PDF convoluted with the resolution function described in Eq. 3.17. The resolution function $\mathcal{R}_{\text {sig }}(t)$ is the same triple Gaussian 


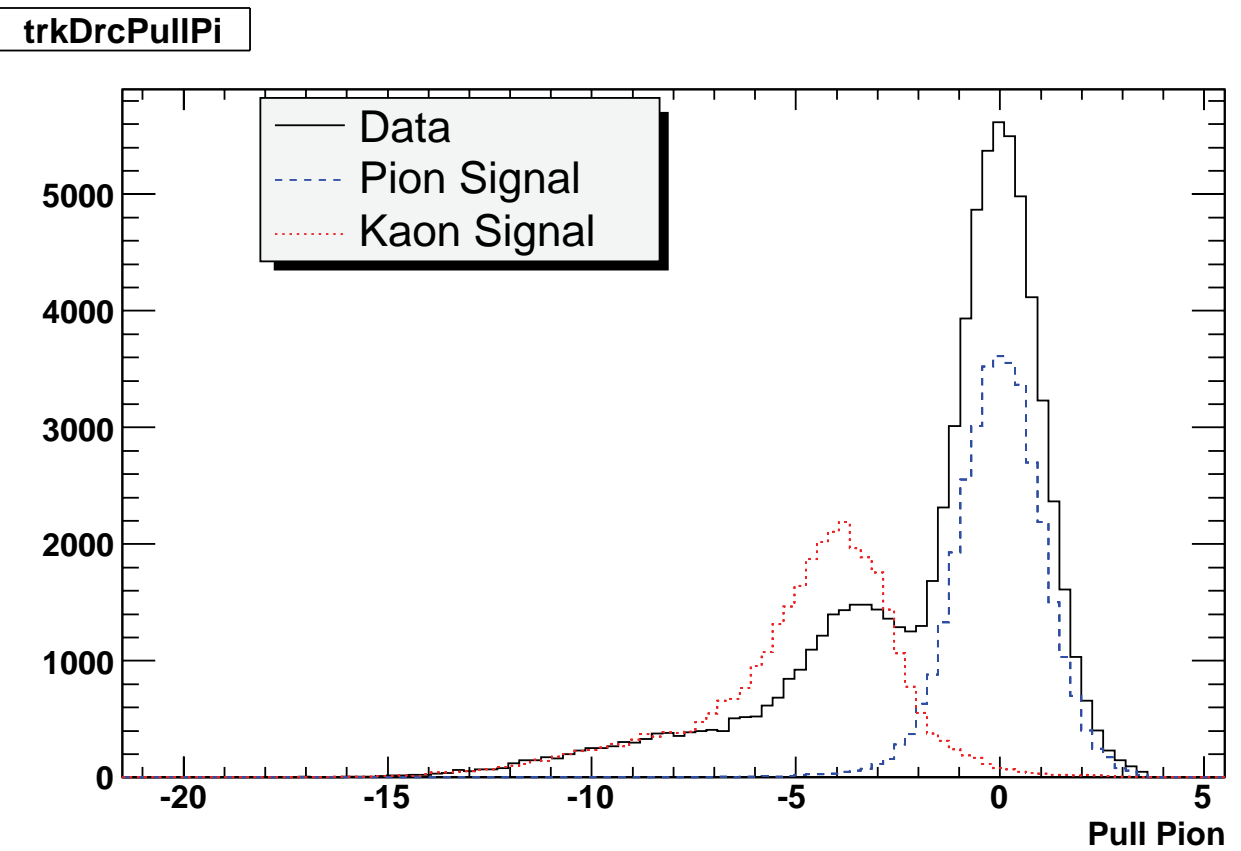

Figure 3.3: Pion pull distribution for $q \bar{q}$ background (solid), pion signal (dashed) and kaon signal (dotted) for $\omega h^{+}$. 
as used for BReco:

$$
\begin{aligned}
\mathcal{R}_{\text {sig }}(t) & =\left(1-f_{\text {tail }}-f_{\text {out }}\right) G\left(t, s_{\text {core }}^{\mu} \sigma_{t}, s_{\text {core }}^{\sigma} \sigma_{t}\right)+f_{\text {tail }} G\left(t, s_{\text {tail }}^{\mu} \sigma_{t}, s_{\text {tail }}^{\sigma} \sigma_{t}\right) \\
& +f_{\text {out }} G\left(t, \mu_{\text {out }}, \sigma_{\text {out }}\right)
\end{aligned}
$$

where $G\left(x, x_{0}, \sigma\right)$ is a Gaussian with bias $x_{0}$ and standard deviation $\sigma$.

The signal resolution parameters are taken from a fit to the BReco data. The core and tail means and widths are scaled (use $\Delta t / \sigma_{\Delta t}$ ), while only $\Delta t$ is used for the broad outlier component. The core Gaussian offset value is fit independently for the lepton tagging category and the non-lepton tagging categories. The tail Gaussian has

a fixed width of 3 , and the outlier Gaussian has a fixed mean of 0 and a width of 8 ps. The fractions and other means and widths are allowed to float. The results of the fit to BReco data are shown in App. B.

We fix $\Delta m_{d}$ and the $B$ lifetimes to the PDG values [2]: $\Delta m_{d}=0.507 \pm 0.005$ $\mathrm{ps}^{-1}, \tau_{B^{+}}=1.638 \pm 0.011 \mathrm{ps}$, and $\tau_{B^{0}}=1.530 \pm 0.009 \mathrm{ps}$ in the extraction of the $C P$ asymmetry parameters, $S$ and $C$.

The $q \bar{q}$ background $\Delta t$ distribution is modeled with on-peak sideband data. It is parameterized with the Gexp shape (a triple Gaussian convoluted with an exponential) though there are negligible changes in the results when a triple Gaussian is used instead. The background functions also use $\Delta t / \sigma_{\Delta t}$, consistent with signal.

\subsubsection{Floating Background Parameters}

Within the framework of RooFit we have the ability to float some of the PDF parameters in the maximum likelihood fit. By doing this, we include uncertainties in the values of these parameters in the fit statistical error and the background parameters are determined by making use of the larger statistics available in the full on-resonance sample rather than restricting the data to on-peak sidebands. We test with toy MC that our fitter can handle the number of degrees of freedom we use in our final fits as 
described in Sec. 3.10.

For the analyses described here, we float the most important parameters in the background (ARGUS exponent, $\Delta E$ slope, $\omega$ mass slope, real $\omega$ fraction and core $\mathcal{F}$ ) except in the case of $\eta^{\prime} \rightarrow \eta \pi^{+} \pi^{-}$modes where we fix the ARGUS exponent to the value found in the $\eta^{\prime} \rightarrow \rho^{0} \gamma$ fit because there are not enough background events to float the exponent in the fit. We fix the second Gaussian component of double Gaussian shapes to the value determined from signal Monte Carlo. Additionally, parameters to which our signal yields are insensitive such as tail components of peaking backgrounds are not floated. All floated parameters are initialized to the values determined from the $m_{\mathrm{ES}}$ or $\Delta E$ sideband. Specific listings of floated parameters, as well as their initial and final values, are given in Appendix $\mathrm{C}$.

\subsubsection{0 $\mathcal{H}_{\rho}$}

We considered including $\mathcal{H}_{\rho}$ as a variable in the fit for the $\eta^{\prime} \rightarrow \rho^{0} \gamma$ modes, but find the improvement in the errors on $S$ and $C$ to be $<1 \%$ and therefore decide not to use it.

\subsection{Efficiency, Corrections and Production Rate}

A raw (uncorrected) selection efficiency is determined from the ratio of the signal Monte Carlo events passing preselection and the total number of generated MC signal events. To obtain the final efficiency, the MC efficiency has to be corrected for an overestimate of the tracking and neutral efficiencies in the simulation.

The BABAR study of absolute tracking efficiency [48] reports that there is no tracking efficiency correction necessary with a systematic error of $0.5 \%$ per track for GoodTracksLoose and $0.4 \%$ per track for GoodTracksVeryLoose. The study is done comparing data and $\mathrm{MC}$ in tau-pair events where one of the taus decays with three tracks and the other with a single high-energy electron track to identify the event as a 
tau pair.

The Neutral Identification and Reconstruction working group gives a recipe [49] for correcting the neutral reconstruction efficiency and smearing the Monte Carlo to make the photon energy resolution match the data in $\pi^{0}$ reconstruction. The study is done using tau-pair events comparing the efficiency in data and MC for $\tau \rightarrow X \rho^{+}$and $\tau \rightarrow X \pi^{+}$decays. From this study, we apply a correction of $0.968 \pm 0.030$ per secondary neutral $\pi^{0}$ from an $\omega$ resonance. While no specific study has been done for $\eta$ decays to $\gamma \gamma$, the momentum dependence of the $\pi^{0}$ correction is mostly flat across the relevant range. Therefore, we use the $\pi^{0}$ value which most closely matches our $\eta$ reconstruction, that of "pi0Loose," and apply a correction of $0.983 \pm 0.030$. For the single photon in $\eta^{\prime} \rightarrow \rho \gamma$ decays we follow the neutrals group recipe in making no correction with an uncertainty of 0.018 .

We have determined the $K_{S}^{0}$ efficiency correction and associated systematic errors following the recipe described in [50] and apply a correction of $0.993 \pm 0.014$ for the $K_{S}^{0}$ in the $\eta^{\prime}$ modes and a correction of $0.991 \pm 0.015$ for $\omega K_{S}^{0}$. The study is done using inclusive $K_{S}^{0}$ samples in data and MC to compare the vertex-displacement dependence on efficiency for our event-shape topology.

The PID selectors are used to veto tracks of unwanted species. We therefore also apply (in code) the PID selector corrections provided by the PID group [51]. The vetos require DIRC, EMC and IFR signatures that are inconsistent with tracks being electrons or muons.

Finally, the efficiency must be corrected for resonance branching fractions since the channel of interest is forced in signal MC. The relevant efficiency information is summarized in the results tables (Sec. 4.1).

The luminosity is calculated using $\mu$ pair and other QED processes as described in Sec. 2.1. The number of $B \bar{B}$ pairs produced is calculated as a comparison of the ratio of hadronic events to $e^{+} e^{-} \rightarrow \mu^{+} \mu^{-}$events for the on- and off-resonance samples, 
with the assumption that the difference is fully attributable to $\Upsilon(4 S)$ production. Equal numbers of charged and neutral $B$ mesons are assumed.

\subsection{Two-track Backgrounds}

Bhabha scattering, muon-pair and tau-pair production, and two-photon processes are all common. Each is characterized by low charged track multiplicity of two or fewer. MC simulations show that the minimum charged track requirement made in event selection selection (at least $>4$ ) is effective in reducing these background to negligible levels.

\section{9 $\quad B \bar{B}$ Backgrounds}

While not as large as the background from continuum, background from real $B$ events can appear signal-like and must be accounted for. The full analysis selection is applied to a generic $B \bar{B}$ MC sample. Events from $b \rightarrow c$ processes are removed from the sample because they tend to appear continuum-like in the fit variables, and have been shown not to cause a bias in our signal. The more troublesome events are the charmless backgrounds. The preliminary study of generic MC provides a list of specific decay channels for further study. MC samples of 100,000-600,000 events for each significant $B \bar{B}$ background are generated. The full selection procedure is run for each channel and the events that satisfy the criteria are mixed in appropriate proportions based on selection efficiency and branching fraction.

For the $\eta^{\prime}$ modes, we find expected $B \bar{B}$ backgrounds of only 16 and 4 events entering the fit for $B^{+} \rightarrow \eta_{\eta \pi \pi}^{\prime} K^{+}$and $B^{0} \rightarrow \eta_{\eta \pi \pi}^{\prime} K^{0}$, for which we apply a systematic. For the $\eta^{\prime} \rightarrow \rho^{0} \gamma$ channels we apply the full procedure outlined above. The results of this study can be seen Table 3.3 for the $B^{+} \rightarrow \eta_{\rho \gamma}^{\prime} K^{+}$decay and in Table 3.4 for the $B^{0} \rightarrow \eta_{\rho \gamma}^{\prime} K^{0}$ decay. A single $B \bar{B}$ background component is added to the fit to properly account for these small backgrounds. 
Table 3.3: Potential $B \bar{B}$ background for the $B^{+} \rightarrow \eta_{\rho \gamma}^{\prime} K^{+}$mode from exclusive charmless $B$ decays included in the $B \bar{B}$ background component. We show efficiency for the mode to pass selection cuts, the measured or estimated branching fraction, the appropriate product branching fraction given how the MC was produced, the estimated background normalized to 384 million $B \bar{B}$ events and the number of events we include in the file we use for making PDFs. An $*$ denotes an estimated branching fraction.

\begin{tabular}{|c|c|c|c|c|c|c|}
\hline Bkg. channel & Mode \# & $\begin{array}{c}\mathrm{MC} \epsilon \\
(\%)\end{array}$ & $\begin{array}{l}\text { Est. } \mathcal{B} \\
\left(10^{-6}\right)\end{array}$ & $\prod \mathcal{B}_{i}$ & $\begin{array}{l}\text { Norm. \# } \\
B \bar{B} \text { Bkg. }\end{array}$ & $\begin{array}{l}\text { \# in PDF } \\
\text { Bkg. file }\end{array}$ \\
\hline$B^{+} \rightarrow K^{+} \pi^{-} \pi^{+}($Dalitz $)$ & 6846 & 1.7 & $54.8_{-2.9}^{+2.9}$ & 1.000 & 357.4 & 6236 \\
\hline$B^{0} \rightarrow a_{1}^{-}\left(\rho^{0} \pi^{-}\right) K^{+}$ & 4871 & 2.28 & $17^{*}$ & 0.500 & 74.4 & 1298 \\
\hline$B^{+} \rightarrow a_{1}^{0} K^{+}$ & 4874 & 2.08 & $9^{*}$ & 1.000 & 72 & 1256 \\
\hline$B^{0} \rightarrow \rho^{+} K^{-}$ & 1044 & 1.54 & $9.9_{-1.5}^{+1.6}$ & 1.000 & 58.5 & 1019 \\
\hline$B^{+} \rightarrow \phi_{3 \pi} K^{+}$ & 2713 & 6.5 & $8.3_{-0.65}^{+0.65}$ & 0.155 & 32.1 & 560 \\
\hline$B^{+} \rightarrow \rho K^{*}(1430)$ & 6102 & 0.15 & $40^{*}$ & 1.000 & 23.7 & 413 \\
\hline$B^{+} \rightarrow K^{+} K^{-} K^{+}$( Dalitz) & 6845 & 0.11 & $33.7_{-1.5}^{+1.5}$ & 1.000 & 14.8 & 258 \\
\hline$B^{+} \rightarrow \omega K^{+}$ & 1250 & 0.62 & $6.7_{-0.6}^{+1.5}$ & 0.891 & 14.3 & 249 \\
\hline$B^{0} \rightarrow a_{1}^{+}\left(\rho^{0} \pi^{+}\right) \pi^{-}$ & 1012 & 0.17 & $39.7_{-3.7}^{+0.6}$ & 0.500 & 12.8 & 223 \\
\hline$B^{0} \rightarrow a_{1}^{-}\left(\rho^{-} \pi^{0}\right) K^{+}$ & 4960 & 0.44 & $15^{*}$ & 0.500 & 12.6 & 220 \\
\hline$B^{+} \rightarrow \rho^{0} \pi^{+}$ & 1220 & 0.36 & $8.7_{-1.1}^{+1.0}$ & 1.000 & 11.9 & 208 \\
\hline$B^{0} \rightarrow \rho K^{*}(1430)$ & 6104 & 0.15 & $20^{*}$ & 1.000 & 11.8 & 205 \\
\hline$B^{0} \rightarrow \pi^{-} K_{0}^{*+}(1430)_{K^{+} \pi^{0}}$ & 4697 & 0.19 & $46.6_{-6.6}^{+5.6}$ & 0.310 & 10.5 & 182 \\
\hline$B^{+} \rightarrow a_{1}^{0} \pi^{+}$ & 4156 & 0.13 & $20^{*}$ & 1.000 & 10 & 174 \\
\hline$B^{0} \rightarrow K^{+} \pi^{-}$ & 1028 & 0.12 & $18.9_{-0.7}^{+0.7}$ & 1.000 & 8.6 & 150 \\
\hline$B^{+} \rightarrow \rho^{0} K_{K^{+} \pi^{0}}^{*+}\left(L, f_{L}=1\right)$ & 2355 & 1.83 & $3.6_{-1.8}^{+0.9}$ & 0.333 & 8.4 & 147 \\
\hline$B^{0} \rightarrow \eta_{\rho \gamma}^{\prime} K_{K^{+} \pi^{-}}^{* 0}$ & 2268 & 2.68 & $3.8_{-1.2}^{+1.2}$ & 0.197 & 7.7 & 134 \\
\hline$B^{+} \rightarrow \eta_{\eta \pi \pi}^{\prime} K^{+}$ & 1506 & 0.15 & $69.7_{-2.7}^{+2.8}$ & 0.174 & 7.2 & 125 \\
\hline$B^{+} \rightarrow \rho^{+} \rho^{0}\left(L, f_{L}=0.96\right)$ & 2390 & 0.1 & $18.3_{-3.4}^{+3.4}$ & 0.96 & 6.5 & 114 \\
\hline$B^{+} \rightarrow \eta_{\rho \gamma}^{\prime} \pi^{+}$ & 1509 & 2.16 & $2.6_{-0.5}^{+0.4}$ & 0.295 & 6.4 & 110 \\
\hline$B^{0} \rightarrow K_{K^{+} \pi^{0}}^{*+} \pi^{-}$ & 1226 & 0.48 & $9.8_{-1.1}^{+1.5}$ & 0.333 & 6 & 104 \\
\hline$B^{+} \rightarrow \eta_{\rho \gamma}^{\prime} K_{K^{+} \pi^{0}}^{*+}$ & 2773 & 2.98 & $4.9_{-1.9}^{+2.1}$ & 0.098 & 5.5 & 95 \\
\hline$B^{+} \rightarrow \rho^{+} K_{K^{+} \pi^{-}}^{* 0}\left(L, f_{L}=0.5\right)$ & 2244 & 0.35 & $4.65_{-0.85}^{+0.85}$ & 0.666 & 4.2 & 72 \\
\hline$B^{0} \rightarrow \rho^{0} K_{K^{+} \pi^{-}}^{* 0}\left(L, f_{L}=0.5\right)$ & 2359 & 1.7 & $0.65_{-0.65}^{+0.05}$ & 0.667 & 2.8 & 49 \\
\hline$B^{+} \rightarrow \pi^{+} \pi^{+} \pi^{-}$(N.R.) & 1230 & 0.23 & $\begin{array}{l}-0.00 \\
3_{-3.0}^{+3.0}\end{array}$ & 1.000 & 2.7 & 46 \\
\hline$B^{0} \rightarrow \rho^{+} \rho^{-}\left(L, f_{L}=0.96\right)$ & 2498 & 0.03 & $24.2_{-3.6}^{+3.0}$ & 0.96 & 2.6 & 45 \\
\hline$B^{0} \rightarrow f_{0} K^{*} 0_{K^{+} \pi^{-}}$ & 3359 & 0.54 & $\begin{array}{r}-3.6 \\
2^{*}\end{array}$ & 0.444 & 1.9 & 32 \\
\hline$B^{+} \rightarrow \omega \pi^{+}$ & 1248 & 0.07 & $6.7_{-06}^{+0.6}$ & 0.891 & 1.6 & 28 \\
\hline$B^{0} \rightarrow a_{1}^{0} K^{* 0}\left(L, f_{L}=0.7\right)$ & 5329 & 0.09 & $10^{*}$ & 0.467 & 1.5 & 26 \\
\hline$B^{+} \rightarrow K^{+} \pi^{0}$ & 1587 & 0.03 & $12.1_{-0.8}^{+0.8}$ & 1.000 & 1.5 & 26 \\
\hline$B^{0} \rightarrow \pi^{+} \pi^{-} K^{0}$ ( Dalitz) & 6816 & 0.02 & $44.8_{-2.5}^{+2.6}$ & 0.343 & 1 & 16 \\
\hline$B^{0} \rightarrow \rho^{-} K_{K^{+} \pi^{0}}^{*+}\left(L, f_{L}=0.4\right)$ & 2499 & 0.36 & $2^{*}$ & 0.333 & 0.9 & 16 \\
\hline$B^{0} \rightarrow \rho^{0} K_{K^{+} \pi^{-}}^{* 0}\left(T, f_{L}=0.5\right)$ & 2360 & 0.27 & $0.65_{-0.65}^{+0.65}$ & 0.667 & 0.4 & 7 \\
\hline$B^{+} \rightarrow \rho^{+} K_{K^{+} \pi^{-}}^{* 0}\left(T, f_{L}=0.5\right)$ & 2243 & 0.02 & $4.65_{-0.85}^{+0.85}$ & 0.666 & 0.3 & 4 \\
\hline$B^{+} \rightarrow \rho^{-} \pi^{+} \pi^{+}$ & 4151 & 0.01 & $5^{*}$ & 1.000 & 0.2 & 4 \\
\hline$B^{0} \rightarrow \rho^{-} K_{K^{+} \pi^{0}}^{*+}\left(T, f_{L}=0.4\right)$ & 2500 & 0.03 & $4^{*}$ & 0.333 & 0.1 & 2 \\
\hline Total & & & & & 794.8 & 13853 \\
\hline
\end{tabular}


Table 3.4: Potential $B \bar{B}$ background for the $B^{0} \rightarrow \eta_{\rho \gamma}^{\prime} K^{0}$ mode from exclusive charmless $B$ decays included in the $B \bar{B}$ background component. We show efficiency for the mode to pass selection cuts, the measured or estimated branching fraction, the appropriate product branching fraction given how the $\mathrm{MC}$ was produced, the estimated background normalized to 384 million $B \bar{B}$ events and the number of events we include in the file we use for making PDFs. An $*$ denotes an estimated branching fraction.

\begin{tabular}{|c|c|c|c|c|c|c|}
\hline Bkg. channel & Mode \# & $\begin{array}{c}\mathrm{MC} \epsilon \\
(\%)\end{array}$ & $\begin{array}{l}\text { Est. } \mathcal{B} \\
\left(10^{-6}\right)\end{array}$ & $\prod \mathcal{B}_{i}$ & $\begin{array}{l}\text { Norm. \# } \\
B \bar{B} \text { Bkg. }\end{array}$ & $\begin{array}{c}\# \text { in PDF } \\
\text { Bkg. file }\end{array}$ \\
\hline$B^{0} \rightarrow \pi^{+} \pi^{-} K^{0}$ ( Dalitz) & 6816 & 1.66 & $44.8_{-2.5}^{+2.6}$ & 0.343 & 98 & 3775 \\
\hline$B^{+} \rightarrow a_{1}^{+}\left(\rho^{0} \pi^{+}\right) K_{S}$ & 4959 & 2.32 & $15^{*}$ & 0.172 & 23 & 885 \\
\hline$B^{0} \rightarrow a_{1}^{0}\left(\rho^{-} \pi^{+}\right) K_{S}$ & 4955 & 2.14 & $15^{*}$ & 0.172 & 21.2 & 818 \\
\hline$B^{0} \rightarrow \phi_{3 \pi} K_{S}$ & 2714 & 6.39 & $8.3_{-1.0}^{+1.2}$ & 0.053 & 10.8 & 416 \\
\hline$B^{+} \rightarrow \rho^{+} K^{0}$ & 1933 & 0.89 & $5.6^{*}$ & 0.500 & 9.5 & 368 \\
\hline$B^{+} \rightarrow \rho K^{*}(1430)$ & 6102 & 0.05 & $40^{*}$ & 1.000 & 7.1 & 272 \\
\hline$B^{+} \rightarrow \rho^{0} K_{K}^{*+} \pi^{+}\left(L, f_{L}=1\right)$ & 2357 & 1.69 & $3.6_{-1.8}^{+1.9}$ & 0.229 & 5.4 & 206 \\
\hline$B^{+} \rightarrow a_{1}^{+}\left(\rho^{+} \pi^{0}\right) K_{S}$ & 4952 & 0.45 & $17^{*}$ & 0.172 & 5 & 193 \\
\hline$B^{0} \rightarrow \rho K^{*}(1430)$ & 6104 & 0.05 & $20^{*}$ & 1.000 & 4.1 & 158 \\
\hline$B^{0} \rightarrow K^{+} K^{-} K^{0}($ Dalitz $)$ & 6814 & 0.11 & $24.7_{-2.3}^{+2.3}$ & 0.343 & 3.7 & 141 \\
\hline$B^{0} \rightarrow \omega K_{S}$ & 1536 & 0.61 & $4.8_{-0.6}^{+0.6}$ & 0.306 & 3.5 & 133 \\
\hline$B^{+} \rightarrow \eta_{\rho \gamma}^{\prime} K_{K_{S} \pi^{+}}^{*+}$ & 2770 & 2.73 & $4.9_{-1.9}^{+2.1}$ & 0.067 & 3.4 & 132 \\
\hline$B^{0} \rightarrow \eta_{\eta \pi \pi}^{\prime} K_{S}$ & 1510 & 0.17 & $64.9_{-3.5}^{+3.5}$ & 0.060 & 2.6 & 99 \\
\hline$B^{+} \rightarrow \pi^{+} K_{S}$ & 991 & 0.07 & $24.1_{-1.3}^{+1.3}$ & 0.343 & 2.4 & 91 \\
\hline$B^{+} \rightarrow K_{K_{S} \pi^{0}}^{* 0} \pi^{+}$ & 1595 & 0.28 & $10.7_{-0.8}^{+0.8}$ & 0.167 & 2 & 75 \\
\hline$B^{0} \rightarrow \rho^{-} K_{K_{S} \pi^{+}}^{*+}\left(L, f_{L}=0.33\right)$ & 2501 & 0.35 & $2^{*}$ & 0.229 & 0.6 & 23 \\
\hline$B^{0} \rightarrow K^{0} K^{0}$ & 1774 & 0.82 & $0.95_{-0.23}^{+0.24}$ & 0.119 & 0.4 & 13 \\
\hline$B^{+} \rightarrow \phi_{3 \pi} K_{K_{S} \pi^{+}}^{*+}$ & 3994 & 0.17 & $9.7_{-1.5}^{+1.5}$ & 0.035 & 0.2 & 8 \\
\hline$B^{0} \rightarrow K^{+} K_{S} K_{S}$ & 3915 & 0.02 & $11.5_{-1.3}^{+1.3}$ & 0.119 & 0.1 & 4 \\
\hline$B^{0} \rightarrow \rho^{-} K_{K_{S} \pi^{+}}^{*+}\left(T, f_{L}=0.33\right)$ & 2502 & 0.03 & $4^{*}$ & 0.229 & 0.1 & 4 \\
\hline Total & & & & & 203.1 & 7814 \\
\hline
\end{tabular}


The same procedure is applied for $B^{+} \rightarrow \omega h^{+}$and the contributing backgrounds are shown in Table 3.5. We estimate a background of 453 events from charmless channels and include a single $B \bar{B}$ component in the fit.

For $\omega K_{S}^{0}$ the result of this procedure is shown in Table 3.6. A total of 46 charmless background events are estimated to enter into the fit. We construct a cocktail of these charmless MC events in the appropriate proportions and include them as a single component in the fit. The fit struggles to converge for $\omega K_{S}^{0}$ when $B \bar{B}$ is included as a floating component. We elect to fix the $B \bar{B}$ yield to the expected value of 46 and account for the uncertainty in this procedure with a systematic error.

\subsection{Fit Validation}

We generate "toy" MC samples matching the data in size with various assumptions about the signal content to assess the reliability of the fit. Fits are performed with all the floating parameters (yields, asymmetries and background PDF parameters) to test the stability of the fit.

\subsubsection{Validation of Yield Fits}

The toy validation studies for the yield fits are done without using $\Delta t$ to validate the fits that extract the signal yields and $\mathcal{A}_{c h}$.

\subsubsection{Pure Toy Studies}

In pure toy experiments, the PDFs for signal, charmless (where appropriate), and $q \bar{q}$ backgrounds are used to generate simulated events, which are used to form samples with the quantity of each component that we find in our final fit. We generate 1000 independent samples for each decay channel and perform the ML fit on each. The results are evaluated by considering the pull distribution for each variable that is floating in the fit. The pull distribution means generally are consistent with zero and the widths 
Table 3.5: Potential $B \bar{B}$ background for the $B^{+} \rightarrow \omega h^{+}$mode from exclusive charmless $B$ decays. We show the efficiency for the mode to pass selection cuts, the measured or estimated branching fraction, the appropriate product branching fraction, the estimated background normalized to 384 million $B \bar{B}$ events and the number of events we include in the file we use for making PDFs. An $*$ denotes an estimated branching fraction.

\begin{tabular}{|c|c|c|c|c|c|c|}
\hline Bkg. channel & Mode \# & $\begin{array}{c}\mathrm{MC} \epsilon \\
(\%)\end{array}$ & $\begin{array}{l}\text { Est. } \mathcal{B} \\
\left(10^{-6}\right)\end{array}$ & $\prod \mathcal{B}_{i}$ & $\begin{array}{l}\text { Norm. \# } \\
B \bar{B} \text { Bkg. }\end{array}$ & $\begin{array}{r}\text { \# in PDF } \\
\text { Bkg. file }\end{array}$ \\
\hline$B^{+} \rightarrow a_{1}^{0} \pi^{+}$ & 4156 & 0.7 & $20^{*}$ & 1.000 & 54.1 & 365 \\
\hline$B^{0} \rightarrow \pi^{-} a_{1}^{+}$ & 4157 & 0.35 & $39.7_{-3.7}^{+3.7}$ & 1.000 & 53.9 & 364 \\
\hline$B^{+} \rightarrow \omega \rho^{+}\left(L, f_{L}=0.88\right)$ & 2768 & 1.27 & $9.3_{-2.0}^{+2.3}$ & 0.891 & 40.5 & 273 \\
\hline$B^{0} \rightarrow a_{1}^{+}\left(\rho^{0} \pi^{+}\right) \pi^{-}$ & 1012 & 0.47 & $39.7_{-3.7}^{+3.7}$ & 0.500 & 35.8 & 241 \\
\hline$B^{+} \rightarrow \omega l^{-} \nu$ & 4761 & 0.07 & $130_{-60.0}^{+60.0}$ & 1.000 & 34.3 & 232 \\
\hline$B^{+} \rightarrow \eta_{\rho \gamma}^{\prime} K^{+}$ & 6748 & 0.36 & $69.7_{-2.7}^{+2.8}$ & 0.295 & 28.8 & 194 \\
\hline$B^{+} \rightarrow b_{1}^{0}\left(\omega \pi^{0}\right) \pi^{+}$ & 5273 & 0.76 & $10^{*}$ & 0.891 & 25.9 & 174 \\
\hline$B^{0} \rightarrow \pi^{+} \pi^{-} K^{0}$ ( Dalitz) & 6816 & 0.36 & $44.8_{-2.5}^{+2.6}$ & 0.343 & 21.1 & 142 \\
\hline$B^{+} \rightarrow a_{1}^{0} K^{+}$ & 4874 & 0.59 & $9^{*}$ & 1.000 & 20.3 & 137 \\
\hline$B^{0} \rightarrow b_{1}^{+}\left(\omega \pi^{+}\right) \pi^{-}$ & 5275 & 0.59 & $10^{*}$ & 0.891 & 20.2 & 136 \\
\hline$B^{0} \rightarrow a_{1}^{-}\left(\rho^{0} \pi^{-}\right) K^{+}$ & 4871 & 0.44 & $17^{*}$ & 0.500 & 14.3 & 96 \\
\hline$B^{+} \rightarrow b_{1}^{0}\left(\omega \pi^{0}\right) K^{+}$ & 5272 & 0.32 & $12^{*}$ & 0.891 & 13 & 87 \\
\hline$B^{+} \rightarrow \rho^{+} \rho^{0}\left(L, f_{L}=0.96\right)$ & 2390 & 0.16 & $18.3_{-3.4}^{+3.4}$ & 0.96 & 10.8 & 72 \\
\hline$B^{+} \rightarrow \pi^{+} K_{S}$ & 991 & 0.3 & $24.1_{-1.3}^{+1.3}$ & 0.343 & 9.7 & 65 \\
\hline$B^{0} \rightarrow \rho^{+} \rho^{-}\left(L, f_{L}=0.96\right)$ & 2498 & 0.09 & $24.2_{-3.6}^{+3.5}$ & 0.96 & 8 & 53 \\
\hline$B^{0} \rightarrow a_{1}^{-}\left(\rho^{-} \pi^{0}\right) K^{+}$ & 4960 & 0.24 & $15^{*}$ & 0.500 & 6.9 & 46 \\
\hline$B^{0} \rightarrow b_{1}^{+}\left(\omega \pi^{+}\right) K^{-}$ & 5274 & 0.31 & $6^{*}$ & 0.891 & 6.3 & 42 \\
\hline$B^{+} \rightarrow \eta_{\eta \pi \pi}^{\prime} K^{+}$ & 1506 & 0.13 & $69.7_{-2.7}^{+2.8}$ & 0.174 & 6.2 & 41 \\
\hline$B^{+} \rightarrow \phi_{3 \pi} K^{+}$ & 2713 & 1.24 & $8.3_{-0.65}^{+0.65}$ & 0.155 & 6.1 & 41 \\
\hline$B^{0} \rightarrow \rho^{+} K^{-}$ & 1044 & 0.15 & $9.9_{-1.5}^{+1.6}$ & 1.000 & 5.9 & 39 \\
\hline$B^{+} \rightarrow \pi^{+} l^{-} \nu$ & 1059 & 0.01 & $133_{-22.0}^{+22.0}$ & 2.000 & 5.5 & 36 \\
\hline$B^{+} \rightarrow \rho^{0} \pi^{+}$ & 1220 & 0.13 & $8.7_{-1.1}^{+1.0}$ & 1.000 & 4.4 & 29 \\
\hline$B^{0} \rightarrow \rho^{0} K^{0}$ & 1950 & 0.39 & $5.6_{-1.1}^{+1.1}$ & 0.5 & 4.2 & 28 \\
\hline$B^{+} \rightarrow \omega K^{+} \pi^{0}$ (N.R.) & 5578 & 0.03 & $20^{*}$ & 1.000 & 2.5 & 17 \\
\hline$B^{+} \rightarrow \eta_{3 \pi} K^{+}$ & 1515 & 1.11 & $2.5_{-0.3}^{+0.3}$ & 0.226 & 2.4 & 16 \\
\hline$B^{+} \rightarrow \rho^{+} \pi^{+} \pi^{-}$ & 2489 & 0.06 & $10^{*}$ & 1.000 & 2.4 & 16 \\
\hline$B^{+} \rightarrow K_{0}^{*} \pi$ & 3382 & 0.06 & $10.7_{-0.8}^{+0.8}$ & 1.0 & 2.3 & 15 \\
\hline$B^{+} \rightarrow \eta_{\eta_{3 \pi^{0} \pi \pi}^{\prime}}^{\prime} K^{+}$ & 6557 & 0.05 & $69.7_{-2.7}^{+2.8}$ & 0.144 & 2.1 & 13 \\
\hline$B^{+} \rightarrow \rho^{-} \pi^{+} \pi^{+}$ & 4151 & 0.08 & $5^{*}$ & 1.000 & 1.6 & 10 \\
\hline$B^{+} \rightarrow \pi^{+} \pi^{+} \pi^{-}($N.R. $)$ & 1230 & 0.13 & $3_{-3.0}^{+3.0}$ & 1.000 & 1.5 & 9 \\
\hline$B^{0} \rightarrow K_{K^{+} \pi^{0}}^{*+} \pi^{-}$ & 1226 & 0.1 & $9.8_{-1.1}^{+1.1}$ & 0.333 & 1.3 & 8 \\
\hline$B^{0} \rightarrow \rho^{0} K_{K^{+} \pi^{-}}^{* 0}\left(L, f_{L}=0.5\right)$ & 2359 & 0.21 & $0.65_{-0.65}^{+0.65}$ & 0.667 & 0.3 & 2 \\
\hline$B^{0} \rightarrow \rho^{0} \rho^{+} \pi^{-}(N . R)$. & 3837 & 0.01 & $10^{*}$ & 1.000 & 0.2 & 1 \\
\hline$B^{+} \rightarrow \omega \rho^{+}\left(T, f_{L}=0.88\right)$ & 2766 & 0.05 & $1.3_{-0.3}^{+0.3}$ & 0.891 & 0.2 & 1 \\
\hline$B^{0} \rightarrow \pi^{+} \pi^{-} \pi^{0}$ & 1222 & 0.04 & $1^{*}$ & 1.000 & 0.2 & 1 \\
\hline Total & & & & & 453.4 & 3042 \\
\hline
\end{tabular}


Table 3.6: Potential $B \bar{B}$ background for the $B^{0} \rightarrow \omega K^{0}$ mode from exclusive charmless $B$ decays. We show the efficiency for the mode to pass selection cuts, the measured or estimated branching fraction, the appropriate product branching fraction, the estimated background normalized to 384 million $B \bar{B}$ events and the number of events we include in the file we use for making PDFs. An $*$ denotes an estimated branching fraction.

\begin{tabular}{|c|c|c|c|c|c|c|}
\hline Bkg. channel & Mode \# & $\begin{array}{c}\mathrm{MC} \epsilon \\
(\%)\end{array}$ & $\begin{array}{l}\text { Est. } \mathcal{B} \\
\left(10^{-6}\right)\end{array}$ & $\prod \mathcal{B}_{i}$ & $\begin{array}{l}\text { Norm. \# } \\
B \bar{B} \text { Bkg. }\end{array}$ & $\begin{array}{c}\# \text { in PDF } \\
\text { Bkg. file }\end{array}$ \\
\hline$B^{0} \rightarrow \eta_{\rho \gamma}^{\prime} K_{S}$ & 6749 & 0.42 & $64.9_{-3.5}^{+3.5}$ & 0.101 & 10.5 & 636 \\
\hline$B^{0} \rightarrow \eta_{\omega \gamma}^{\prime} K_{S}$ & 5319 & 4.25 & $64.9_{-3.5}^{+3.5}$ & 0.009 & 9.5 & 578 \\
\hline$B^{0} \rightarrow b_{1}^{0}\left(\omega \pi^{0}\right) K_{S}$ & 5276 & 0.85 & $8^{*}$ & 0.306 & 8 & 484 \\
\hline$B^{0} \rightarrow a_{1}^{0}\left(\rho^{-} \pi^{+}\right) K_{S}$ & 4955 & 0.77 & $15^{*}$ & 0.172 & 7.7 & 464 \\
\hline$B^{+} \rightarrow b_{1}^{+}\left(\omega \pi^{+}\right) K_{S}$ & 5270 & 0.8 & $5^{*}$ & 0.306 & 4.7 & 286 \\
\hline$B^{0} \rightarrow \pi^{+} \pi^{-} K^{0}$ (Dalitz) & 6816 & 0.05 & $44.8_{-2.5}^{+2.6}$ & 0.343 & 3.1 & 186 \\
\hline$B^{+} \rightarrow K_{K_{S} \pi^{+}}^{*+} K_{S}$ & 1944 & 0.88 & $0.95^{*}$ & 0.333 & 1.1 & 64 \\
\hline$B^{+} \rightarrow \omega K_{K_{S} \pi^{+}}^{*+}\left(f_{L}=1\right)$ & 2505 & 1.84 & $0.6_{-0.6}^{+1.8}$ & 0.204 & 0.9 & 52 \\
\hline$B^{0} \rightarrow K^{+} K_{S} K_{S}$ & 3915 & 0.04 & $11.5_{-1.3}^{+1.3}$ & 0.119 & 0.2 & 11 \\
\hline Total & & & & & 45.7 & 2761 \\
\hline
\end{tabular}


are consistent with one, as expected. The pull tables are given in App. E.

\subsubsection{Embedded Toy Studies}

In embedded toy experiments, $q \bar{q}$ backgrounds are generated from the PDFs as in pure toys, and samples of MC events are embedded for signal and charmless backgrounds. This allows for the determination of the bias on our signal due to the presence of the charmless events, and also to understand the effect of correlations between variables in the signal and charmless events. From these studies, we determine a fit bias on the signal yield that is used as an additive correction. The results of these embedded toy studies are given in Table 3.7 .

Table 3.7: Summary of results from embedded toy MC studies. We use 1000 toy experiments, embedding the number of signal and charmless $\mathrm{MC}$ events given below. All numbers are in events.

\begin{tabular}{lrrrrr}
\hline \hline Final state & $\begin{array}{c}\text { Total toy } \\
\text { events }\end{array}$ & $\begin{array}{c}\text { \# signal } \\
\text { input }\end{array}$ & $\begin{array}{c}\# B \bar{B} \\
\text { input }\end{array}$ & $\begin{array}{c}\text { Mean \# } \\
\text { signal fit }\end{array}$ & $\begin{array}{c}\text { Difference } \\
\text { (bias) }\end{array}$ \\
\hline$\eta_{\eta \pi \pi}^{\prime} K^{+}$ & 3170 & 1060 & - & $1060.0 \pm 0.5$ & $0.0 \pm 0.5$ \\
$\eta_{\rho \gamma}^{\prime} K^{+}$ & 79501 & 2375 & 795 & $2406.0 \pm 1.5$ & $31.0 \pm 1.5$ \\
$\eta_{\eta \pi \pi}^{\prime} K^{0}$ & 1100 & 329 & - & $331.8 \pm 0.3$ & $2.8 \pm 0.3$ \\
$\eta_{\rho \gamma}^{\prime} K^{0}$ & 19927 & 795 & 203 & $829.7 \pm 0.8$ & $34.7 \pm 0.8$ \\
$\omega \pi^{+}$ & 76735 & 470 & 200 & $513.7 \pm 1.5$ & $43.7 \pm 1.5$ \\
$\omega K^{+}$ & 76735 & 428 & 113 & $457.3 \pm 0.9$ & $29.3 \pm 0.9$ \\
$\omega K_{S}^{0}$ & 15914 & 137 & 46 & $147.3 \pm 0.4$ & $10.3 \pm 0.4$ \\
\hline \hline
\end{tabular}

\subsection{2 $\mathcal{A}_{c h}$ Bias Effect}

The presence of a positive bias in the fit yield may cause a dilution in the measured charge asymmetry. The signal $\mathrm{MC}$ is generated with equal numbers of $B^{+}$and $B^{-}$ events. To test this effect, we create subsamples of the MC with $\mathcal{A}_{c h}=0.300$ for both $\omega K^{+}$and $\omega \pi^{+}$. These samples are used in toy experiments with the results reported in Table 3.8. A pure dilution bias from the fit bias would be a factor of 1.09 for $\omega \pi^{+}$ 
and a factor of 1.07 for $\omega K^{+}$. This bias would cause the fit $\mathcal{A}_{c h}$ and the uncertainty to be smaller than expected. The toys show less than half of this expected bias is actually observed in our fit. Some of the fit bias, therefore, may not contribute a dilution effect. To account for this effect, we scale the central value and statistical error by the dilution factor found in the toy experiments of $0.300 / 0.293=1.02$ for $\omega K^{+}$and $0.300 / 0.288=1.04$ for $\omega \pi^{+}$.

Table 3.8: Summary of results from embedded toy MC studies in $\omega h^{+}$. We use 500 toy experiments, embedding signal MC events with the $\mathcal{A}_{c h}$ as indicated in the table. All numbers are in events.

\begin{tabular}{rrrr}
\hline \hline \multicolumn{2}{c}{ Embedded $\mathcal{A}_{c h}$} & \multicolumn{2}{c}{ Fit $\mathcal{A}_{c h}$} \\
$\omega K^{+}$ & $\omega \pi^{+}$ & $\omega K^{+}$ & \multicolumn{1}{c}{$\omega \pi^{+}$} \\
\hline-0.006 & -0.007 & $-0.000 \pm 0.003$ & $-0.007 \pm 0.003$ \\
0.300 & -0.007 & $0.293 \pm 0.003$ & $-0.006 \pm 0.003$ \\
-0.006 & 0.300 & $0.002 \pm 0.003$ & $0.288 \pm 0.003$ \\
0.300 & 0.300 & $0.296 \pm 0.003$ & $0.288 \pm 0.003$ \\
\hline \hline
\end{tabular}

The fit bias is small $\left(\lesssim 1 \%\right.$ ) in the $\eta^{\prime} K^{+}$channels, so we conclude the effect from a dilution is negligible.

\subsubsection{Validation of $C P$ Fits}

The toy validation studies for the $C P$ fits are done with $\Delta t$ and tagging category information as in the final fits to extract $C P$ parameters, $S$ and $C$. For toy studies the signal $\Delta t$ shapes are taken from signal MC as we find they are slightly different from those found for data.

\subsubsection{Pure Toy Studies}

For the time-dependent fit channels, pure toys are performed with the number of signal, charmless and $q \bar{q}$ events generated according to the yields found in the final 
fits to data. For $\eta_{\rho \gamma}^{\prime} K^{0} 500$ pure toy experiments are performed with $C=-0.3$ and $S=0.5$, and for $\eta_{\eta \pi \pi}^{\prime} K^{0}$ signal events are generated with $C=-0.25$ and $S=0.6$. As expected, all pull distribution results are consistent with a mean of zero and a width of one, as shown in App. E.

For $\omega K_{S}^{0}, 3000$ pure toy experiments are generated with $C=-0.43$ and $S=0.62$. Most fit variables have pull distributions with means consistent with zero and widths consistent with one, as shown in App. E. There is some bias, however, in $S$ and $C$ in both central value and error. The central value and pull distributions for $S$ and $C$ from these toy experiments are shown in Fig. 3.4. This issue is investigated further in Sec. 4.2 .3 .

\subsubsection{Embedded Toy Studies}

Embedded toy studies for the time-dependent fits are used to understand any potential bias on $S$ or $C$. Where measured, the $C P$ content of charmless background modes is included in the MC.

We summarize in Table 3.9 the mean values of the signal yield and of the asymmetry parameters with corresponding errors for the $B^{0} \rightarrow \eta^{\prime} K^{0}$ modes.

Table 3.9: Mean values of $C P$-violating parameters and their errors for 1000 embedded toy MC experiments for $\eta^{\prime} K_{S}^{0}$. We embed 770 signal and $360 B \bar{B}$ events in $q \bar{q}$ samples generated from PDF background for $\eta_{\rho \gamma}^{\prime} K^{0}$, and 320 signal events in $q \bar{q}$ samples generated from PDF background for $\eta_{\eta \pi \pi}^{\prime} K^{0}$. The embedded $\mathrm{MC}$ is generated with the values $S=0.7$ and $C=0.0$.

\begin{tabular}{lcccccc}
\hline \hline & & \multicolumn{2}{c}{$\eta_{\rho \gamma}^{\prime} K^{0}$} & \multicolumn{3}{c}{$\eta_{\eta \pi \pi}^{\prime} K^{0}$} \\
& Signal yield & \multicolumn{1}{c}{$C$} & Signal yield & \multicolumn{1}{c}{$S$} & $C$ \\
\hline Quantity & $790.0 \pm 0.7$ & $0.670 \pm 0.004$ & $-0.001 \pm 0.003$ & $320.5 \pm 0.2$ & $0.692 \pm 0.007$ & $0.002 \pm 0.005$ \\
Error & $35.5 \pm 0.1$ & $0.125 \pm 0.001$ & $0.097 \pm 0.001$ & $19.3 \pm 0.01$ & $0.193 \pm 0.001$ & $0.141 \pm 0.001$ \\
\hline \hline
\end{tabular}

We conclude from these sets of toys that there is evidence of a bias of -0.030 in $S$ for the $\eta_{\rho \gamma}^{\prime} K^{0}$ mode. This is connected with the amount of $B \bar{B}$ background used in 

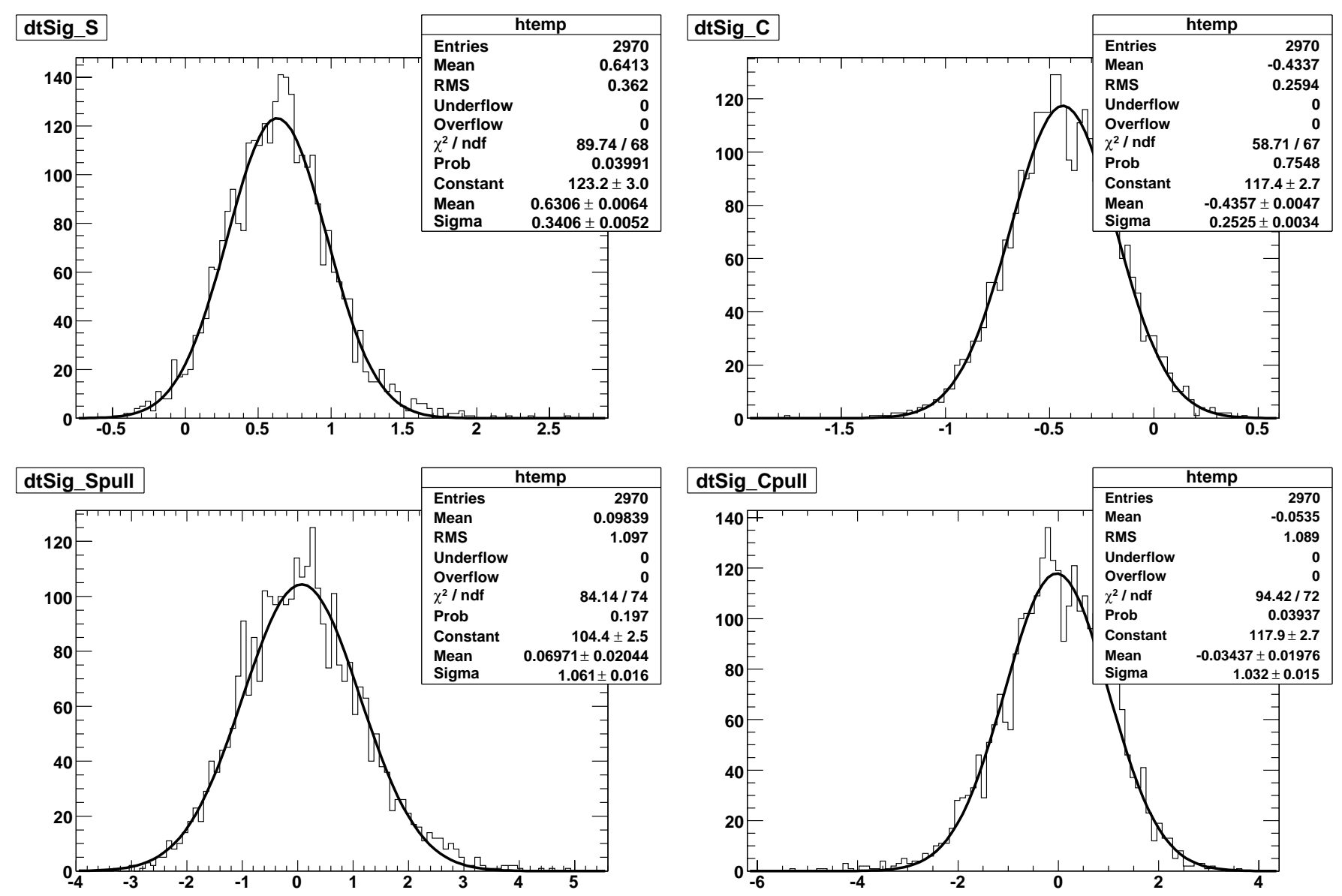

Figure 3.4: Central value (top) and pull (bottom) distributions for pure toy experiments for $B^{0} \rightarrow \omega K^{0}$ for $S$ (left) and $C$ (right) generated with $S=0.62$ and $C=-0.43$. 
the toy fits and is discussed further in Sec. 4.2.2. We apply a correction for the dilution due to $B \bar{B}$ of 0.01 on $S$ for $\eta_{\rho \gamma}^{\prime} K^{0}$.

We perform 3000 embedded toy MC experiments for $\omega K_{S}^{0}$. We summarize in Table 3.10 the mean values of signal yield and of the asymmetry parameters with corresponding errors. We conclude from these sets of toys that there is evidence of a bias of +0.015 in $S$ and apply a correction to account for this.

Table 3.10: Mean values of $C P$-violating parameters and their errors for $\omega K_{S}^{0}$ for 3000 embedded toy MC experiments. We embed 143 signal and $35 B \bar{B}$ events in samples of $13647 q \bar{q}$ background events. The embedded MC is generated with the values $S=0.7$ and $C=0.0$.

\begin{tabular}{lcccr}
\hline \hline$\omega K_{S}^{0}$ & Signal yield & Charmless yield & $S$ & \multicolumn{1}{c}{$C$} \\
\hline Quantity & $149.8 \pm 0.2$ & $14.4 \pm 0.5$ & $0.715 \pm 0.007$ & $-0.003 \pm 0.005$ \\
Error & $17.0 \pm 0.1$ & $23.9 \pm 0.1$ & $0.324 \pm 0.001$ & $0.246 \pm 0.001$ \\
\hline \hline
\end{tabular}

\subsubsection{BReco MC Parameters}

The nominal toy studies are performed using the signal MC parameters for the signal $\Delta t$ shape and tagging category fractions. We also perform toy studies using BReco signal MC for these parameters for $\eta_{\eta \pi \pi}^{\prime} K^{0}$. The results in Table 3.11 indicate that there is no significant difference between the two methods, as expected since most differences are accounted for by $\sigma_{\Delta t}$ when $\Delta t / \sigma_{\Delta t}$ is used. 
Table 3.11: Comparison of the nominal toy fits for $\eta_{\eta \pi \pi}^{\prime} K^{0}$, where signal MC is used for signal $\Delta t$ parameters, and a set of 500 toy experiments with BReco MC for the signal $\Delta t$ parameters. There are 275 signal MC events embedded in both cases.

\begin{tabular}{lccr}
\hline \hline MC used & Signal yield & $S$ & \multicolumn{1}{c}{$C$} \\
\hline Signal & $273.2 \pm 0.1$ & $0.684 \pm 0.004$ & $0.007 \pm 0.003$ \\
BReco & $273.3 \pm 0.3$ & $0.688 \pm 0.009$ & $-0.005 \pm 0.006$ \\
\hline \hline
\end{tabular}




\title{
Chapter 4
}

\author{
Results
}

\subsection{Branching Fractions and Charge Asymmetry Results}

This section presents the results of the branching fraction and charge asymmetry fits.

\subsubsection{Branching Fraction Fit Results}

The results for the $B \rightarrow \eta^{\prime} K$ modes are shown in Table 4.1. Table 4.2 shows the results for the $\omega$ modes. The statistical error on the signal yield is equal to the change in value that corresponds to an increase of $-2 \ln \mathcal{L}$ by one unit from its minimum. The significance is equal to the square root of the difference between the value of $-2 \ln \mathcal{L}$ for zero signal and the value at its minimum. The inclusion of systematic errors in the significance is described in App. F.

The $\eta_{\eta \pi \pi}^{\prime}$ and $\eta_{\rho \gamma}^{\prime}$ submodes are combined using the method of combining $-2 \ln \mathcal{L}$, which is described in App. F. The results of the combining procedure for the branching fractions are shown in Fig. 4.1. The combined results for $\mathcal{A}_{c h}$ are shown in Fig. 4.2. 
Table 4.1: Branching fraction and charge asymmetry results for the $B \rightarrow \eta^{\prime} K$ modes.

\begin{tabular}{|c|c|c|c|c|}
\hline Analysis & $\eta_{\eta \pi \pi}^{\prime} K^{+}$ & $\eta_{\rho \gamma}^{\prime} K^{+}$ & $\eta_{\eta \pi \pi}^{\prime} K^{0}$ & $\eta_{\rho \gamma}^{\prime} K^{0}$ \\
\hline Events into fit & 3170 & 79501 & 1100 & 19927 \\
\hline Signal yield & $1059.9 \pm 35.4$ & $2405.0 \pm 68.8$ & $329.3 \pm 20.0$ & $831.2 \pm 38.5$ \\
\hline$B \bar{B}$ yield & - & $1109 \pm 161$ & - & $136 \pm 81$ \\
\hline \# Data combs/event & 1.050 & 1.072 & 1.084 & 1.075 \\
\hline \# MC combs/event & 1.059 & 1.072 & 1.104 & 1.074 \\
\hline ML-fit bias (events) & 0.0 & 31.0 & 2.8 & 34.7 \\
\hline $\mathrm{MC} \epsilon(\%)$ & 23.6 & 29.2 & 23.8 & 28.2 \\
\hline Neutrals corr. (\%) & 98.3 & - & 98.3 & - \\
\hline$K_{S}^{0}$ corr. $(\%)$ & - & - & 99.3 & 99.3 \\
\hline Corr. $\epsilon(\%)$ & 23.2 & 29.2 & 23.2 & 28.0 \\
\hline$\prod \mathcal{B}_{i}(\%)$ & 17.5 & 29.4 & 6.06 & 10.2 \\
\hline Corr. $\epsilon \times \prod \mathcal{B}_{i}(\%)$ & 4.06 & 8.58 & 1.41 & 2.86 \\
\hline $\mathcal{B}\left(\times 10^{-6}\right)$ & $68.2 \pm 2.3 \pm 3.3$ & $72.2 \pm 2.1 \pm 3.2$ & $60.7 \pm 3.7 \pm 3.0$ & $72.8 \pm 3.5 \pm 3.7$ \\
\hline Combined $\mathcal{B}\left(\times 10^{-6}\right)$ & \multicolumn{2}{|c|}{$70.0 \pm 1.5 \pm 2.8$} & \multicolumn{2}{|c|}{$66.6 \pm 2.6 \pm 2.8$} \\
\hline Stat. sign. $(\sigma)$ (stat. only) & \multicolumn{2}{|c|}{79} & \multicolumn{2}{|c|}{43} \\
\hline Stat. sign. $(\sigma)$ & \multicolumn{2}{|c|}{70} & \multicolumn{2}{|c|}{38} \\
\hline Background $\mathcal{A}_{c h}$ & $-0.033 \pm 0.025$ & $-0.017 \pm 0.004$ & - & - \\
\hline Corr. Signal $\mathcal{A}_{c h}$ & $-0.005 \pm 0.033 \pm 0.005$ & $0.022 \pm 0.028 \pm 0.008$ & - & - \\
\hline Combined Signal $\mathcal{A}_{c h}$ & \multicolumn{2}{|c|}{$0.010 \pm 0.022 \pm 0.006$} & \multicolumn{2}{|c|}{-} \\
\hline
\end{tabular}

Table 4.2: Branching fraction and charge asymmetry results for the $\omega$ modes.

\begin{tabular}{|c|c|c|c|}
\hline Analysis & $\omega \pi^{+}$ & $\omega K^{+}$ & $\omega K_{S}^{0}$ \\
\hline Events into fit & \multicolumn{2}{|c|}{76735} & 15914 \\
\hline Signal yield & $515.6 \pm 38.1$ & $456.6 \pm 32.2$ & $145.5 \pm 18.1$ \\
\hline$B \bar{B}$ yield & $206.8 \pm 142.5$ & $113.2 \pm 88.6$ & 46(fixed) \\
\hline \# Data combs/event & 1.117 & 1.117 & 1.132 \\
\hline \# MC combs/event & 1.113 & 1.099 & 1.124 \\
\hline ML-fit bias (events) & 43.7 & 29.3 & 10.3 \\
\hline $\mathrm{MC} \epsilon(\%)$ & 21.2 & 20.7 & 22.1 \\
\hline Neutrals corr. (\%) & 96.8 & 96.8 & 96.8 \\
\hline$K_{S}^{0}$ corr. $(\%)$ & - & - & 95.7 \\
\hline Corr. $\epsilon(\%)$ & 20.5 & 20.0 & 20.4 \\
\hline$\prod \mathcal{B}_{i}(\%)$ & 89.1 & 89.1 & 30.8 \\
\hline Corr. $\epsilon \times \prod \mathcal{B}_{i}(\%)$ & 18.3 & 17.8 & 6.3 \\
\hline $\mathcal{B}\left(\times 10^{-6}\right)$ & $6.7 \pm 0.5 \pm 0.4$ & $6.3 \pm 0.5 \pm 0.3$ & $5.6 \pm 0.8 \pm 0.3$ \\
\hline Stat. sign. $(\sigma)$ (stat. only) & 17.0 & 19.0 & 11.1 \\
\hline Stat. sign. $(\sigma)$ & 12.4 & 15.3 & 10.0 \\
\hline Background $\mathcal{A}_{c h}$ & $-0.007 \pm 0.004$ & $-0.006 \pm 0.004$ & - \\
\hline Corr. Signal $\mathcal{A}_{c h}$ & $-0.020 \pm 0.075 \pm 0.006$ & $-0.007 \pm 0.071 \pm 0.005$ & - \\
\hline
\end{tabular}



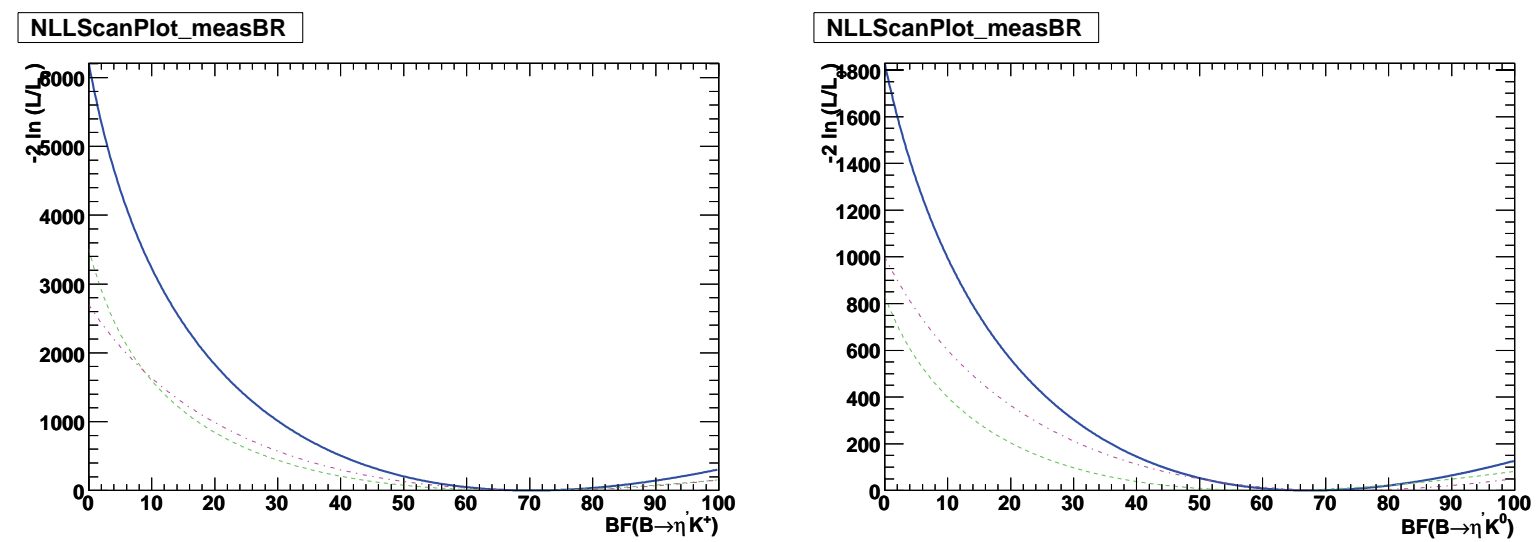

Figure 4.1: The distribution of $-2 \ln \mathcal{L}$ for $\eta^{\prime} K^{+}$(left) and $\eta^{\prime} K_{S}^{0}$ (right) branching fractions. The pink dot-dashed curve shows the $-2 \ln \mathcal{L}$ distribution for the $\eta^{\prime} \rightarrow \rho^{0} \gamma$ submode, the green dashed curve is for the $\eta^{\prime} \rightarrow \eta \pi^{+} \pi^{-}$submode, and the blue solid curve is the combined result.

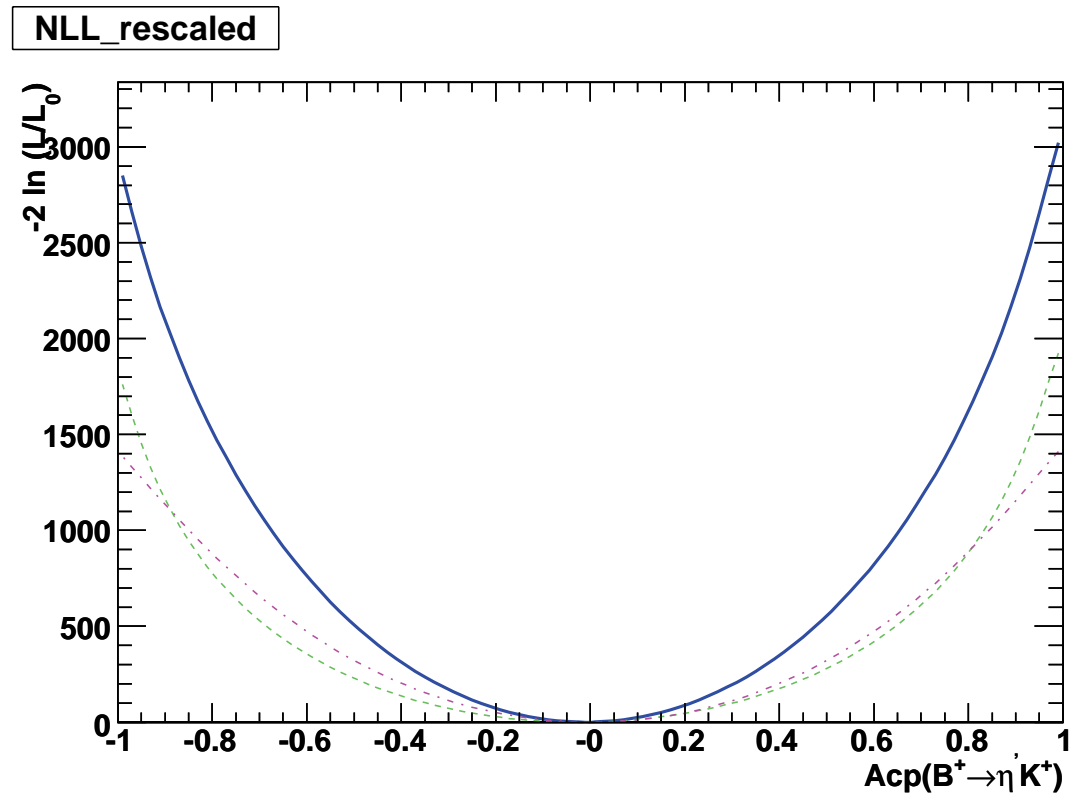

Figure 4.2: The distribution of $-2 \ln \mathcal{L}$ for $\mathcal{A}_{c h}\left(\eta^{\prime} K^{+}\right)$The pink dot-dashed curve shows the $-2 \ln \mathcal{L}$ distribution for the $\eta^{\prime} \rightarrow \rho^{0} \gamma$ submode, the green dashed curve is for the $\eta^{\prime} \rightarrow \eta \pi^{+} \pi^{-}$submode, and the blue solid curve is the combined result. 


\subsubsection{Projection Plots}

In Fig. 4.3 we show projections of $m_{\mathrm{ES}}$ and $\Delta E$ for $\eta_{\eta \pi \pi}^{\prime} K^{+}, \eta_{\rho \gamma}^{\prime} K^{+}, \eta_{\eta \pi \pi}^{\prime} K^{0}$, and $\eta_{\rho \gamma}^{\prime} K^{0}$. In each case a subset of the data is used for which the signal likelihood (computed without the variable plotted) exceeds a mode-dependent threshold that optimizes the sensitivity. Figures 4.4-4.6 show projections of $m_{\mathrm{ES}}, \Delta E, \mathrm{~m}_{\omega}, \mathcal{H}_{\omega}$, and $\mathcal{F}$ for $\omega \pi^{+}$, $\omega K^{+}$, and $\omega K_{S}^{0}$. In all cases, the projections show clear signs of signal events peaking at zero in $\Delta E$ and $m_{B}$ in $m_{\mathrm{ES}}$.
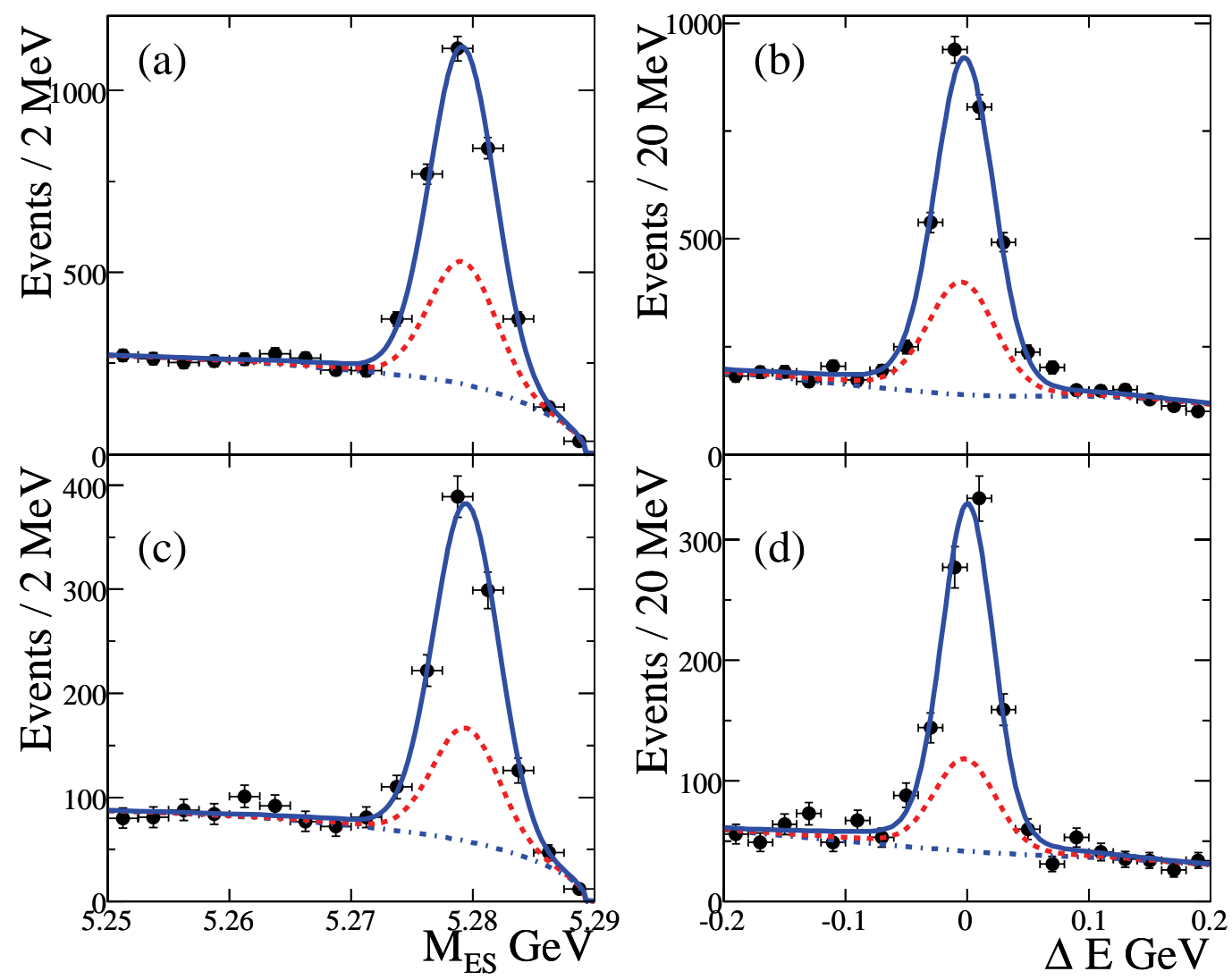

Figure 4.3: The $B$ candidate $m_{\mathrm{ES}}$ projections for (a) $\eta^{\prime} K^{+}$and (c) $\eta^{\prime} K_{S}^{0}$ and $\Delta E$ projections for (b) $\eta^{\prime} K^{+}$and (d) $\eta^{\prime} K_{S}^{0}$. Points with errors represent the data for both submodes combined, blue solid curves the full fit functions, red dashed curves the background functions plus the $\eta_{\eta \pi \pi}^{\prime}$ signal, and the blue dot-dashed curves the background functions. 

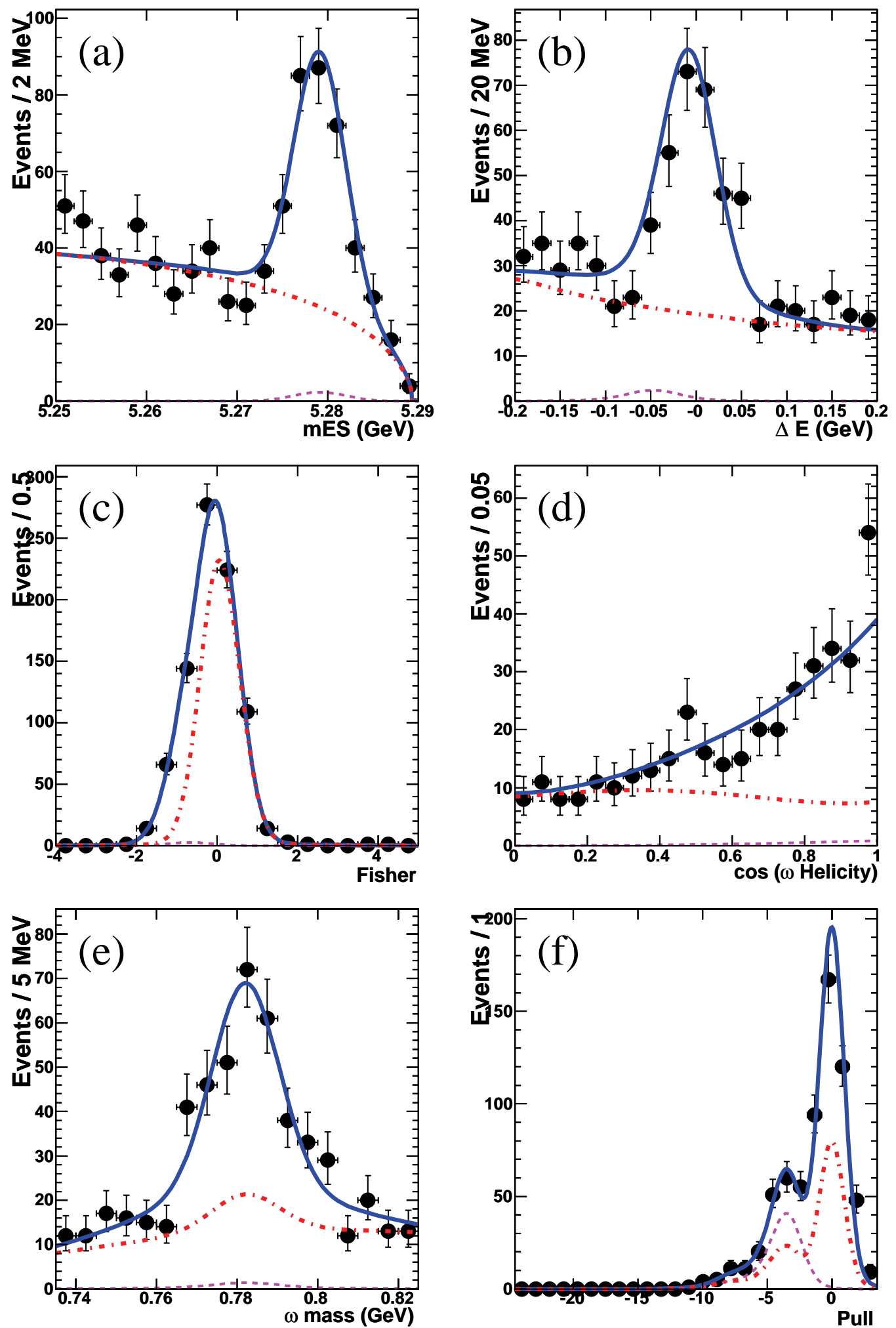

Figure 4.4: The $B$ candidate projections for $\omega \pi^{+}$for (a) $m_{\mathrm{ES}}$, (b) $\Delta E$, (c) $\mathrm{m}_{\omega}$, (d) $\mathcal{H}_{\omega}$, (e) $\mathcal{F}$ and (f) $S_{\pi}$. Points with errors represent the data, solid blue curves the full fit functions, red dot-dashed curves the sum of the background functions, and the pink dashed curves the signal from the crossfeed mode. 

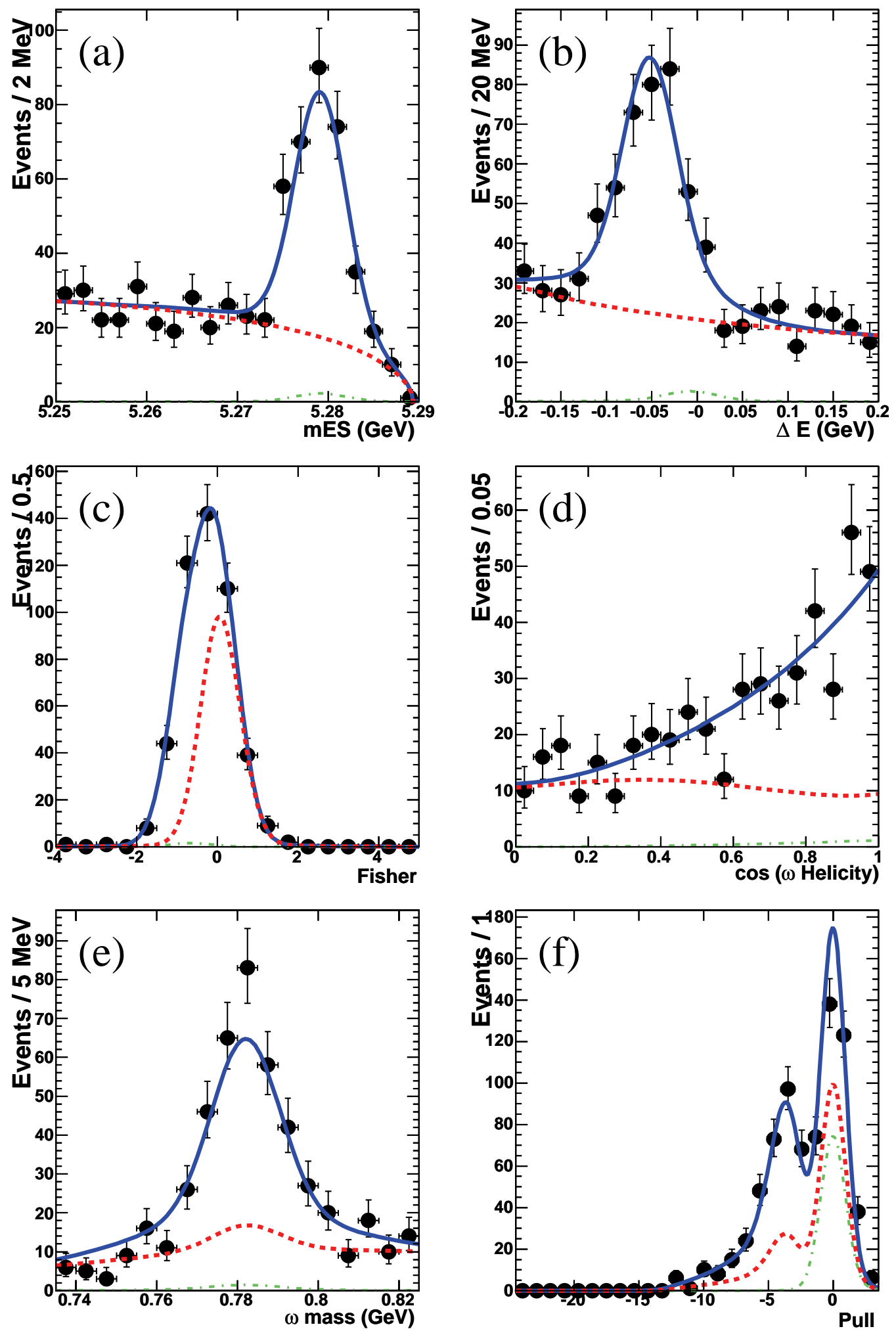

Figure 4.5: The $B$ candidate projections for $\omega K^{+}$for (a) $m_{\mathrm{ES}}$, (b) $\Delta E$, (c) $\mathrm{m}_{\omega}$, (d) $\mathcal{H}_{\omega}$, (e) $\mathcal{F}$ and (f) $S_{\pi}$. Points with errors represent the data, solid blue curves the full fit functions, red dot-dashed curves the sum of the background functions, and the green dot-dashed curves the signal from the crossfeed mode. 

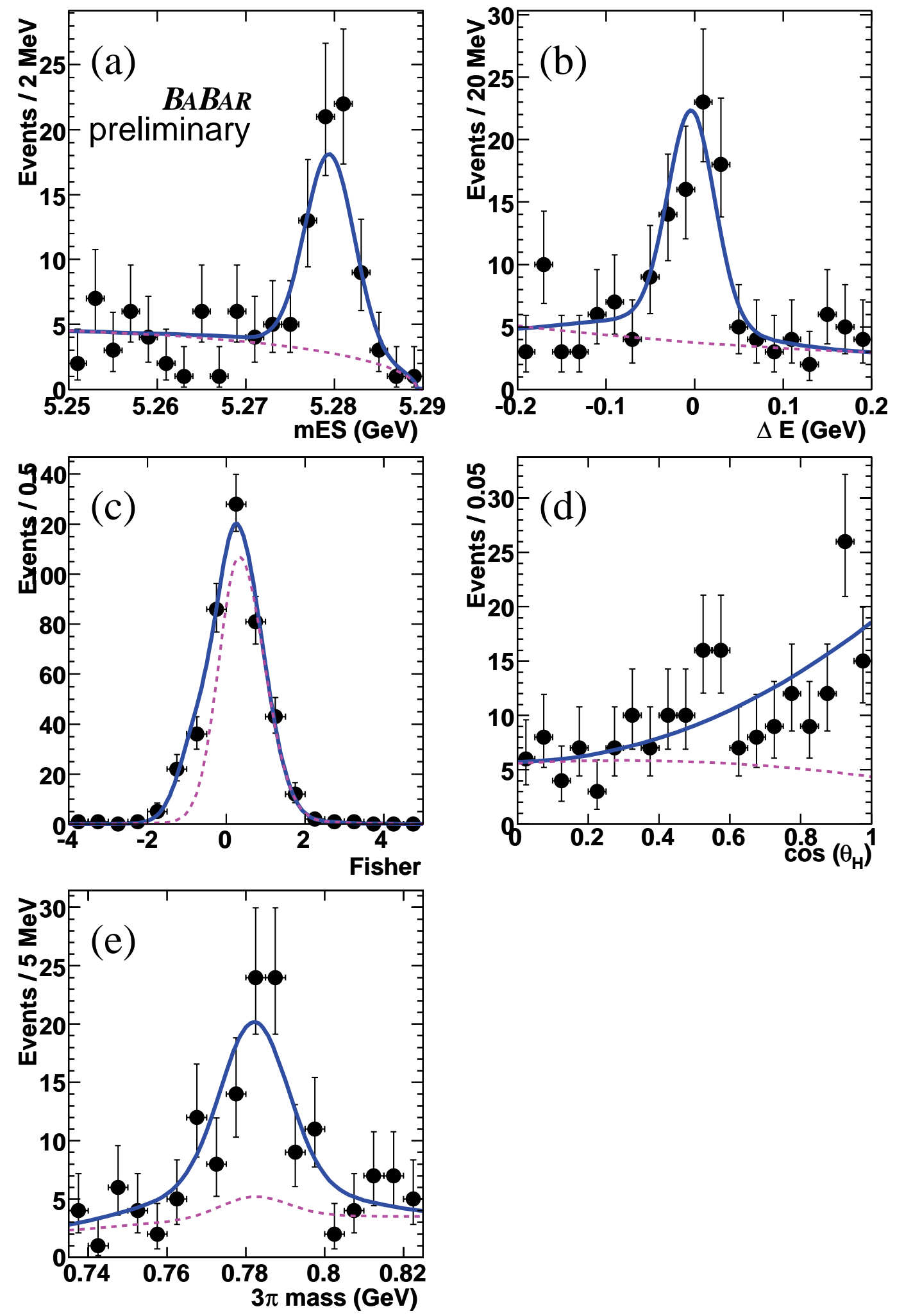

Figure 4.6: The $B$ candidate projections for $\omega K_{S}^{0}$ for (a) $m_{\mathrm{ES}}$, (b) $\Delta E$, (c) $\mathrm{m}_{\omega}$, (d) $\mathcal{H}_{\omega}$, and (e) $\mathcal{F}$. Points with errors represent the data, solid blue curves the full fit functions and pink dashed curves the sum of the background functions. 


\subsection{3 splots}

In Figures 4.7-4.10 we show sPlots of the signal and background components of $m_{\mathrm{ES}}, \Delta E$, and $\mathcal{F}$ for $\eta_{\eta \pi \pi}^{\prime} K^{+}, \eta_{\rho \gamma}^{\prime} K^{+}, \eta_{\eta \pi \pi}^{\prime} K^{0}$, and $\eta_{\rho \gamma}^{\prime} K^{0}$. The $s$ Plot technique [52] uses the PDFs to weight appropriately each event by its component likelihood. For signal sPlots a higher weight is given to the most signal-like events and the weights for background are zero on average. In this way, no cuts are needed to visually enhance the events for the component plotted. Figures 4.11-4.13 show the sPlots of $m_{\mathrm{ES}}, \Delta E, \mathrm{~m}_{\omega}, \mathcal{H}_{\omega}$, and $\mathcal{F}$ for signal, $q \bar{q}$ background, and charmless background for $\omega \pi^{+}, \omega K^{+}$, and $\omega K_{S}^{0}$. In each plot, the PDF curve is overlaid on the sPlot data and the component plotted is not included in the sPlot fit. Good agreement between the data and curves shows that the PDFs used in the fit are actually good representations of the shapes found in the data. 

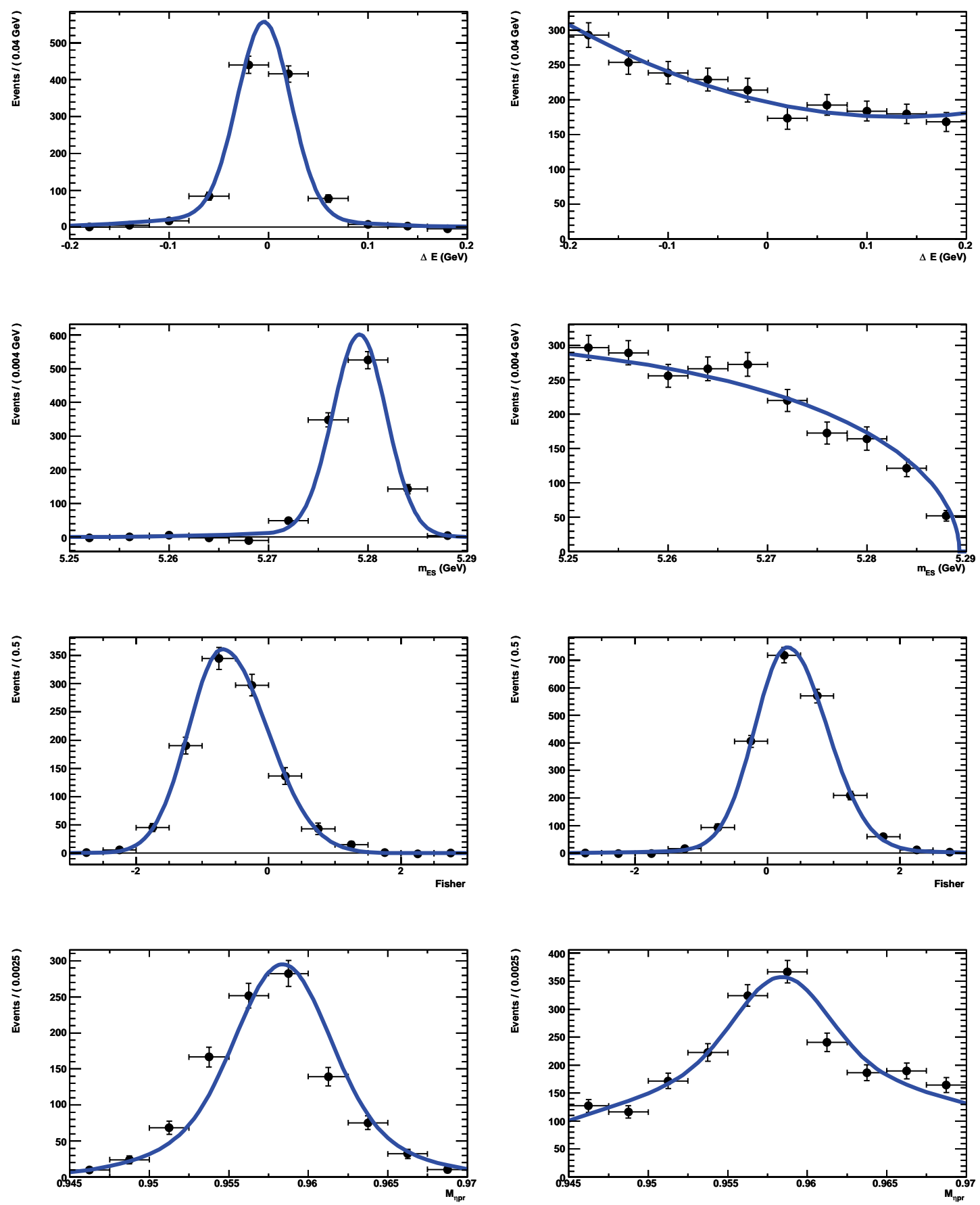

Figure 4.7: From top to bottom, the $B$ candidate $m_{\mathrm{ES}}, \Delta E, \mathcal{F}$, and $m_{\eta^{\prime}}$ sPlots for $\eta_{\eta \pi \pi}^{\prime} K^{+}$. The bottom row of plots is for information only and is not a variable used in the fit. The left column is signal and the right column is $q \bar{q}$ background. Signal yields are $1060 \pm 39,1045 \pm 37$, and $1081 \pm 37$ when $m_{\mathrm{ES}}, \Delta E$, or $\mathcal{F}$ is removed from the fit. The nominal yield is $1060 \pm 35$. 

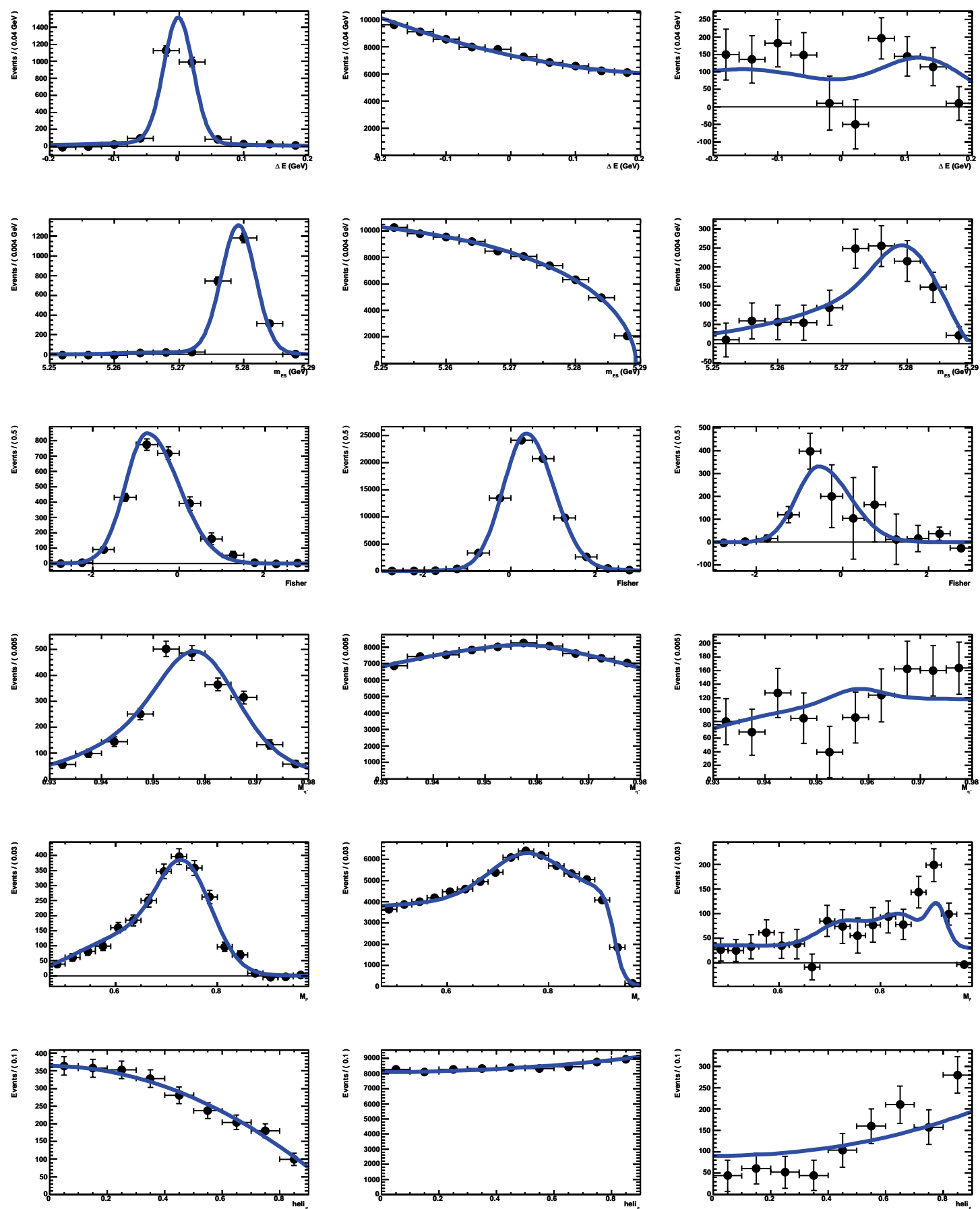

Figure 4.8: From top to bottom, the $B$ candidate $\Delta E, m_{\mathrm{ES}}, \mathcal{F}, m_{\eta^{\prime}}, m_{\rho}$, and $\mathcal{H}_{\rho}$ $s$ Plots for $\eta_{\rho \gamma}^{\prime} K^{+}$. The bottom three rows of plots are for information only and are not variables used in the fit. The left column is signal, middle column is $q \bar{q}$ background and the right column is charmless background. Signal yields are $2270 \pm 85,2358 \pm 122$, and $2628 \pm 90$ when $m_{\mathrm{ES}}, \Delta E$, or $\mathcal{F}$ is removed from the fit. The nominal yield is $2405 \pm 69$. 

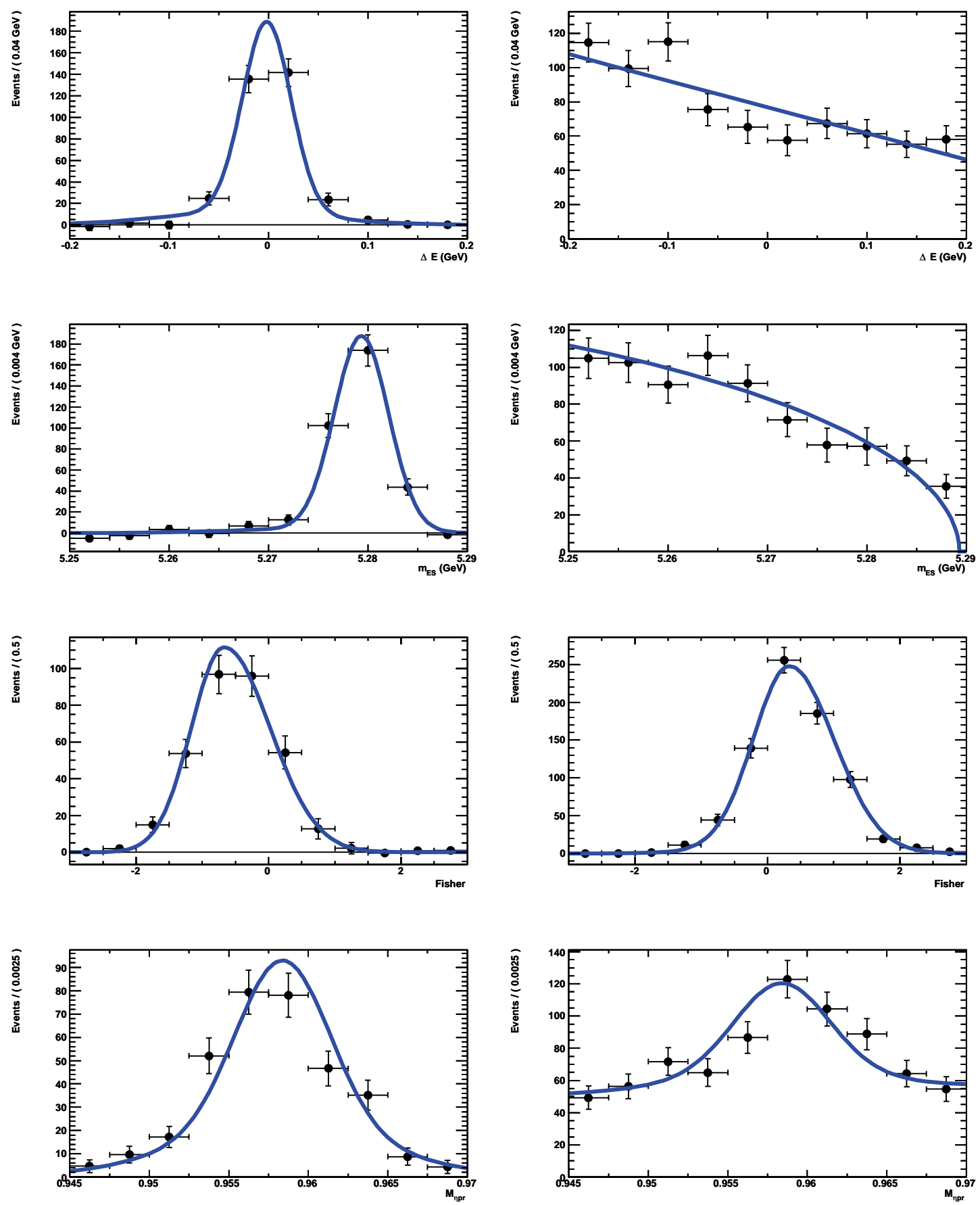

Figure 4.9: From top to bottom, the $B$ candidate $m_{\mathrm{ES}}, \Delta E, \mathcal{F}$, and $m_{\eta^{\prime}}$ sPlots for $\eta_{\eta \pi \pi}^{\prime} K_{S}^{0}$. The bottom row of plots is for information only and is not a variable used in the fit. The left column is signal and the right column is $q \bar{q}$ background. Signal yields are $333 \pm 22,331 \pm 21$, and $333 \pm 21$ when $m_{\mathrm{ES}}, \Delta E$, or $\mathcal{F}$ is removed from the fit. The nominal yield is $329 \pm 20$. 

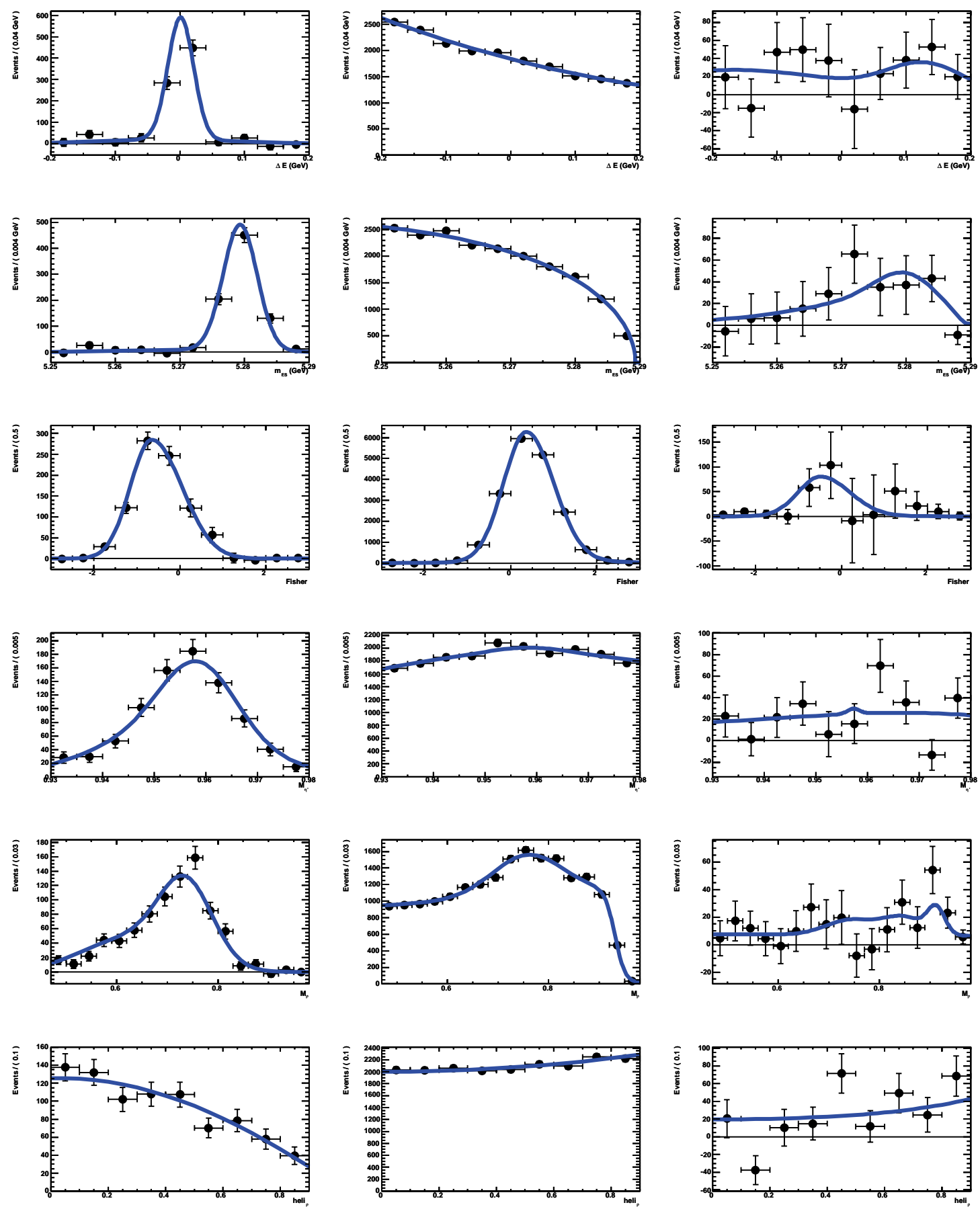

Figure 4.10: From top to bottom, the $B$ candidate $\Delta E, m_{\mathrm{ES}}, \mathcal{F}, m_{\eta^{\prime}}, m_{\rho}$, and $\mathcal{H}_{\rho}$ $s$ Plots for $\eta_{\rho \gamma}^{\prime} K_{S}^{0}$. The bottom three rows of plots are for information only and are not variables used in the fit. The left column is signal, middle column is $q \bar{q}$ background and the right column is charmless background. Signal yields are $849 \pm 48,829 \pm 67$, and $858 \pm 46$ when $m_{\mathrm{ES}}, \Delta E$, or $\mathcal{F}$ is removed from the fit. The nominal yield is $832 \pm 38$. 

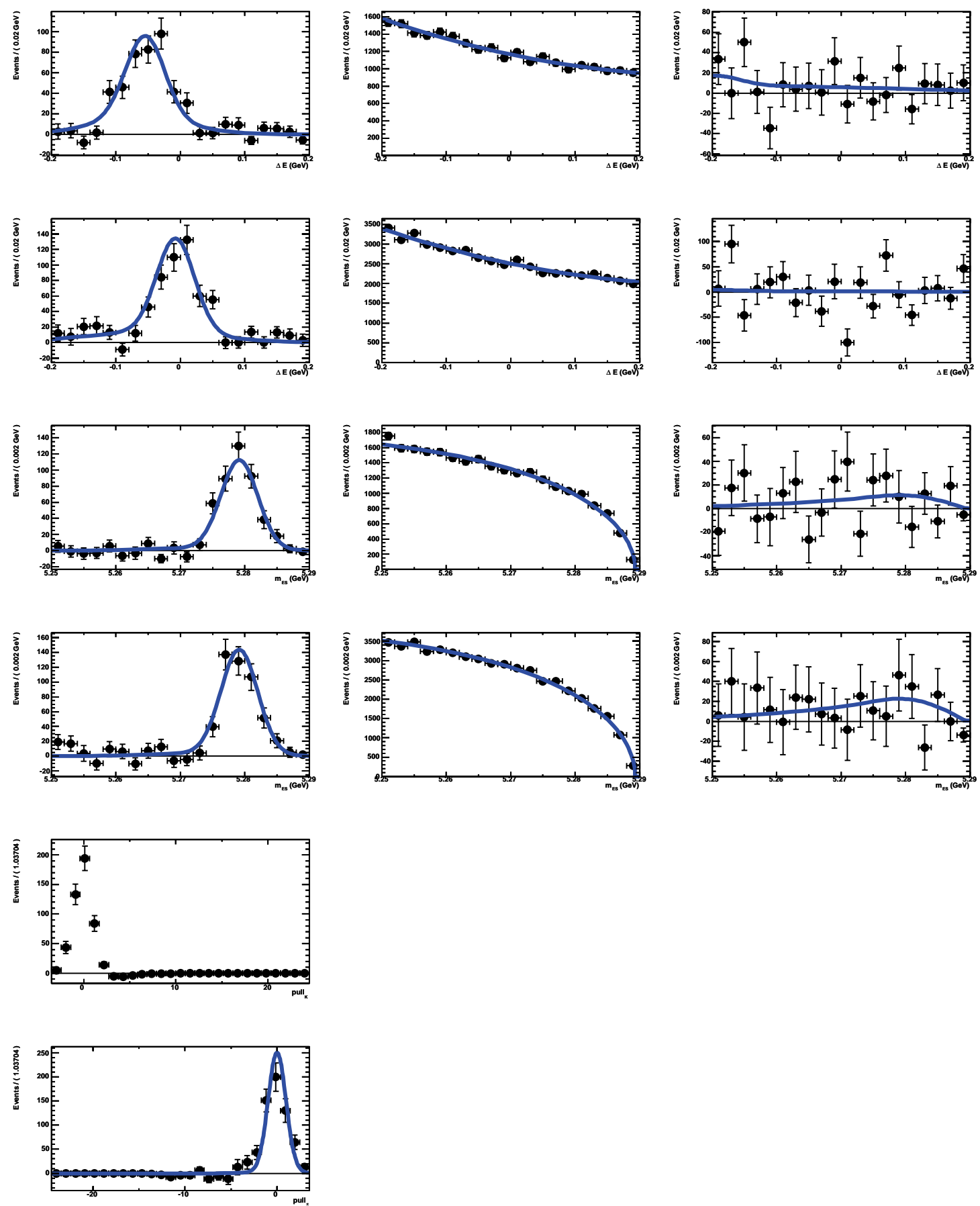

Figure 4.11: From top to bottom, the $B$ candidate $s$ Plots for $\Delta E, m_{\mathrm{ES}}$, and $S_{\text {pull }}$. The odd rows show $\omega \pi^{+}$and the even rows show $\omega K^{+}$. The left column is signal, middle column is $q \bar{q}$ background and the right column is charmless background. Signal yields for $\omega \pi^{+}$are $446 \pm 39,431 \pm 42$, and $382 \pm 54$ and for $\omega K^{+}$are $599 \pm 50,535 \pm 52$, and $582 \pm 56$ when $\Delta E, m_{\mathrm{ES}}$, or $S_{\text {pull }}$ is removed from the fit. The nominal yields are $457 \pm 32$ and $516 \pm 38$. 

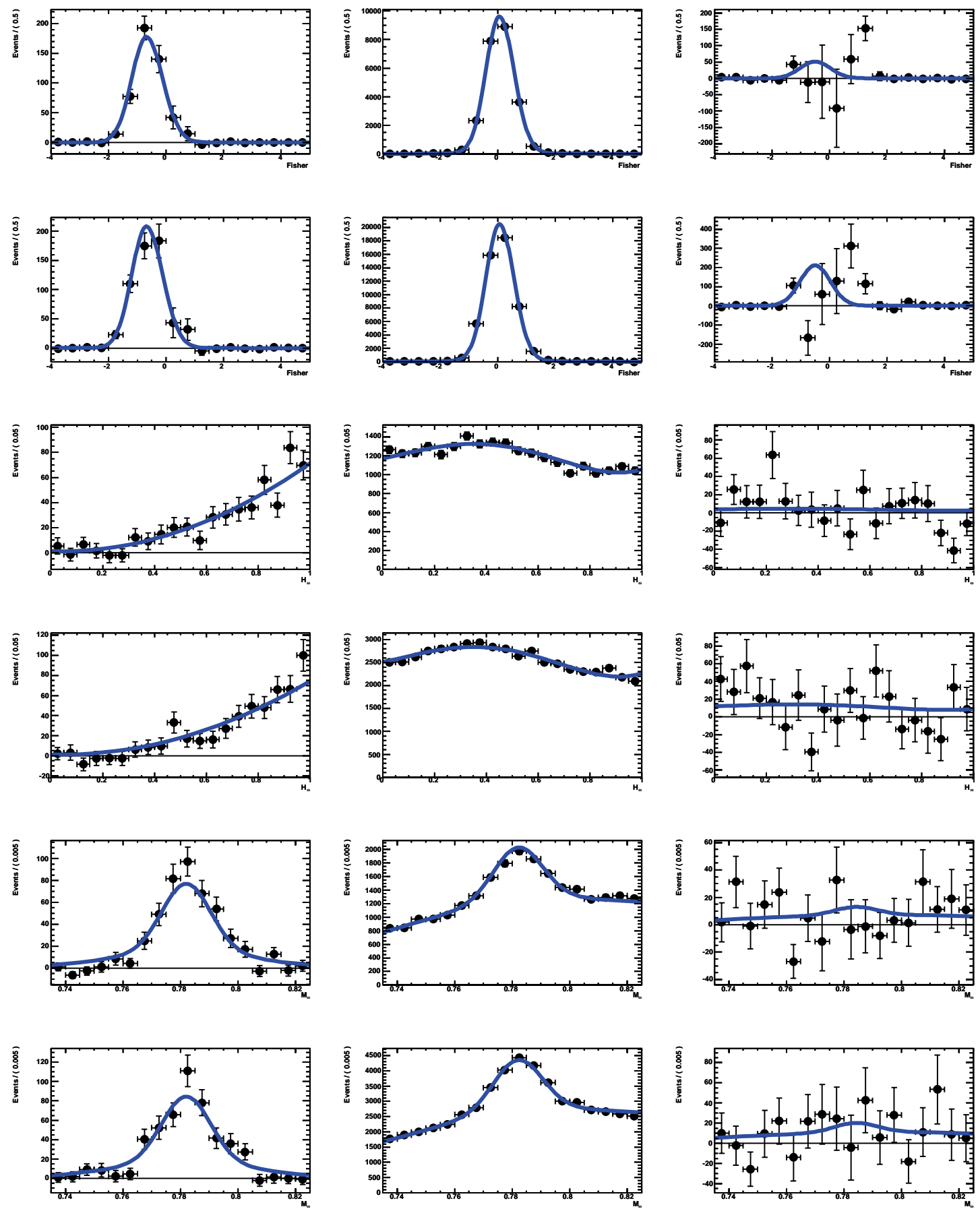

Figure 4.12: From top to bottom, the $B$ candidate $s$ Plots for $\mathrm{m}_{\omega}, \mathcal{H}_{\omega}$ and $\mathcal{F}$ sPlots. The odd rows show $\omega \pi^{+}$and the even rows show $\omega K^{+}$. The left column is signal, middle column is $q \bar{q}$ background and the right column is charmless background. Signal yields for $\omega \pi^{+}$are $438 \pm 34,478 \pm 37$, and $483 \pm 40$ and for $\omega K^{+}$are $471 \pm 38,487 \pm 43$, and $555 \pm 51$ when $\mathrm{m}_{\omega}, \mathcal{H}_{\omega}$ or $\mathcal{F}$ is removed from the fit. The nominal yields are $457 \pm 32$ and $516 \pm 38$. 

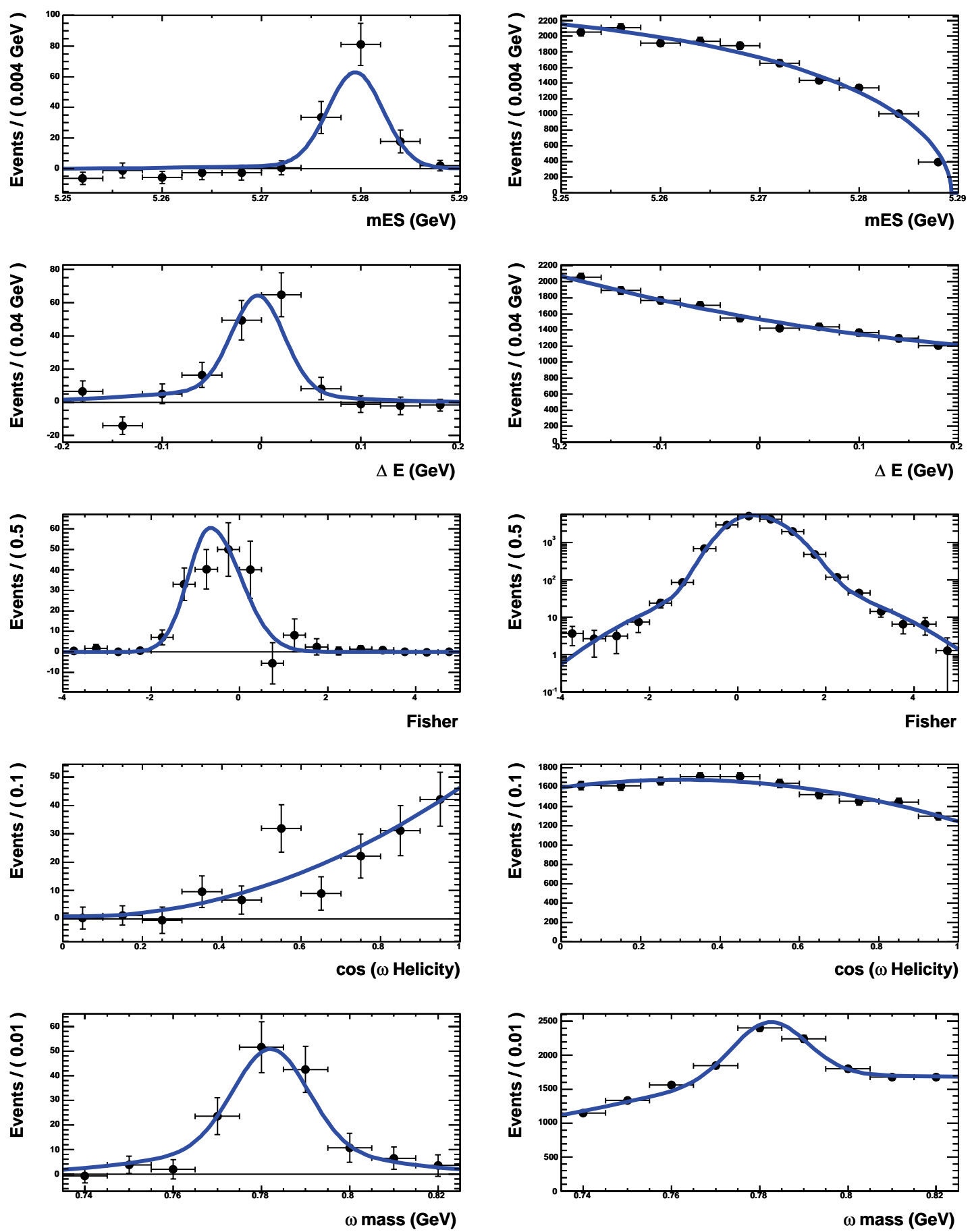

Figure 4.13: From top to bottom, the $B$ candidate $m_{\mathrm{ES}}, \Delta E, \mathcal{F}, \mathrm{m}_{\omega}$, and $\mathcal{H}_{\omega}$ sPlots for $\omega K_{S}^{0}$. The left column is signal and the right column is $q \bar{q}$ background. There are not $B \bar{B}$ plots because the yield is fixed in the fit. Signal yields are $115 \pm 22,130 \pm 21$, $179 \pm 27,142 \pm 19$ and $153 \pm 20$ when $m_{\mathrm{ES}}, \Delta E, \mathcal{F}, \mathrm{m}_{\omega}$, or $\mathcal{H}_{\omega}$ is removed from the fit. The nominal yield is $146 \pm 18$. 


\subsubsection{Likelihood Ratio Plots}

Figure 4.14 shows plots of the likelihood ratio $\mathcal{L}($ sig $) /[\mathcal{L}($ sig $)+\mathcal{L}(b k g)]$ for $\eta_{\eta \pi \pi}^{\prime} K^{+}$ and $\eta_{\rho \gamma}^{\prime} K^{+}$and Fig. 4.15 shows the likelihood ratios for $\eta_{\eta \pi \pi}^{\prime} K^{0}$ and $\eta_{\rho \gamma}^{\prime} K^{0}$. In these plots, the fit expectation from pure toy experiments is shown for signal plus background as the light-shaded region (green) and background alone as the dark-shaded region (red). The points show the data. A fit with signal events would show the data points in alignment with the signal plus background histogram, which extends above the background-only histogram near one. The good agreement between the data and the histogram indicates that the PDFs used in the likelihoods are a good representation of the data. In all cases clear signals are visible.

In Fig. 4.16 we show plots of the likelihood ratio $\mathcal{L}($ sig $) /[\mathcal{L}($ sig $)+\mathcal{L}(b k g)]$ for $\omega \pi^{+}$ and $\omega K^{+}$. The signal for $\omega K^{+}$is more peaked at high likelihood because the kaon track hypothesis has less background than the pion track hypothesis, despite total signals that are roughly equal. Figure 4.17 shows the likelihood ratio plot for $\omega K^{0}$. Again, signals are visible at high likelihoods, though less obvious than the $\eta^{\prime}$ channels where the signal yields are larger. 

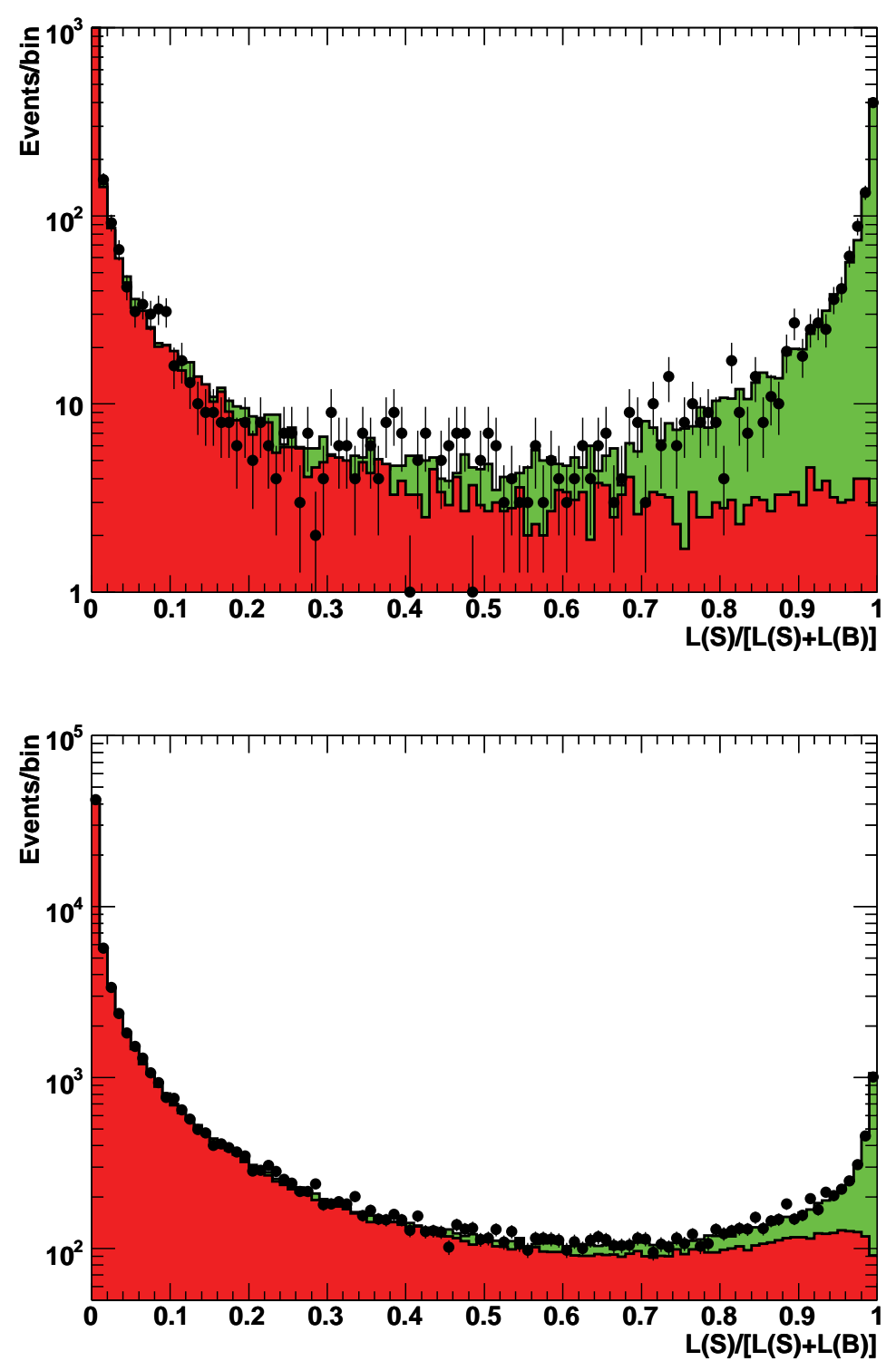

Figure 4.14: The likelihood ratio $\mathcal{L}(s i g) /[\mathcal{L}(s i g)+\mathcal{L}(b k g)]$ for $\eta_{\eta \pi \pi}^{\prime} K^{+}$(top) and $\eta_{\rho \gamma}^{\prime} K^{+}$ (bottom). The points represent the on-resonance data, the solid histograms are from pure toy samples of background plus charmless (red) and background plus charmless plus signal (green). 

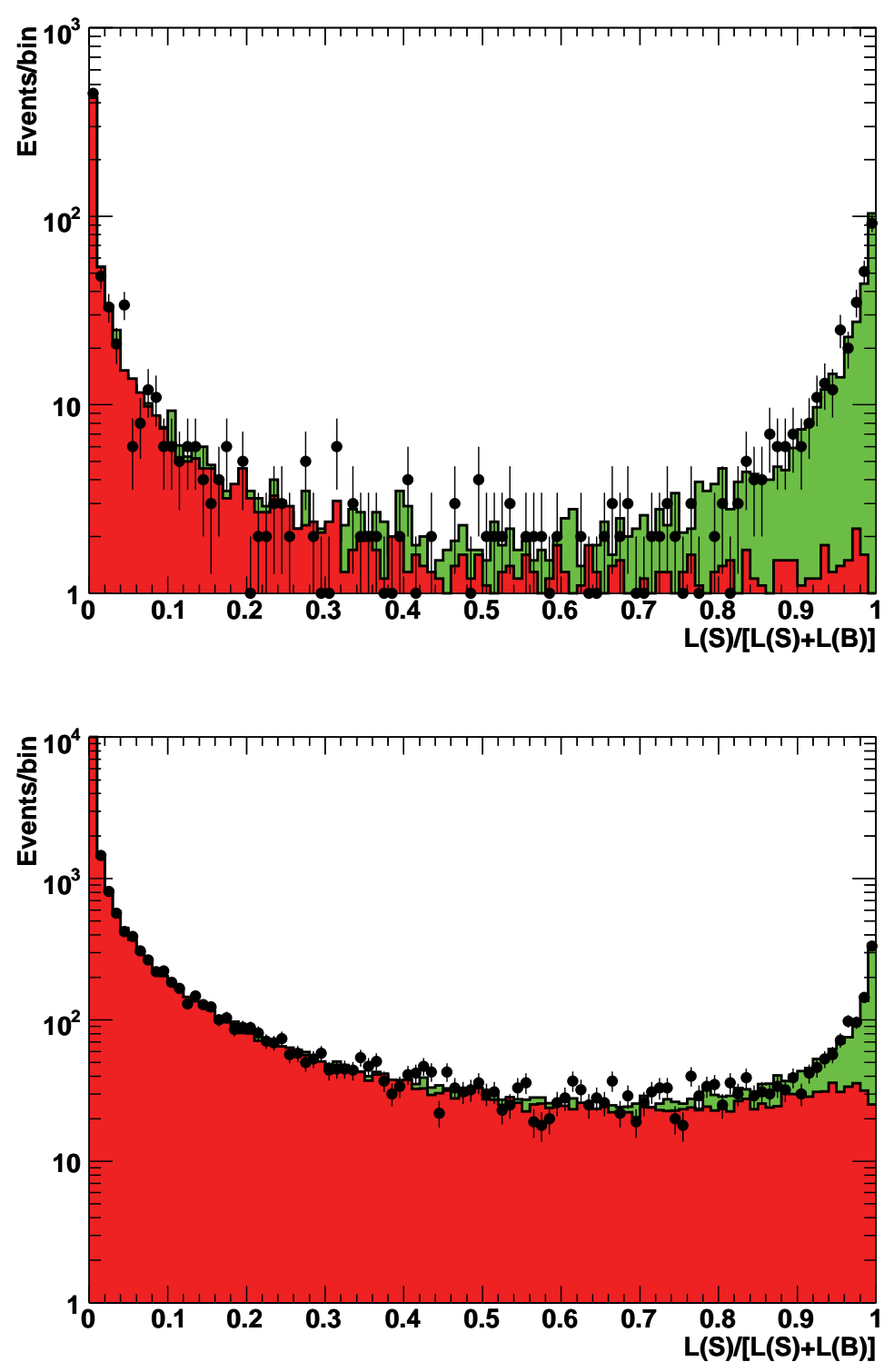

Figure 4.15: The likelihood ratio $\mathcal{L}(\operatorname{sig}) /[\mathcal{L}($ sig $)+\mathcal{L}(b k g)]$ for $\eta_{\eta \pi \pi}^{\prime} K^{0}$ (top) and $\eta_{\rho \gamma}^{\prime} K^{0}$ (bottom). The points represent the on-resonance data, the solid histograms are from pure toy samples of background plus charmless (red) and background plus charmless plus signal (green). 

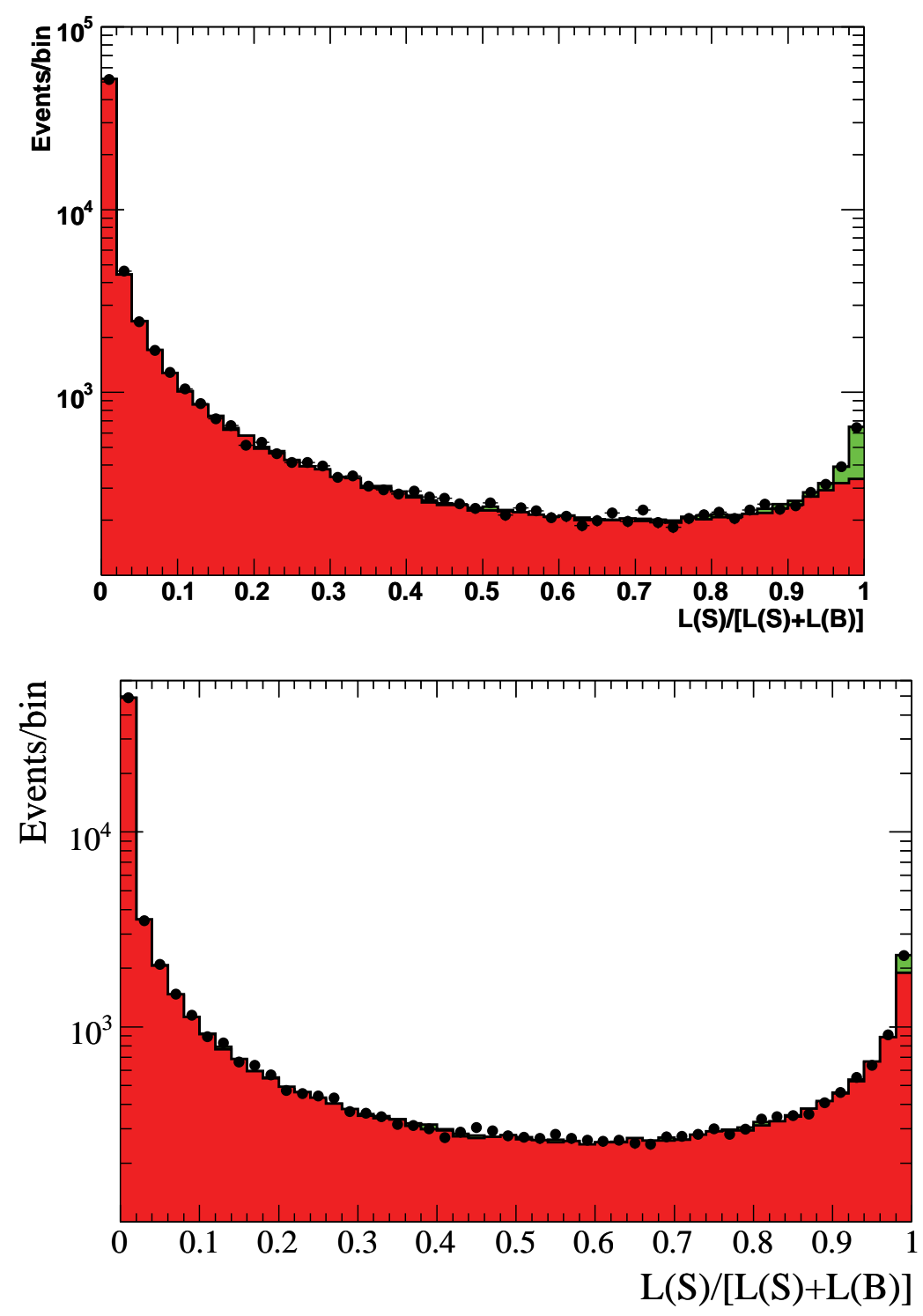

Figure 4.16: The likelihood ratio $\mathcal{L}(\operatorname{sig}) /[\mathcal{L}(s i g)+\mathcal{L}(b k g)]$ for $\omega \pi^{+}$(top) and $\omega K^{+}$ (bottom). The points represent the on-resonance data, the solid histograms are from pure toy samples of background plus charmless (red) and background plus charmless plus signal (green) for both modes fit together. 


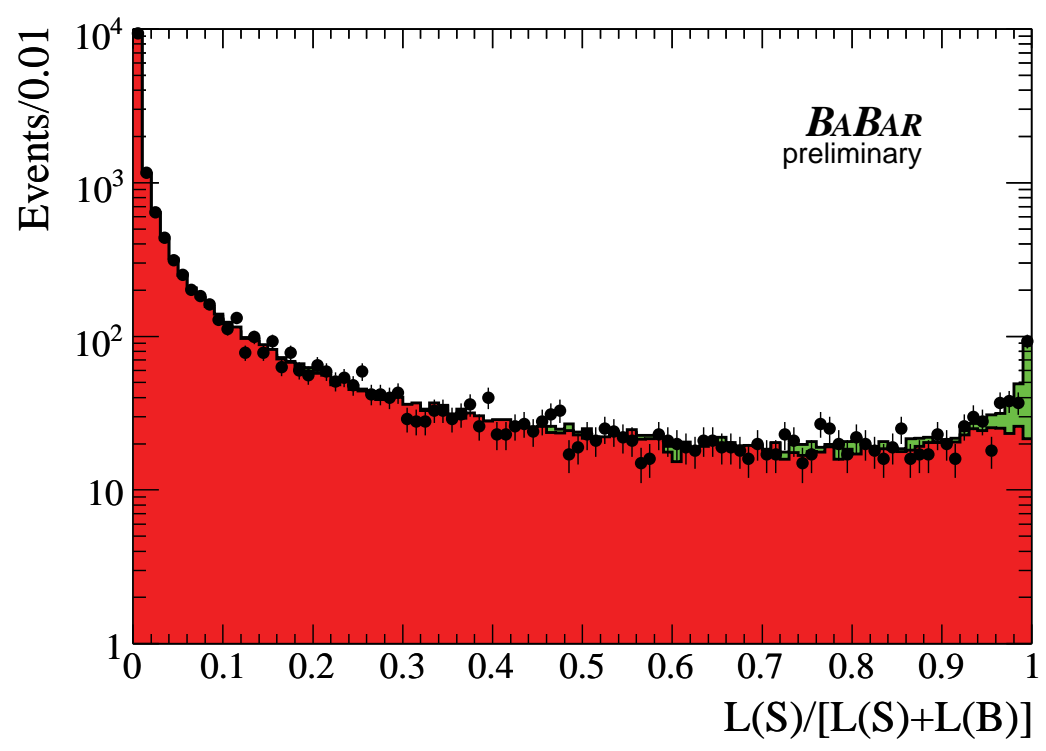

Figure 4.17: The likelihood ratio $\mathcal{L}($ sig $) /[\mathcal{L}(s i g)+\mathcal{L}(b k g)]$ for $\omega K^{0}$. The points represent the on-resonance data, the solid histograms are from pure toy samples of background plus charmless (red) and background plus charmless plus signal (green). 


\subsection{Time-dependent Results}

In this section I present the results of time-dependent $C P$ fits.

\subsection{1 $\quad C P$ Fits}

The $C P$ fit results for $\eta_{\eta \pi \pi}^{\prime} K^{0}, \eta_{\rho \gamma}^{\prime} K^{0}$, and $\omega K_{S}^{0}$ are shown in Table 4.3. No corrections have been applied to the results from the fitter.

Table 4.3: Results for the $B^{0} \rightarrow \eta^{\prime} K_{S}^{0}$ and $B^{0} \rightarrow \omega K_{S}^{0} \Delta t$ fits.

\begin{tabular}{lccc}
\hline \hline & $\eta_{\eta \pi \pi}^{\prime} K^{0}$ & $\eta_{\rho \gamma}^{\prime} K^{0}$ & $\omega K^{0}$ \\
\hline Events into fit & 1043 & 18839 & 13920 \\
\# Data combs/event & 1.08 & 1.08 & 1.13 \\
\# MC combs/event & 1.10 & 1.07 & 1.12 \\
Signal yield & $319 \pm 19$ & $789 \pm 36$ & $150_{-16}^{+17}$ \\
$B \bar{B}$ yield & - & $361 \pm 48$ & $36_{-22}^{+26}$ \\
$S$ & $0.61 \pm 0.23$ & $0.53 \pm 0.13$ & $0.63_{-0.28}^{+0.23}$ \\
$C$ & $-0.25 \pm 0.13$ & $-0.24 \pm 0.10$ & $-0.39_{-0.23}^{+0.24}$ \\
Combined fit: & & & \\
Signal yield & $295 \pm 19$ & $782 \pm 36$ & \\
$S$ & $0.55 \pm 0.12$ & \\
$C$ & \multicolumn{2}{c}{$0.24 \pm 0.08$} & \\
\hline \hline
\end{tabular}

\subsubsection{Discussion of $B \bar{B}$ Background}

We have conducted a toy study embedding different amounts of $B \bar{B}$ background to determine the effect on $S$ and $C$. Table 4.4 shows the yields and $S$ value embedding zero or the nominal number of $B \bar{B}$ events.

We find what appears to be a dilution bias in $S$ of about $2 \%$ that is associated with the $B \bar{B}$ background for the $\eta_{\rho \gamma}^{\prime} K^{0}$ decay. We find no significant sign of a bias in $S$ for $\eta^{\prime} \rightarrow \eta \pi^{+} \pi^{-}(S=0.692 \pm 0.007)$ and some indication of a bias in $S$ of $2-3 \%$ for $\eta^{\prime} \rightarrow \rho^{0} \gamma$, which is unrelated to the $B \bar{B}$ background. Any bias unrelated to $B \bar{B}$ background should be the same for $\eta^{\prime} \rightarrow \eta \pi^{+} \pi^{-}$and $\eta^{\prime} \rightarrow \rho^{0} \gamma$. We believe this 
Table 4.4: Results from toy studies for $\eta_{\rho \gamma}^{\prime} K^{0} \Delta t$ fits embedding 770 signal events and 360 or $0 B \bar{B}$ events as shown in the table. In each case 1000 experiments were fit.

\begin{tabular}{ccccr}
\hline \hline $\begin{array}{c}\# B \bar{B} \\
\text { embed }\end{array}$ & $\begin{array}{c}\text { Signal } \\
\text { bias }\end{array}$ & $\begin{array}{c}B \bar{B} \\
\text { yield }\end{array}$ & $S$ & \multicolumn{1}{c}{$C$} \\
\hline 0 & $10.8 \pm 0.7$ & $-11.0 \pm 1.6$ & $0.680 \pm 0.004$ & $-0.008 \pm 0.003$ \\
360 & $20.0 \pm 0.7$ & $291.0 \pm 1.5$ & $0.670 \pm 0.004$ & $0.000 \pm 0.003$ \\
\hline \hline
\end{tabular}

bias is at least partially a statistical fluctuation. We correct for the bias due to $B \bar{B}$ $\left(+0.01\right.$ in $\left.\eta_{\rho \gamma}^{\prime} K^{0}\right)$ and assign a systematic for this correction (0.01) and for the statistical uncertainty on these studies (0.01).

A similar toy study was performed for the $B \bar{B}$ events in $B^{0} \rightarrow \omega K_{S}^{0}$. In Table 4.5 we show a set of toy experiments embedding $0 B \bar{B}$ events compared with the nominal result with $35 B \bar{B}$ events embedded. Less than one event is found to enter into the signal from the $B \bar{B}$, so the different values for $S$ must be statistical fluctuation.

Table 4.5: Summary of toy embedding studies for $\omega K_{S}^{0} \Delta t$ fits. Results are shown for various numbers of embedded charmless events. In each case 143 signal events are embedded and 3000 toy experiments were run.

\begin{tabular}{|c|c|c|c|c|}
\hline $\begin{array}{l}\# B \bar{B} \\
\text { embed }\end{array}$ & $\begin{array}{c}\text { Signal } \\
\text { bias }\end{array}$ & $\begin{array}{c}B \bar{B} \\
\text { yield }\end{array}$ & $S$ & $C$ \\
\hline 0 & $6.3 \pm 0.2$ & $-19.6 \pm 0.5$ & $0.697 \pm 0.007$ & $-0.003 \pm 0.005$ \\
\hline 35 & $6.8 \pm 0.2$ & $14.4 \pm 0.5$ & $0.715 \pm 0.007$ & $-0.003 \pm 0.005$ \\
\hline
\end{tabular}

\subsubsection{Pull Widths and Underestimated Errors}

In the previous version of this analysis we found that the errors of the fit for the time-dependent asymmetries can be underestimated for small signal yields. The effect is worse near the physical boundary of $S, C= \pm 1$. Small signal yields result in large 
uncertainties, so even for central values near 0 , it is possible to observe this boundary effect. To account for this effect, we apply a scale factor to the $\omega K_{S}^{0}$ errors based on the width of the pull distribution from toy experiments. Because the effect is statistical and related to the mean value of the distribution, we take the final scale factors from pure toy experiments where we are able to generate and fit the experiments using our fit values of $S$ and $C$. From pure toy experiments (in Sec.3.10.3.1) we find a pull distribution width of $S=1.06$ and $C=1.03$, which we use to scale the fit uncertainties. Figure 4.18 shows the error distribution from embedded toys with our scaled fit error based on the widths quoted above marked in the plot. The scaled errors agree well with the expectation from the toys and are quoted as the final result.
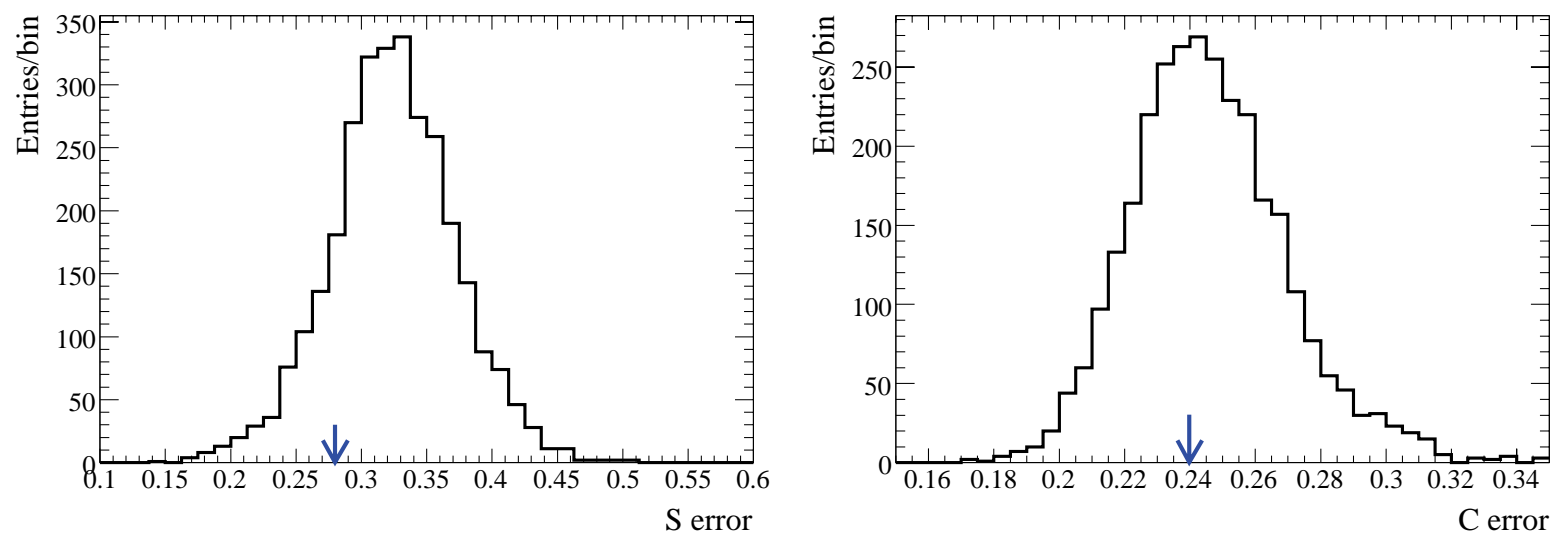

Figure 4.18: Error distributions for embedded toy experiments for $B^{0} \rightarrow \omega K^{0}$ for $S$ (left) and $C$ (right). The arrows show the scaled error value from the fit.

The signal yields in the $\eta_{\rho \gamma}^{\prime} K^{0}$ and $\eta_{\eta \pi \pi}^{\prime} K^{0}$ channels are larger and the uncertainties on $S$ and $C$ are smaller, so no such underestimated error effect is expected in these channels. The pulls of the toy distributions for $S$ are shown in Fig. 4.19. We find that the width of the distribution for $\eta_{\rho \gamma}^{\prime} K^{0}$ and $\eta_{\eta \pi \pi}^{\prime} K^{0}$ are both consistent with one indicating that no scale factor is needed. 

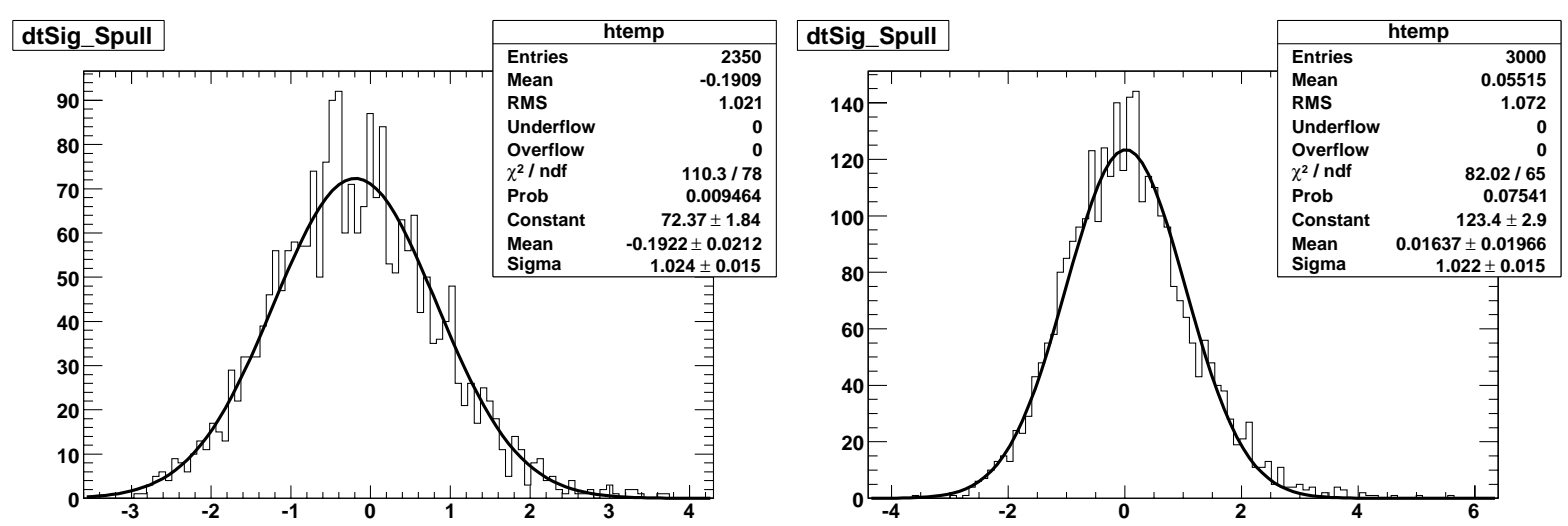

Figure 4.19: The pull distribution for $S$ for $\eta_{\rho \gamma}^{\prime} K^{0}$ (left) and $\eta_{\eta \pi \pi}^{\prime} K^{0}$ (right) from embedded toy experiments.

\subsubsection{Projection Plots}

In Fig. 4.20 we show projections of the $\eta_{\rho \gamma}^{\prime} K^{0}$ and $\eta_{\eta \pi \pi}^{\prime} K^{0}$ decay modes onto $m_{\mathrm{ES}}$ and $\Delta E$ for a subset of the data for which the signal likelihood (computed without the variable plotted) exceeds a mode-dependent threshold that optimizes the sensitivity for the time-dependent fits.

In Fig. 4.21 we show the $\Delta t$ projections and raw asymmetry for the combined $B^{0} \rightarrow \eta^{\prime} K_{S}^{0}$ modes that are made by applying a cut on event likelihood.

Figure 4.22 shows the projections of $\omega K_{S}^{0}$ onto $m_{\mathrm{ES}}, \Delta E, \mathcal{F}, \mathrm{m}_{\omega}$, and $\mathcal{H}_{\omega}$, also with the mode-dependent threshold requirement. The fraction of signal included in the plots is between $55 \%$ and $85 \%$. The signal likelihood ratio requirements are between $0.90-0.98$. In Fig. 4.23 we show the $\Delta t$ projections and raw asymmetry for $B^{0} \rightarrow \omega K^{0}$, which are also produced with the requirement on signal event likelihood.

\subsection{5 sPlots}

In Fig. 4.24 we show sPlots of the signal and $q \bar{q}$ background components of $\Delta E$, $m_{\mathrm{ES}}, \mathcal{F}, \Delta t$, and $m_{\eta^{\prime}}$, for $\eta_{\eta \pi \pi}^{\prime} K^{0}$. Figure 4.25 shows the $s$ Plots of $\Delta E, m_{\mathrm{ES}}, \mathcal{F}, \mathrm{m}_{\eta^{\prime}}$, $\mathrm{m}_{\rho}, \mathcal{H}_{\rho}$, and $\Delta t$ for $\eta_{\rho \gamma}^{\prime} K^{0}$ for signal, $q \bar{q}$ background and charmless background. In 

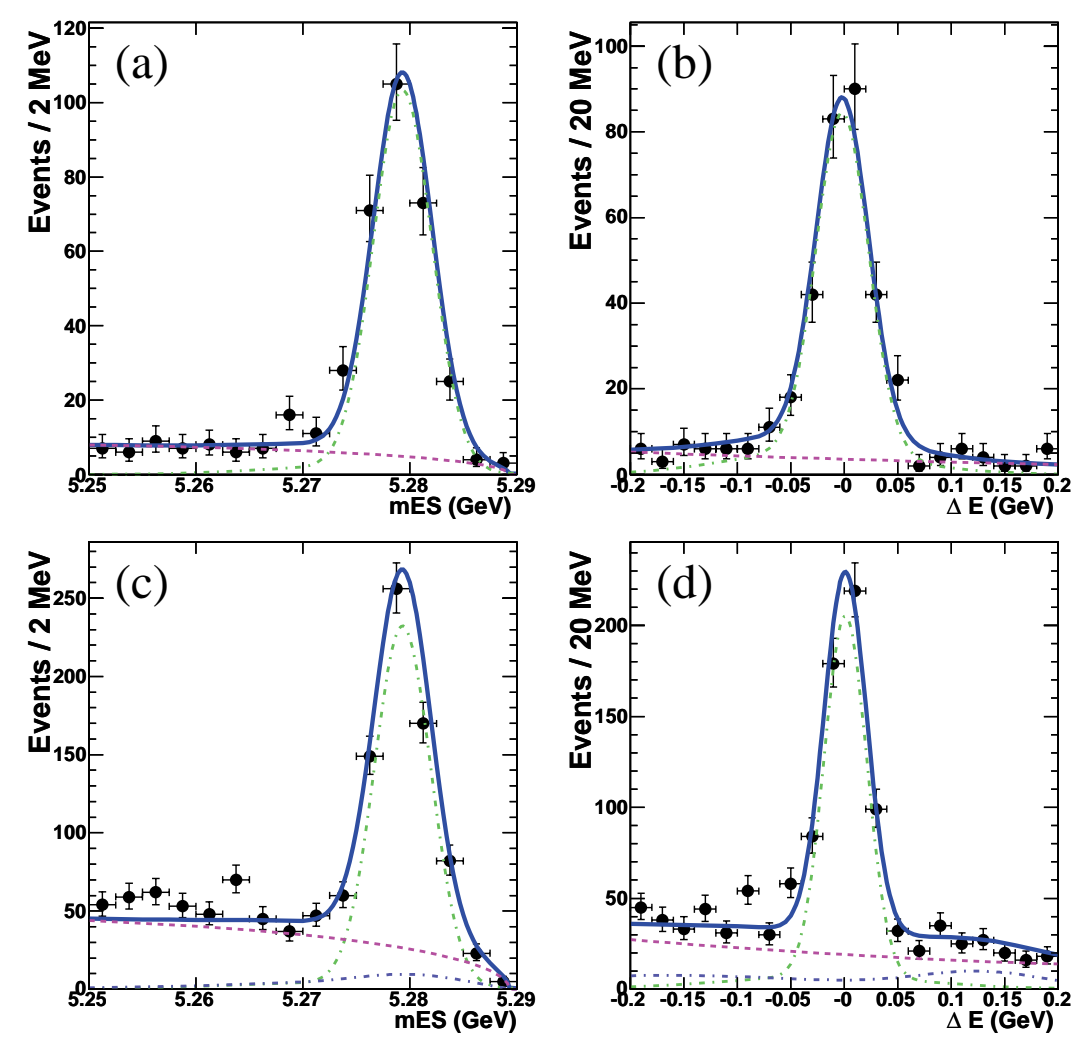

Figure 4.20: The $B$ candidate $m_{\mathrm{ES}}$ and $\Delta E$ projections for $(\mathrm{a}, \mathrm{b}) B^{0} \rightarrow \eta_{\eta \pi \pi}^{\prime} K^{0}$ and (c, d) $B^{0} \rightarrow \eta_{\rho \gamma}^{\prime} K^{0}$. Points with errors represent the data, solid curves the full fit functions, and dashed curves the background functions. 


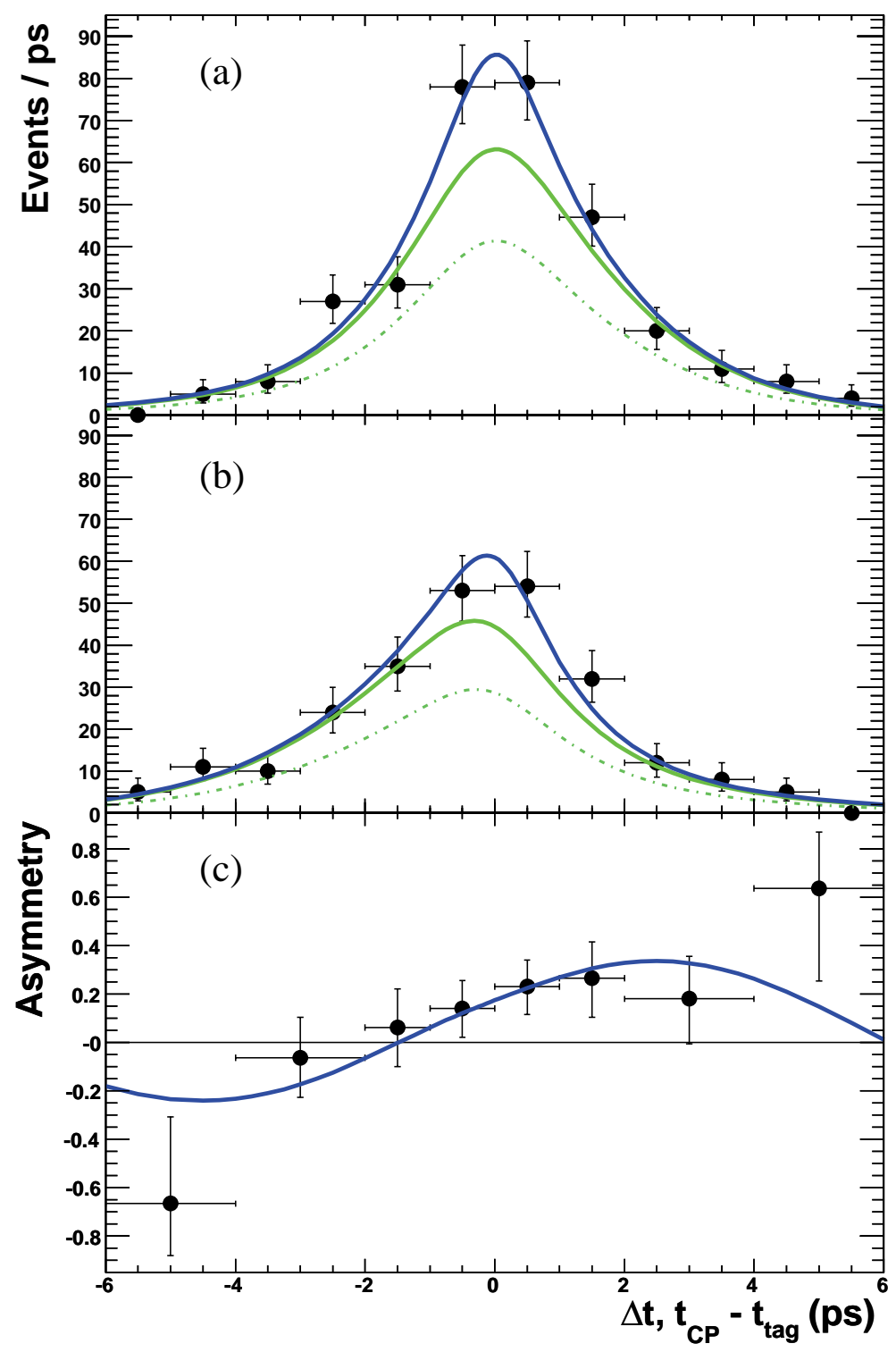

Figure 4.21: Projections onto $\Delta t$ for data for both submodes combined (points with errors), the fit function for both submodes combined (blue solid line), the fit function for $\eta_{\rho \gamma}^{\prime} K^{0}$ only (green solid line) and the signal only function for $\eta_{\rho \gamma}^{\prime} K^{0}$ (green dashed line). We show (a) $B^{0}$ and (b) $\bar{B}^{0}$ tagged events, and (c) the asymmetry between $B^{0}$ and $\bar{B}^{0}$ tags. 

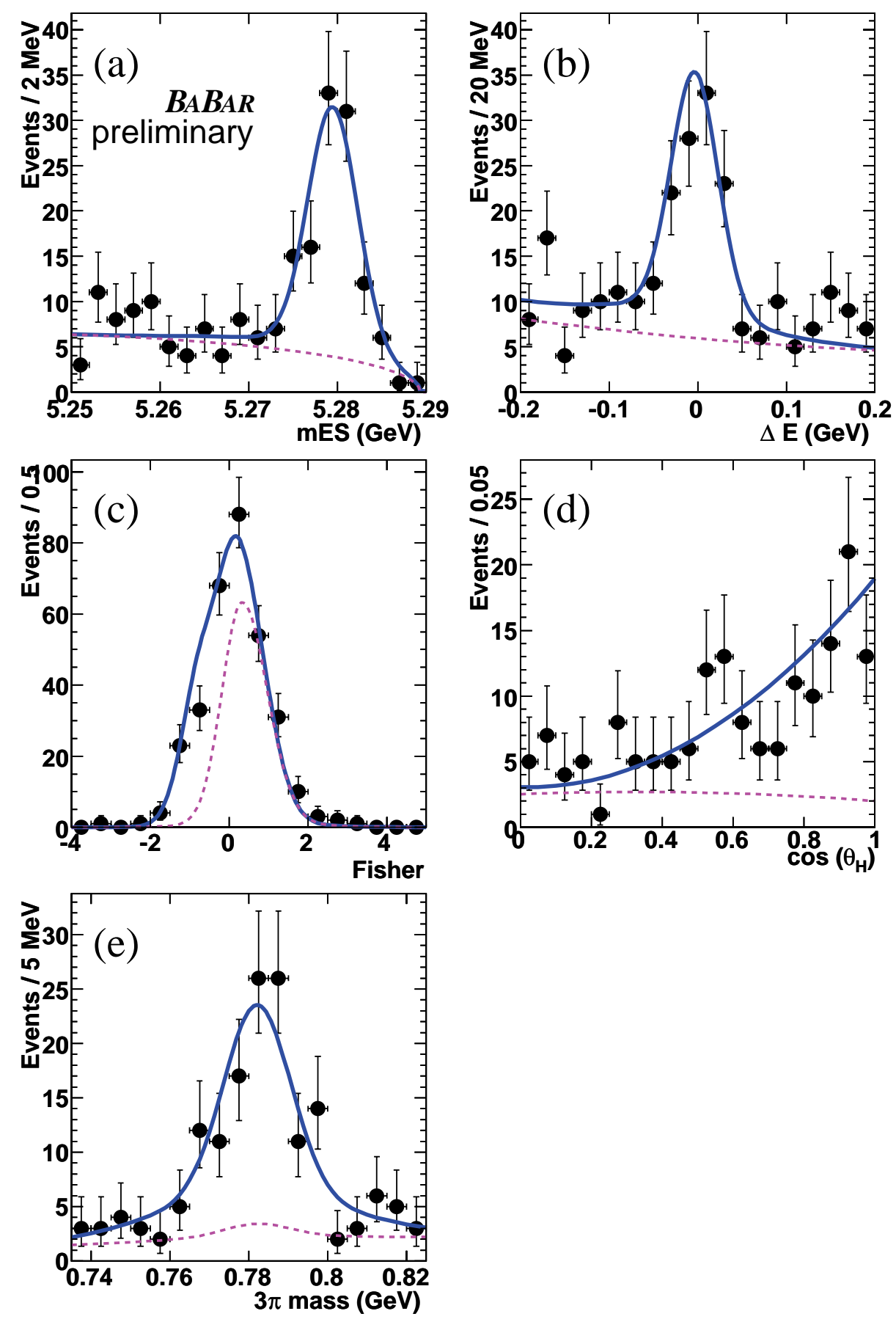

Figure 4.22: Projection plots for (a) $m_{\mathrm{ES}}$, (b) $\Delta E$, (c) $\mathcal{F}$, (d) $\mathcal{H}_{\omega}$, and (e) $\mathrm{m}_{\omega}$ for $\omega K^{0}$ showing data (points with errors), the fit function (solid line) and background function (dashed line). 


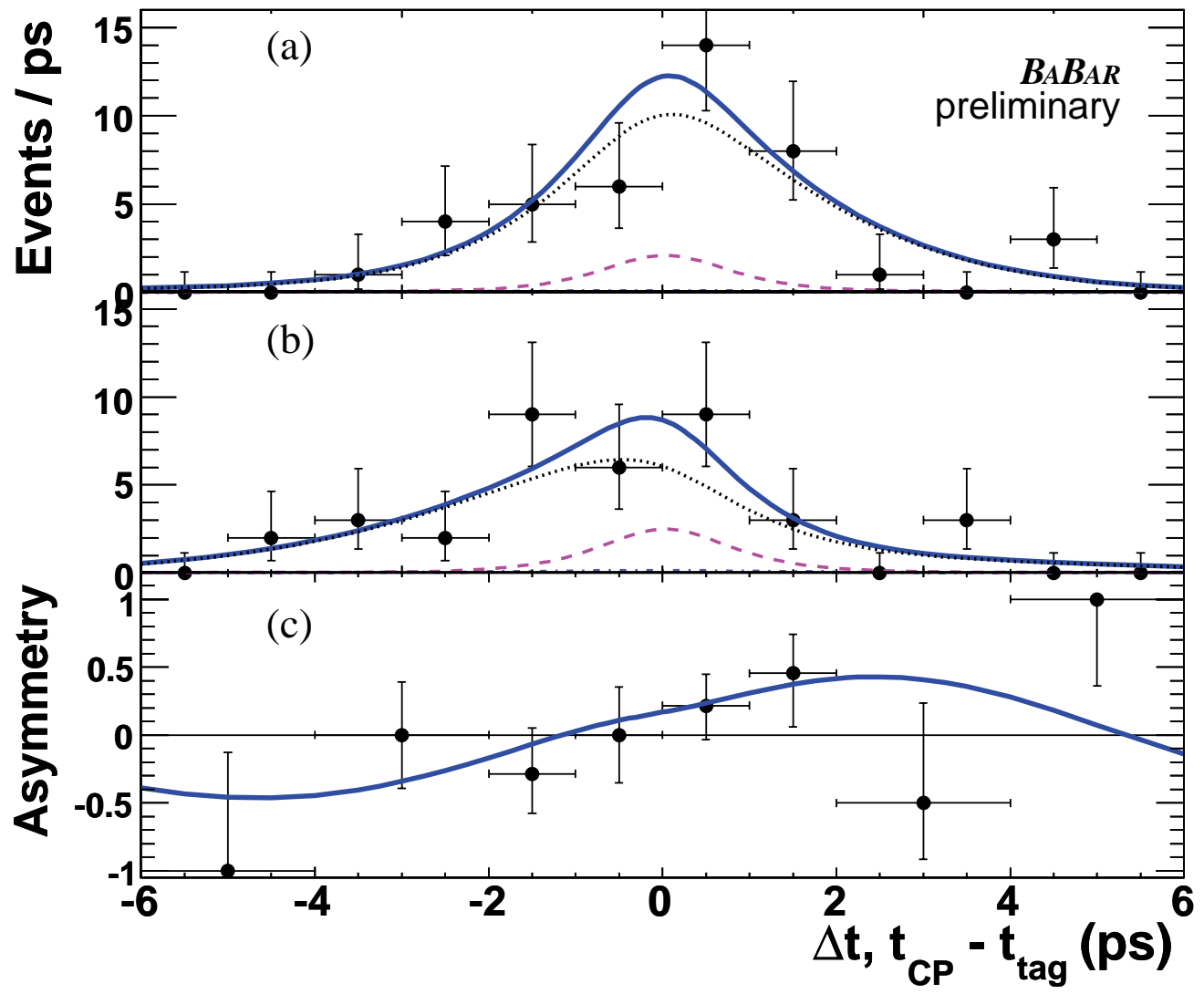

Figure 4.23: Projections onto $\Delta t$ for $B^{0} \rightarrow \omega K^{0}$. Data (points with errors), the fit function (solid line), signal function (dotted line) and background function (dashed line), for (a) $B^{0}$ and (b) $\bar{B}^{0}$ tagged events, and (c) the asymmetry between $B^{0}$ and $\bar{B}^{0}$ tags. 
both cases plots are shown for variables not in the fit to give confidence that the signal and backgrounds are well understood in all variables. The good agreement between the PDF curves and sPlot data gives confidence in the performance of the time-dependent fits.

Figure 4.26 shows sPlots of the signal, $q \bar{q}$ background, and $B \bar{B}$ background components of $m_{\mathrm{ES}}, \Delta E, \mathrm{~m}_{\omega}, \mathcal{H}_{\omega}$, and $\mathcal{F}$ for $\omega K_{S}^{0}$. The sPlots of $\mathcal{F}$ reveal an excess of events in the charmless yield. The plot shows that these events are actually $q \bar{q}$-like in $\mathcal{F}$. We find that the charmless yield goes from $36 \pm 24$ to $82 \pm 43$ when $\mathcal{F}$ is removed from the fit, as is done in making the $\mathcal{F}$ sPlot. The signal yield goes from $150 \pm 16$ to $173 \pm 21$. The increased errors suggest there is simply not enough discriminating power to effectively distinguish between $q \bar{q}$ and charmless events without $\mathcal{F}$ in the fit.

\subsubsection{Likelihood Plots}

Figure 4.27 shows the likelihood plots comparing data with toys produced with and without signal events for the $\eta^{\prime}$ modes. Figure 4.28 shows the likelihood plot for the $\omega K_{S}^{0}$ time-dependent fit. In all cases the signals appear as an excess above the background, in good agreement with the expectations from toys.

\subsubsection{Combining NLL Plots}

In Fig. 4.29 we show the likelihood curves as a function of $S$ and $C$ for both $\eta_{\rho \gamma}^{\prime} K^{0}$ and $\eta_{\eta \pi \pi}^{\prime} K^{0}$ along with the combined plots. The combined results can be calculated in two ways: a combined fit to the submodes as shown in Table 4.3 or combining the NLL curves from fits to the individual modes as documented in App. F. We use the combined curve method because of it's ability to include the systematic errors in the combining. The two methods agree well as shown in Table 4.6. 

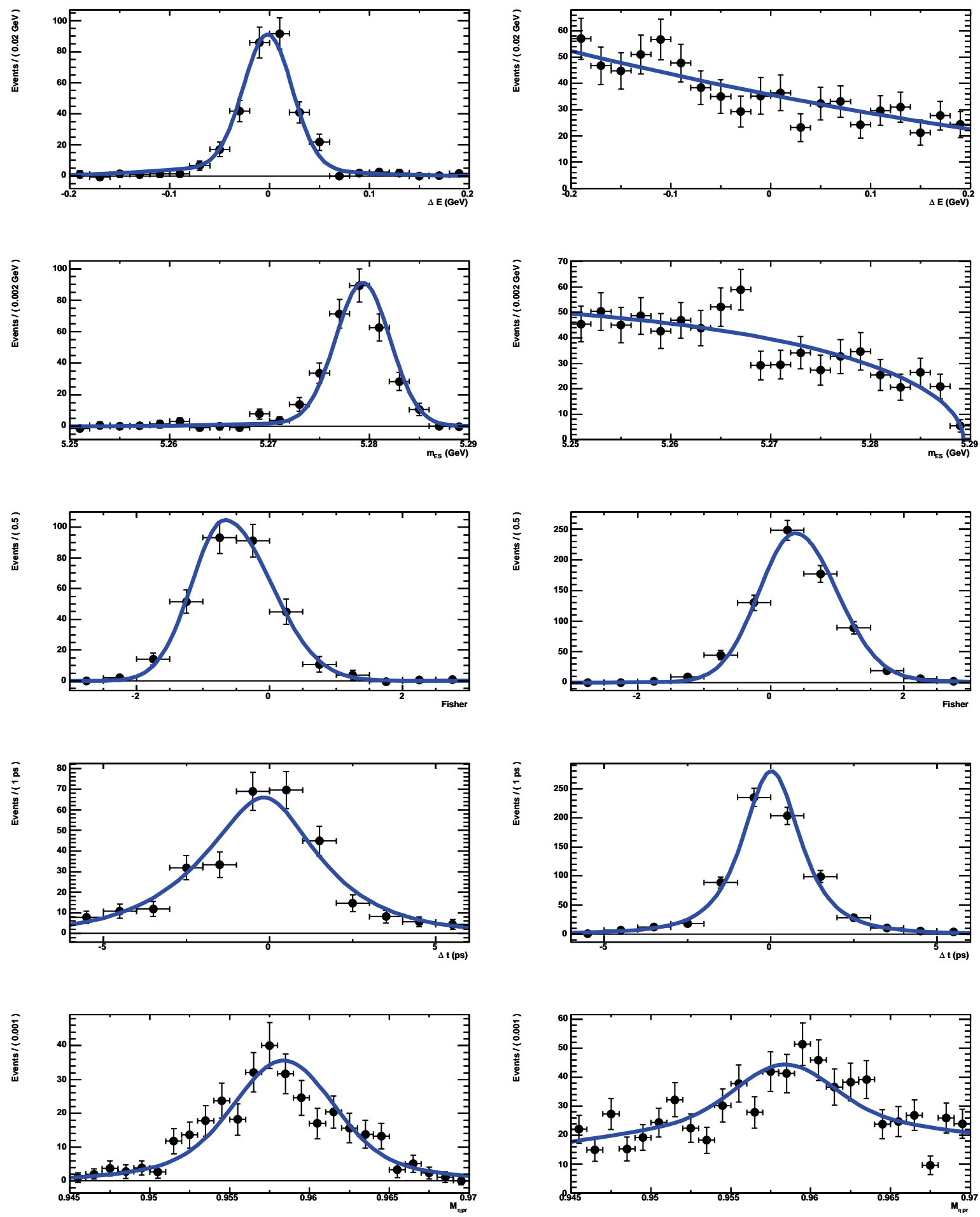

Figure 4.24: From top to bottom, the $B$ candidate $\Delta E, m_{\mathrm{ES}}, \mathcal{F}, \Delta t$, and $m_{\eta^{\prime}}$ sPlots for $\eta_{\eta \pi \pi}^{\prime} K^{0}$. The bottom row of plots is for information only and is not a variable used in the fit. The left column is signal and the right column is background. 

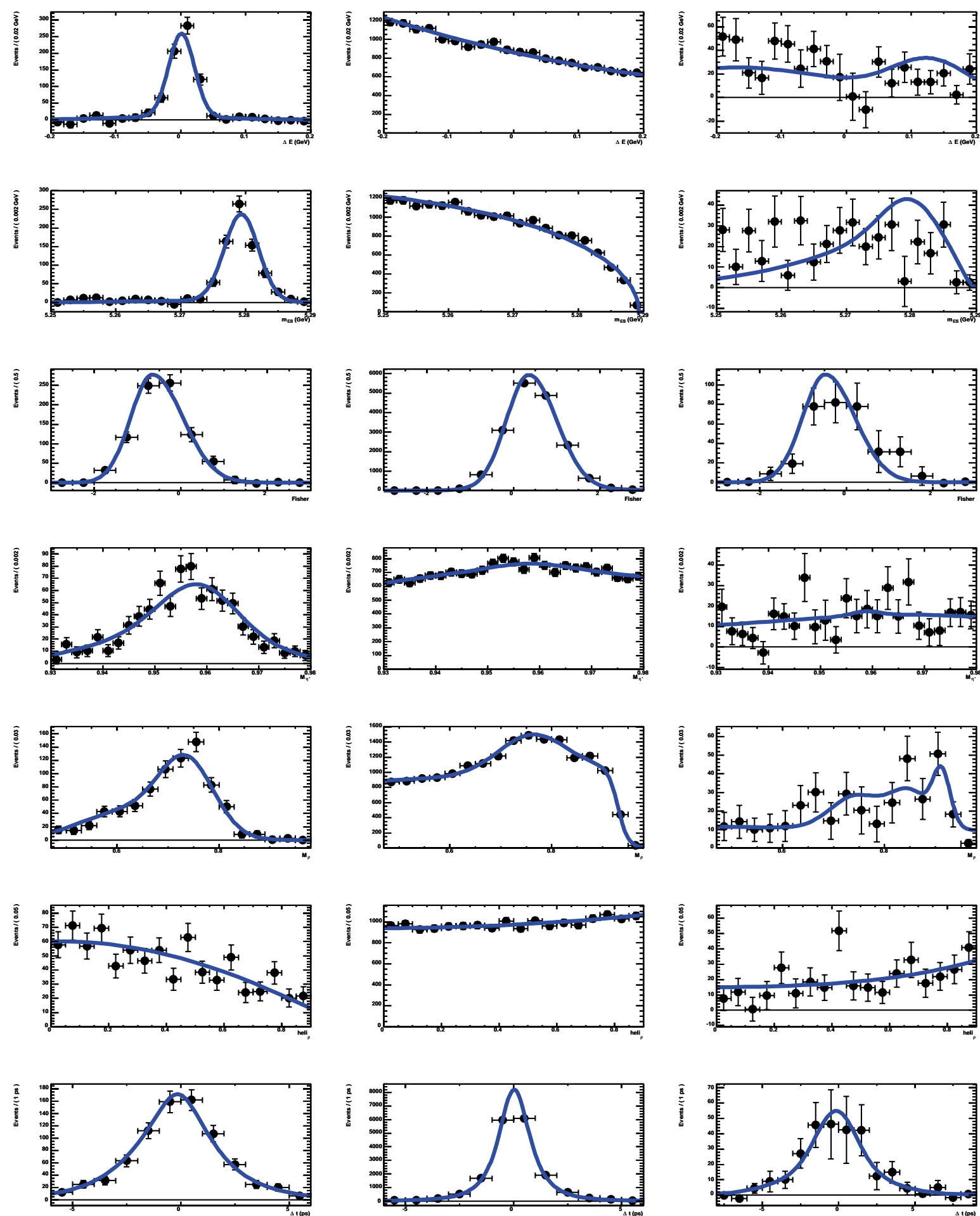

Figure 4.25: From top to bottom, the $B$ candidate $\Delta E, m_{\mathrm{ES}}, \mathcal{F}, \mathrm{m}_{\eta^{\prime}}, \mathrm{m}_{\rho}, \mathcal{H}_{\rho}$, and $\Delta t$ $s$ Plots for $\eta_{\rho \gamma}^{\prime} K^{0}$. The fourth, fifth, and sixth rows are for information only and are not variables used in the fit. The left column is signal, the middle column is background, and the right column is $B \bar{B}$. 

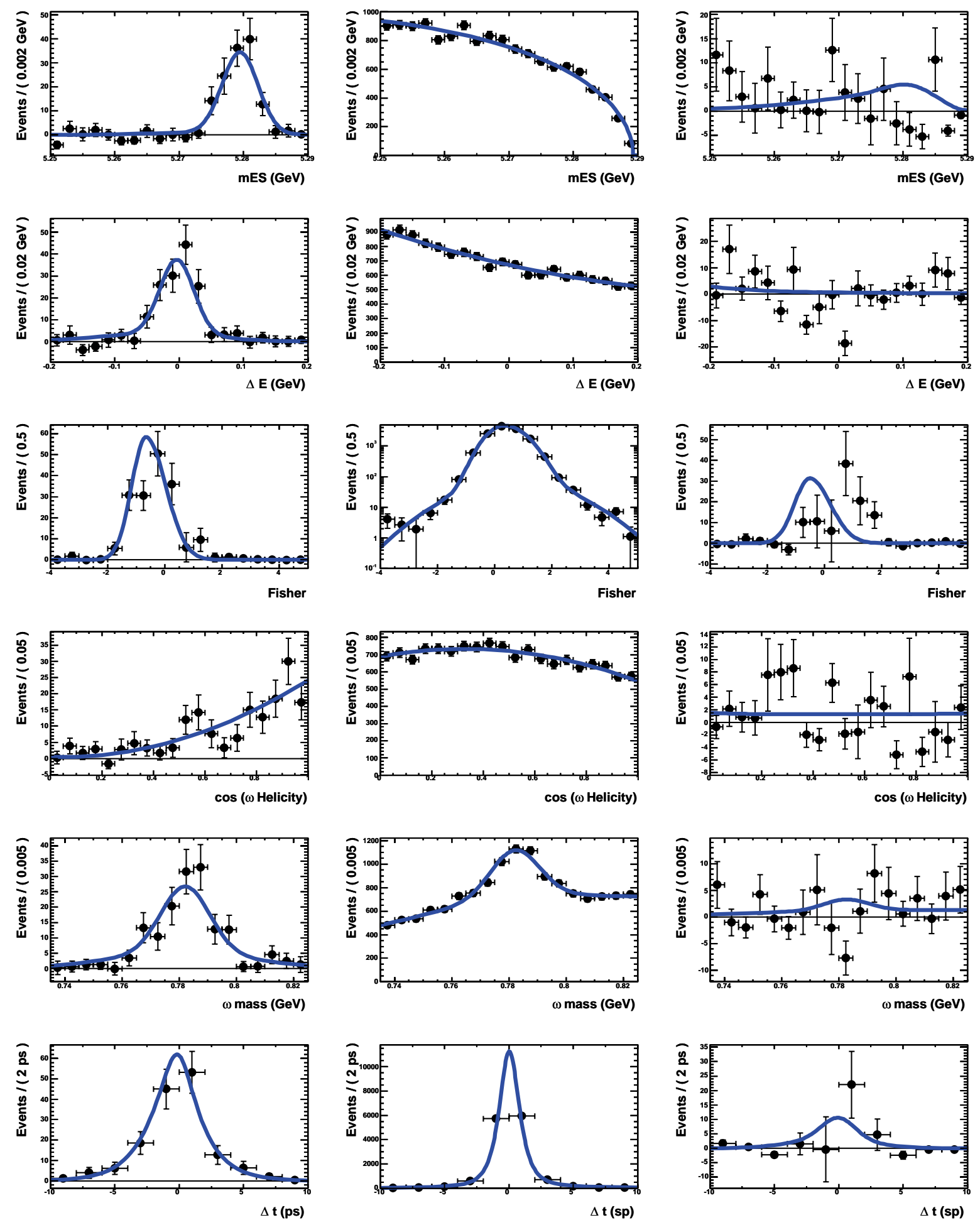

Figure 4.26: From top to bottom, the $B$ candidate $m_{\mathrm{ES}}, \Delta E, \mathrm{~m}_{\omega}, \mathcal{H}_{\omega}$, and $\mathcal{F}$ sPlots for $\omega K_{S}^{0}$. The left column is signal, the middle column is background, and the right column is $B \bar{B}$. 

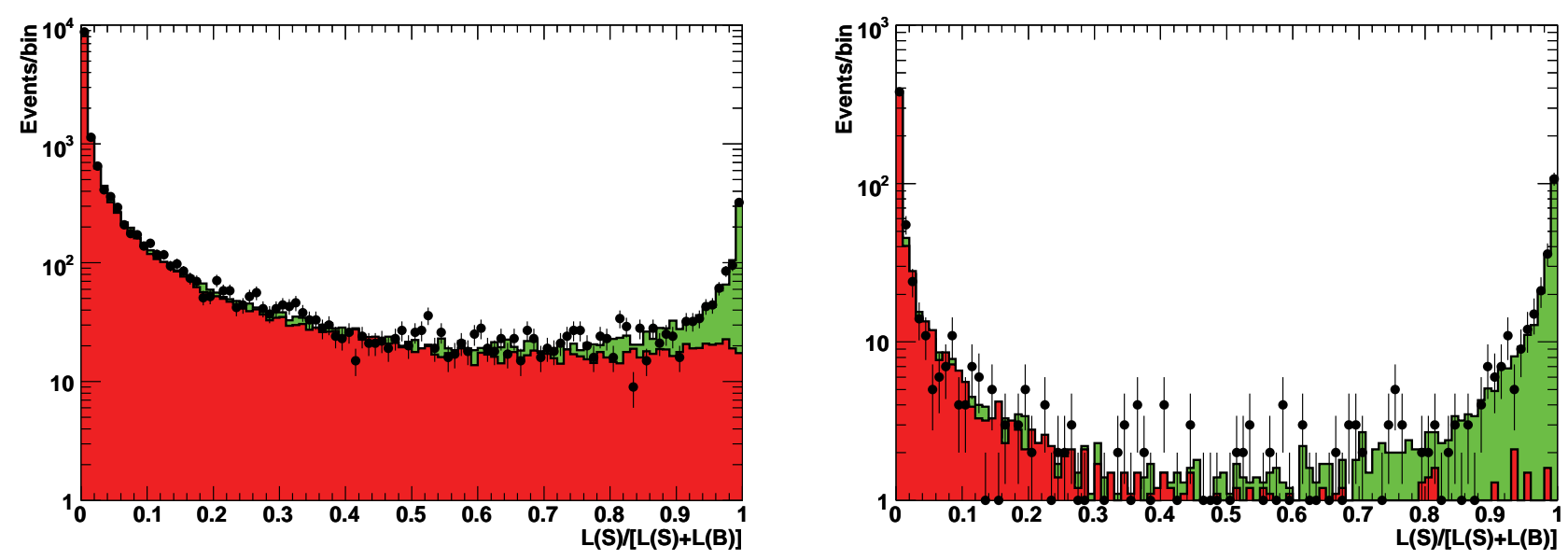

Figure 4.27: The likelihood ratio $\mathcal{L}(s i g) /[\mathcal{L}(s i g)+\mathcal{L}(b k g)]$ for $\eta_{\rho \gamma}^{\prime} K^{0}$ (left) and $\eta_{\eta \pi \pi}^{\prime} K^{0}$ (right). The points represent the on-resonance data, the solid histograms are from pure toy samples of background (red), and background plus signal (green).

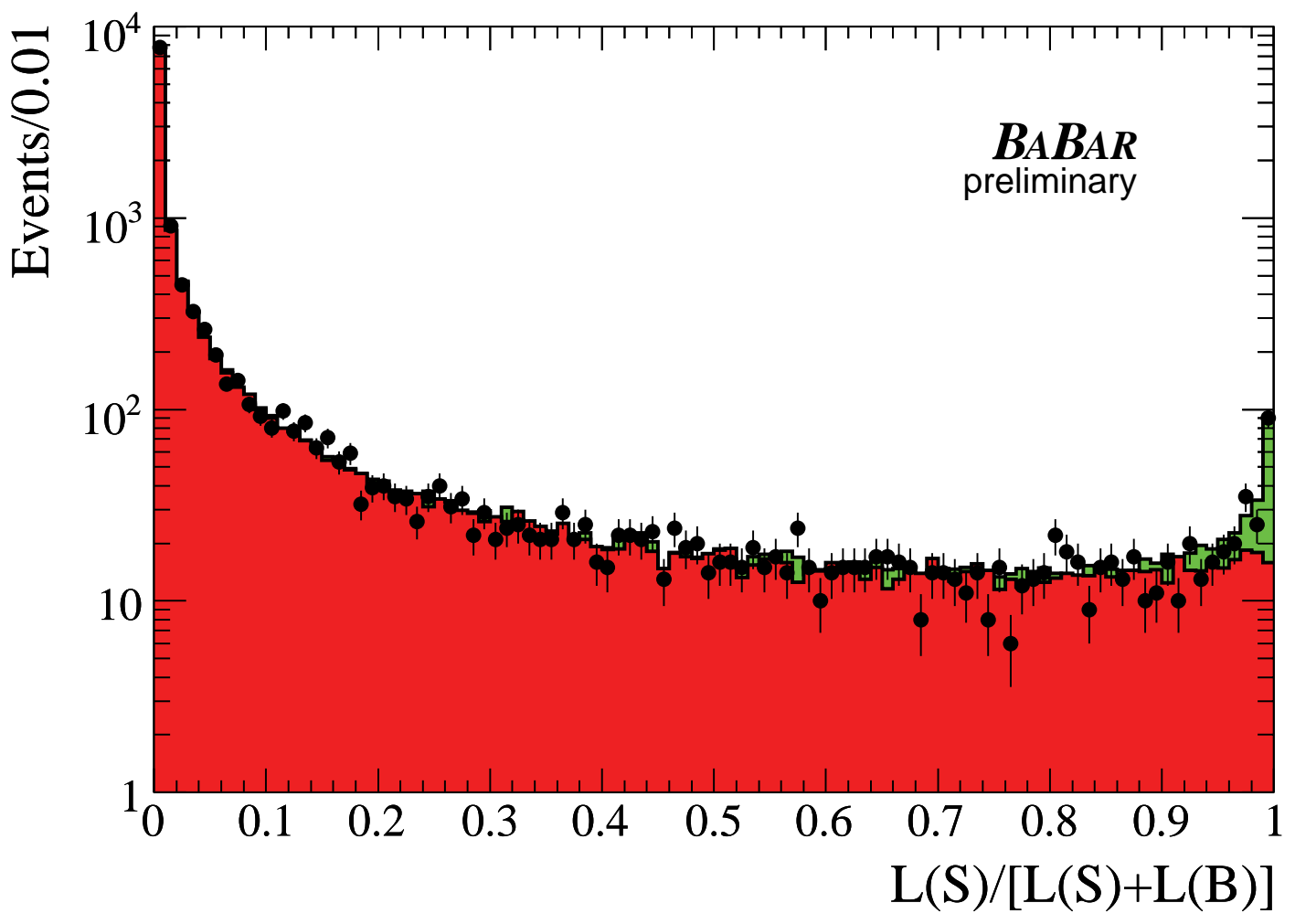

Figure 4.28: The likelihood ratio $\mathcal{L}(s i g) /[\mathcal{L}(s i g)+\mathcal{L}(b k g)]$ for $\omega K_{S}^{0}$. The points represent the on-resonance data, the solid histograms are from pure toy samples of background (red) and background plus signal (green). 
NLLScanPlot_dtSig_S

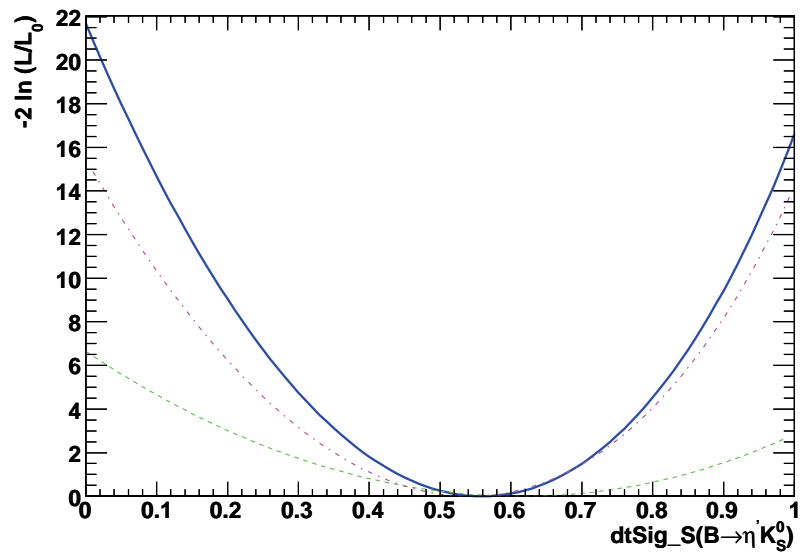

NLLScanPlot_dtSig_C

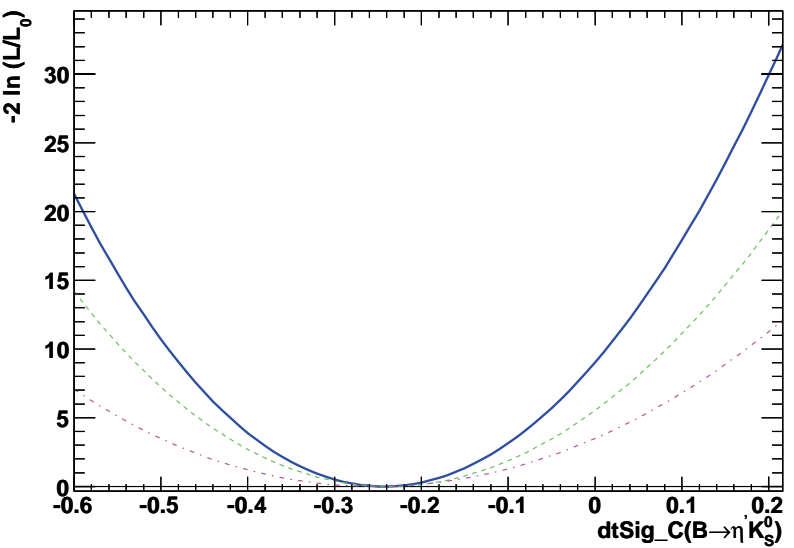

Figure 4.29: $-2 \ln \mathcal{L}$ scan for $S$ (left) and $C$ (right). The solid blue line is for combined neutral sub-decays, the pink dot-dashed line for the $B^{0} \rightarrow \eta_{\eta \pi \pi}^{\prime} K^{0}$ sub-decay and the green dashed line for the $B^{0} \rightarrow \eta_{\rho \gamma}^{\prime} K^{0}$ sub-decay.

Table 4.6: Comparison of combining methods for $214 \mathrm{fb}^{-1}$ (partial results) shown without systematics.

\begin{tabular}{ccc}
\hline \hline & R18 joint fit & R18 NLL curves \\
\hline$S$ & $0.436 \pm 0.143$ & $0.427 \pm 0.139$ \\
\hline$C$ & $-0.281 \pm 0.099$ & $-0.289 \pm 0.097$ \\
\hline \hline
\end{tabular}




\subsection{Systematic Uncertainties for the Branching Fraction Fits}

The main sources of systematic uncertainty in the measurement of branching fractions and charge asymmetries are presented in this section.

\subsubsection{Branching Fraction Systematic Uncertainties}

The systematic errors on the branching fraction measurements may be split into uncertainty on the fit yield and on the efficiency. The former are mostly additive while the latter are multiplicative. The results are summarized in Table 4.7 for the $\eta^{\prime}$ modes and in Table 4.8 for the $\omega$ modes. Each uncertainty is described below.

- ML fit yield: This represents the uncertainties in our fitting procedure. Ideally, we would float the signal PDF parameters in the fit. For modes with a small number of signal events, however, this is not practical. As an alternative, we use control samples to study how well the MC models the data, as discussed in Sec. 3.6. We take the $\Delta E$ resolution scale factor to be $1.05 \pm 0.05, \Delta E$ shift to be $0.0 \pm 2.0 \mathrm{MeV}$ and the $m_{\mathrm{ES}}$ shift to be $0.0 \pm 0.2 \mathrm{MeV}$. For $m_{\omega}$ we take the resolution scale factor to be $1.05 \pm 0.02$ and the shift to be $1.1 \pm 0.1 \mathrm{MeV}$. For $\mathcal{F}$ we vary the mean, asymmetry and RMS of the core Gaussian by the amount determined by the same $B \rightarrow D \pi$ control sample used for $\Delta E$. These variations are then applied, one at a time, to our signal PDFs. The ML fit is rerun taking the quadrature sum of the variations of the fit yield as the systematic error.

- ML fit bias: We assign a systematic uncertainty of one-half the fit bias (see Sec. 3.10.1.2).

- $\underline{B \bar{B}}$ background: For $\eta_{\eta \pi \pi}^{\prime} K^{+}$and $B^{0} \rightarrow \eta_{\eta \pi \pi}^{\prime} K^{0}$ from generic $B \bar{B}$ MC studies we expect backgrounds of 16 and 4 events entering the fits. We estimate a systematic uncertainty of $10 \%$ of this background for these modes. A $B \bar{B}$ 
Table 4.7: Estimates of systematic errors for $\eta^{\prime} K$ branching fraction fits. Multiplicative systematic errors are in percent while additive systematic errors are in events. Each contribution is characterized by whether the contributions for the two $\eta^{\prime}$ submodes are uncorrelated (U) or correlated (C).

\begin{tabular}{lcccc}
\hline \hline Quantity & $\eta_{\eta \pi \pi}^{\prime} K^{+}$ & $\eta_{\rho \gamma}^{\prime} K^{+}$ & $\eta_{\eta \pi \pi}^{\prime} K^{0}$ & $\eta_{\rho \gamma}^{\prime} K^{0}$ \\
\hline Additive errors (events) & & & & \\
Fit yield (U) & 5.5 & 31.7 & 1.4 & 10.2 \\
Fit bias (U) & 0.5 & 15.5 & 1.4 & 17.4 \\
$B \bar{B}$ Bkg (U) & 1.6 & 5.6 & 0.4 & 0.7 \\
\hline Total additive (events) & 5.7 & 35.7 & 2.0 & 20.2 \\
\hline Multiplicative errors (\%) & & & & \\
Track multiplicity (C) & 1.0 & 1.0 & 1.0 & 1.0 \\
Tracking eff/qual (C) & 1.5 & 1.3 & 1.0 & 0.8 \\
$\gamma, \eta$ eff. (C) & 3.0 & 1.8 & 3.0 & 1.8 \\
$K_{S}^{0}$ efficiency (C) & - & - & 1.4 & 1.4 \\
Overlap bug (C) & 0.5 & 0.5 & 0.5 & 0.5 \\
Number $B \bar{B}$ (C) & 1.1 & 1.1 & 1.1 & 1.1 \\
Branching fractions (U) & 3.2 & 3.1 & 3.2 & 3.1 \\
MC statistics (U) & 0.4 & 0.4 & 0.2 & 0.2 \\
cos $\theta_{\mathrm{T}}(\mathrm{C})$ & 0.5 & 0.5 & 0.5 & 0.5 \\
\hline Total multiplicative (\%) & 4.9 & 4.2 & 5.0 & 4.3 \\
\hline Total errors [B $\left.\mathcal{B}\left(10^{-6}\right)\right]$ & & & & \\
Additive & 0.4 & 1.1 & 0.4 & 1.8 \\
Uncorrelated & 2.2 & 2.5 & 2.0 & 2.9 \\
Correlated & 2.5 & 2.0 & 2.3 & 2.1 \\
\hline \hline
\end{tabular}


Table 4.8: Estimates of systematic errors for $\omega$ branching fraction fits. Multiplicative systematic errors are in percent while additive systematic errors are in events.

\begin{tabular}{lccc}
\hline \hline Quantity & $\omega \pi^{+}$ & $\omega K^{+}$ & $\omega K_{S}^{0}$ \\
\hline Additive errors (events) & & & \\
Fit yield & 6.5 & 4.3 & 1.1 \\
Fit bias & 21.9 & 14.7 & 5.2 \\
$B \bar{B}$ Background & 5.8 & 5.5 & 2.3 \\
Primary track PID & 1.8 & 1.5 & - \\
\hline Total additive (events) & 26.2 & 16.3 & 5.8 \\
\hline Multiplicative errors $(\%)$ & & & \\
Track multiplicity & 1.0 & 1.0 & 1.0 \\
Tracking eff/qual & 1.5 & 1.5 & 1.0 \\
$\pi^{0} / \gamma$ eff & 3.0 & 3.0 & 3.0 \\
$K_{S}^{0}$ efficiency & - & - & 1.5 \\
Overlap bug & 0.5 & 0.5 & 0.5 \\
Number $B \bar{B}$ & 1.1 & 1.1 & 1.1 \\
Branching fractions & 0.8 & 0.8 & 0.8 \\
MC statistics & 0.5 & 0.5 & 0.2 \\
cos $\theta_{\mathrm{T}}$ & 0.5 & 0.5 & 0.5 \\
\hline Total multiplicative $(\%)$ & 3.8 & 3.8 & 4.0 \\
\hline Total errors $\left[\mathcal{B}\left(10^{-6}\right)\right]$ & 0.4 & 0.3 & 0.3 \\
\hline \hline
\end{tabular}


background component is included in the fit for the other modes. In these cases, we vary the branching fraction for the top 4-8 contributing $B \bar{B}$ modes individually by the uncertainty as shown in Tables 3.3-3.5. The changes in the signal yield are taken in quadrature and scaled to account for the unvaried modes to determine the systematic uncertainty. For $\omega K_{S}^{0}$ where the $B \bar{B}$ yield is fixed, we fixed the $B \bar{B}$ yield to 0 or 92 (double the nominal value) and take the average change in the signal yield as a systematic uncertainty.

- Particle ID: The PID efficiency is determined from PID tables where data control samples determine efficiencies with an uncertainty of $\sim 0.1 \%$ when integrated over our samples. Thus we take the systematic error to be negligible. For the $B^{+} \rightarrow \omega h^{+}$analysis, we include the uncertainties of the PID parameterization for the prompt charged track by varying the PDF in our fit by an amount determined from data control samples described in 3.6.7. Our fit uses the shape from this control sample for charged pions. We take the systematic error to be the change in the signal yield when the shape for kaons is used.

- Track multiplicity: This is for the cut on the minimum number of tracks in the event. We require the reconstruction of at least one track from the other $B$ decay. The signal MC inefficiency for this cut is at most $1-2 \%$. The systematic error is designed to cover any difference between data and MC in the $B$ multiplicity spectrum.

- Track finding/efficiency: The study of absolute tracking efficiency provides a systematic error associated with the correction tables for GoodTracksLoose tracks of $0.5 \%$ per track and $0.4 \%$ for GoodTracksVeryLoose based on tau-pair event studies.

- $\pi^{0}, \eta$ and $\gamma$ finding: The Neutral Identification and Reconstruction working 
group in BABAR has determined that the photon efficiency is overestimated in MC. We follow the $\pi^{0}$ efficiency correction procedures and apply a systematic uncertainty of $3 \%$ per $\pi^{0}[49]$ based on studies of tau-pair events with decays to $\pi^{0}$ 's. For $\eta \rightarrow \gamma \gamma$ we also estimate an uncertainty of $3 \%$. For the single photon in $\eta^{\prime} \rightarrow \rho \gamma$ decays we estimate an uncertainty of $1.8 \%$.

- $K_{S}^{0}$ efficiency: We have determined the $K_{S}^{0}$ efficiency correction and associated systematic errors following the recipe described in [50] for our MC based on studies of inclusive $K_{S}^{0}$ channels in data and MC.

- Overlaps function bug: We estimate a systematic error of $0.5 \%$ for a code reconstruction bug described in App. G.

- Number $B \bar{B}$ : The uncertainty of the absolute $B \bar{B}$ pair counting calculation is estimated to be $1.1 \%$.

- Branching fractions of daughters: This is taken as the uncertainty of the daughter branching fractions from the PDG [2].

- MC statistics: This is calculated for the number of MC signal events simulated for each decay.

- Event shape: There are two variables used for event shape, $\cos \theta_{\mathrm{T}}$ and $\mathcal{F}$. For $\cos \theta_{\mathrm{T}}$ the expectation is that the distribution of signal $\mathrm{MC}$ for this variable should be nearly flat. We take the systematic uncertainty to be one-half of the difference between the observed signal MC efficiency of the $\cos \theta_{\mathrm{T}}$ cut used for each analysis and the expectation of a flat distribution. For $\mathcal{F}$, the systematic uncertainty is included in the ML fit yield discussed above.

- Trigger efficiency: The $B$ counting group measured the trigger efficiency for multi-hadron events to be in excess of $99.93 \%$. We neglect the systematic error on the tiny inefficiency from this source. 
In rare $B$ decays the statistical errors have traditionally dominated the overall measurement uncertainties. This is no longer true for the large datasets currently available in channels with relatively large signal yields, such as the $\eta^{\prime} K$ modes. The $\omega$ modes are still limited by statistics, but the $\omega h^{+}$channels are also approaching the transition into a systematics limited measurement.

The largest systematic uncertainties are from the daughter branching fractions taken from the PDG. The best of these measurements do not come from BABAR and would be difficult to improve. The next largest source of systematic uncertainty comes from uncertainty in neutrals reconstruction efficiency for photons and $\eta$ mesons. This source of uncertainty could be improved with a better understanding of the detector response.

\subsubsection{Charge Asymmetry Systematic Uncertainties}

Most of the systematic uncertainties found for the branching fraction measurements cancel for the $\mathcal{A}_{c h}$ because the charge conjugate decays are so similar. The primary sources of bias are: tracking differences between oppositely charged tracks; PID differences; differences due to the interaction cross sections in the detector material; the effect of $B \bar{B}$ background; and the fit bias dilution.

We have studied such bias effects in a number of ways including control samples and fits to the data. For MC, we find a charge asymmetry of $-0.8 \pm 0.2 \%$ for $\eta_{\eta \pi \pi}^{\prime} K^{+}$ and $\eta_{\rho \gamma}^{\prime} K^{+}$and $-0.6 \pm 0.4 \%(-0.7 \pm 0.4 \%)$ for $\omega K^{+}\left(\omega \pi^{+}\right)$. For $q \bar{q}$ data we find $-3.3 \pm$ $2.5 \%(-1.7 \pm 0.4 \%)$ for $\eta_{\eta \pi \pi}^{\prime} K^{+}\left(\eta_{\rho \gamma}^{\prime} K^{+}\right)$and $-0.9 \pm 0.7 \%(-1.0 \pm 0.5 \%)$ for $\omega K^{+}$ $\left(\omega \pi^{+}\right)$. In addition, we have calculated the $\mathcal{A}_{c h}$ values for the $\eta^{\prime}(\rho \gamma) \pi^{+}$channel and find $-0.2 \pm 0.4 \%$ for $\mathrm{MC}$ and $0.1 \pm 0.4 \%$ for $q \bar{q}$.

We have evidence that the $q \bar{q} \mathcal{A}_{c h}$ is not constant as a function of polar angle, though the overall effect averages to zero within uncertainties. It is not clear why we see a possible difference between the values in data and MC. 
Ultimately, we use a combination of the studies mentioned above and calculations of detector interaction effects to conclude that an approximate average $\mathcal{A}_{c h}$ bias is $-0.010 \pm 0.005$ for modes with a primary kaon and $0.000 \pm 0.005$ for modes with a primary Pion. We correct for the bias and include the uncertainty as a systematic error.

To study the potential effect of bias on the $\mathcal{A}_{c h}$ from $B \bar{B}$ background, we fixed the $B \bar{B} \mathcal{A}_{c h}$ to $\pm 10 \%$ in the fit and take the change in the signal $\mathcal{A}_{c h}$ as a systematic uncertainty. We find a systematic uncertainty of 0.006 for $\eta_{\rho \gamma}^{\prime} K^{+}, 0.003$ for $\omega \pi^{+}$, and 0.002 for $\omega K^{+}$. The total systematic errors with appropriate corrections to the central values are shown in Tables 4.1 and 4.2.

The fit bias results in a dilution of the measured central value and error in the $\mathcal{A}_{c h}$. We estimate a systematic uncertainty of $100 \%$ of the multiplicative correction applied to account for this bias in $\omega h^{+}$.

\subsection{Systematic Uncertainties for Time-dependent Asymmetry Fits}

Contributions to the systematic uncertainties in $S$ and $C$ are summarized in Table 4.9 and described here:

- For PDF shapes, we estimate the errors principally by variation of the fit parameters. In Table 4.10, we summarize all of the variations and their results. For the $\eta^{\prime} K_{S}^{0}$ results, we calculate the systematic error using the joint submode fit. All changes are combined in quadrature to obtain an error of 0.010 for $S$ and 0.016 for $C$ for $\eta^{\prime} K_{S}^{0}$ and an error of 0.008 for $S$ and 0.020 for $C$ for $\omega K_{S}^{0}$.

- Toy studies (Sec. 4.2.2) show that there may be a bias in $S$ due to $B \bar{B}$ background. We correct for the dilution of $S$ in $\eta_{\rho \gamma}^{\prime} K_{S}^{0}$ due to $B \bar{B}$ background and estimate an uncertainty of 0.01 in $S$ in both the $\eta^{\prime}$ and $\omega$ result due to this effect. 
Due to uncertainty of the $C P$ content in the $B \bar{B}$ background we perform fits floating $\mathrm{S}$ and $\mathrm{C}$ in the $B \bar{B}$ background for the $\eta^{\prime} K_{S}^{0}$ channels. We find $\mathrm{S}$ and $\mathrm{C}$ in $B \bar{B}$ background consistent with zero and find a change of $0.005(0.004)$ in S (C) for our signal, which we take as an additional systematic error for a total of 0.011 for $\mathrm{S}$ and 0.004 for $\mathrm{C}$. For $\omega K_{S}^{0}$ the $B \bar{B}$ contribution is much smaller and we cannot float the $C P$ parameters. With reasonable assumptions about the $C P$ content of the $B \bar{B}$ background, we find a negligible change in $S$ and $C$ so no additional systematic error is required.

- Toy studies (Sec. 3.10.3.2) show that there are no significant biases in $S$ and $C$ for the $\eta^{\prime} K_{S}^{0}$ modes other than the $B \bar{B}$ background issue just discussed. We assign an uncertainty of 0.01 for the modeling of the signal to cover the statistical uncertainties of these toy studies. For $\omega K_{S}^{0}$ we find a correction of -0.015 is needed for $S$ and that no correction is needed for $C$ and apply a systematic error of 0.02 for $S$ and 0.01 for $C$ to cover the uncertainties in these estimates.

- We vary the SVT alignment parameters in the signal Monte Carlo events by the amount of mis-alignment found in the real data, and assign the resulting shift in the fit results as a systematic error. There are five different SVT configurations which are considered. Four configurations simulate time dependent mis-alignment and one simulates the radius-dependent $z$ shift of entire layers. The difference for each configuration between the nominal values of $S$ and $C$ and those from the mis-aligned configuration are taken in quadrature to determine the systematic error, which is found to be quite small in all channels for both $S$ and $C$.

- We vary the beam-spot $y$ position and error in the signal Monte Carlo events by a reasonable expectation of its uncertainty. The change in $S$ and $C$ from the nominal values are taken as a systematic uncertainty, which is also found to be 
quite small.

- The effect of interference between the CKM-suppressed $b \rightarrow u \bar{c} d$ amplitude with the favored $b \rightarrow c \bar{u} d$ amplitude for some tag-side $B$ decays [53]. We use our best fit values of $S$ and $C$ as inputs for toy studies estimating the effect of this interference. We find an uncertainty of 0.002 for $\mathrm{S}$ and 0.014 for $\mathrm{C}$ for all channels at the $68 \%$ confidence level.

- We include a systematic uncertainty for the appropriateness of using $\Delta t$ parameters from BReco data for the signals. The resolution on the signal side is not completely negligible so the resolution function could be slightly different for the different channels. We remove this effect to first order by using $\Delta t / \sigma_{\Delta t}$ instead of $\Delta t$ as the fit variable. We evaluate the size of this any remaining effect by finding the difference between toy results with BReco MC signal $\Delta t$ parameters and those for signal MC as shown in Table 3.11. We estimate a systematic error of 0.004 for $S$ and 0.012 for $C$.

Summing all systematic errors in quadrature, we find 0.02 for $S$ and 0.03 for $C$ for all channels.

Table 4.9: Estimates of systematic errors for $\Delta t$ fits.

\begin{tabular}{lcccc}
\hline \hline Source of error & \multicolumn{2}{c}{$\eta^{\prime} K_{S}^{0}$} & \multicolumn{2}{c}{$\omega K_{S}^{0}$} \\
& $\sigma(S)$ & $\sigma(C)$ & $\sigma(S)$ & $\sigma(C)$ \\
\hline PDF Shapes & 0.010 & 0.016 & 0.008 & 0.020 \\
$B \bar{B}$ Background & 0.011 & 0.004 & 0.010 & 0.000 \\
$\Delta t$ modeling & 0.010 & 0.010 & 0.020 & 0.010 \\
SVT alignment & 0.001 & 0.001 & 0.000 & 0.002 \\
Beam position/size & 0.001 & 0.001 & 0.001 & 0.001 \\
Tag-side interference & 0.002 & 0.014 & 0.002 & 0.014 \\
BReco signal shape & 0.004 & 0.012 & 0.004 & 0.012 \\
\hline Total & 0.019 & 0.027 & 0.024 & 0.029 \\
\hline \hline
\end{tabular}


Table 4.10: Results of systematic variations for $\Delta t$ fits. We show the nominal values, the amount that we vary these, the source of this variation amount, and the change of $S$ and $C$ for this amount of variation. We group similar quantities together after combining their variations in quadrature.

\begin{tabular}{lcccccc}
\hline \hline Quantity & Nominal \pm variation & Source of & \multicolumn{2}{c}{$\eta^{\prime} K_{S}^{0}$} & \multicolumn{2}{c}{$\omega K_{S}^{0}$} \\
& & variation & $\delta S$ & $\delta C$ & $\delta S$ & $\delta C$ \\
\hline$\Delta m_{d}$ & $0.507 \pm 0.005$ & PDG & 0.0040 & 0.0023 & 0.0016 & 0.0061 \\
$\tau_{B}$ & $1.530 \pm 0.009$ & PDG & 0.0005 & 0.0006 & 0.0006 & 0.0001 \\
$w$ & Table B.1 & Table B.1 & 0.0040 & 0.0113 & 0.0060 & 0.0113 \\
$\Delta w$ & Table B.1 & Table B.1 & 0.0008 & 0.0108 & 0.0030 & 0.0099 \\
Signal $f_{\text {cat }}$ & Table B.1 & Table B.1 & 0.0009 & 0.0004 & 0.0004 & 0.0005 \\
Signal $\Delta t$ & Table B.2 & Table B.2 & 0.0057 & 0.0032 & 0.0027 & 0.0030 \\
$\Delta E$ scale & $1.05 \pm 0.05$ & Table 3.2 & 0.0054 & 0.0012 & 0.0023 & 0.0060 \\
$m_{\text {ES shift }}$ & Table 3.2 & Table 3.2 & 0.0019 & 0.0012 & 0.0021 & 0.0063 \\
$\mathcal{F}$ & PDF fit \pm error & Floating fit & 0.0001 & 0.0002 & 0.0019 & 0.0054 \\
$m_{\omega}$ scale & Table 3.2 & Table 3.2 & - & - & 0.0016 & 0.0026 \\
$m_{\omega}$ shift & Table 3.2 & Table 3.2 & - & - & 0.0002 & 0.0005 \\
\hline Total & & & 0.0099 & 0.0162 & 0.0084 & 0.0196 \\
\hline \hline
\end{tabular}




\section{Chapter 5}

\section{Discussion}

This thesis presents measurements of the branching fractions for the decays $B^{0} \rightarrow$ $\eta^{\prime} K_{S}^{0}, B^{+} \rightarrow \eta^{\prime} K^{+}, B^{+} \rightarrow \omega h^{+}$, and $B^{0} \rightarrow \omega K_{S}^{0}$. For the charged modes, the charge asymmetry is measured, and for the neutral modes the time-dependent $C P$ asymmetry is measured. The results are summarized in Table 5.1.

Table 5.1: Branching fraction, charge asymmetry and $C P$ asymmetry final results for $B \rightarrow \eta^{\prime} K, B^{+} \rightarrow \omega \pi^{+}$, and $B \rightarrow \omega K$ final states. The first uncertainty is statistical and the second systematic.

\begin{tabular}{lcccc}
\hline \hline & $\mathcal{B}\left(\times 10^{-6}\right)$ & $\mathcal{A}_{c h}(\%)$ & $\mathrm{S}$ & $\mathrm{C}$ \\
$\eta^{\prime} K^{+}$ & $70.0 \pm 1.5 \pm 2.8$ & $1.0 \pm 2.2 \pm 0.6$ & - & - \\
$\eta_{\eta \pi \pi}^{\prime} K^{+}$ & $68.2 \pm 2.3 \pm 3.3$ & $-0.5 \pm 3.3 \pm 0.5$ & - & - \\
$\eta_{\rho \gamma}^{\prime} K^{+}$ & $72.2 \pm 2.1 \pm 3.2$ & $2.2 \pm 2.8 \pm 0.8$ & - & - \\
\hline$\eta^{\prime} K^{0}$ & $66.6 \pm 2.6 \pm 2.8$ & - & $0.56 \pm 0.12 \pm 0.02$ & $-0.24 \pm 0.08 \pm 0.03$ \\
$\eta_{\eta \pi \pi}^{\prime} K^{0}$ & $60.7 \pm 3.7 \pm 3.0$ & - & $0.61 \pm 0.23 \pm 0.02$ & $-0.25 \pm 0.13 \pm 0.03$ \\
$\eta_{\rho \gamma}^{\prime} K^{0}$ & $72.8 \pm 3.5 \pm 3.7$ & - & $0.53 \pm 0.13 \pm 0.02$ & $-0.24 \pm 0.10 \pm 0.03$ \\
\hline$\omega \pi^{+}$ & $6.7 \pm 0.5 \pm 0.4$ & $-2 \pm 8 \pm 1$ & - & - \\
$\omega K^{+}$ & $6.3 \pm 0.5 \pm 0.3$ & $-1 \pm 7 \pm 1$ & - & - \\
$\omega K_{S}^{0}$ & $5.6 \pm 0.8 \pm 0.3$ & - & $0.62_{-0.29}^{+0.25} \pm 0.02$ & $-0.39_{-0.24}^{+0.25} \pm 0.03$ \\
\hline \hline
\end{tabular}

The branching fractions based on the 2000-2006 BABAR dataset with $347 \mathrm{fb}^{-1}$ of data containing $383 \mathrm{M} B \bar{B}$ pairs represent improvements in the uncertainties of $\sim 15-$ $30 \%$ over the previous BABAR results, which are the most precise published results. All the improved results are consistent with the theoretical expectations. The explanation of enhanced branching fractions due to constructive interference between the leading-order 
penguin diagrams in $\eta^{\prime} K$ remains valid and the precision of the measurements increases confidence in this conclusion. In the $\omega$ decays, the uncertainties, both experimental and theoretical, are still sufficiently large that no definite conclusions can be drawn at this point.

The charge asymmetry results are all fully consistent with zero as expected. These new branching fraction and charge asymmetry results are undergoing collaboration review and a manuscript is in preparation to be submitted to Physical Review D.

The time-dependent results for $B^{0} \rightarrow \eta^{\prime} K_{S}^{0}$ have been combined with similar results from BABAR from several other subdecay modes to the same final state: $B^{0} \rightarrow$ $\eta^{\prime}\left(\eta\left(\pi^{+} \pi^{-} \pi^{0}\right) \pi^{+} \pi^{-}\right) K_{S}^{0}\left(\pi^{+} \pi^{-}\right), B^{0} \rightarrow \eta^{\prime}\left(\eta(\gamma \gamma) \pi^{+} \pi^{-}\right) K_{S}^{0}\left(\pi^{0} \pi^{0}\right), B^{0} \rightarrow \eta^{\prime}(\rho \gamma) K_{S}^{0}\left(\pi^{0} \pi^{0}\right)$, and $B^{0} \rightarrow \eta^{\prime}\left(\eta(\gamma \gamma) \pi^{+} \pi^{-}\right) K_{L}^{0}$. These results are combined to give the final published result [54],

$$
S_{\eta^{\prime} K^{0}}=0.58 \pm 0.10 \pm 0.03, \quad C_{\eta^{\prime} K^{0}}=-0.16 \pm 0.07 \pm 0.03
$$

Including systematics, the statistical significance of the measurement of S is 5.5 standard deviations from zero. This represents the first-observation of $C P$ violation in $b \rightarrow s$ penguin-dominated $B$ meson decays.

The time-dependent result for $B^{0} \rightarrow \omega K_{S}^{0}$ was prepared as a preliminary result and presented at ICHEP 2006 on a slightly smaller dataset than presented here $(347 \mathrm{M}$ $B \bar{B}$ pairs) [55], with the results

$$
S_{\omega K_{S}^{0}}=0.62_{-0.30}^{+0.25} \pm 0.02, \quad C_{\omega K_{S}^{0}}=-0.43_{-0.23}^{+0.25} \pm 0.03
$$

The preliminary result is an update to the original BABAR measurement [56] of the time-dependent $C P$ asymmetries in $\omega K_{S}^{0}$ that we submitted in 2006.

The same quantities have been measured by Belle with the results shown in Table 1.2. Generally, the branching fractions agree well between the two experiments. Belle finds slightly lower branching fractions for $B^{0} \rightarrow \eta^{\prime} K_{S}^{0}$ and $B^{0} \rightarrow \omega K_{S}^{0}$ and a 
slightly larger branching fraction for $\omega K^{+}$, though the results are consistent within uncertainties. Belle also observes $S \neq 0$ with $5 \sigma$ significance and no evidence is found for direct $C P$ violation, in good agreement with BABAR.

The great interest in the time-dependent $C P$ asymmetry results for $b \rightarrow s$ penguin-dominated decays such as $\eta^{\prime} K_{S}^{0}$ and $\omega K_{S}^{0}$ stems from the potential to observe new physics effects in the loop. In Fig. 5.1 we show a compilation of the current experimental values for $\sin 2 \beta_{\text {eff }}$ from measurements of $\mathrm{S}$ accounting for $\eta= \pm 1$ for all measurements from $b \rightarrow s$ penguin decays [16].

The first-order SM expectation is that these $b \rightarrow s$ penguin channels have the same $C P$-violating effects as $c \bar{c} K^{0}$ final states, i.e. $\sin 2 \beta_{\text {eff }}=\sin 2 \beta$. As shown in Fig. 5.1 the experimental results show a trend toward values of $S$ below this SM expectation. Even more encouraging as a sign of a potential deviation from the SM expectation is that the theoretically predicted values for the SM $\Delta S=\sin 2 \beta_{\text {eff }}-\sin 2 \beta$, due to contributions from non-leading-order diagrams, tend to be positive [22, 57, 58].

One way to quantify the results is to compute a weighted average of all the $b \rightarrow s$ penguin results. The current value of this average for $\sin 2 \beta_{\text {eff }}$ is $0.53 \pm 0.05$ compared with $\sin 2 \beta$ from $c \bar{c} K_{S}^{0}$ at $0.68 \pm 0.03$, where the uncertainties include both statistical and systematic effects [16]. This represents a $2.6 \sigma$ discrepancy. This average gives some indication of the overall trend, but must be considered with caution because each channel must have the SM $\Delta S$ effects evaluated individually and in general need not be affected by new physics contributions in the same way.

No individual channel currently shows more than $\sim 2 \sigma$ deviation. The most precise of this series of measurements is $\eta^{\prime} K^{0}$, where we now observe a $1 \sigma$ deviation from $\sin 2 \beta$. The measurements for the time-dependent $C P$ asymmetry parameters are still statistics limited, and are projected to be so through the end of the $B$ factory experiments. A projected doubling of the data sets will allow for a reduction in the errors by $\sim 30 \%$. Continued improvements in theoretical techniques and more precise 


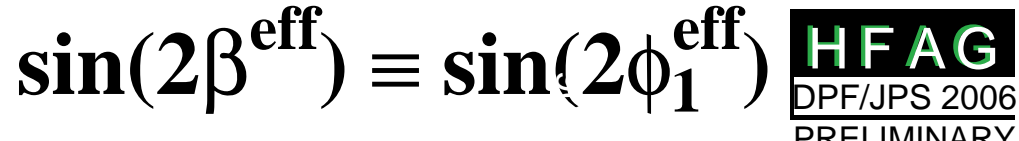

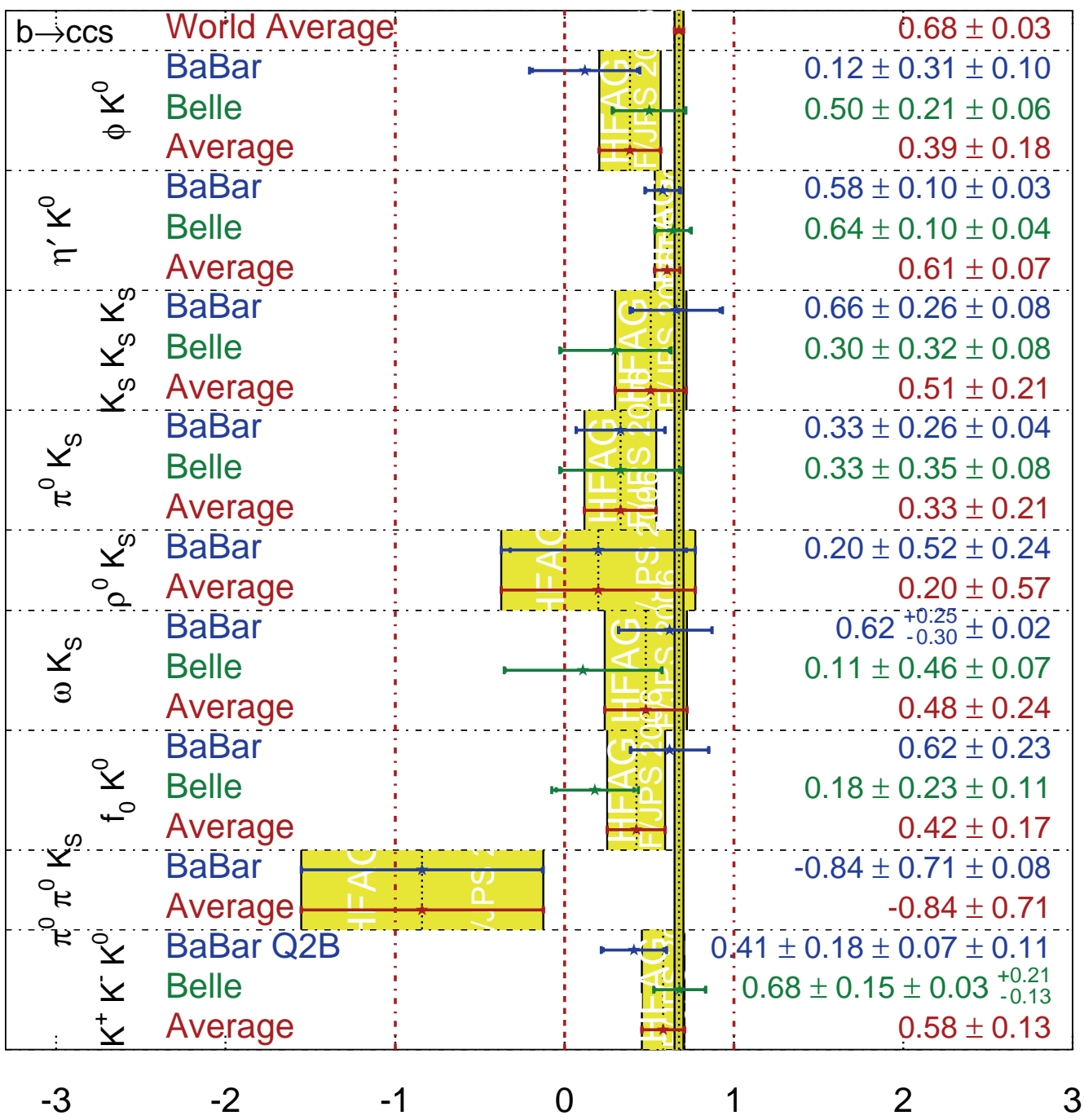

Figure 5.1: Experimental results for $\sin 2 \beta_{\text {effective }}=-\eta_{f_{C P}} S_{f_{C P}}$ for all measured decay channels. The narrow gold band shows the value of $\sin 2 \beta$ as measured in $c \bar{c} K_{S}^{0}$ states. 
measurements of $\mathrm{SU}(3)$ related modes also would allow for better determinations of $\Delta S$ in the SM and therefore potential new physics effects.

The measured experimental values for $\mathrm{C}$, the direct $C P$ violation parameter, are shown in Fig. 5.2. No clear trend is evident across the various decay channels in this case and the world average measurements of all modes are within $1.5 \sigma$ of zero. 


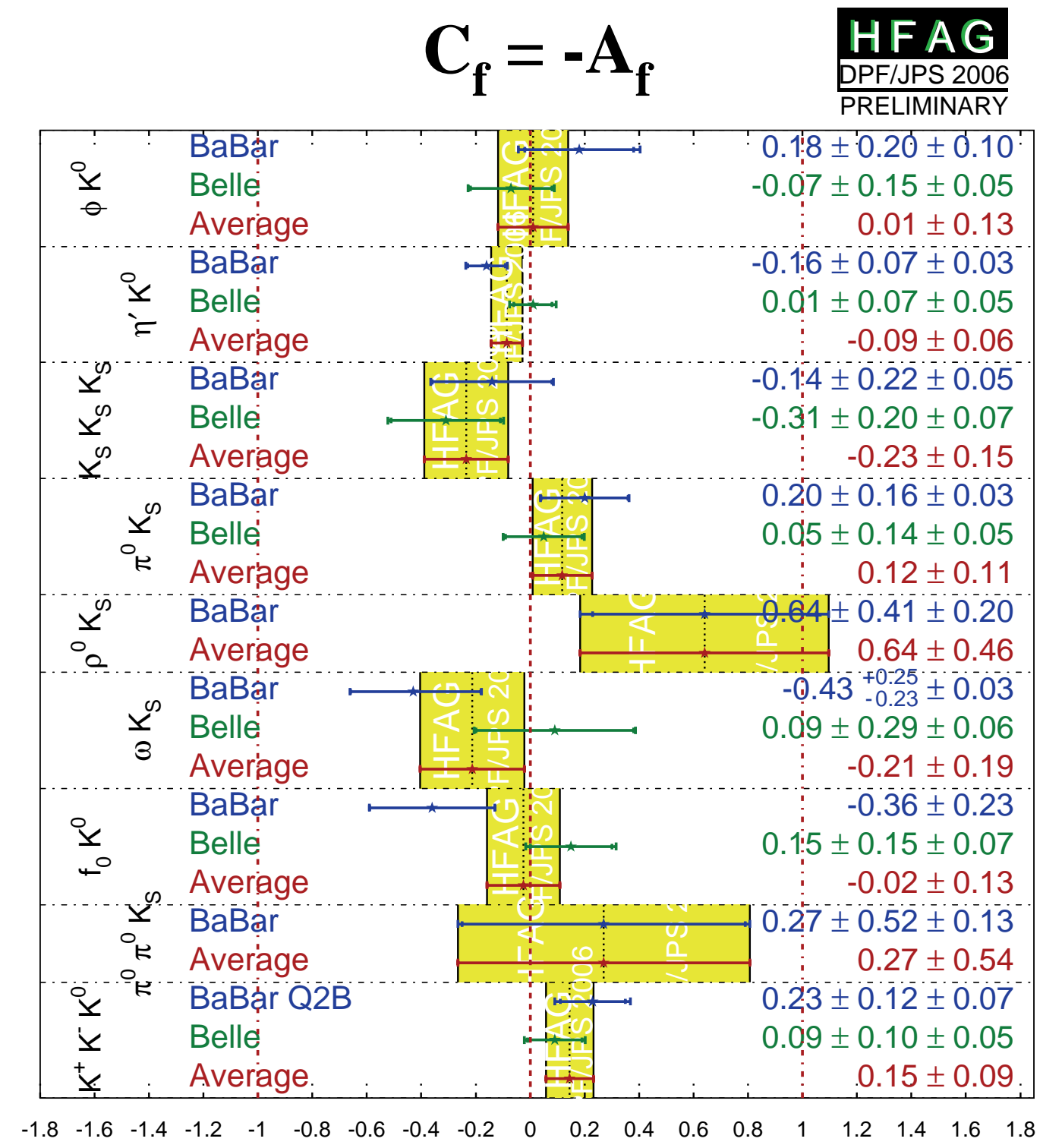

Figure 5.2: Experimental results for $C$ for all measured decay channels. 


\section{Appendix A}

\section{Particle Lists}

Here specific requirements are given for particle lists used in this analysis.

\section{A.1 GoodTracksVeryLoose}

- Maximum momentum $10 \mathrm{GeV}$

- Distance of closest approach to the beam spot in the $x-y$ plane within $1.5 \mathrm{~cm}$

- Distance of closest approach in $z$ within $10 \mathrm{~cm}$

\section{A.2 GoodTracksLoose}

- All GoodTracksVeryLoose requirements

- Minimum transverse momentum $100 \mathrm{MeV}$

- Minimum 12 drift chamber hits

\section{A.3 ChargedTracks}

- All non-zero charge candidates 


\section{Appendix B}

\section{Fits to BReco Data}

The results from the fits to BReco data are shown here.

Table B.1: BReco signal tagging fractions $(f)$, mistag fractions $(\langle w\rangle)$, mistag differences $(\Delta w)$, and tag efficiency differences $(\mu)$ for each tagging category determined from fit to the neutral BReco sample.

\begin{tabular}{lcccc}
\hline \hline Category & $f_{\text {sig }}$ & $\langle w\rangle$ & $\Delta w$ & $\mu$ \\
\hline Lepton & $0.0867 \pm 0.0008$ & $0.0297 \pm 0.0033$ & $-0.0015 \pm 0.0064$ & $0.0056 \pm 0.0113$ \\
KaonI & $0.1096 \pm 0.0009$ & $0.0535 \pm 0.0038$ & $-0.0057 \pm 0.0071$ & $0.0025 \pm 0.0110$ \\
KaonII & $0.1721 \pm 0.0010$ & $0.1546 \pm 0.0039$ & $-0.0044 \pm 0.0066$ & $0.0027 \pm 0.0096$ \\
KorPI & $0.1377 \pm 0.0010$ & $0.2349 \pm 0.0048$ & $-0.0237 \pm 0.0078$ & $-0.0167 \pm 0.0107$ \\
Pions & $0.1438 \pm 0.0010$ & $0.3295 \pm 0.0051$ & $0.0524 \pm 0.0078$ & $-0.0284 \pm 0.0107$ \\
Other & $0.0961 \pm 0.0008$ & $0.4193 \pm 0.0063$ & $0.0459 \pm 0.0094$ & $0.0245 \pm 0.0124$ \\
Untagged & $0.2540 \pm 0.0012$ & 0.50 & 0 & 0 \\
\hline \hline
\end{tabular}

Table B.2: Summary of BReco signal resolution function parameters.

\begin{tabular}{lc}
\hline \hline Parameter & $B^{0}$ \\
\hline Scale Lepton (core) & $1.0631 \pm 0.0489$ \\
Scale Not Lepton (core) & $1.0985 \pm 0.0235$ \\
$\delta(\Delta t)$ Lepton (core) & $-0.0709 \pm 0.0321$ \\
$\delta(\Delta t)$ No Lepton (core) & $-0.1805 \pm 0.0145$ \\
$f($ core) & $0.8888 \pm 0.0092$ \\
Scale (tail) & 3.0 (fixed) \\
$\delta(\Delta t)$ (tail) & $-1.1140 \pm 0.1380$ \\
$f($ outlier $)$ & $0.0033 \pm 0.0006$ \\
Scale (outlier) & 8.0 (fixed) \\
$\delta(\Delta t)$ (outlier) $(\mathrm{ps})$ & 0.0 (fixed) \\
\hline \hline
\end{tabular}




\section{Appendix $\mathrm{C}$}

\section{PDF Libraries}

We show here for each decay mode the signal and background PDFs used in ML fits. We show also the linear correlation coefficients between the input variables used in the ML fits as well as the final values of the fits. A description of the variable names used in the fit is given in Table C.1.

Signal PDFs are determined from MC signal events. For continuum background PDFs we have used on-peak sidebands. For $B \bar{B}$ background PDFs we have used MC events. 
Table C.1: Explanation of variable names used in RooRarFit.

\begin{tabular}{lc}
\hline \hline Q2BFit name & Description \\
\hline Frac nBkg chgCat Plus & fraction of $B^{+}$events in $q \bar{q}$ \\
Frac nChls chgCat Plus & fraction of $B^{+}$events in $B \bar{B}$ \\
Frac nSig chgCat Plus & fraction of $B^{+}$events in signal \\
Frac nBkg tagCat 04Tx & fraction of $q \bar{q}$ yield in tagging category x \\
deBkg P01 & $\Delta E$ background slope \\
dtBkg fracC & $\Delta t$ fraction of core Gaussian for $q \bar{q}$ \\
dtBkg fracO0 & $\Delta t$ fraction of outlier Gaussian for $q \bar{q}$ \\
dtBkg meanC & $\Delta t$ core Gaussian mean for $q \bar{q}$ \\
dtBkg meanT & $\Delta t$ tail Gaussian mean for $q \bar{q}$ \\
dtBkg sigmaC & $\Delta t$ core Gaussian $\sigma$ for $q \bar{q}$ \\
dtBkg sigmaT & $\Delta t$ tail Gaussian $\sigma$ for $q \bar{q}$ \\
dtSig C & $C P$ parameter $C$ \\
dtSig S & $C P$ parameter $S$ \\
fisBkgC asym & $\mathcal{F}$ core $($ bifurcated) Gaussian asymmetry for $q \bar{q}$ \\
fisBkgC mean & $\mathcal{F}$ core Gaussian mean for $q \bar{q}$ \\
fisBkgC rms & $\mathcal{F}$ core Gaussian average width for $q \bar{q}$ \\
mesBkg c & $m_{\text {ES }}$ Argus exponent for $q \bar{q}$ \\
mObkg fracS & $\omega$ mass true $\omega$ fraction in $q \bar{q}$ \\
mOPolyBkg P01 & $\omega$ mass slope in $q \bar{q}$ \\
nBkg & $q \bar{q}$ signal yield \\
nChls & $B \bar{B}$ signal yield \\
nSig & Signal yield \\
\hline \hline
\end{tabular}




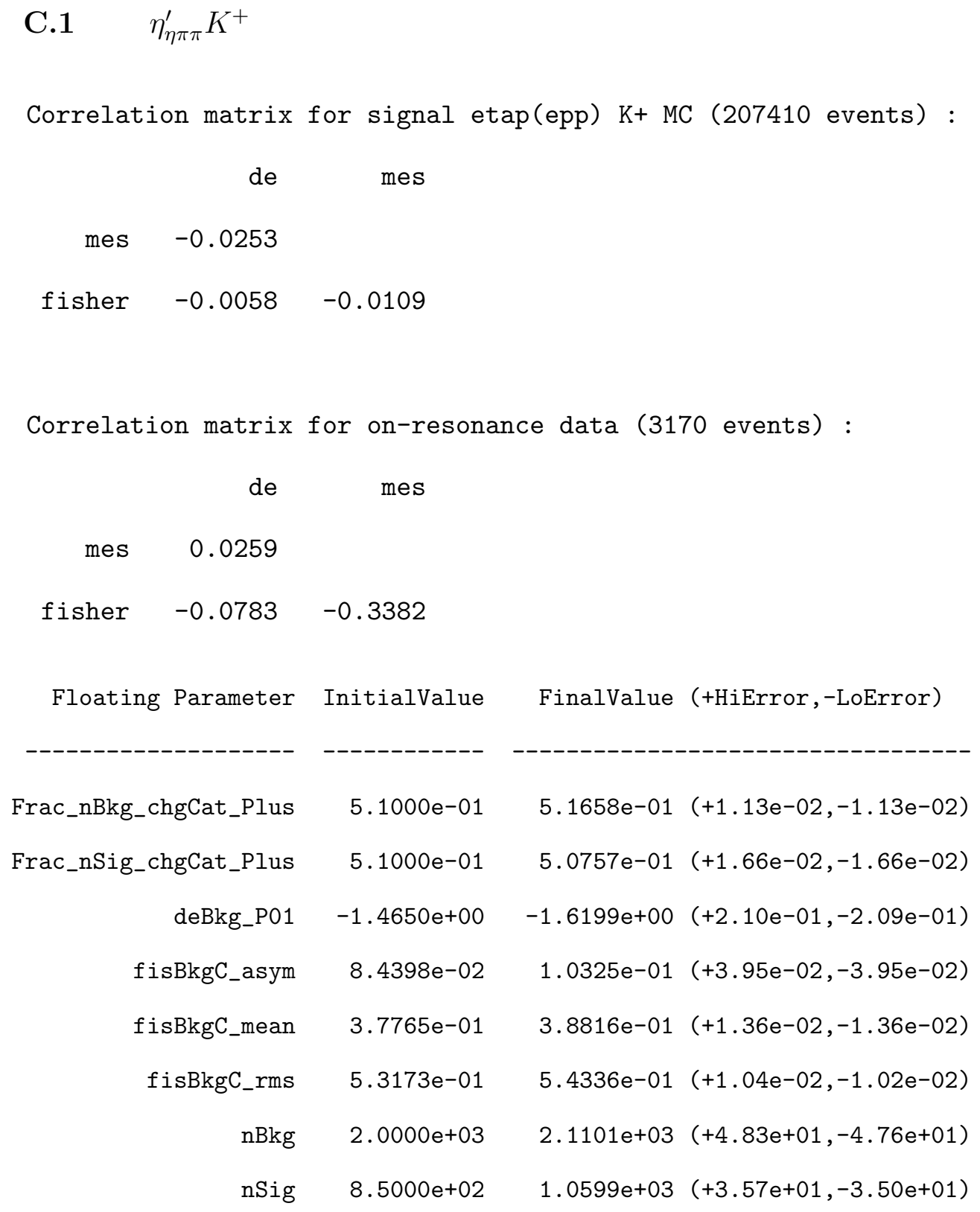



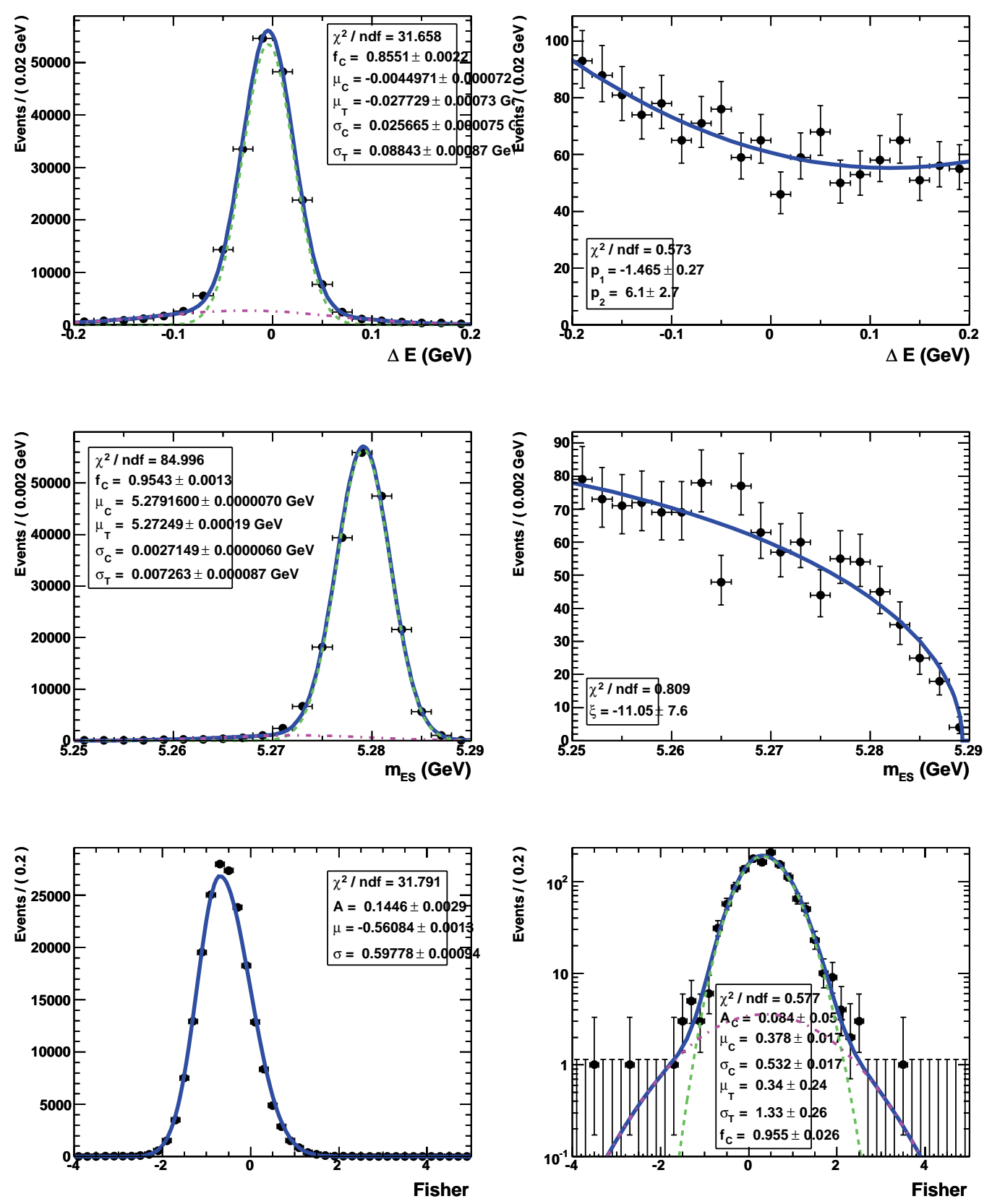

Figure C.1: PDFs for $\eta_{\eta \pi \pi}^{\prime} K^{+}$(from top to bottom) $\Delta E, m_{\mathrm{ES}}$, and $\mathcal{F}$. Signal MC (left) and on-peak sidebands (right). 


\section{C.2 $\eta_{\rho \gamma}^{\prime} K^{+}$}

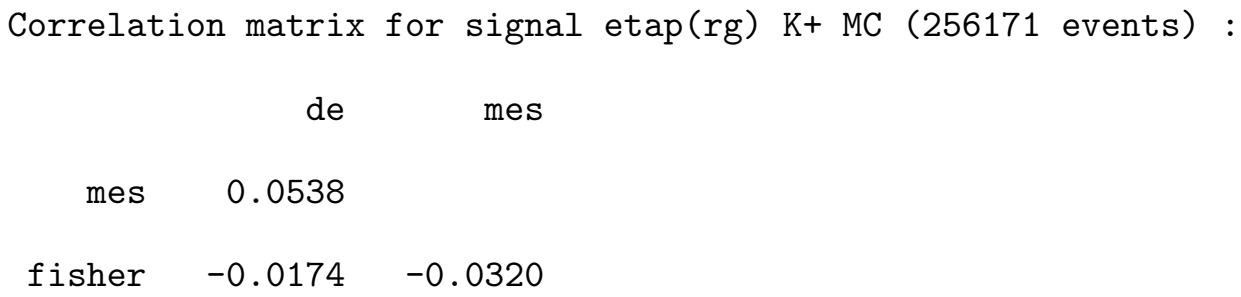



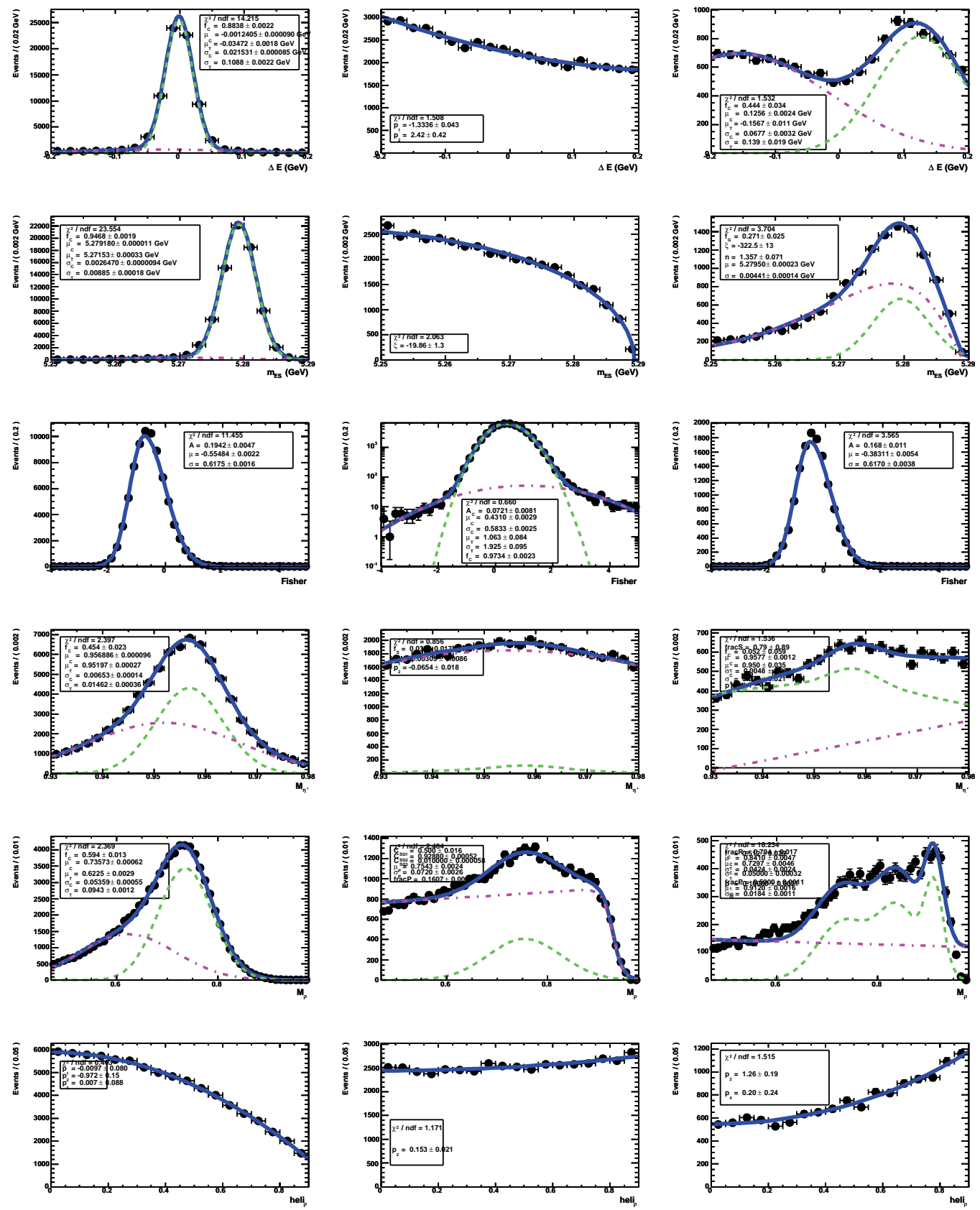

Figure C.2: PDFs for $\eta_{\rho \gamma}^{\prime} K^{+}$(from top to bottom) $\Delta E, m_{\mathrm{ES}}, \mathcal{F}, m_{\eta^{\prime}}, m_{\rho}$ and $\mathcal{H}_{\omega}$. The bottom three plots are for informational purposes only and are not variables in used in the fit. Signal MC (left), on-peak sidebands (middle) and charmless MC (right). 


$$
\begin{aligned}
& \text { C.3 } \eta_{\eta \pi \pi}^{\prime} K^{0} \\
& \text { Correlation matrix for signal eta'(epp) Ks MC (232545 events) : } \\
& \text { de mes fisher deltaT } \\
& \text { mes } \quad 0.0141 \\
& \text { fisher } \quad-0.0119 \quad-0.0130 \\
& \begin{array}{llll}
\text { deltaT } & 0.0101 & -0.0007 & -0.0039
\end{array} \\
& \begin{array}{lllll}
d t \operatorname{Err} & -0.0001 & -0.0128 & 0.0212 & -0.0158
\end{array}
\end{aligned}
$$

$\begin{array}{crrrr}\text { Correlation matrix for on-resonance data (896 events) : } & \text { de } & \text { mes } & \text { fisher deltaT } \\ \text { mes } & 0.0962 & & & \\ \text { fisher } & -0.0846 & -0.3092 & & \\ \text { deltaT } & 0.0270 & -0.0739 & 0.0218 & \\ \text { dtErr } & 0.0433 & -0.0369 & 0.0719 & -0.0838\end{array}$

Yield Fit

Floating Parameter InitialValue FinalValue (+HiError,-LoError)

$\begin{array}{rrrr}\text { deBkg_P01 } & -2.3468 \mathrm{e}+00 & -2.0710 \mathrm{e}+00(+3.05 \mathrm{e}-01,-2.99 \mathrm{e}-01) \\ \text { fisBkgC_asym } & 7.3967 \mathrm{e}-02 & 7.1180 \mathrm{e}-04(+6.42 \mathrm{e}-02,-6.33 \mathrm{e}-02) \\ \text { fisBkgC_mean } & 4.3666 \mathrm{e}-01 & 3.6452 \mathrm{e}-01 \quad(+2.41 \mathrm{e}-02,-2.42 \mathrm{e}-02) \\ \text { fisBkgC_rms } & 5.8581 \mathrm{e}-01 & 5.8727 \mathrm{e}-01 \quad(+1.86 \mathrm{e}-02,-1.80 \mathrm{e}-02) \\ \text { mesBkg_c } & -1.6667 \mathrm{e}+01 & -2.4066 \mathrm{e}+01 \quad(+9.74 \mathrm{e}+00,-9.68 \mathrm{e}+00) \\ \mathrm{nBkg} & 6.0000 \mathrm{e}+02 & 7.7065 \mathrm{e}+02(+2.95 \mathrm{e}+01,-2.87 \mathrm{e}+01) \\ \mathrm{nSig} & 2.7500 \mathrm{e}+02 & 3.2932 \mathrm{e}+02(+2.04 \mathrm{e}+01,-1.97 \mathrm{e}+01)\end{array}$


Time-dependent Fit

Floating Parameter InitialValue FinalValue (+HiError,-LoError)

\begin{tabular}{|c|c|c|c|}
\hline Frac_nBkg_tagCat_04T1 & $3.5475 e-02$ & $1.2196 \mathrm{e}-02$ & $(+4.88 e-03,-3.91 e-03)$ \\
\hline Frac_nBkg_tagCat_04T2 & $7.8619 e-02$ & $5.9612 \mathrm{e}-02$ & $(+9.44 e-03,-8.70 e-03)$ \\
\hline Frac_nBkg_tagCat_04T3 & $1.5244 \mathrm{e}-01$ & $1.5156 \mathrm{e}-01$ & $(+1.41 e-02,-1.33 e-02)$ \\
\hline Frac_nBkg_tagCat_04T4 & $1.1218 \mathrm{e}-01$ & $1.0902 \mathrm{e}-01$ & $(+1.24 e-02,-1.16 e-02)$ \\
\hline Frac_nBkg_tagCat_04T5 & $1.5340 \mathrm{e}-01$ & $1.5257 \mathrm{e}-01$ & $(+1.42 e-02,-1.35 e-02)$ \\
\hline Frac_nBkg_tagCat_04T6 & $1.0738 \mathrm{e}-01$ & $1.2239 \mathrm{e}-01$ & $(+1.28 e-02,-1.23 e-02)$ \\
\hline deBkg_P01 & $-2.3627 e+00$ & $-2.0896 e+00$ & $(+3.13 e-01,-3.05 e-01)$ \\
\hline dtBkg_fracC & $8.8334 \mathrm{e}-01$ & $9.1330 e-01$ & $(+2.48 e-02,-3.16 e-02)$ \\
\hline dtBkg_frac00 & $1.2364 \mathrm{e}-07$ & $1.9865 e-13$ & $(+2.82 e-02,--0.00 e+00)$ \\
\hline dtBkg_meanC & $1.2762 \mathrm{e}-01$ & $3.8445 e-02$ & $(+5.77 e-02,-5.78 e-02)$ \\
\hline dtBkg_meanT & $-4.5487 e-01$ & $-4.5513 e-01$ & $(+6.62 e-01,-7.49 e-01)$ \\
\hline dtBkg_sigmaC & $1.2992 \mathrm{e}+00$ & $1.3090 e+00$ & $(+5.44 e-02,-5.45 e-02)$ \\
\hline dtBkg_sigmaT & $4.0913 e+00$ & $4.3469 e+00$ & $(+8.28 e-01,-5.89 e-01)$ \\
\hline dtSig_C & $0.0000 e+00$ & $-2.5310 e-01$ & $(+1.35 e-01,-1.34 e-01)$ \\
\hline dtSig_S & $7.0000 e-01$ & $6.1343 e-01$ & $(+2.32 e-01,-2.37 e-01)$ \\
\hline nBkg & $6.0000 e+02$ & $7.2387 e+02$ & $(+2.82 e+01,-2.75 e+01)$ \\
\hline nSig & $2.7500 e+02$ & $3.1922 \mathrm{e}+02$ & $(+1.96 e+01,-1.90 e+01)$ \\
\hline
\end{tabular}



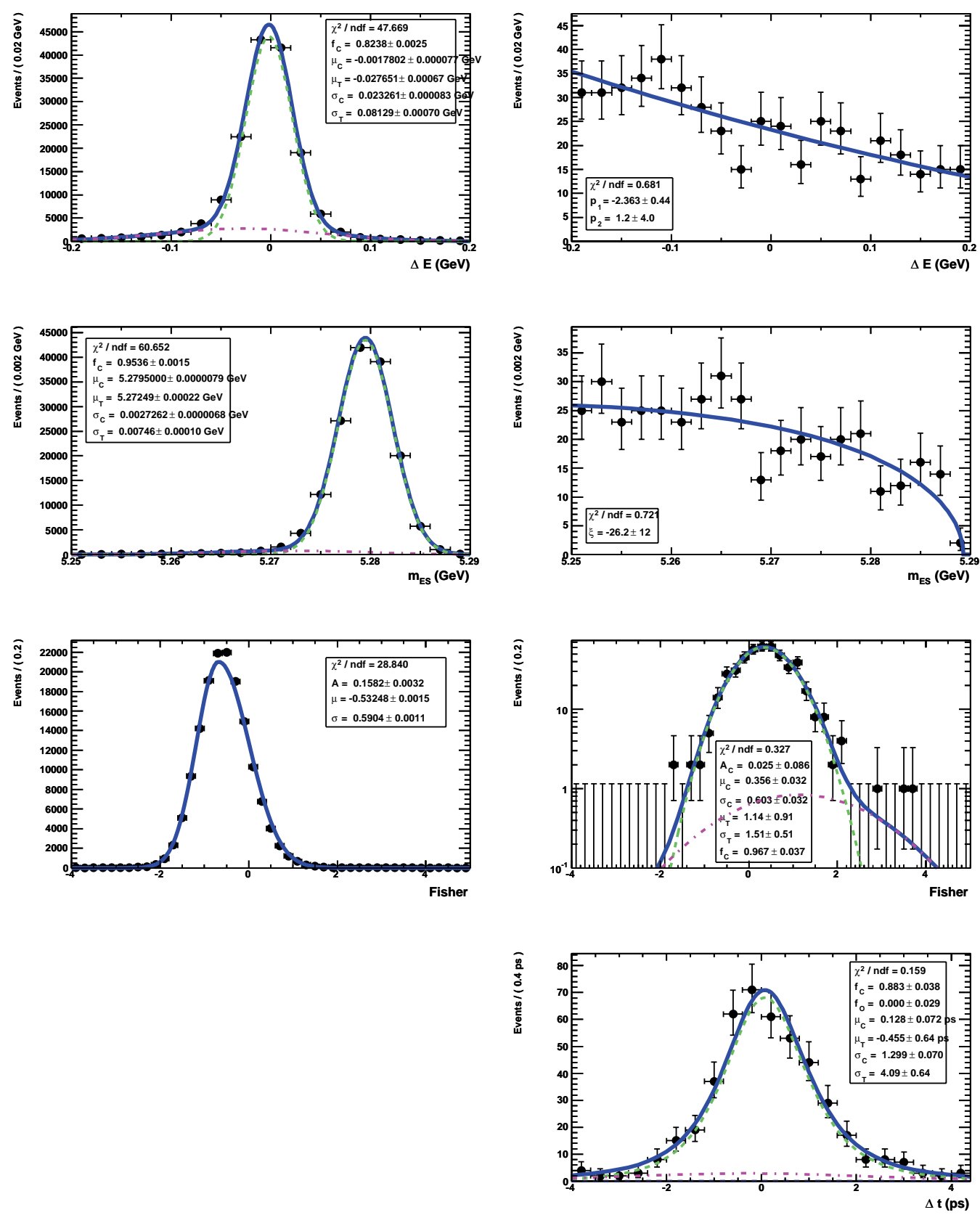

Figure C.3: PDFs for $\eta_{\eta \pi \pi}^{\prime} K^{0}$ (from top to bottom) $\Delta E, m_{\mathrm{ES}}, \mathcal{F}, \Delta t$. Signal MC (left) and on-peak sidebands (right). 


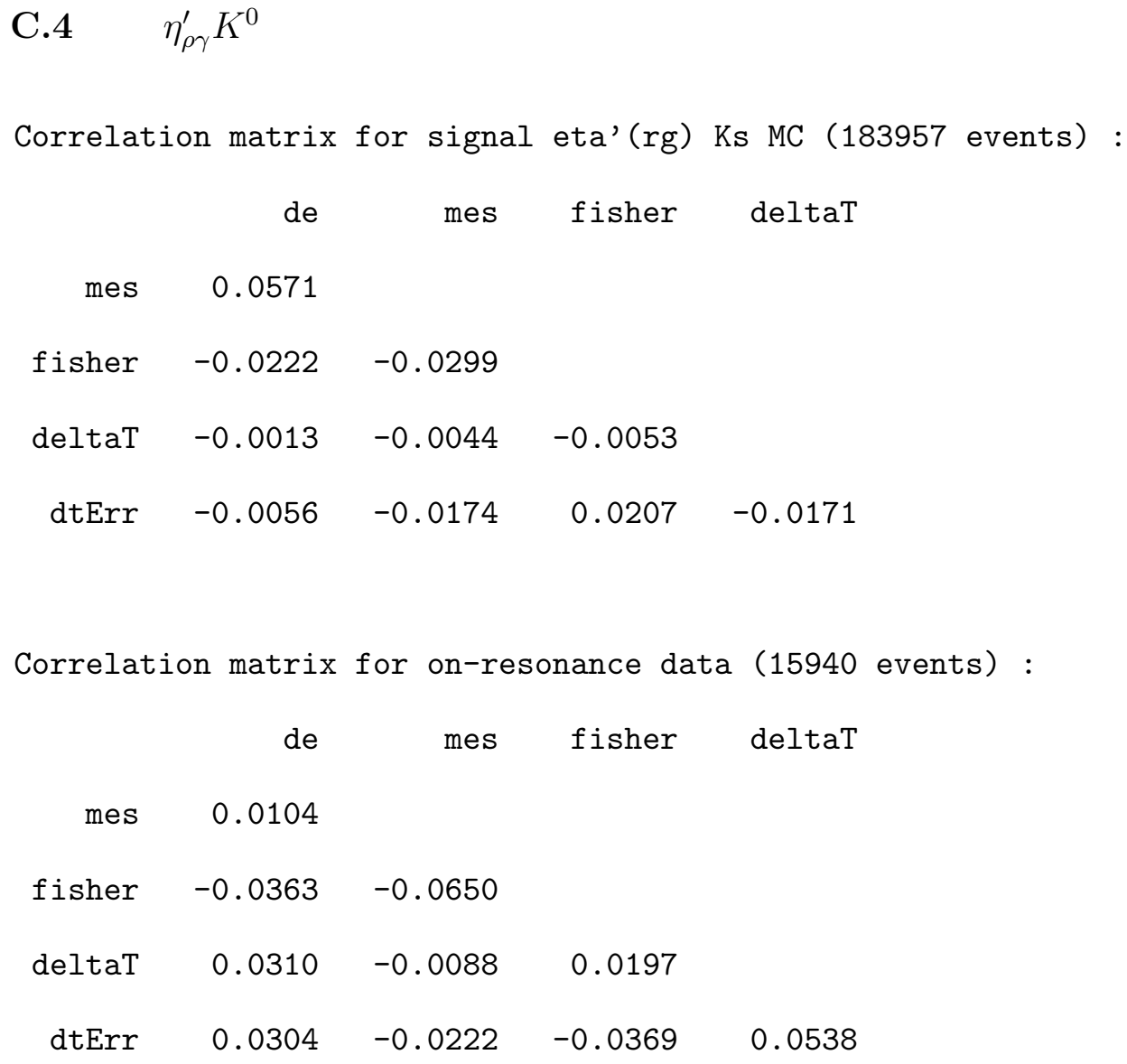

Yield Fit

Floating Parameter InitialValue FinalValue (+HiError,-LoError)

$$
\begin{array}{rrrr}
\text { deBkg_P01 } & -1.7522 \mathrm{e}+00 & -1.7300 \mathrm{e}+00(+6.39 \mathrm{e}-02,-6.38 \mathrm{e}-02) \\
\text { fisBkgC_asym } & 7.3595 \mathrm{e}-02 & 9.3060 \mathrm{e}-02(+1.34 \mathrm{e}-02,-1.33 \mathrm{e}-02) \\
\text { fisBkgC_mean } & 4.3522 \mathrm{e}-01 & 4.3762 \mathrm{e}-01(+5.46 \mathrm{e}-03,-5.45 \mathrm{e}-03) \\
\text { fisBkgC_rms } & 5.8534 \mathrm{e}-01 & 5.8869 \mathrm{e}-01(+3.84 \mathrm{e}-03,-3.82 \mathrm{e}-03) \\
\text { mesBkg_c } & -2.2384 \mathrm{e}+01 & -2.0037 \mathrm{e}+01(+2.02 \mathrm{e}+00,-2.02 \mathrm{e}+00) \\
\text { nBkg } & 1.0000 \mathrm{e}+04 & 1.8960 \mathrm{e}+04(+1.58 \mathrm{e}+02,-1.59 \mathrm{e}+02) \\
\text { nChls } & 3.0000 \mathrm{e}+02 & 1.3610 \mathrm{e}+02(+8.22 \mathrm{e}+01,-7.93 \mathrm{e}+01)
\end{array}
$$


nSig $\quad 6.6000 e+02 \quad 8.3119 e+02(+3.89 e+01,-3.81 e+01)$

Time-dependent Fit

Floating Parameter InitialValue FinalValue (+HiError,-LoError)

Frac_nBkg_tagCat_04T1

$9.2360 \mathrm{e}-03$

$4.1685 e-03(+5.62 e-04,-5.23 e-04)$

Frac_nBkg_tagCat_04T2

$8.4559 e-02$

$8.2370 e-02(+2.12 e-03,-2.09 e-03)$

Frac_nBkg_tagCat_04T3

$1.6291 \mathrm{e}-01$

$1.6221 e-01(+2.82 e-03,-2.80 e-03)$

Frac_nBkg_tagCat_04T4

$1.2288 \mathrm{e}-01$

$1.2238 e-01(+2.52 e-03,-2.49 e-03)$

Frac_nBkg_tagCat_04T5

$1.4311 \mathrm{e}-01$

$1.4254 e-01(+2.68 e-03,-2.66 e-03)$

Frac_nBkg_tagCat_04T6

$1.1550 e-01$

$1.1652 \mathrm{e}-01(+2.46 \mathrm{e}-03,-2.43 \mathrm{e}-03)$

$$
\text { deBkg_P01 }
$$

$-1.7630 e+00$

$-1.7657 e+00(+6.61 e-02,-6.58 e-02)$

dtBkg_fracC

$8.0382 e-01$

$7.9664 e-01(+2.32 e-02,-2.56 e-02)$

dtBkg_frac00

$8.8663 e-02$

$7.0718 e-02(+9.68 e-03,-8.45 e-03)$

dtBkg_meanC

$6.6293 e-02$

$5.9648 e-02(+1.34 e-02,-1.36 e-02)$

dtBkg_meanT

$8.8709 e-02$

$1.3892 e-01(+6.50 e-02,-6.52 e-02)$

dtBkg_sigmaC

$1.2182 \mathrm{e}+00$

$1.1965 e+00(+2.11 e-02,-2.20 e-02)$

dtBkg_sigmaT

$2.7289 e+00$

$2.5972 \mathrm{e}+00(+1.36 \mathrm{e}-01,-1.22 \mathrm{e}-01)$

$$
\text { dtSig_C }
$$

$0.0000 e+00$

$-2.3785 e-01(+1.01 e-01,-9.92 e-02)$

$$
\text { dtSig_S }
$$

$7.0000 e-01$

$5.3206 e-01(+1.31 e-01,-1.35 e-01)$

fisBkgC_asym

$7.5495 e-02$

$1.0820 e-01(+1.32 e-02,-1.32 e-02)$

fisBkgC_mean

$4.3390 e-01$

$4.5210 e-01(+4.95 e-03,-4.94 e-03)$

fisBkgC_rms

$5.8610 e-01$

$5.8298 e-01(+3.63 e-03,-3.59 e-03)$

mesBkg_c

$-1.9652 e+01$

$-1.5684 e+01(+1.99 e+00,-1.99 e+00)$

nBkg

$1.0000 e+04$

$1.7689 e+04(+1.40 e+02,-1.40 e+02)$

nChls

$3.0000 e+02$

$3.6112 \mathrm{e}+02(+4.94 \mathrm{e}+01,-4.74 \mathrm{e}+01)$

nSig

$6.6000 e+02$

$7.8891 e+02(+3.63 e+01,-3.56 e+01)$ 

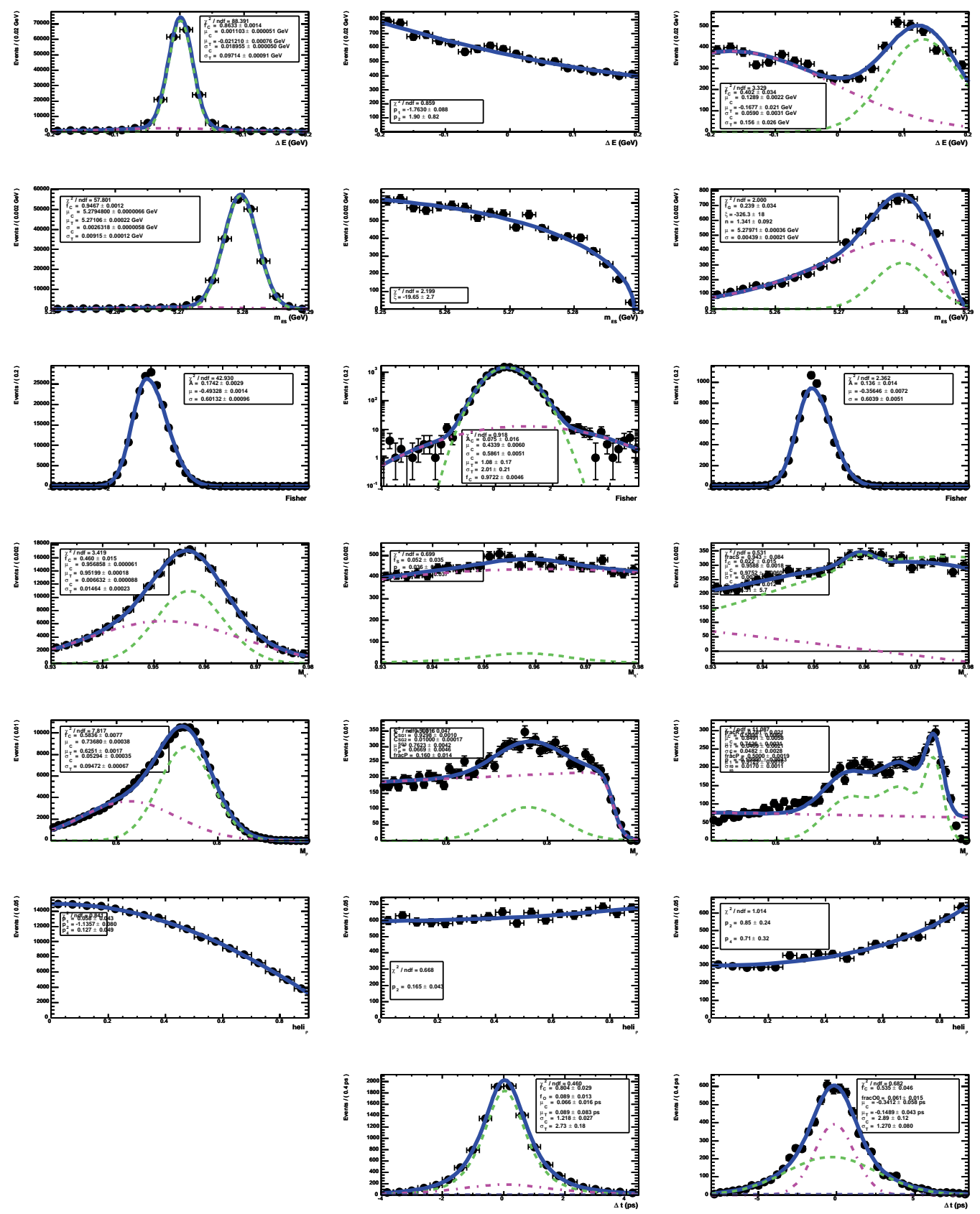

Figure C.4: PDFs for $\eta_{\rho \gamma}^{\prime} K^{0}$ (from top to bottom) $\Delta E, m_{\mathrm{ES}}, \mathcal{F}, \eta^{\prime}$ mass, $\rho$ mass, $\rho$ helicity and $\Delta t$. Signal MC (left), on-peak sidebands (middle) and $B \bar{B}$ (right). 


$$
\begin{aligned}
& \text { C.5 } \omega h^{+} \\
& \text {Correlation matrix for signal omega pi+ MC (34815 events) : } \\
& \text { de mes fisher mOmega heli pullPion } \\
& \text { mes } \quad 0.0544 \\
& \text { fisher } \quad-0.0208 \quad-0.0318 \\
& \begin{array}{llll}
\text { mOmega } & 0.2355 & 0.0150 & -0.0028
\end{array} \\
& \text { heli } \quad 0.0939 \quad 0.0748 \quad-0.0283 \quad 0.0076 \\
& \begin{array}{llllll}
\text { pullPion } & -0.0110 & 0.0024 & 0.0018 & -0.0018 & 0.0014
\end{array} \\
& \begin{array}{lllllll}
\operatorname{diffDE} & -0.0444 & -0.0102 & 0.0352 & -0.0075 & -0.0294 & 0.0052
\end{array}
\end{aligned}
$$

Correlation matrix for signal omega K+ MC (33961 events) :

$$
\begin{aligned}
& \text { de mes fisher mOmega heli pullPion } \\
& \text { mes } \quad-0.0432 \\
& \text { fisher } \quad 0.0012 \quad-0.0283 \\
& \begin{array}{llll}
\text { mOmega } & 0.2393 & -0.0014 & -0.0023
\end{array} \\
& \begin{array}{lllll}
\text { heli } & 0.0562 & 0.0713 & -0.0205 & 0.0001
\end{array} \\
& \begin{array}{llllll}
\text { pullPion } & 0.1678 & 0.0115 & -0.0419 & 0.0175 & 0.0117
\end{array} \\
& \begin{array}{lllllll}
\operatorname{diffDE} & -0.2280 & -0.0170 & 0.0239 & -0.0132 & -0.0154 & -0.7771
\end{array} \\
& \text { Correlation matrix for on-resonance data (76735 events) : } \\
& \text { de mes fisher mOmega heli pullPion } \\
& \text { mes } \quad-0.0056 \\
& \text { fisher } \quad-0.0165 \quad-0.0068 \\
& \text { mOmega } \quad-0.0026 \quad-0.0008 \quad-0.0028 \\
& \text { heli } \quad \begin{array}{llll}
-0.0030 & 0.0117 & -0.0042 & 0.0069
\end{array}
\end{aligned}
$$




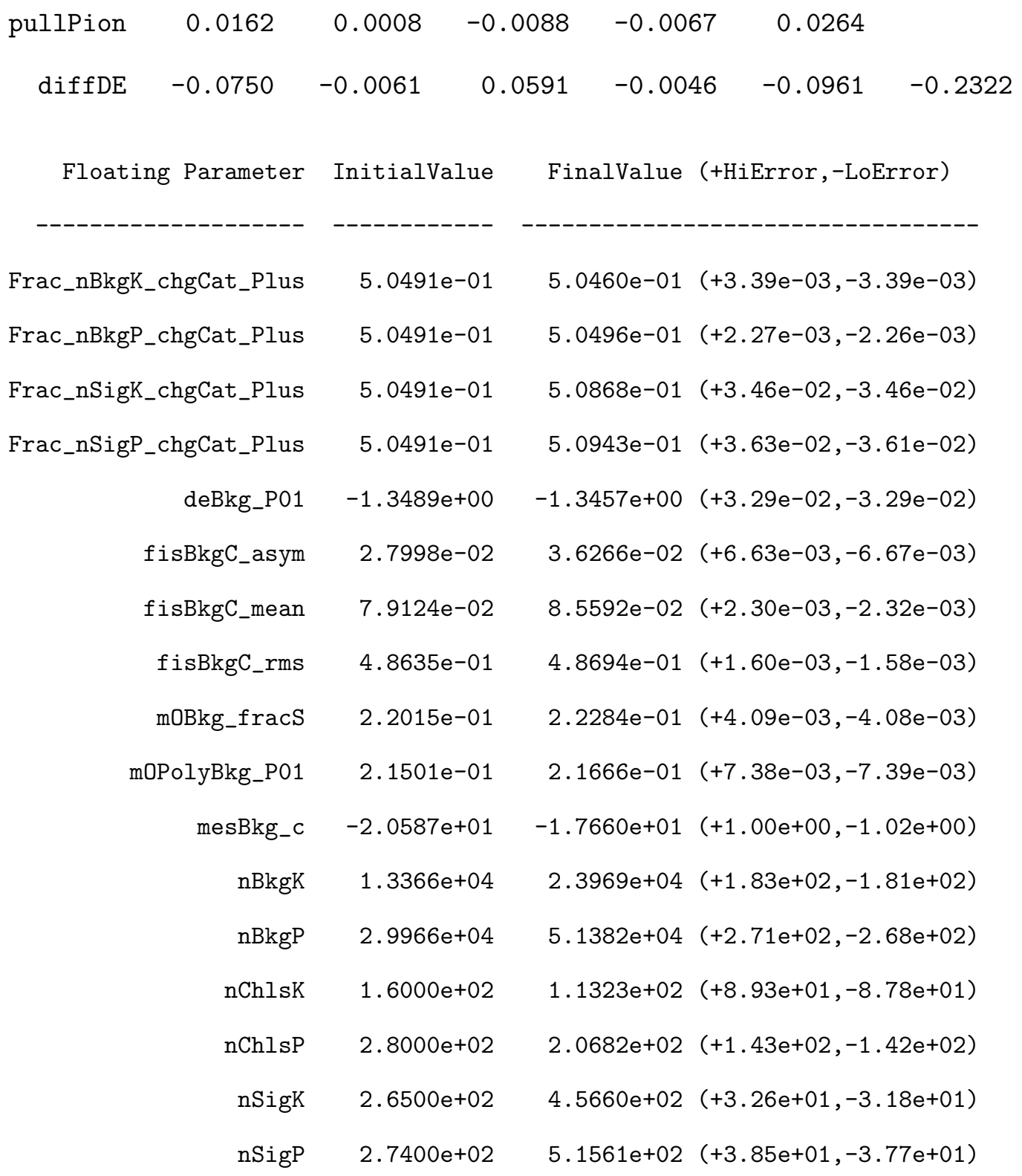



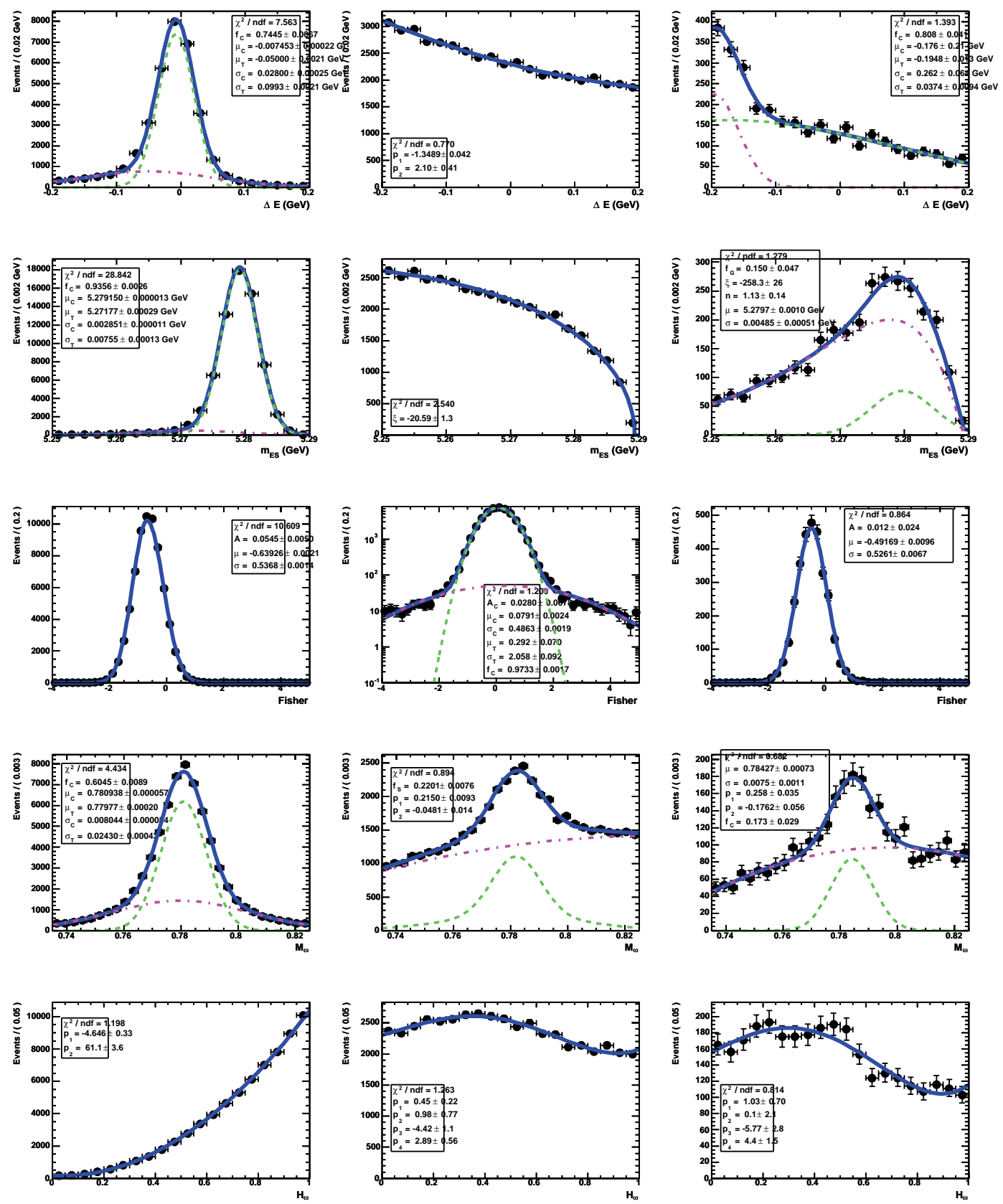

Figure C.5: PDFs for $\omega h^{+}$(from top to bottom) $\Delta E, m_{\mathrm{ES}}, \mathcal{F}, \omega$ mass, and $\omega \mathcal{H}$. Signal MC (left), on-peak sidebands (middle) and charmless MC (right). 
C.6 $\omega K_{S}^{0}$

Correlation matrix for signal MC (115517 events):

$\begin{array}{rrrrrrr} & \text { de } & \text { mes } & \text { fisher } & \text { mOmega } & \text { heli deltaT } \\ \text { mes } & 0.0790 & & & & & \\ \text { fisher } & -0.0241 & -0.0412 & & & & \\ \text { mOmega } & 0.2470 & 0.0054 & 0.0046 & & & \\ \text { heli } & 0.0981 & 0.0886 & -0.0188 & -0.0008 & & \\ \text { deltaT } & 0.0057 & -0.0045 & -0.0013 & 0.0054 & 0.0012 & \\ \text { dtErr } & -0.0145 & -0.0176 & 0.0285 & -0.0088 & -0.0204 & -0.0203\end{array}$

Correlation matrix for on-resonance data (13920 events) :

$\begin{array}{rrrrrrr} & \text { de } & \text { mes } & \text { fisher } & \text { mOmega } & \text { heli } & \text { deltaT } \\ \text { mes } & 0.0096 & & & & & \\ \text { fisher } & -0.0351 & -0.0102 & & & & \\ \text { mOmega } & -0.0072 & 0.0113 & -0.0033 & & & \\ \text { heli } & -0.0097 & 0.0142 & 0.0248 & 0.0235 & & \\ \text { deltaT } & 0.0119 & -0.0120 & 0.0068 & 0.0051 & -0.0103 & \\ \text { dtErr } & 0.0048 & -0.0260 & -0.0537 & -0.0003 & -0.0301 & 0.0310\end{array}$

Yield Fit

Floating Parameter InitialValue FinalValue (+HiError,-LoError)

deBkg_P01 $-1.5337 \mathrm{e}+00 \quad-1.4032 \mathrm{e}+00(+6.99 \mathrm{e}-02,-6.96 \mathrm{e}-02)$

fisBkgC_asym $1.1476 \mathrm{e}-01 \quad 1.1415 \mathrm{e}-01(+1.38 \mathrm{e}-02,-1.39 \mathrm{e}-02)$

fisBkgC_mean $\quad 4.2263 e-01 \quad 4.3017 \mathrm{e}-01(+4.94 \mathrm{e}-03,-4.95 \mathrm{e}-03)$

fisBkgC_rms $\quad 5.6942 \mathrm{e}-01 \quad 5.7311 \mathrm{e}-01(+3.66 \mathrm{e}-03,-3.67 \mathrm{e}-03)$

heliBkg_P01 $\quad 4.0259 \mathrm{e}-01 \quad 3.2536 \mathrm{e}-01(+1.17 \mathrm{e}-01,-1.13 \mathrm{e}-01)$

heliBkg_P02 $-6.0769 \mathrm{e}-01 \quad-5.4652 \mathrm{e}-01(+1.11 \mathrm{e}-01,-1.15 \mathrm{e}-01)$ 


$\begin{array}{rrr}\text { mOBkg_fracS } & 1.9385 \mathrm{e}-01 & 1.8349 \mathrm{e}-01(+8.78 \mathrm{e}-03,-8.75 \mathrm{e}-03) \\ \text { m0PolyBkg_P01 } & 1.8323 \mathrm{e}-01 & 2.0064 \mathrm{e}-01(+1.57 \mathrm{e}-02,-1.58 \mathrm{e}-02) \\ \text { mesBkg_c } & -1.9043 \mathrm{e}+01 & -1.7967 \mathrm{e}+01(+2.06 \mathrm{e}+00,-2.05 \mathrm{e}+00) \\ \mathrm{nBkg} & 1.0000 \mathrm{e}+04 & 1.5724 \mathrm{e}+04(+1.27 \mathrm{e}+02,-1.26 \mathrm{e}+02) \\ \mathrm{nSig} & 1.5000 \mathrm{e}+02 & 1.4551 \mathrm{e}+02(+1.85 \mathrm{e}+01,-1.77 \mathrm{e}+01)\end{array}$

Time-dependent Fit

Floating Parameter InitialValue

FinalValue (+HiError,-LoError)

\begin{tabular}{|c|c|c|}
\hline Frac_nBkg_tagCat_04T1 & $6.1780 e-03$ & $4.6584 e-03(+6.41 e-04,-5.90 e-04)$ \\
\hline Frac_nBkg_tagCat_04T2 & $8.2687 e-02$ & $8.1997 e-02(+2.37 e-03,-2.33 e-03)$ \\
\hline Frac_nBkg_tagCat_04T3 & $1.5575 e-01$ & $1.5578 e-01(+3.14 e-03,-3.07 e-03)$ \\
\hline Frac_nBkg_tagCat_04T4 & $1.1688 \mathrm{e}-01$ & $1.1650 \mathrm{e}-01(+2.76 \mathrm{e}-03,-2.74 \mathrm{e}-03)$ \\
\hline Frac_nBkg_tagCat_04T5 & $1.4612 \mathrm{e}-01$ & $1.4636 \mathrm{e}-01(+3.04 \mathrm{e}-03,-3.01 \mathrm{e}-03)$ \\
\hline Frac_nBkg_tagCat_04T6 & $1.1401 \mathrm{e}-01$ & $1.1407 e-01(+2.75 e-03,-2.70 e-03)$ \\
\hline deBkg_P01 & $-1.5337 e+00$ & $-1.4557 e+00(+7.48 e-02,-7.45 e-02)$ \\
\hline dtBkg_fracC & $8.9360 e-01$ & $8.9619 e-01(+4.78 e-03,-5.01 e-03)$ \\
\hline dtBkg_frac00 & $7.5254 \mathrm{e}-02$ & $8.0556 e-02(+2.67 e-02,-2.39 e-02)$ \\
\hline dtBkg_meanC & $4.9775 e-02$ & $5.3162 e-02(+1.28 e-02,-1.28 e-02)$ \\
\hline dtBkg_meanT & $1.1146 \mathrm{e}+00$ & $9.5985 e-01(+3.12 e-01,-3.02 e-01)$ \\
\hline dtBkg_sigmaC & $1.3240 e+00$ & $1.3051 e+00(+1.28 e-02,-1.28 e-02)$ \\
\hline dtBkg_sigmaT & $8.1872 \mathrm{e}+00$ & $8.3588 e+00(+5.29 e-01,-5.09 e-01)$ \\
\hline dtSig_C & $0.0000 e+00$ & $-3.9045 e-01(+2.40 e-01,-2.32 e-01)$ \\
\hline dtSig_S & $7.0000 e-01$ & $6.3102 e-01(+2.33 e-01,-2.75 e-01)$ \\
\hline fisBkgC_asym & $1.1476 \mathrm{e}-01$ & $1.0897 e-01(+1.48 e-02,-1.48 e-02)$ \\
\hline fisBkgC_mean & $4.2263 e-01$ & $4.2994 e-01(+5.37 e-03,-5.36 e-03)$ \\
\hline fisBkgC_rms & $5.6942 e-01$ & $5.7214 e-01(+3.97 e-03,-3.94 e-03)$ \\
\hline
\end{tabular}




$\begin{array}{rrr}\text { heliBkg_P01 } & 4.0259 \mathrm{e}-01 & 3.9338 \mathrm{e}-01(+1.30 \mathrm{e}-01,-1.24 \mathrm{e}-01) \\ \text { heliBkg_P02 } & -6.0769 \mathrm{e}-01 & -5.9931 \mathrm{e}-01(+1.22 \mathrm{e}-01,-1.27 \mathrm{e}-01) \\ \text { m0Bkg_fracS } & 1.9385 \mathrm{e}-01 & 2.0049 \mathrm{e}-01(+9.39 \mathrm{e}-03,-9.37 \mathrm{e}-03) \\ \text { mOPolyBkg_P01 } & 1.8323 \mathrm{e}-01 & 2.0265 \mathrm{e}-01(+1.70 \mathrm{e}-02,-1.70 \mathrm{e}-02) \\ \text { mesBkg_c } & -1.9043 \mathrm{e}+01 & -1.7812 \mathrm{e}+01(+2.21 \mathrm{e}+00,-2.21 \mathrm{e}+00) \\ \text { nBkg } & 1.0000 \mathrm{e}+04 & 1.3735 \mathrm{e}+04(+1.20 \mathrm{e}+02,-1.19 \mathrm{e}+02) \\ \mathrm{nChls} & 1.0000 \mathrm{e}+01 & 3.6047 \mathrm{e}+01(+2.63 \mathrm{e}+01,-2.20 \mathrm{e}+01) \\ \mathrm{nSig} & 9.6000 \mathrm{e}+01 & 1.4952 \mathrm{e}+02(+1.73 \mathrm{e}+01,-1.63 \mathrm{e}+01)\end{array}$



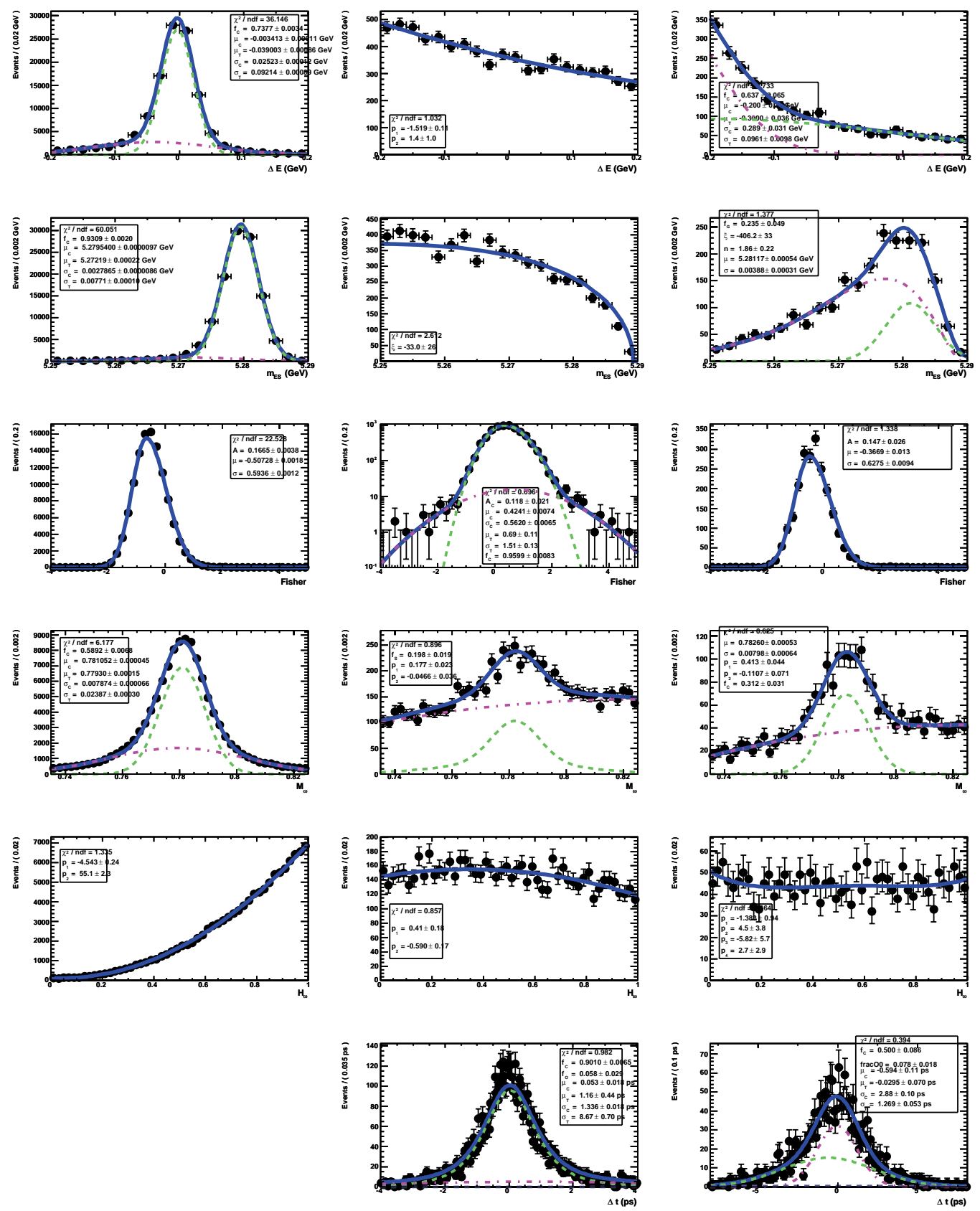

Figure C.6: PDFs for $\omega K_{S}^{0}$ (from top to bottom) $\Delta E, m_{\mathrm{ES}}, \mathcal{F}, \omega$ mass, $\omega \mathcal{H}$, and $\Delta t$. Signal MC (left), on-peak sidebands (middle) and $B \bar{B}$ (right). 


\section{Appendix D}

\section{Fit Variable Correlations}

The maximum likelihood fit relies on the assumption that the variables in the fit are uncorrelated. To investigate this beyond linear correlation coefficients we show "profile" plots of the fit variables for $\eta_{\rho \gamma}^{\prime} K^{+}$in Fig. D.1. The plots have been made from on-peak sidebands to remove signal events. Generally, the correlations are quite small. Fig. D.2 shows the same correlation profile plots with a restricted range on each plot.

The correlation between $\Delta E$ and $\Delta t / \sigma(\Delta t)$ shows a variation of $\sim 30 \mathrm{MeV}$ over the 4 central bins in $\Delta t / \sigma(\Delta t)$ (middle column, bottom row of Fig. D.1). To evaluate the effect of this correlation on our fit, we split the data into different regions of $\Delta t$ and fit the background $\Delta E$ shapes in each of these regions separately. Accounting for the correlations in this way, we found the fit result changed by less than 1 event and that $\mathrm{S}$ and $\mathrm{C}$ both changed by less than 0.002 compared to the unsplit fit. A physical reason for this correlation is still unknown, but a similar structure with a smaller total

variation in $\Delta E$ was also observed in $B \rightarrow \omega K_{S}^{0}$ and $B \rightarrow \pi \pi$. Based on these studies we conclude that any potential biases in the fit due to correlations in the background are small. 

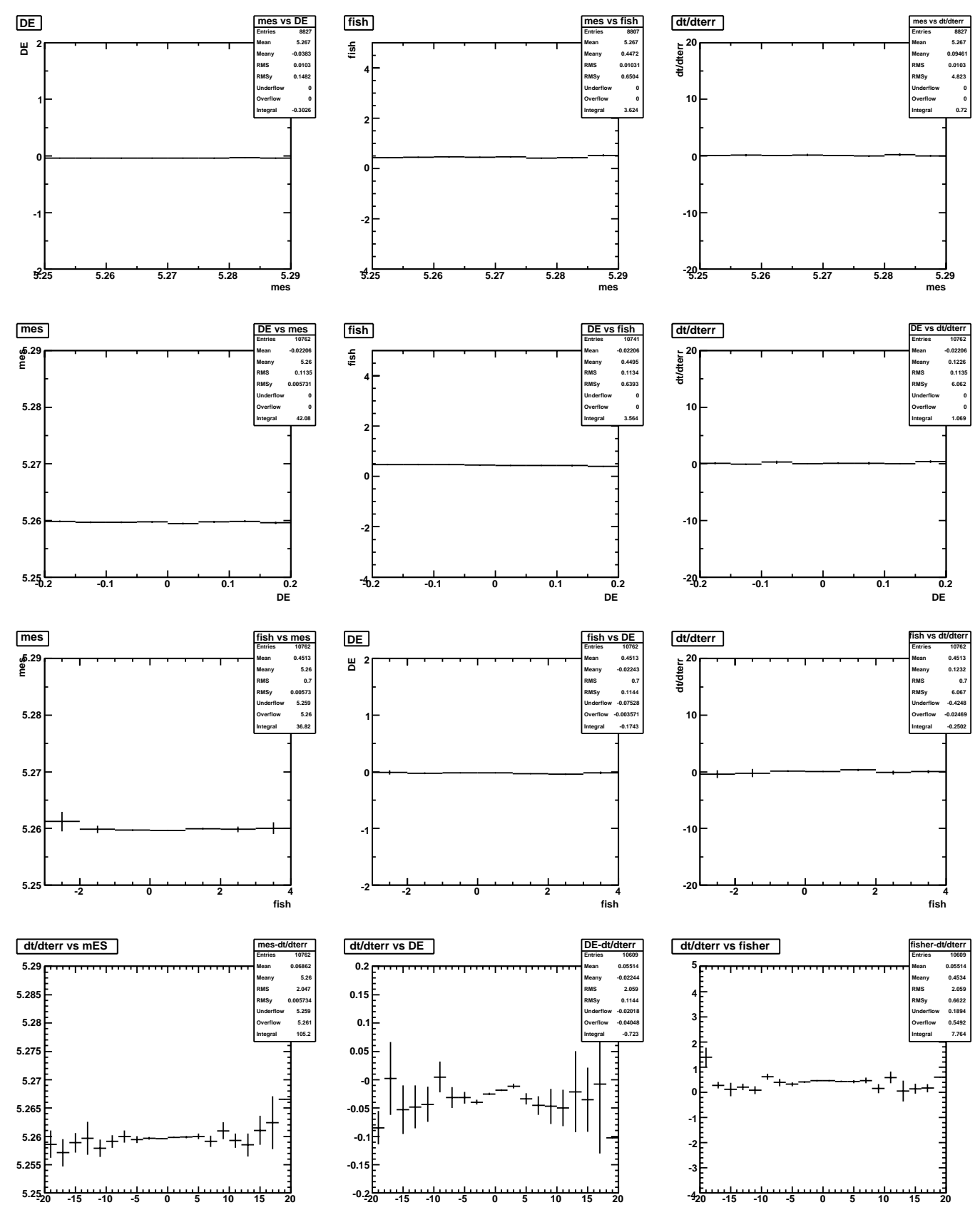

Figure D.1: Profile plots from on-peak data with signal removed for $\eta_{\rho \gamma}^{\prime} K^{+}$for each combination of $\Delta E, m_{\mathrm{ES}}, \mathcal{F}$ and $\Delta t / \sigma(\Delta t)$ as labeled. 

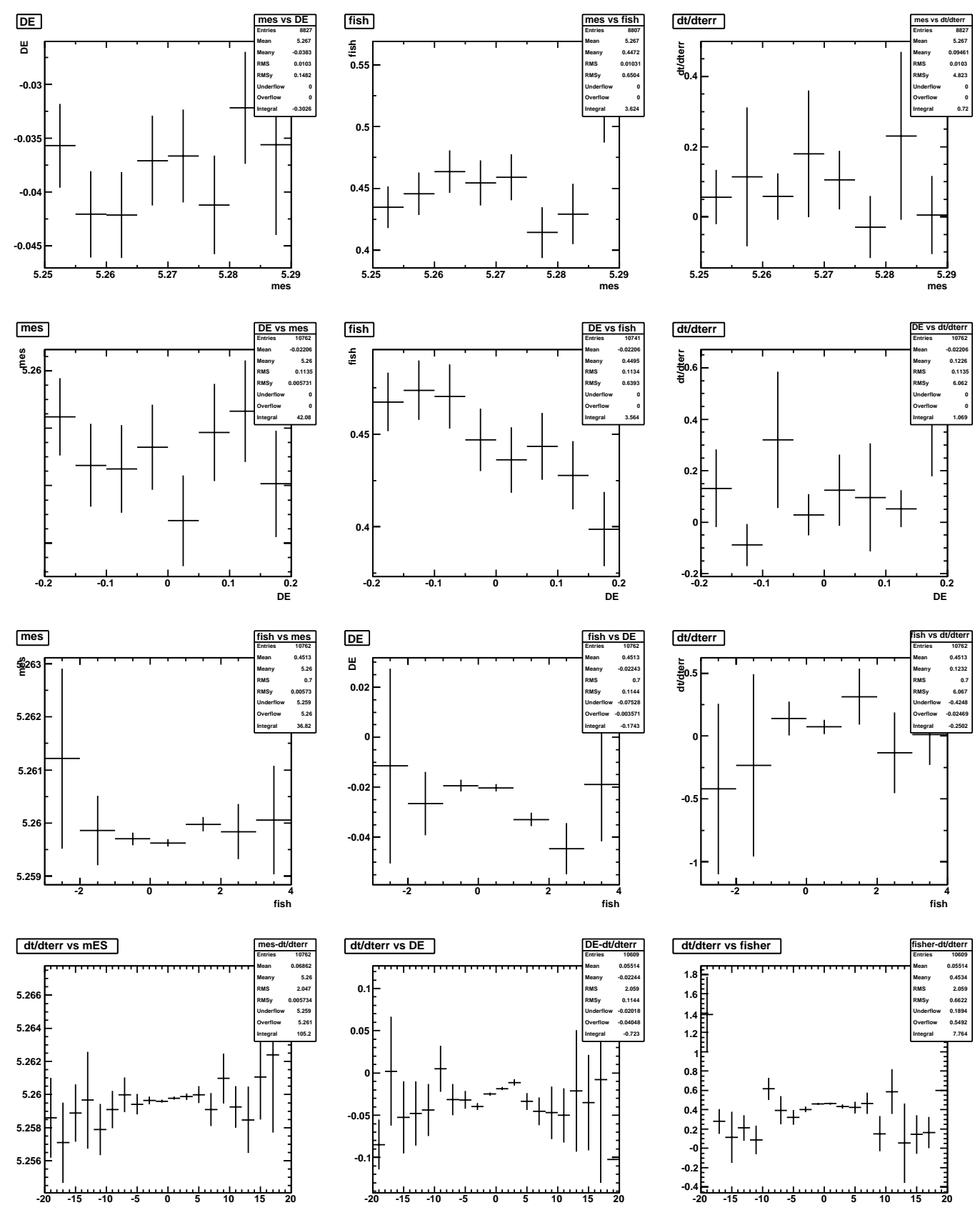

Figure D.2: Profile plots from on-peak data with signal removed for $\eta_{\rho \gamma}^{\prime} K^{+}$for each combination of $\Delta E, m_{\mathrm{ES}}, \mathcal{F}$ and $\Delta t / \sigma(\Delta t)$ as labeled. 


\section{Appendix E}

\section{Pure Toy Pulls}

We show here the pulls from pure toys for the floating parameters in the fits.

Table E.1: Pulls from floating parameters in 1000 pure toy experiments for $\eta_{\eta \pi \pi}^{\prime} K^{+}$ yield fits.

$\begin{array}{rcc} & \text { mean } & \text { sigma } \\ \text { deBkg_P01 } & -0.03+/-0.03 & 0.96+/-0.02 \\ \text { fisBkgC_mean } & 0.03+/-0.03 & 0.99+/-0.02 \\ \text { fisBkgC_asym } & 0.00+/-0.03 & 0.95+/-0.02 \\ \text { fisBkgC_rms } & -0.08+/-0.03 & 1.00+/-0.03 \\ \text { nBkg } & -0.01+/-0.03 & 1.00+/-0.02 \\ \text { nSig } & -0.01+/-0.03 & 1.03+/-0.02\end{array}$

Table E.2: Pulls from floating parameters in 1000 pure toy experiments for $\eta_{\rho \gamma}^{\prime} K^{+}$yield fits.

$\begin{array}{rrc} & \text { mean } & \text { sigma } \\ \text { deBkg_P01 } & 0.06+/-0.03 & 1.01+/-0.02 \\ \text { fisBkgC_mean } & -0.13+/-0.03 & 0.95+/-0.02 \\ \text { fisBkgC_asym } & -0.07+/-0.03 & 1.03+/-0.03 \\ \text { fisBkgC_rms } & 0.09+/-0.03 & 0.95+/-0.02 \\ \text { mesBkg_c } & -0.07+/-0.03 & 0.97+/-0.02 \\ \text { nBkg } & 0.11+/-0.03 & 1.01+/-0.03 \\ \text { nChls } & -0.20+/-0.03 & 1.00+/-0.03 \\ \text { nSig } & 0.06+/-0.03 & 1.00+/-0.02\end{array}$


Table E.3: Pulls from floating parameters in 1000 pure toy experiments for $\eta_{\eta \pi \pi}^{\prime} K_{S}^{0}$ yield fits.

$\begin{array}{rrc} & \text { mean } & \text { sigma } \\ \text { deBkg_P01 } & 0.03+/-0.03 & 0.99+/-0.03 \\ \text { fisBkgC_mean } & -0.02+/-0.03 & 0.94+/-0.02 \\ \text { fisBkgC_asym } & -0.03+/-0.03 & 0.99+/-0.02 \\ \text { fisBkgC_rms } & -0.07+/-0.03 & 0.95+/-0.03 \\ \text { nBkg } & 0.02+/-0.03 & 0.98+/-0.02 \\ \text { nSig } & -0.04+/-0.03 & 1.00+/-0.02\end{array}$

Table E.4: Pulls from floating parameters in 1000 pure toy experiments for $\eta_{\rho \gamma}^{\prime} K_{S}^{0}$ yield fits.

$\begin{array}{rrc} & \text { mean } & \text { sigma } \\ \text { deBkg_P01 } & -0.00+/-0.03 & 0.98+/-0.02 \\ \text { fisBkgC_mean } & -0.05+/-0.03 & 0.99+/-0.03 \\ \text { fisBkgC_asym } & -0.02+/-0.03 & 0.96+/-0.02 \\ \text { fisBkgC_rms } & 0.02+/-0.03 & 1.02+/-0.03 \\ \text { mesBkg_c } & -0.01+/-0.03 & 0.98+/-0.02 \\ \text { nBkg } & 0.06+/-0.03 & 0.98+/-0.02 \\ \text { nChls } & -0.09+/-0.03 & 0.99+/-0.03 \\ \text { nSig } & 0.04+/-0.03 & 0.98+/-0.02\end{array}$


Table E.5: Pulls from floating parameters in 1000 pure toy experiments for $\omega \pi^{+}$and $\omega K^{+}$yield fits.

$\begin{array}{rrc} & \text { mean } & \text { sigma } \\ \text { deBkg_P01 } & -0.08+/-0.04 & 1.00+/-0.03 \\ \text { fisBkgC_mean } & -0.18+/-0.03 & 0.91+/-0.03 \\ \text { fisBkgC_asym } & -0.10+/-0.04 & 0.99+/-0.03 \\ \text { fisBkgC_rms } & 0.10+/-0.04 & 0.94+/-0.03 \\ \text { mesBkg_c } & -0.12+/-0.04 & 1.00+/-0.03 \\ \text { mOBkg_fracS } & 0.04+/-0.04 & 1.01+/-0.03 \\ \text { mOPolyBkg_P01 } & 0.05+/-0.04 & 0.97+/-0.03 \\ \text { nBkgK } & 0.09+/-0.04 & 0.94+/-0.03 \\ \text { nBkgP } & 0.09+/-0.04 & 0.95+/-0.03 \\ \text { nChlsK } & -0.23+/-0.04 & 0.98+/-0.03 \\ \text { nChlsP } & -0.21+/-0.11 & 0.81+/-0.10 \\ \text { nSigK } & 0.17+/-0.04 & 1.01+/-0.03 \\ \text { nSigP } & -0.01+/-0.04 & 1.00+/-0.03\end{array}$

Table E.6: Pulls from floating parameters in 1000 pure toy experiments for $\omega K_{S}^{0}$ yield fits.

$\begin{array}{rcc} & \text { mean } & \text { sigma } \\ \text { deBkg_P01 } & 0.03+/-0.03 & 0.98+/-0.02 \\ \text { fisBkgC_mean } & 0.10+/-0.03 & 1.00+/-0.02 \\ \text { fisBkgC_asym } & 0.09+/-0.03 & 0.89+/-0.02 \\ \text { fisBkgC_rms } & -0.04+/-0.03 & 0.96+/-0.02 \\ \text { mesBkg_c } & -0.01+/-0.03 & 1.00+/-0.02 \\ \text { mOBkg_fracS } & 0.03+/-0.03 & 1.02+/-0.02 \\ \text { mOPolyBkg_P01 } & 0.02+/-0.03 & 0.95+/-0.02 \\ \text { nBkg } & -0.05+/-0.03 & 1.01+/-0.02 \\ \text { nSig } & -0.06+/-0.03 & 0.94+/-0.02\end{array}$


Table E.7: Pulls from floating parameters in 500 pure toy experiments for $\eta_{\eta \pi \pi}^{\prime} K^{0}$ generated with our best fit values of $C=-0.25$ and $S=0.6$ in $\Delta t$ fits.

$\begin{array}{rrc} & \text { mean } & \text { sigma } \\ \text { deBkg_P01 } & -0.04+/-0.04 & 0.96+/-0.03 \\ \text { dtBkg_fracC } & 0.19+/-0.05 & 1.03+/-0.03 \\ \text { dtBkg_frac00 } & 0.12+/-0.01 & 0.20+/-0.01 \\ \text { dtBkg_meanC } & 0.01+/-0.05 & 1.01+/-0.03 \\ \text { dtBkg_meanT } & -0.03+/-0.04 & 0.99+/-0.03 \\ \text { dtBkg_sigmaC } & 0.04+/-0.04 & 0.95+/-0.03 \\ \text { dtBkg_sigmaT } & 0.03+/-0.05 & 1.05+/-0.03 \\ \text { dtSig_C } & -0.02+/-0.04 & 0.99+/-0.03 \\ \text { dtSig_S } & 0.00+/-0.05 & 1.07+/-0.03 \\ \text { fisBkgC_mean } & 0.04+/-0.05 & 1.03+/-0.03 \\ \text { fisBkgC_asym } & 0.01+/-0.05 & 1.02+/-0.03 \\ \text { fisBkgC_rms } & -0.05+/-0.05 & 1.06+/-0.03 \\ \text { nBkg } & -0.01+/-0.04 & 0.96+/-0.03 \\ \text { nSig } & 0.10+/-0.04 & 0.95+/-0.03\end{array}$

Table E.8: Pulls from floating parameters in 500 pure toy experiments for $\eta_{\rho \gamma}^{\prime} K^{0}$ generated with our best fit values of $C=-0.3$ and $S=0.5$ in $\Delta t$ fits.

$\begin{array}{rrc} & \text { mean } & \text { sigma } \\ \text { deBkg_P01 } & 0.02+/-0.05 & 1.05+/-0.04 \\ \text { dtBkg_fracC } & -0.01+/-0.05 & 0.94+/-0.03 \\ \text { dtBkg_frac00 } & 0.04+/-0.04 & 0.93+/-0.04 \\ \text { dtBkg_meanC } & -0.02+/-0.05 & 1.00+/-0.04 \\ \text { dtBkg_meanT } & 0.10+/-0.04 & 0.92+/-0.03 \\ \text { dtBkg_sigmaC } & 0.02+/-0.04 & 0.94+/-0.04 \\ \text { dtBkg_sigmaT } & 0.10+/-0.05 & 0.97+/-0.04 \\ \text { dtSig_C } & -0.01+/-0.05 & 0.95+/-0.03 \\ \text { dtSig_S } & 0.05+/-0.05 & 1.03+/-0.04 \\ \text { fisBkgC_mean } & -0.06+/-0.04 & 0.96+/-0.04 \\ \text { fisBkgC_asym } & 0.02+/-0.05 & 0.97+/-0.04 \\ \text { fisBkgC_rms } & -0.08+/-0.04 & 0.93+/-0.03 \\ \text { mesBkg_c } & -0.02+/-0.04 & 0.90+/-0.03 \\ \text { nBkg } & -0.07+/-0.04 & 0.93+/-0.04 \\ \text { nChls } & -0.13+/-0.05 & 1.00+/-0.04 \\ \text { nSig } & 0.09+/-0.05 & 0.97+/-0.03\end{array}$


Table E.9: Pulls from floating parameters in 3000 pure toy experiments for $\omega K_{S}^{0}$ generated with our best fit values of $C=-0.43$ and $S=0.62$ in $\Delta t$ fits.

$\begin{array}{rrc} & \text { mean } & \text { sigma } \\ \text { deBkg_P01 } & -0.04+/-0.02 & 1.00+/-0.01 \\ \text { dtSig_C } & -0.03+/-0.02 & 1.05+/-0.01 \\ \text { dtSig_S } & 0.08+/-0.02 & 1.05+/-0.01 \\ \text { fisBkgC_mean } & -0.06+/-0.02 & 0.99+/-0.01 \\ \text { fisBkgC_asym } & -0.06+/-0.02 & 0.98+/-0.01 \\ \text { fisBkgC_rms } & 0.04+/-0.02 & 1.01+/-0.01 \\ \text { mesBkg_c } & -0.05+/-0.02 & 0.99+/-0.01 \\ \text { mOBkg_fracS } & 0.01+/-0.02 & 0.98+/-0.01 \\ \text { mOPolyBkg_P01 } & 0.03+/-0.02 & 0.99+/-0.01 \\ \text { nBkg } & 0.04+/-0.02 & 0.97+/-0.01 \\ \text { nSig } & 0.02+/-0.02 & 0.99+/-0.01\end{array}$




\section{Appendix F}

\section{Combining Results with Log Likelihood Curves}

Branching fraction and asymmetry results from different decay sub-modes are combined using their log-likelihood curves. These curves are adjusted to consider the effects of systematic uncertainties. Four types of systematic uncertainties are considered:

- Additive Systematic Uncertainties affect the central value and thus the statistical significance of a result. For example, a bias in the maximum likelihood fitter could systematically increase or decrease the number of events reported.

- Multiplicative Systematic Uncertainties don't affect the significance of the result.

- Uncorrelated Systematic Uncertainties are mode-independent. For example, uncertainties in the daughter particle branching fractions affect the conversion of the fit yield into a branching fraction, but not the statistical significance of the fit yield itself.

- Correlated Systematic Uncertainties affect all modes in the same direction. For example, a Monte Carlo PID selection efficiency correction could bias all modes which use the selector up or down.

Each systematic error is either additive or multiplicative, and either correlated or uncorrelated. Additive systematic uncertainties are included in the quoted statistical significance of individual and combined results. The final combined branching fractions and asymmetries include both uncorrelated and correlated systematic uncertainties. 


\section{F.1 Log Likelihood Curve Basics}

If a measured quantity with mean $\mu$ has Gaussian errors, its likelihood will be of the form:

$$
\mathcal{L}=\mathcal{L}_{\text {max }} \exp \left[-\frac{(x-\mu)^{2}}{2 \sigma^{2}}\right] .
$$

Thus the $\log$-likelihood $\chi^{2} \equiv-2 \ln \left(\mathcal{L} / \mathcal{L}_{\text {max }}\right)=(x-\mu)^{2} / \sigma^{2}$ is a parabola which crosses 1 at $\mu \pm \sigma, 4$ at $\mu \pm 2 \sigma$, etc. A measure of the significance of a result is the square root of the value $-2 \ln \left(\mathcal{L} / \mathcal{L}_{\text {max }}\right)$ at $x=0$.

Statistically combining results is equivalent to multiplying likelihood curves, i.e., adding the log-likelihood curves and adjusting the minimum back to 0 . This procedure applies even if the likelihood curves are asymmetric or otherwise non-Gaussian.

Including a systematic uncertainty $\sigma_{\text {syst }}$ involves convoluting the likelihood with a Gaussian of width $\sigma_{\text {syst }}$. If the original likelihood curve is Gaussian with width $\sigma_{\text {stat }}$, this produces a new Gaussian likelihood curve with $\sigma^{2}=\sigma_{\text {syst }}^{2}+\sigma_{\text {stat }}^{2}$. Equivalently, this adjusts the log-likelihood curve by:

$$
\chi^{2}=\frac{\chi_{\text {stat }}^{2} \chi_{\text {syst }}^{2}}{\chi_{\text {stat }}^{2}+\chi_{\text {syst }}^{2}}
$$

where $\chi_{\text {stat }}^{2}=(x-\mu)^{2} / \sigma_{\text {stat }}^{2}$ and similarly for $\chi_{\text {syst }}^{2}$. This has the effect of broadening the log-likelihood curve to account for the systematic uncertainty.

\section{F.2 Statistical Significance with Systematics}

To assess the statistical significance with systematics of each mode, the individual log-likelihood curves for the fit yield $N$ are corrected using equation F.2. For asymmetric curves, this is an approximation of the effect of including the systematic uncertainties. The corrected curves are added together and the minimum of the combined curve is readjusted to 0 . The significance of the combined result is $\sqrt{-2 \ln \left(\mathcal{L} / \mathcal{L}_{\text {max }}\right)}$ at $N=0$. 


\section{F.3 Combining Branching Fractions and Asymmetries with Sys- tematics}

We produce likelihood scan curves from RooRarFit refitting the data at each point in the scan. Uncorrelated systematic uncertainties are included by applying equation F.2 to the log-likelihood curve of each mode. The combined result with uncorrelated systematic errors is the sum of these corrected log-likelihood curves with the value of the combined $\ln (\mathcal{L})$ at the minimum adjusted back to zero.

Correlated systematic uncertainties shift all curves up or down together so they should not be applied to the individual curves before combining. The correlated systematics for each mode might be different, but they cause a bias for all curves in the same direction and thus have a different effect on the combined log-likelihood curve. To account for correlated systematic uncertainties, the curves for the individual modes are shifted down by one sigma and the mean of the combined curve is found. This is repeated while shifting the individual curves up by one sigma. The average change in the combined mean is the weighted correlated systematic uncertainty. This uncertainty is applied to the combined log-likelihood curve in the same way as the uncorrelated uncertainties described above.

This procedure for finding combined results by adjusting log-likelihood curves reduces to the standard covariance matrix formulation when the original errors are simply Gaussian (i.e., the log-likelihood curves are parabolas). Our procedure is appropriate in cases where the errors are asymmetric or the likelihood curves are otherwise nonGaussian.

\section{F.4 The combine.cc Script}

The likelihood curves for each mode are output in a RooPlot object by the RooRarFit code. These curves are combined with a Root script which can combine 
an arbitrary number of modes including various systematic errors as described above. The script and its documentation are available as a part of the RooRarFit fitting package. 


\section{Appendix G}

\section{Overlap Bug Effect}

In release 18.2.1, reconstruction code was improved to allow a single calorimeter cluster to be matched with two different tracks within a candidate. This improvement revealed a latent bug in BetaRecoAdapter V00-10-01 (and earlier) in which the BtaRecoPointers::overlaps() function considered two tracks matched to a single cluster to "overlap" and rejected the related candidate. The overlaps function was changed in BetaRecoAdapter V00-11-01 so that it would not automatically reject these candidates.

We have found that $33 \%$ of our data and none of our MC had the original event reconstruction done with a release earlier than 18.2.1. Additionally, $100 \%$ of our data and MC were further processed with the incorrect BetaRecoAdapter V00-10-01. Thus, the detection efficiency obtained from MC is inappropriate for $33 \%$ of the data. To quantify this data/MC difference we ran two jobs on unskimmed MC, one with BetaRecoAdapter V00-10-01 and one with V00-11-01. We compare the efficiencies obtained from these two job configurations in Table G.1.

The effect of the bug ranges from $0.4 \%-1.4 \%$ with no clear dependency on the number of tracks. Only $1 / 3$ of the data was processed with the bug fix, so the total effect in our analysis is $1 / 3$ the size of the effect from the bug. We apply an additional systematic error of $0.5 \%$ to cover the uncertainty due to this effect. 
Table G.1: Comparison of efficiencies with and without overlap bug fix applied in signal MC.

\begin{tabular}{lccc}
\hline \hline \multirow{2}{*}{ Mode } & \multicolumn{2}{c}{ Efficiency $(\%)$} & Ratio \\
without fix & with fix & \\
\hline$\eta_{\eta \pi \pi}^{\prime} K^{+}$ & 23.6 & 23.9 & 0.987 \\
$\eta_{\rho \gamma}^{\prime} K^{+}$ & 29.2 & 29.6 & 0.986 \\
$\eta_{\eta \pi \pi}^{\prime} K^{0}$ & 23.8 & 24.1 & 0.988 \\
$\eta_{\rho \gamma}^{\prime} K^{0}$ & 28.2 & 28.3 & 0.996 \\
$\omega \pi^{+}$ & 21.2 & 21.5 & 0.986 \\
$\omega K^{+}$ & 20.7 & 20.9 & 0.990 \\
$\omega K_{S}^{0}$ & 22.1 & 22.2 & 0.995 \\
\hline \hline
\end{tabular}




\section{Bibliography}

[1] S. Weinberg, Phys. Rev. Lett. 19, 1264 (1967).

[2] Particle Data Group, Y. M. Yao et al., J. Phys. G33, 1 (2006).

[3] Traditional HEP units with $c=1$ are used throughout.

[4] N. Cabibbo, Phys. Rev. Lett. 10, 531 (1963); M. Kobayashi and T. Maskawa, Prog. Theor. Phys. 49, 652 (1973).

[5] M. E. Peskin and D. V. Schroeder, An Introduction to Quantum Field Theory, Westview Press, U.S.A. (1995).

[6] I. I. Bigi and A. I. Sanda, CP Violation, University Press, Cambridge, England (2000).

[7] L. Wolfenstein, Phys. Rev. Lett. 51, 1945 (1983).

[8] J. H. Christenson, J. W. Cronin, V. L. Fitch and R. Turlay, Phys. Rev. Lett. 13, 138 (1964).

[9] The BaBar Collaboration, B. Aubert et al., Phys. Phys. Rev. Lett. 87, 091801 (2001).

[10] P. F. Harrison and H. R. Quinn, editors, The BaBar Physics Book: Physics at an Asymmetric B Factory, Report of the BaBar Physics Workshop, SLAC Report 504 (1998).

[11] The BaBar Collaboration, B. Aubert et al., Phys. Rev. D 70, 012007 (2004).

[12] G. C. Branco, L. Lavoura, and J. P. Silva, CP Violation, Oxford University Press, Oxford (1995).

[13] H. N. Li and S. Mishima, JHEP 0703, 009 (2007).

[14] The BaBar Collaboration, B. Aubert et al., hep-ex/0703021 (2007).

[15] The Belle Collaboration, K. Abe et al., hep-ex/0608039 (2006).

[16] The Heavy Flavor Averaging Group, http://www.slac.stanford.edu/xorg/hfag (2006). 
[17] D. Atwood and A. Soni, Phys. Lett. B405, 150 (1997).

[18] M. Beneke and M. Neubert, Nucl. Phys. B 651, 225 (2003).

[19] Y. Grossman et al., Phys. Rev. D 68, 015004 (2003).

[20] M. Gronau, J. L. Rosner, and J. Zupan, Phys. Lett. B 596, 107 (2004).

[21] M. Gronau, J. L. Rosner, and J. Zupan, Phys. Rev. D 74, 093003 (2006).

[22] M. Beneke, Phys. Lett. B 620, 143 (2005).

[23] A. R. Williamson and J. Zupan, Phys. Rev. D 74, 014003 (2006).

[24] A. Ali, G. Kramer, and C. D. Lü, Phys. Rev. D 58, 094009 (1998).

[25] Y. H. Chen et al., Phys. Rev. D 60, 094014 (1999).

[26] M. Gronau and J. L. Rosner, Phys. Rev. D 53, 2516 (1996).

[27] H. J. Lipkin, Phys. Lett. B 254, 247 (1991).

[28] Except as noted explicitly, we use a particle name to denote either member of a charge conjugate pair.

[29] G. Kramer, W. F. Palmer, and H. Simma, Zeit. Phys. C 66429 (1995).

[30] M. Gronau, Y. Grossman, and J. L. Rosner, Phys. Lett. B 579, 331 (2004).

[31] H. Y. Cheng, C. K. Chua and A. Soni, Phys. Rev. D 72, 014006 (2005).

[32] M. Beneke and M. Neubert, Nucl. Phys. B 675, 333 (2003).

[33] The CLEO Collaboration, S. J. Richichi et al., Phys. Rev. Lett. 85, 520 (2000).

[34] The CLEO Collaboration, C. P. Jessop et al., Phys. Rev. Lett. 85, 2881 (2000).

[35] The CLEO Collaboration, S. Chen et al., Phys. Rev. Lett. 85, 525 (2000).

[36] The BaBar Collaboration, B. Aubert et al., Phys. Rev. Lett. 94, 191802 (2005).

[37] The BaBar Collaboration, B. Aubert et al., Phys. Rev. D 74, 011106 (2006).

[38] The Belle Collaboration, J. Schumann et al., Phys. Rev. Lett. 97, 061802 (2006).

[39] The Belle Collaboration, C. M. Jen et al., hep-ex/0609022 (2006).

[40] The Belle Collaboration, K. F. Chen et al., Phys. Rev. D 72, 012004 (2005).

[41] The BaBar Collaboration, B. Aubert et al., Nucl. Instrum. Methods Phys. Res., Sect. A 479, 1 (2002).

[42] PEP-II Conceptual Design Report, SLAC Report No. SLAC-R-418, (1993).

[43] The GEANT4 Collaboration, S. Agostinelli et al. Nucl. Instrum. Meth. A 506, 250 (2003). 
[44] L. Zhang, http://www.slac.stanford.edu/ zhanglei/RooRarFit/ (2007).

[45] R. Brun et al., http://root.cern.ch/ (2007).

[46] W. Verkerke, D. Kirkby et al., http://roofit.sourceforge.net/ (2007).

[47] ARGUS Collaboration, H. Albrecht et al., Phys. Lett. B 241, 278 (1990).

[48] http://www.slac.stanford.edu/BFROOT/www/Physics/ TrackEfficTaskForce/TrackingTaskForce-2006.html\#Recipes

[49] http://www.slac.stanford.edu/BFROOT/www/Physics/Analysis/ AWG/Neutrals/validation/recipie18.html

[50] http://www.slac.stanford.edu/BFROOT/www/Physics/TrackEfficTaskForce/ TrackingTaskForce-2006.html\#KsRecipe

[51] http://www.slac.stanford.edu/BFROOT/www/Physics/ Tools/Pid/PidOnMc/pidonmc.html

[52] M. Pivk and F. R. Le Diberder, Nucl. Instrum. Methods Phys. Res., Sect. A 555, $356(2005)$.

[53] O. Long, M. Baak, R. N. Cahn, and D. Kirkby, Phys. Rev. D 68, 034010 (2003).

[54] The BaBar Collaboration, B. Aubert et al., Phys. Rev. Lett. 98, 031801 (2007).

[55] The BaBar Collaboration, B. Aubert et al., hep-ex/0607101 (2006).

[56] The BaBar Collaboration, B. Aubert et al., Phys. Rev. D 74, 011106 (2006).

[57] H. Y. Cheng, C. K. Chua and A. Soni, Phys. Rev. D 72, 094003 (2005).

[58] A. R. Williamson and J. Zupan, Phys. Rev. D 74, 014003 (2006). 